\title{
Interconnection of a Forest Growth Model and a Structural Model for Young Poplar Trees (Populus spp.)
}

\author{
Dissertation \\ zur Erlangung des Doktorgrades \\ der Fakultät für Forstwissenschaften und Waldökologie \\ der Georg-August-Universität Göttingen
}

vorgelegt von

Christoph B. Stiehm

geboren in Paderborn

Göttingen, 2019 
1. Gutachter: Prof. Dr. Winfried Kurth

2. Gutachter: Prof. Dr. Jürgen Nagel

Tag der mündlichen Prüfung: 27.09.2019 


\section{Abstract}

When planting fast-growing tree species such as poplars and willows on agricultural land in short rotation coppice plantations, site selection and the associated yield potential pose a central decision for the practitioner. In connection with the cultivar aspect there has been a need for research on the interaction between site and genotype in terms of growth performance. The aim of this work is to examine these questions on several levels. For this purpose, a multi-scale approach was chosen in the framework of which two model complexes are developed which are then connected by an interface.

The first model complex incorporates the implementation of a yield simulator which depicts single tree based growth and mortality as a function of competition and site conditions. The data basis for this is growth data from the joint research project ProLoc funded by the BMEL. For this purpose, 18 trial sites are chosen which were initiated on a broad amplitude of environmental conditions. Following a uniform experimental design, monoclonal trial plots with three poplar and two willow clones (interspecific crossed hybrids) were supervised in two tri-annual rotations and cut back after the third year. Based on the model of the forest growth simulator BWINPro and the associated TreeGross program library, several models are parameterized which, in addition to the survival rates after planting and harvest, estimate the height increment in the first and second rotation. With the distance-independent competition index "basal area of larger trees" the development within the stands can be predicted. Regarding the growth performance on the site level, the parameters of planting date, available water capacity, German agricultural soil quality rating, sum of precipitation in May and June and mean temperature in June and July are identified as influential by variable selection. To estimate the height increment and survival after pruning, tree height before harvest is regarded as an independent variable. The factor clone indicates differences in the growth processes within the models but interactions with site variables can not be determined as significant. Missing variables such as the mean annual increment in dry matter yield in oven-dry tons $h a^{-1} a^{-1}$ are estimated by additional functions parameterized with the dataset. The individual models are connected to a simulation procedure and the overall predictive power is assessed. Good results can be achieved for the first rotation with squared cor- 
relations of the observed and estimated mean stand height of 0.79 . However, in the second rotation the estimation quality decreases to 0.53 . There are single sites with considerable deviations. The depth of the soil sampling and missing extended information on the water supply are suspected as problematic here.

The second model complex includes a structural model focused on the poplar genotypes and the second rotation. First, several measuring methods were identified which are deemed suitable for determining the tree architecture in terms of geometry and topology of the above-ground woody biomass, as well as the morphology of foliage in terms of leaf architecture and leaf shape. For the branch architecture, a manual method and a semi-automatic method with an electromagnetic digitizer for determining branch curvature have been selected and employed. The leaf architecture was measured by a manual method. The leaf shape could be determined by digitizing collected leaves. After analyzing the obtained data, several models are parameterized. As a result, the probability of bud growth and the dimensions and orientation in space of developing shoots can be estimated for apical and lateral buds. The models differentiate between main and minor stems, prolongation and lateral shoots, long and short shoots and, within the lateral shoots, sylleptic and regular shoots. The starting point here is the estimation of the number of internodes per shoot which in turn influences other parameters such as the branch angle and the curvature through the shoot length. Other factors underlying several models are the age, branch order and the genotypic influence. Parameters such as foliage and leaf size can mainly be estimated by the relative height with regard to the absolute tree height. The leaf shape in turn is determined by contour points whose coordinates are calculated as a function of the leaf blade length. As part of the analysis of these models, only slight differences in the structure between the clones are found. Exceptions are the curvature and branching angles of the lateral shoots for one of the clones, for which the models reproduce the observable slender habitus. Significant differences also occur in the leaf shape which reflect the leaf shapes of the underlying parent species of the hybrids. The individual model functions are then implemented into a structural model in the model platform GrolMP. The resulting model can simulate the development of the tree structure for each of the three clones in annual steps. Arbitrarily large stands can be simulated that have realistically varying tree sizes through stochastic components in the model. 
The interconnection of the two model complexes is realized through the import of single tree data from the yield model into the structural model. Two further models are parameterized to determine the number of internodes from the shoot length as annual height increment of the yield model for the structural model and to modify the growth of the minor stems in dependence of the main stem growth. Additionally, the single tree mortality generated by the yield simulator is incorporated into the structural model.

Further research will show whether it is possible to improve the yield model by validation with data from other experiments to include deeper soil layers here. The structural model could be extended to a complete functional structural plant model by incorporating a physiology module. By extending the interconnection to return data from the structural model to the yield model, the predictive power could be improved, for example by means of extended possibilities for modeling the within-stand competition dynamics.

\section{Zusammenfassung}

Beim Anbau von schnellwachsenden Baumarten wie Pappel und Weide auf landwirtschaftlichen Flächen in Kurzumtriebsplantagen stellt die Standortwahl und die daran gebundene Ertragsprognose eine zentrale Entscheidung für den Bewirtschafter dar. In Verbindung mit dem Sortenaspekt besteht hier Forschungsbedarf zur Wechselwirkung von Standort und Genotyp hinsichtlich der Wuchsleistung. Ziel dieser Arbeit ist es, diese Fragestellungen auf mehreren Ebenen zu betrachten. Dazu wurde ein Multiskalen-Ansatz gewählt, in dessen Rahmen zwei Modellkomplexe entwickelt werden, um sie anschließend durch eine Schnittstelle zu verbinden.

Der erste Komplex sieht dabei die Implementierung eines Ertragssimulators vor, der das einzelbaumbasierte Wachstum und die Mortalität in Abhängigkeit von Konkurrenz und Standortbedingungen abbildet. Die Datengrundlage hierfür stellen Zuwachsdaten aus dem vom BMEL geförderten ProLoc Verbundvorhaben dar. Dazu wird auf 18 Versuchsflächen zurückgegriffen, die auf einer breiten Amplitude standörtlicher Eigenschaften angelegt wurden. Nach einem einheitlichem Versuchsdesign wurden monoklonale Versuchsparzellen mit drei Pappelund zwei Weidenklonen (interspezifisch gekreuzte Hybride) in zwei je dreijährigen 
Rotationen versuchstechnisch betreut und nach dem dritten Jahr auf den Stock gesetzt. Basierend auf der Vorlage des Waldwachstumssimulators BWINPro und der zugehörigen Programmbibliothek TreeGross werden mehrere Modelle parametrisiert, die neben den Überlebensraten nach der Pflanzung und dem Rückschnitt die Höhenzuwächse in der ersten und zweiten Rotation schätzen. Mit dem distanzunabhängigen Konkurrenzparameter "basal area of larger trees" kann die Entwicklung innerhalb der Bestände abgebildet werden. Hinsichtlich der Wuchsleistung auf Standortebene stellen sich im Zuge der Variablenselektion die Parameter Pflanzdatum, nutzbare Feldkapazität, Bodenzahl, Niederschlagssumme im Mai und Juni und Mitteltemperatur im Juni und Juli als entscheidend heraus. Zur Schätzung des Höhenzuwachses und der Überlebensrate nach Rückschnitt wird die Baumhöhe vor der Ernte als unabhängige Variable genutzt. Der Faktor Klon deutet innerhalb der Modelle zwar auf Unterschiede in den Wachstumsvorgängen hin, Wechselwirkungen mit Standortvariablen können jedoch nicht festgestellt werden. Fehlende Variablen wie der durchschnittliche Gesamtzuwachs des Ertrags der Trockenmasse in $t_{\text {atro }} h a^{-1} a^{-1}$ werden über zusätzliche am Datensatz parametrisierte Funktionen geschätzt. Die Einzelmodelle werden zu einem Simulationsablauf verbunden und die Gesamtschätzgüte überprüft. In der ersten Rotation können gute Ergebnisse erzielt werden mit quadrierten Korrelationen der beobachteten und geschätzten Bestandesmittelhöhen von 0.79. In der zweiten Rotation nimmt die Schätzgüte jedoch auf $0.53 \mathrm{ab}$. Es finden sich vereinzelte Standorte mit starken Abweichungen, als problematisch werden die Tiefe der Bodenbeprobung und fehlende erweiterte Informationen über den Wasserhaushalt vermutet.

Der zweite Modellkomplex beinhaltet ein Strukturmodell, für das sich auf die Pappel-Genotpyen und die zweite Rotation beschränkt wird. Zunächst wurden mehrere Messmethoden identifiziert, die geeignet sind, die Baumarchitektur in Form von Geometrie und Topologie der oberirdischen holzigen Biomasse sowie die Morphologie der Belaubung hinsichtlich der Blattarchitektur und Blattform zu bestimmen. Für die Verzweigungsarchitektur wurden ein manuelles Verfahren und ein semi-automatisches Verfahren mit einem elektromagnetischen Digitizer zur Bestimmung der Astkrümmung gewählt und angewandt. Die Blattarchitektur wurde mit einem manuellen Verfahren gemessen. Die Blattform konnte per Digitalisierung von eingesammelten Blättern bestimmt werden. Im Zuge der 
Analyse der gewonnenen Daten werden mehrere Modelle parametrisiert. Hierdurch können für Apikal- und Lateralknospen die Austriebswahrscheinlichkeiten sowie die Dimension und Orientierung im Raum von sich bildenden Trieben geschätzt werden. Innerhalb der Modelle wird nach Haupt- und Nebenstämmen, Verlängerungs- und Seitentrieben, Lang- und Kurztrieben und innerhalb der Seitentriebe nach sylleptischen sowie regulären Trieben differenziert. Der Ausgangspunkt ist hier die Schätzung die Internodienanzahl je Trieb, die über die Trieblänge wiederum andere Parameter wie den Verzweigungswinkel und die Krümmung beeinflusst. Weitere Faktoren, die mehreren Modellen zugrunde liegen, sind das Alter und die Verzweigungsordnung sowie der genotypische Einfluss. Parameter wie die Belaubung und die Blattgröße lassen sich mitunter durch die relative Höhe am Baum schätzen. Die Blattform wiederum wird durch Konturpunkte bestimmt, deren Koordinaten in Abhängigkeit von der Blattlänge berechnet werden. Im Rahmen der Analyse dieser Modelle stellen sich geringe Unterschiede in der Struktur zwischen den Klonen heraus. Ausnahmen stellen die Krümmung und Verzweigungswinkel der Seitentriebe für einen der Klone dar, bei dem die Modelle den beobachtbaren schlankeren Habitus gut reproduzieren. Deutliche Unterschiede ergeben sich auch bei den Blattformen, die die Blattformen der zugrundeliegenden Elternspezies der Hybride wiedergeben. Die einzelnen Modellfunktionen werden anschließend als Gesamt-Strukturmodell in der Modellplattform GrolMP implementiert. Das erhaltene Modell kann in Jahresschritten die Entwicklung der Baumstruktur für jeden der drei Klone abbilden. Wahlweise können beliebig große Bestände simuliert werden, die durch stochastische Komponenten im Modell über eine realitätsnahe Variabilität der Baumgrößen verfügen.

Die Verbindung der beiden Modellkomplexe wird durch eine Schnittstelle realisiert, die den Import von Einzelbaumdaten aus dem Ertragsmodell in das Strukturmodell vorsieht. Zwei weitere Modelle werden parametrisiert, um für das Strukturmodell die Internodienanzahl aus der Trieblänge als jährliche Höhenzuwächse des Ertragsmodells ermitteln zu können und das Wachstum der Nebenstämme an den Hauptstamm anzupassen. Darüber hinaus können die vom Ertragssimulator erzeugten Ausfälle in den Beständen berücksichtigt werden.

Zukünftige Forschungsarbeiten werden zeigen, inwiefern das hier entwickelte Ertragsmodell durch eine Validierung mit Daten aus anderen Versuchen weiterentwickelt werden kann, um auch tiefere Bodenschichten mit einzubeziehen. Das 
Strukturmodell könnte durch Einbau eines Physiologiemoduls zu einem vollständigen Funktions-Struktur-Pflanzenmodell ausgebaut werden. Durch die Erweiterung der Schnittstelle zur Rückgabe von Daten vom Strukturmodell zum Ertragsmodell wäre auch eine Verbesserung der Schätzgüte z.B. durch erweiterte Möglichkeiten zur Modellierung der Konkurrenzverhältnisse vorstellbar. 


\section{Acknowledgment}

Several people have supported me in my efforts to finish this thesis and complete all the work that was related to it. In the following paragraphs I would like to express my gratitude to acknowledge their input and support. Unfortunately, I cannot name all people that have helped me during my doctoral studies here therefore I tried to summarize my gratitude for some of the groups that I have been a part of or worked with.

First and foremost, I would like to express my deepest gratitude towards my supervisors. Winfried Kurth enhanced the original idea of implementing a yield model with the aspects of structural modeling and implementing an interconnection. I am very grateful for his continuous and extensive support, the good advice, the possibilities that I was given and for awakening my interest in structural modeling. I would also like to thank Jürgen Nagel for administering the supervision on behalf of the Northwest German Forest Research Institution (NWFVA), his hints for the implementation of the yield simulator and his pragmatic advises. I also have to thank both most warmly for their patience with me finishing my work. I would also like to thank Christian Ammer for his commitment as the third member of the thesis committe.

The NWFVA has been my place of work for the duration of my doctoral studies. I would like to thank both heads of department during that time, Alwin Janßen and Wilfried Steiner, for their trust and providing the necessary freedom in my work for me. Furthermore, I owe great thanks to Martin Hofmann as he initiated the ProLoc project and has been a good and constant advisor with his knowledge on the topic and beyond. I would also like to thank the colleagues from the department of forest genetic resources, working in projects or being part of the permanent staff alike. I profited greatly from the discussions, advice and help that I got over the years and their company made working a lot easier. Beyond the department, I would also like to express my gratitude towards Jan Evers, Thomas Hövelmann and Johannes Sutmöller for their help with the environmental data.

I would also like to thank the German federal ministry of food and agriculture (BMEL) for funding and the agency for renewable resources (FNR) for administering the funds and sponsorship of the ProLoc project. Furthermore I would like to thank the cooperating institutions within the first work package of the ProLoc 
network and especially their executives as well as employees for their contribution and dedication to the project. All contributing personnel can not be named here. Nonetheless, I owe great thanks to everyone involved on the part of the listed institutions:

Manuel Karopka (FVA Baden Württemberg); Klaus Mastel, Frieder Seidl (LTZ Augustenberg); Bettina Stoll, Frank Burger (LWF Bayern); Dirk Freese, Ansgar Quinkenstein (BTU Cottbus); Dieter Murach, Rainer Schlepphorst, Holger Hartmann (HNEE Eberswalde); Dirk Knoche (FIB Finsterwalde); Peter Kaulfuß, Ralf Pecenka (ATB Potsdam Bornim); Lothar Behle Schalk (Universität Giessen); Michael Wachendorf, Reinhold Stülpnagel (Universität Kassel); Ludger Tapken (LK Niedersachsen); Marie-Luise Rottmann-Meyer (3N Niedersachsen); Barbara Boelcke, Andeas Gurgel (LFA Mecklenburg Vorpommern); Norbert Hofnagel (Biomassehof Borlinghausen); Ralf Pude, Volker Wenghöfer, Georg Völkering (Universität Bonn); Henning Kurth (LLFG Sachsen Anhalt); Kerstin Jaekel, Sabine Mau, Pierre Seibold (IfULG Sachsen); Georg von Wühlisch, Stefan Jencsik (Thuenen Institut); Hardy Dembny (Baumschule Oberdorla); Andrea Biertümpfel, Armin Vetter (TLL).

I would also like to thank Christine Wachendorf and Josefine Möller from the soil subproject within ProLoc for their cooperation. All other cooperation partners within the second work package of ProLoc, all former coworkers and colleagues within the cooperation network and the numerous assistants also have my gratitude.

On the part of the university in Göttingen I would like to thank all colleagues at the department of Ecoinformatics, Biometrics and Forest Growth. As it was the case for the colleagues at the NWFVA, I benefited from the discussions and also the pastimes. Especially I would like to thank Michael Henke for very valuable advice on programming in $\mathrm{XL}$ and the provision of the scripts for the leaf shape modeling. Furthermore I would like to thank Katharina Streit for her introduction into using the electro-magnetic digitizer and Peter Surovy for his advice on calibration and other measurement methodology related matters.

Several students have either contributed to the data basis by working as assistants or by writing assignments or degree theses. I would like to thank them for their dedication and the work that they put into it. Foremost, I would like to thank Andrea Plazas Cebrián for the enormous effort that she made for her bach- 
elor thesis which already pointed out important aspects for the further structural modeling.

Apart from work, I would like to thank my friends in Göttingen, at home and beyond for their friendship, support, understanding and help. I would especially like to thank Frederik Hahne for the valuable advice he has given me on programming and related matters that made life easier. I thank Sebastian Schoneberg for the fruitful discussions on statistical matters and work related aspects. I would also like to thank Maximilian Wentzel Vietheer and Philipp Nelis for their help and advice with the final preparations and formal completion of this document.

Finally, I would like to thank my family for their constant support, their help and always being there: my brothers Michael and Sebastian, my sister Claudia, their spouses as well as my nephew and my niece. I express my deepest gratitude to my parents Elisabeth and Burkhard whose permanent support and care enabled me to study forest science and to conclude my doctoral studies in the first place. 


\section{Contents}

1 Introduction 1

1.1 Background . . . . . . . . . . . . . . . . . . 1

1.2 Forest Growth Modeling . . . . . . . . . . . . . . . . . 3

1.2 .1 Different Scales in Forest Growth Modeling . . . . . . . . 3

1.3 Aspects of SRC and Poplar in Forest Growth Modeling . . . . . . 6

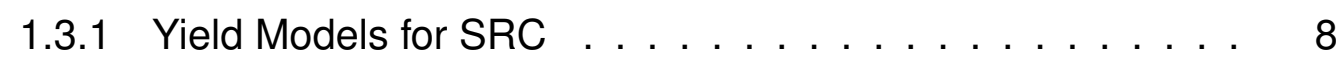

1.3.2 Functional, Structural and Functional-Structural Models for Poplar . . . . . . . . . . . . . . . . . . 14

1.4 Aims $\ldots \ldots \ldots \ldots \ldots \ldots \ldots$

2 Yield Model 21

2.1 Material and Methods . . . . . . . . . . . . . . . 21

2.1 .1 Genotypes . . . . . . . . . . . . . . . . 21

2.1 .2 Trial Sites . . . . . . . . . . . . . . . . . . . . . 22

2.1 .3 Experimental Design . . . . . . . . . . . . . . . 25

2.1 .4 Site Mapping and Climatic Data. . . . . . . . . . . . 31

2.1 .5 Single Tree Based Forest Growth Modeling . . . . . . . 37

2.1 .6 Software and Statistical Analysis . . . . . . . . . . . . 44

2.2 Results . . . . . . . . . . . . . . . . . . 51

2.2.1 Correlation of Environmental Data and Growth . . . . . . 51

2.2 .2 Data Completion with Stand Height Curves . . . . . . . . 55

2.2 .3 First Rotation . . . . . . . . . . . . . . . . . . . . . . . 57

2.2 .4 Second Rotation . . . . . . . . . . . . . . . . . . 73

2.2 .5 Overall Simulator Performance . . . . . . . . . . . . . 86

2.2 .6 Simulator Implementation . . . . . . . . . . . . . . 90 
$\begin{array}{lll}3 & \text { Structural Model } & 96\end{array}$

3.1 Material and Methods . . . . . . . . . . . . . . . . . . . 96

3.1.1 Methods for Measuring Tree Structure . . . . . . . . . . 96

3.1 .2 Trial Sites . . . . . . . . . . . . . . . . . . . . . . . . 101

3.1 .3 Tree Architecture . . . . . . . . . . . . . . . . . . 102

3.1 .4 Branch Curvature . . . . . . . . . . . . . . . . 110

3.1 .5 Leaf Position and Structure . . . . . . . . . . . . 120

3.1 .6 Leaf Shape . . . . . . . . . . . . . . . . . . . . . 126

3.1 .7 Statistical Modeling and Model Procedure . . . . . . . . 131

3.1 .8 Software . . . . . . . . . . . . . . . . . . . . . 132

3.1.9 Interconnection of Yield Model and Structural Model . . . . 133

3.2 Results . . . . . . . . . . . . . . . . . . . . . . . . 135

3.2 .1 Stool Architecture . . . . . . . . . . . . . . . . . . . 135

3.2 .2 Shoot Growth . . . . . . . . . . . . . . . . . . . . . . . . 139

3.2 .3 Count of Internodes per Growth Unit . . . . . . . . . . 147

3.2.4 Length of Growth Units and Internodes . . . . . . . . . 160

3.2 .5 Branch Orientation and Directional Angle . . . . . . . . . 164

3.2 .6 Diameter of Growth Units . . . . . . . . . . . . . . . . 168

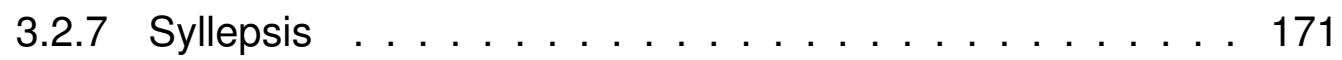

3.2 .8 Branch Curvature . . . . . . . . . . . . . . . . . . . . 175

3.2 .9 Leaf Position and Structure . . . . . . . . . . . . . 183

3.2 .10 Leaf Shape . . . . . . . . . . . . . . . . . . . . . . 205

3.2 .11 GrolMP Implementation . . . . . . . . . . . . . . . . 214

4 Interconnection 225

4.1 Statistical models . . . . . . . . . . . . . . . . . . . . . . 225

4.2 Implementation . . . . . . . . . . . . . . . . . . 228

5 Discussion 232

6 Appendix 257

Bibliography 


\section{Glossary}

Listed here are only abbreviations that are used extensively and are not always accompanied by a direct explanation in the text or in the Figure and Table captions.

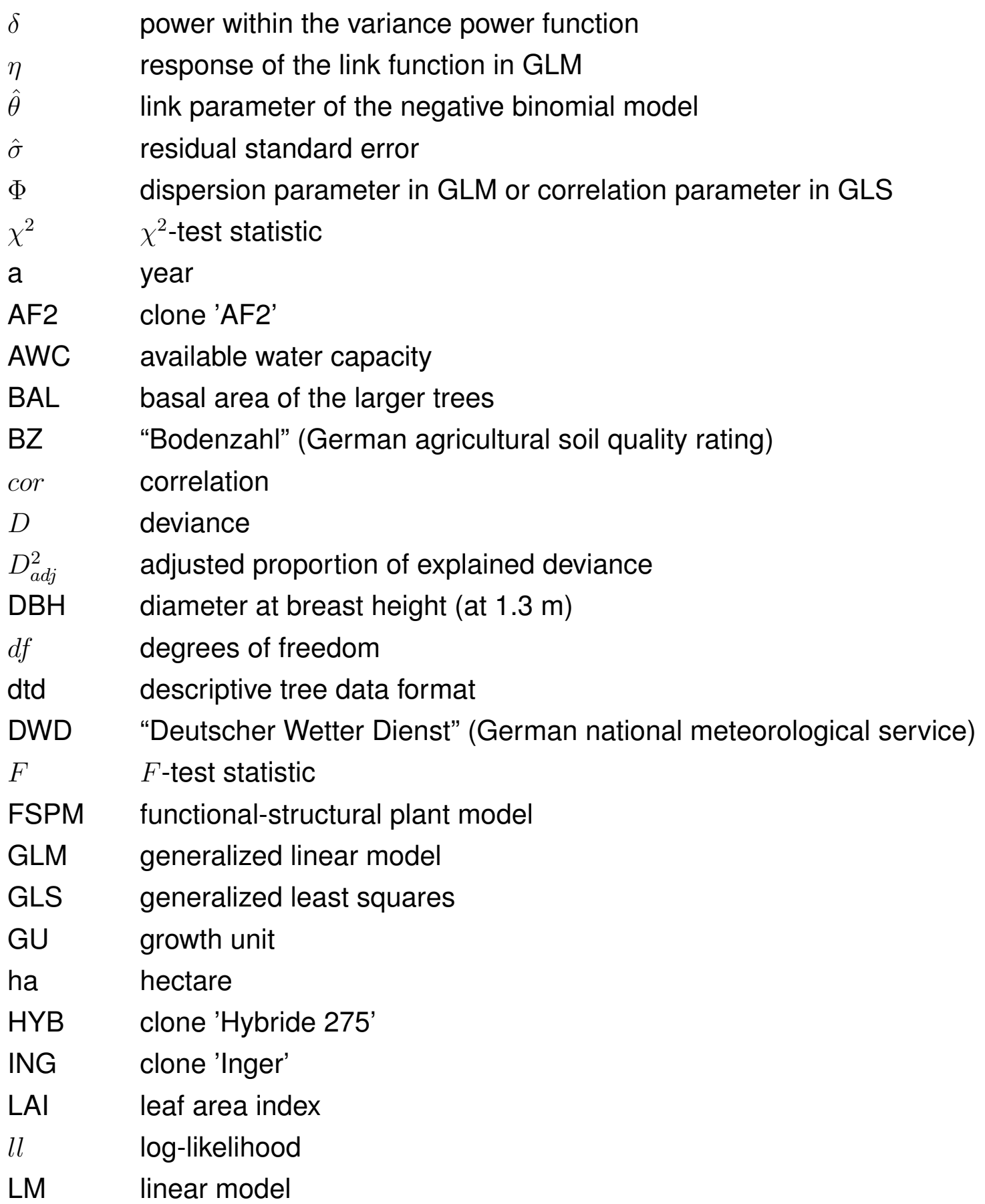


LRT likelihood ratio test statistic

$M \quad$ arithmetic mean

MAX clone 'Max 1'

Max maximum

MAI mean annual increment

$M d n \quad$ median

Min minimum

MTG multi-scale tree graphs

$n$ number of observations, sample size

odt oven-dry ton

$p \quad p$-value (probability value)

$Q \quad$ quantile

$r \quad$ correlation coefficient

$R_{a d j}^{2} \quad$ adjusted coefficient of determination

RCD root collar diameter (at $0.1 \mathrm{~m}$ )

$R S S \quad$ residual sum of squares

$s \quad$ standard deviation

se standard error

SRC short rotation coppice

$t \quad t$-test statistic

TOR clone 'Tordis'

$z \quad z$-test statistic 


\section{Introduction}

\subsection{Background}

The cultivation of poplars and willows in short rotation coppice (short: SRC) plantations on agricultural acreage has been assessed as a promising option in providing biomass as a renewable energy source especially in regard to low carbon abatement costs (Wissenschaftlicher Beirat Agrarpolitik beim Bundesministerium für Ernährung, Landwirtschaft und Verbraucherschutz (WBA), 2007). Against the background of the fast and vigorous growth of these tree species, especially in comparison to species found in classical Central European forestry (Bärwolff et al., 2012), the interest in their utilization has been subject to changing phases of rise and fall. Research on the cultivation of trees belonging to the genera Salicaceae, which includes the aforementioned species, reach back to the time following the second world war. Especially for poplar, a peak in interest and research efforts was reached in this period due to the low lumber supply as a result of the reparation payments (Fell, 1992). The main application here was planting fast growing tree species in forests and the landscape as well as the recultivation of former mining sites. After a following decline (Fell, 1992), some research efforts, starting in the late 1970s in part due to the oil crisis (Knust, 2009), shifted the focus of poplar and willow cultivation towards the possible utilization of these tree species in densely spaced plantations on agricultural land for producing biomass as an energy source or for the pulp and paper industry (Hofmann, 2005). Research activities continued and peaked again with the rise in demand for alternative and renewable energy sources due to the energy system transition and nuclear energy phaseout in Germany. Over the course of the described research climaxes several projects focused on a considerable variety of aspects from forest and agricultural sciences which themselves incorporated biological, economical and ecological questions. Along with resolving challenges regard- 
ing the technical implementation of the plantations, breeding new high yielding cultivars and estimating the growth potential of this cultivation form based on environmental conditions were at the forefront of research efforts. Within this framework, amongst others, two joint research projects (FastWOOD and ProLoc) were funded by the German Federal Ministry of Food and Agriculture through the Agency for Renewable Resources to tackle questions regarding the aspects of breeding (project FastWOOD) and yield estimation (project ProLoc). The motivation for FastWOOD originated in the fact that breeding efforts, while being a long-term task, had not been continuously made but were being taken up again, especially in foreign countries, during the 1990s (Liesebach and Schneck, 2018). Although poplars and willows are well suited for breeding, due to their genetic properties and possibilities in hybridization and clonal propagation amongst others (Liesebach and Schneck, 2018), mainly cultivars from older programs were available for practitioners. FastWOOD aimed at closing this gap while gaining further knowledge on fast-growing tree species (Janßen et al., 2010). Besides the supply of newly bred high yielding clones through FastWOOD, some questions still remained regarding the yield potential of SRC in interaction with environmental conditions (soil and climate). Existing research was in many cases limited to single clones or a small range of environmental conditions and offered also limited possibilities in making statements on the yield determining site factors that stakeholders need to take into consideration when planning a plantation (Janßen et al., 2017). Combining and comparing the results of existing research was further hindered by different experimental methodologies or changed management options like rotation cycle or spacing. The joint research project ProLoc was hence initialized to answer the question on genotype-environment interaction in yield potential by using a fixed set of poplar and willow clones on a broader range of soil and climate conditions in an uniform experimental design. ProLoc was initiated in 2008 and ended in 2015 after two funding phases and a short hiatus in between. The first phase was coordinated by the Hessian competence center for renewable resources ("HessenRohstoffe"). Coordination in the second phase was continued by the Department of Forest Genetic Resources at the Northwest German Forest Research Institute ("Nordwestdeutsche Forstliche Versuchsanstalt", short: NW-FVA). A network of (at the end of the project) 21 cooperation partners from the public and private sector collaborated to establish over 30 trial sites with an 
uniform trial design using 3 poplar and 2 willow cultivars. More details on the experimental methodology will be given Section 3.1. As already stated, the main goal was to assess the general yield potential of SRC plantations based on environmental conditions and identify if there are any interactions with the genotypic factor. These questions and the data that was acquired to answer them were the basis for this work. The main goal is to examine growth in SRC plantations by employing tools and approaches from forest growth modeling on multiple scales and finding possibilities for connecting these scales.

\subsection{Forest Growth Modeling}

\subsubsection{Different Scales in Forest Growth Modeling}

Following Kurth (1994b), plant models can be differentiated by different approaches into 3 main categories (see Figure 1.1).

\section{aggregated \\ models}

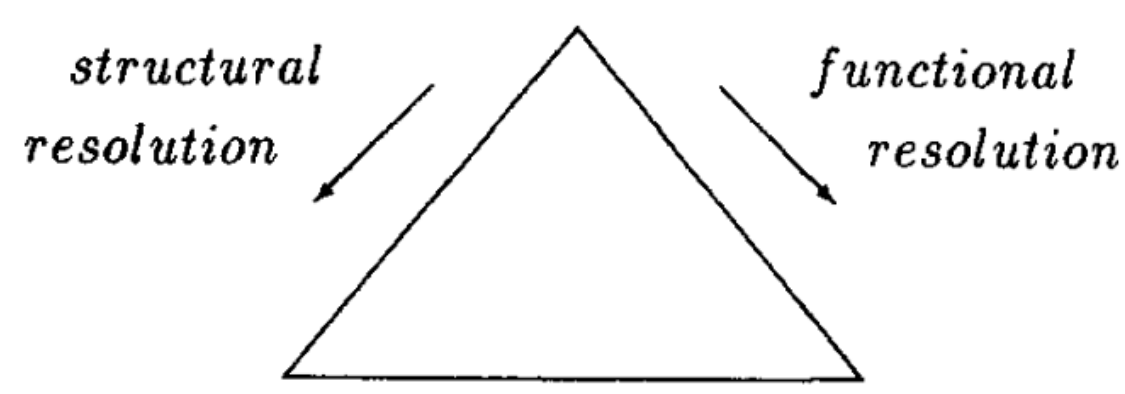

morphological models process

models

Figure 1.1: Triangle schema of different types of plant models (Figure from Kurth, 1994b, p. 300).

Regarding scale and resolution, aggregated models have a comparably coarse approach. Concerning trees, this category is most prominently represented by stand models in forestry (Kurth, 1994b). Starting with yield tables the complexity 
and sophistication of these model types has increased in the last century with the development of distribution models towards the implementation of single tree based growth models (Pretzsch, 2019). Due to the fast development of computational power, the resolution of empirically based stand models has therefore increased from the population to the organism level (Pretzsch, 2019). Several single tree based growth simulators have been implemented like SILVA (Pretzsch et al., 2002), SIBYLA (Fabrika, 2005, cited in Fabrika and Pretzsch (2013)) or BWINPro (Nagel et al., 2002). A central motivation for furthering this approach were the shortcomings of simple stand level models like yield tables in estimating growth especially within mixed species forest stands with more complex stand dynamics (Pretzsch, 2019).

Coming from the organism scale, the resolution of the modeling approach can be further elaborated towards more detailed and finer organizational levels within the tree. Following the categorization by Kurth (1994b) this increase can either focus on the morphology of a tree and its components (morphological/structural models) or the functional processes and their relationships (process/functional models). Morphological models are focused on the arrangement, size and shape of a tree's components (woody biomass like roots, stems and branches as well as foliage for example) in 3D space (referred to as geometry (Danjon and Reubens, 2008)) and the structural relations of these components (topology (Danjon and Reubens, 2008)). Important groundwork from a botanical viewpoint has been provided by Hallé et al. (1978) and their development of an architectural analysis of trees. Process models are more concerned with physiological questions relating for example to biomass production through photosynthesis, water uptake and balance, transpiration, nutrient balance and cycling within the plant and in interaction with the environment that surrounds the plant.

Arranging models into these categories is not mutually exclusive and no hard borders can be drawn here as models exist that combine the inherent methodologies (Kurth, 1994b). Pretzsch (2019) lists for example hybrid models that combine properties of empirical stand growth models with functions that approximate the physiology of biomass production based on environmental parameters.

An approach that combines the properties and fundamentals of morphological and process models are Functional-Structural-Plant-Models (short: FSPMs) (Buck-Sorlin, 2013a). This is an integrated concept that considers the mutual de- 
pendency of morphology and physiology in plants (Buck-Sorlin, 2013a). Figure 1.2 depicts the composition of an FSPM and how the different modules regarding structure and function interact.

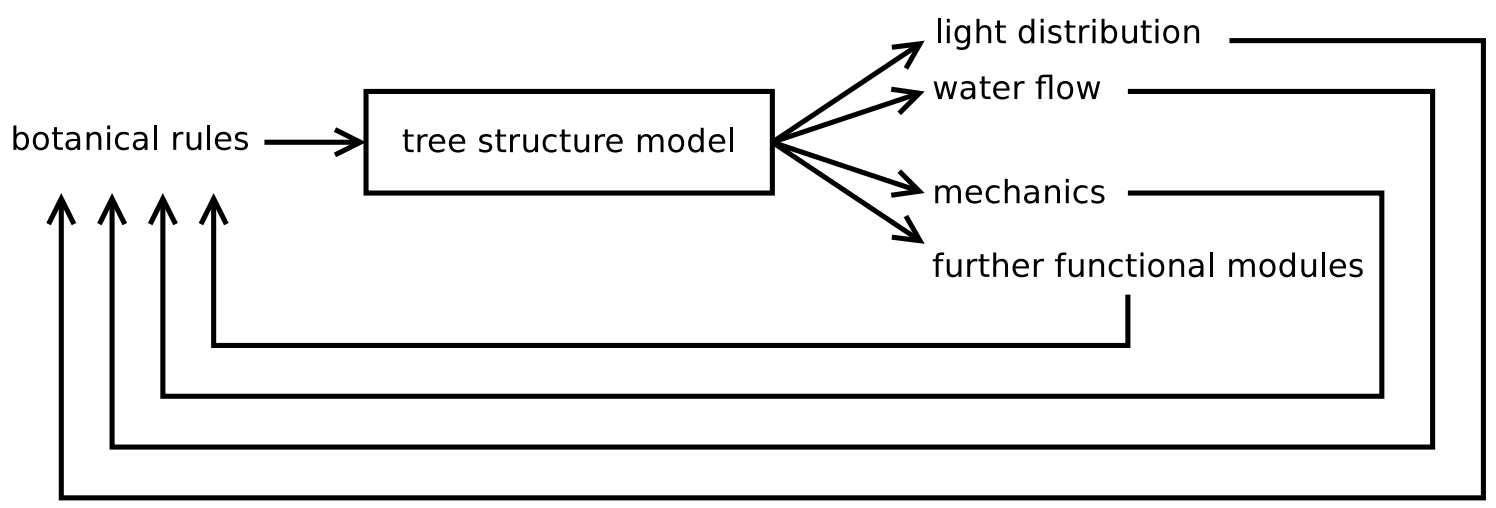

Figure 1.2: Basic design and principles of an FSPM (Figure adapted from Figure 3 in Kurth and Anzola Jürgenson, 1997, p. 19).

The FSPM paradigm has seen a rise in application and growing interest in the last years (Vos et al., 2010; Sievänen et al., 2014). Regarding the theoretical formalisms of how to simulate the development of plants, important groundwork has been made by the establishment of Lindenmayer-systems (short: L-systems). These were formed by the botanist Aristid Lindenmayer who first used them to describe the development of algae (Lindenmayer, 1968). L-systems are a rulebased approach in which the latter refers to string rewriting (Buck-Sorlin, 2013b). Through a set of rules, which are being applied in parallel, symbols are being replaced by strings (Buck-Sorlin, 2013b). The resulting string can be graphically interpreted for visualization (Buck-Sorlin, 2013b). A more extensive overview on the subject can be found in Prusinkiewicz and Lindenmayer (2004, originally published in 1990) which also introduces some extensions of L-systems like stochastic components. The theoretical framework has subsequently been expanded by graph theory (Buck-Sorlin, 2013b) through the introduction of growth grammars (Kurth and Sloboda, 1997; Kurth, 1994a) and later on relational growth grammars (Kurth et al., 2005). These developments were accompanied by the implementation of software, modeling platforms, their associated programming/specification languages and syntax. For the interpretation of growth grammars the software tool GROGRA (Kurth, 1994a) was implemented. For relational growth grammars which extended growth grammars by aspects from object-oriented modeling 
and programming (Kurth et al., 2005), the programming language $\mathrm{XL}$ and with it the modeling platform GrolMP were implemented (Kniemeyer, 2008). Several other software solutions for functional-structural plant modeling exist currently like OpenAlea (Pradal et al., 2008). For an overview see Sievänen et al. (2014).

The question on which model to employ depends primarily on the research question that needs to be answered. Aggregated models are usually preferred for a coarser scale and can be helpful as a support tool in decision making due to their, in comparison, simplicity and easier comprehensibility. Additionally, their implementation has been preferred due to lack of knowledge on the more detailed, underlying processes and the lower demand in resources (like measurement effort or computational power) that the more sophisticated modeling of these processes demand. Trivially, the success and growing utilization of models focusing on more detailed scales like single tree based growth models or FSPMs is consequentially, in part, owed to the rapid development in computer technology and the growing knowledge on plants, including their internal processes and interaction with their surroundings, based on findings made in plant biology and, in this special case, forest science.

The aim of this work is to demonstrate the possibilities in parameterizing a single tree based model and a structural model, both with an applied background in SRC plantations, as well as the connection of both approaches. The following Section will give an overview on the properties of SRC for modeling along with prior work on the subject matter. Finally the concrete aims will be defined which then open for the description of the data acquisition, analysis and model implementation.

\subsection{Aspects of SRC and Poplar in Forest Growth Modeling}

Before focusing on existing work that has already addressed the analysis of growth dynamics in SRC plantations some of the properties of this cultivation system should be described. Under Central European conditions SRC plantations are usually established with fast growing tree species on agricultural land (Bärwolff et al., 2012). The mainly used tree species are poplars and willows 
due to their vigorous growth (Bärwolff et al., 2012). The plantations are usually planted in a fixed rectangular spacing (row design) with high planting densities (Bärwolff et al., 2012). The latter depends on the production aim as the produced biomass can be used as a resource for energy supply, in the form of woodchips for example, or as material for the pulp and paper industry, in the form of trunk wood (Bärwolff et al., 2012). For establishing SRC stands, cuttings or rods are used as planting material (Bärwolff et al., 2012). Site preparation, weed regulation and planting is usually realized with a high degree of mechanization (Bärwolff et al., 2012). Due to the possibility of vegetative propagation of poplars and willows, high yielding clones can be employed. The plantations are often monoclonal without any form of mixture (Knust, 2009) . Based on the production aim, woodchips or trunk wood, the plantations are harvested in rotation cycles of varying length (Bärwolff et al., 2012) of up to 20 years (Landgraf et al., 2018) which are, however, short in comparison to classical forestry cultivation in Central Europe. Based on the production aim, the spacing is chosen in combination with the rotation length. Landgraf et al. (2018) distinguish 3 types of management options here:

- Minirotation: rotation length 2-5 years, $7000-16000$ trees $h a^{-1}$, almost exclusively for production of wood chips for bioenergy

- Midirotation: rotation length 6-12 years, 3000 - 6000 trees $h a^{-1}$, production of wood chips or trunkwood

- Maxirotation: rotation length $12-20$ years, $<3000$ trees $h a^{-1}$, production of trunkwood

This additionally implies that these pure stands are even aged. Based on the planting density, SRC plantations can be harvested, usually during the winter period, with adapted agricultural machinery or forestry harvest machinery (Bärwolff et al., 2012). This means that a clear cut regime is being applied to the stand by harvesting the above-ground biomass while leaving the root stock in the ground. Poplars and willows, depending on the genotype, have a high ability for resprouting which takes place within the vegetation period after cutting. This usually leads to a more vigorous growth due to the beneficial relation of root stock size and developing shoots (Bärwolff et al., 2012). It also leads to a change in morphology as the number of shoots per stool increases by resprouting (Bärwolff et al., 2012). 
As already pointed out, the factor genotype plays an important role in SRC cultivation. The choice of clonal material plays an important role in planning and execution. Due to their advantageous properties for hybridization, poplars and willows were subject of several breeding programs aiming for higher yield and properties that support this aim like pest resistance (Knust, 2009). As it is closely linked with yield potential some research efforts were concentrated on finding yield determining structural properties which could, for example, optimize light interception. This mounted to the idea of the formulation of an ideotype in breeding (Ceulemans, 1990; Koski and Dickmann, 1992; Dickmann et al., 2002) which itself can be developed through structural analysis and linking this to genetics (Wu, 1998). Of course the physiology aspect is also closely linked to this and it has been suggested to further focus on photosynthetic efficiency when selecting clones (Bartels, 1987; Dickmann et al., 2002).

Apart from breeding towards optimized growth properties, especially poplars have been subjected to interest in research efforts as a model organism due to their fast growth and genetic properties (Bradshaw et al., 2000; Taylor, 2002; Cronk, 2005).

As it could be shown, SRC cultivation has properties that constitute considerable differences to classical forestry and that, complemented by the possibilities that the utilized tree species present, have given rise to several questions that have already been addressed by extensive research in this field. Based on this, an overview on existing work on different levels of aggregation will be given in the following section. This is separated into yield modeling (primarily on the stand level) as well as functional, structural and functional-structural plant modeling.

\subsubsection{Yield Models for SRC}

This section gives an outline on previous publications in the field of yield modeling for SRC plantations. The presented publications have been chosen as examples for different methodological approaches or relevance to the data that was used in this thesis. The selection here is by no means exhaustive and the results of other research efforts will be taken up in the following chapters. 


\section{Top-Down Growth Model by Corona et al. (2002)}

Corona et al. (2002) have devised an approach called "disaggregation" that, in terms of the introduced model triangle, corresponds to moving away from the upper tip of aggregated models. This incorporates the estimation of annual increment of the stand-level basal area in dependence of age, site index and the stand-level basal area at the beginning of the time step. Based on the standlevel growth, the growth in diameter classes is estimated which itself is then used to estimate single tree growth. The first two steps produced satisfactory results with coefficients of determination $R^{2}$ above 0.5 . The 4 plantation stands were not thinned or coppiced. Measurements took place in 3 year intervals repeatedly from stand ages of 5 to 16 years. The clone 'I-214' which is quite common in Italian poplar cultivation was planted. For refining the estimation of single tree growth, a competition index was calculated comprising the directly neighboring trees in the plantation. This did not improve the modeling results and helped little in explaining the remaining variance. Corona et al. (2002) point out though that the competition could be a negligible factor in the examined plantations due to the comparably wide spacing of $5 \times 5 \mathrm{~m}$.

\section{Potential Analysis by Aylott et al. (2008)}

Based on trial plot data, Aylott et al. (2008) made an effort to upscale the results with geo-information data on agricultural land for assessing the potential of SRC cultivation in England and Wales. Different scenarios in upscaling were differently focused on set-aside land. The trial plots were supervised over the course of 2 rotations each with a rotation length of 3 year. Within the trial series 16 genotypes were used: 8 for Populus and 8 for Salix, although not the full set of clones was planted on all sites. On a wide range of environmental properties 49 sites were established. The spacing was realized in a double row system, as an optimization for harvesting technology, with $0.75 \mathrm{~m}$ and $1.5 \mathrm{~m}$ between row distances and $0.75 \mathrm{~m}$ and $1.5 \mathrm{~m}$ and $0.9 \mathrm{~m}$ within row distances. The environmental variables that were used for modeling were available water, soil acidity, soil texture, a geological indicator, topography and climate in the form of precipitation and temperature in different aggregations. Different models were fit for each clone and the variables with the highest influence on yield were selected. The models were 
then used to assign predictions to raster data of agricultural land for assessing the yield potential. The predicted mean annual increment ranged from 5 to 11 oven-dry tons per hectare per year (odt $h a^{-1} a^{-1}$ ). The willows performed better than the poplar clones although Aylott et al. (2008) point out that comparably old clones were used here. The overall predictive power of the models is satisfactory with correlations of observed and estimated values above 0.5. Based on these results, Aylott et al. (2008) predict a high potential for SRC in the energy supply of the United Kingdom.

\section{Boundary Line Yield Model by Murach et al. (2008)}

Murach et al. (2008) have used the boundary-line method which focuses on the maximum potential influence an environmental variable can have on a growth parameter. By using the latter as a response, grouping them according to an environmental, independent variable and taking only each group's maximum values into consideration, a regression is implemented. The variance within the groups is attributed to other limiting factors per site. The independent variable that was used by Murach et al. (2008) is the available transpiration water which was calculated based on the available water capacity within a soil depth of $50 \mathrm{~cm}$, the sum of precipitation during the vegetation period, the interception and capillary rise from the groundwater table. Data from stands with differing stand ages and plant densities was utilized. The spacings ranged from 111111 to 222222 trees $h a^{-1}$ for poplar and from 8888 to 14815 trees $h a^{-1}$ for willow. The different ages were standardized for comparison. The standardization differed for poplar and willow, additionally the rotation lengths were not uniform ( 3 years for willow, 5 years for poplar). 10 willow genotypes and 11 poplar genotypes were used. The latter could produce mean heights from 3 to $10 \mathrm{~m}$, the former reached heights between 2 and $6 \mathrm{~m}$. The range of the mean annual increment however was comparable for both genera with 5 to 16 odt $h a^{-1} a^{-1}$. Based on the regression analysis of the boundary line method a positive influence of the available transpiration water can be established. Due to differentiation between the available transpiration water groups a further positive influence of the capillary rise is assumed. The mean height is estimated based solely on the available transpiration water, for the mean annual increment the spacing is added. Higher spacings tended to produce higher yields for both poplar and willow genotypes. Concerning the clone choice, 
Murach et al. (2008) refrain from a recommendation on which genera to prefer as there is no clear differentiation in yield. The comparably small data basis of the yield modeling is critically reflected upon. An extension of the approach by including more trial sites is presented in Murach et al. (2009) which points towards higher yield potentials for poplar although it is noticed that the environmental factor differed also with the factor clone and that the utilized willow clones do not include newly bred cultivars. The model is finally used for a potential analysis of SRC cultivation in Brandenburg by Murach et al. (2008) which is extended to Saxony by Murach et al. (2009).

\section{Yield Potential Model by Ali (2009)}

Also as a basis for a yield potential analysis of SRC in the German federal state of Saxony, Ali (2009) has implemented a modeling procedure for estimating growth of poplar plantations based on environmental variables. The foundation for the modeling process was data from 6 trial sites in Saxony with stand ages from 1 to 9 years. The modeling procedure was separated into 2 steps which were further split for 4 clonal groups that were assigned based on parentage of the clones and an assessment of their growth dynamics. First, the top height per stand was modeled depending on stand age and environmental variables. Using multiple linear regression the variables that were selected were the available water capacity, the German agricultural soil quality rating, mean temperature from April to July and sum of precipitation from May to June. The second modeling step was used for modeling the biomass yield based on the obtained top height and stand density. Additional data was integrated into the fitting procedure of the second step. Very high coefficients of determination of $R^{2}>0.97$ were achieved with this procedure. Ali (2009) points out that there is still room for improvement by expanding the model to willow clones and adding a broader range of environmental conditions to the data basis. The model was implemented into a computer program that was made available (currently not the case anymore) on the web page of the forest growth department of the technical university in Dresden. 
Yield Model for Plantation Initiation by Bergante et al. (2010)

Bergante et al. (2010) have identified variables influencing growth and survival in SRC plantations through the methodology of stepwise regression analysis. The data basis were yield and survival values from 11 different trials at 6 sites in northern and central Italy. The stand density of the plantations varied from 5747 to 10000 trees $h a^{-1}$. Site preparation and planting was oriented according to common practice. Some sites were irrigated and fertilizer was applied during the first rotation. The trials included 183 plots with 17 poplar clones and 102 plots with 4 willow clones. The data was acquired during the first rotation cycle with a rotation length of 2 years. Environmental variables were measured per site and comprised soil analysis and climate data from weather stations in the proximity. Soil analysis was carried out to a depth of 25 to $30 \mathrm{~cm}$. Acquired parameters were soil texture, soil acidity, nitrogen and organic carbon content. The climatic variables that were considered were mean temperature and precipitation in different aggregations. Together with a dummy variable of fertilized and non-fertilized plots and the planting density, the environmental variables were used as an input for the model selection via stepwise regression analysis. The model parameterization was separated into 3 groups for the poplar (2 separate groups) and willow clones ( 1 separate group). The mean annual yield ranged from 7 to 8.8 odt $h a^{-1} a^{-1}$ for the three groups. The models that were chosen produced a satisfactory to good predictive power with adjusted coefficients of determination $R_{a d j}^{2}$ ranging from 0.54 to 0.83 . For modeling the yield within the 3 groups exclusively variables that contain the amount of precipitation and irrigation were identified as having a significant influence. For the survival rates, in 2 groups no variables could be identified as having a significant influence and in 1 group clay content and precipitation were selected. Bergante et al. (2010) therefore indicate precipitation and irrigation as the central parameters influencing growth and survival.

\section{Yield Model by Amthauer Gallardo (2014) Based on ProLoc Data}

Mainly based on the data from the first ProLoc project phase and hence the first rotation, Amthauer Gallardo (2014) implemented several models for estimating the mean annual increment. The data basis is widely the same as for the first rotation used in this thesis. 20 sites were selected while others were left out due 
to not being representative (problems with weeds regulation e.g.) or being special cases in terms of soil conditions (moors, post mining sites).

While parameters like mean stand height, mean diameter at breast height, survival rate were examined, the influence of environmental variables on mean annual increment was the focal point of the analysis. For the latter, the trial locations were separated into 3 clusters based on their soil texture. The separated clusters were sand, silt and loam and a general cluster containing all sites. Modeling the mean annual increment was then realized within the 3 soil texture clusters and the general cluster. Not all genotypes were used in every cluster. The independent variables that were used were climate parameters in different monthly aggregation. The parameters mainly comprised precipitation, temperature and aridity index as a combination of the two former variables. Concerning the soil, parameters like the German agricultural soil quality rating and soil physics parameters like available water capacity, soil texture or dry bulk density were tested. Furthermore, the climatic and soil variables were further processed by calculating their products or ratios for acquiring aggregated soil/climate variables. Higher yields were produced on loess soils with higher silt content while this is contrasted by poor yields on sandy soils with lower available water capacity. In the general cluster, the available water capacity had the most significant influence on growth. For temperature, a negative correlation with mean annual increment could be proved in some cases while for precipitation the opposite applies. Regarding the temporal aggregation, mostly earlier time periods during the vegetation period produced better results. Using aggregated/combined variables of soil and climate data and separately fitting them within the soil texture clusters improved the predictive power of the models. In general, no major differences between clones or genera regarding an interaction with the environmental variables could be identified although in some cases the models were still fit separately. Additionally to the yield modeling, allometric biomass functions for the clones were fit to estimate dry mass based on diameter at breast height. Furthermore, based on the approach by Horn et al. (2013) and data from Biertümpfel et al. (2009, 2012), a forward projection of yield potential is made for following rotation cycles. Amthauer Gallardo (2014) points out that the projection should be consolidated integrating more data into the modeling procedure. 


\subsubsection{Functional, Structural and Functional-Structural Models for Poplar}

As proven, poplar has some favorable properties for modeling. In this section some of the plant models (structural, functional or FSPMs) that have already been implemented with poplar as a model organism are presented. As it was the case for the yield models, this serves rather as an overview and is not exhaustive.

\section{ECOPHYS Model by Rauscher et al. (1990)}

Rauscher et al. (1990) elaborate on the advantages of ecophysiological growth process models of single trees versus deterministic, empirical forest growth models. They implemented the ECOPHYS model as the former model type for simulating the growth of juvenile poplar trees of the clone 'Eugenei' $(P$. deltoides $\times P$. nigra) during the establishment year. The single leaves are the central unit of this model. The foundations of it are that growth is influenced by single leaves, that the photosynthetic rate is mainly determined by microenvironmental factors (solar radiation and temperature) on the leaf level and that these factors are itself conditioned by leaf orientation (Rauscher et al., 1990). Furthermore the photosynthates are allocated among the sinks meristem and respiration while this allocation is controlled by the microenvironment and genotype (Rauscher et al., 1990). The factors of water and nutrient availability are assumed to be non limiting. The model can be utilized for other clones by specifying a set of input variables like date of bud break or ratio of leaf blade width to length. The time step of the model is 1 hour although photosynthate production can be summarized in longer time spans. Besides the photosynthate production, the allocation of these within the plant, the conversion of photosynthates to biomass, the estimation of physical dimensions based on biomass and the respiration are simulated (Rauscher et al. 1990). The functions for these estimations were parameterized with data from several experiments on different scales. The model was validated by comparing simulation output with experimental data which appears overall satisfactory but increasingly differs for some parameters with an advanced point in time of the vegetation period. The authors discuss the assumptions of non limiting resources (water and nutrients) and that stem growth is simulated in more detail than root growth. Possible use-cases for the model are as a selection aid in breeding pro- 
grams, for evaluating the influence of adverse environmental conditions on plant growth or as a basis for incorporating new findings into ecophysiology (Rauscher et al., 1990).

\section{3-D CPCA Structural Model by Casella and Sinoquet (2003)}

Casella and Sinoquet (2003) emphasize the differences in poplar genotypes regarding their structure of woody biomass and leaves and how these factors influence the growth performance. Following Godin et al. (1999) the authors have gathered data on 2 clones with differing yield: 'Ghoy' ( $P$. deltoides $\times P$. nigra, described as low yielding) and 'Trichobel' ( $P$. trichocarpa described as higher yielding). The trees were cut after the first year and then harvested in 3 year rotation cycles. The spacing was designed as double rows with between-row distances of $0.75 \mathrm{~m}$ and $1.5 \mathrm{~m}$ and within-row distances of $0.9 \mathrm{~m}$. The measuring took place in the second year of the second rotation cycle (above-ground biomass aged 1-2 years and below-ground biomass aged 1-6 years) repeatedly during the vegetation period. Structural data (topology and geometry) was acquired for woody and leaf biomass. Amongst other parameters, the shoot orientation was quantified by elevation and azimuth/divergence angle, length of internodes and curvature for $20 \mathrm{~cm}$ segments was measured. Leaf morphology was approximated by measuring orientation of petiole and leaf blade as well as their size. Sylleptic shoots from the first year of the rotation cycle were not considered. Regression models were parameterized for some of the relationships to enable a simulation of structure. The components/organs within the structural model were represented by conic frustums (e.g. internodes), cylinders (e.g. stool and petiole) and flat surfaces (e.g. leaf blade). The simulated structures were visualized with the POV-Ray ray-tracing software (Version 3.5, available via povray.org). The obtained visualizations were used for generating virtual hemispherical photographs which were then compared with actual hemispherical photographs from the plantation. The comparison was made visually and by comparing the canopy openness from the simulated photographs. The overall model performance was assessed as good although some deviations remain depending on the point in time during the vegetation period and the clone. The authors list some possible applications of their model like being utilized as module for other studies if simulating a canopy is needed. 


\section{Expansion of Process Model SECRETS by Deckmyn et al. (2004)}

As an answer to the limitations of empirical growth models in short rotation forestry and the difficulties when extrapolating estimations to new poplar clones or previously untested environmental conditions, Deckmyn et al. (2004) expanded the process model SECRETS by Sampson and Ceulemans (1999). The SECRETS model itself is composed of several modules consisting of other models for e.g. photosynthesis, carbon storage and partitioning or respiration (see full list in Deckmyn et al., 2004). The time step of the original model was adapted which produced estimations for daily values of respiration, transpiration, photosynthesis and all carbon stocks. Climate data was simulated based on real data from another experiment and two types of soil (differentiated by their texture as loam and sand) were used. Some adaptions to the model functions were made to account for the properties of poplar SRC cultivation. Some basic management variables like spacing $\left(10,000 n h a^{-1}\right)$ were set. Other management variables like irrigation, fertilization and rotation length were varied in the modeling process. Their influence on the yield of the plantation was quantified and simulation results were compared with measured data of two different clones 'Hoogvorst' ( $P$. trichocarpa $\times P$. nigra) and 'Fritzi Pauley' (P. trichocarpa). By assessment of the authors, growth and yield are being estimated considerably well by the model. The model underestimates the leaf area index in the first year after coppicing. The authors recommend further validation of the model under different environmental conditions (soil, climate) and with more clones over longer time periods.

\section{Poplar FSPM by Buck-Sorlin et al. (2005)}

The FSPM for juvenile poplar by Buck-Sorlin et al. (2005) has an exemplary character for demonstrating the possibilities and advantages of RGG and XL. In parts, the model is based on ECOPHYS. The length of the time steps is 1 hour. Input parameters are the daily progress of solar radiation in the form of photosynthetically active radiation and the temperature. In combination with the leaf area of each simulated leaf this is the basis for calculating the production of photosynthates. This is further influenced by whether the leaf is shaded or not. The simulated tree develops from a seed which has its own photosynthate reserves for initializing growth. Growth incorporates the development of new leaves and internodes by a 
meristem in defined time intervals (plastochron) and the extension of already existing organs depending on the available photosynthates. Growth and transport processes are separated into alternating steps due to technical reasons. Each leaf has 4 senescence stages which affect the photosynthesis function. Another feature of the model is a metabolic regulatory network representing the synthesis of gibberellic acid and two of its metabolic precursors. With a modification of this network the results of an experiment with transgenic poplar and a modified morphology could be reproduced with the simulated plants. The model can be obtained from the authors. A modified version is available on the grogra website (grogra.de).

\section{GreenLab Adaption by Gang et al. (2011)}

Gang et al. (2011) have implemented an FSPM for poplar by expanding the GreenLab model (Yan, 2004). 4 trees of Populus $\times$ euramericana clone 'Neva' (also named clone 107) of ages 3 to 6 were cut and a representative sample of branches was measured. This included recording mass, length, diameter and the number of nodes per GU (by counting the leaf scars). Additionally, leaves were grouped into 3 classes by size and on 3 leaves per class the surface and fresh mass were acquired. The measured trees were from a plantation with a $4 \times 3$ spacing and had not been harvested before therefore they have a singlestem morphology. Gang et al. (2011) discuss that the simulated trees represent the topology of the probed clone quite well. Parameters and processes like the insertion height of lateral shoots, branch curvature, secondary growth and development of below-ground biomass have not been measured. These aspects challenge additional measurements and will be tackled in future work since the presented model is based on a preliminary study (Gang et al., 2011).

\section{Expansion of the 3-PG Process-Based Model by Headlee et al. (2013)}

As listed in the following paragraph, 3 publications have adapted the 3-PG model for estimating poplar yield and 1 publication for willow yield. The focus is put here on the most recent publication.

Following Amichev et al. (2010) and Amichev et al. (2011), Headlee et al. (2013) expanded the Physiological Processes Predicting Growth model (developed by 
Landsberg and Waring (1997) and adapted by Sands (2004a,b) for probable cultivation of 3 P. deltoides $\times$ P. nigra clones ('DN17', 'DN34' and 'DN182') in the northern US states of Wisconsin and Minnesota. The motivation was to be able to estimate the yield of poplar plantations outside of the then current range of cultivated sites. The modeling procedure of the 3-PG framework is to estimate the maximum potential yield by light radiation, temperature and photosynthesis functions that have a species-specific parameterization. From the maximum yield potential the actual growth is estimated by introducing limiting factors like site quality and water supply (Headlee et al., 2013). By allometric functions the photosynthates are allocated to the different tree components. For the hybrid poplar parameterization of this procedure several sources (literature and data) were sighted and processed. Instead of a clone specific parameterization, mean values were taken. The overall performance of the model was assessed as good with an $R^{2}=0.89$ for a linear regression between observed and predicted values. Deviations between the two are partially higher for some sites. The model was then used for spatial modeling of growth potential in the two federal US states. The authors mention potential for improvement regarding the more detailed incorporation and validation of development of underground biomass. Further clones and their inherent physiological attributes as well as additional site conditions should be included. Also, the authors recommend to rather use the model for predictions on a regional scale. Local scale estimations should be made with further refined site values. The latter has been tackled by Zalesny et al. (2012).

\section{Structural Model by Plazas Cebrian (2014)}

For a bachelor thesis, Plazas Cebrian (2014) implemented a structural model with XL in GrolMP based on structural data acquired from the 3 poplar clones in the ProLoc project on a ProLoc site. The data and the findings have been incorporated into the modeling procedure of this work and will be described in more detail later. The data was measured with the dtd format (also described later). This allowed for a 3D representation of the measured trees. Via regression, some functions were parameterized to describe the architectural relationships. By testing, no significant differences between clones were found in structure, hence only a single model was parameterized for all 3 clones. The model already approximates the coppiced tree's structure quite well, mainly depending on size relations 
and the covariates shoot age and order. Still lacking are leaf morphology, branch curvature and a physiological component of the model.

\subsection{Aims}

Based on the already existing research and its connected models as well as the data that was already available the following aims were defined for this thesis.

- Yield model complex

- Based on the ProLoc data find a suitable, parsimonious approach to simulate stand growth on a single tree level.

- Parameterize the models needed for the simulator and identify the yield determining variables. The latter refer to environmental, genotypic and competition parameters as well as their possible interactions.

- Implement the growth simulator based on the existing simulator BWINPro and the program library TreeGrOSS.

- Structural model complex

- Select and employ a suitable methodology for measuring structure of above-ground woody biomass and leaves.

- Devise a modeling framework to represent the relationship of these measured parameters. Parameterize the models to describe said relationships.

- Implement the parameterized models in GrolMP using XL.

- Interconnection

- Implement a one-directional interconnection between the yield model and the structural model by passing increment data from the yield model to the structural model.

The first part of this endeavor included both the poplar and willow genotypes from ProLoc, while the structural model complex and the interconnection solely focus on the poplar genotypes. In the following chapters the materials and methods as well as results will be separately presented for the yield model and then 
for the structural model combined with the interconnection. The discussion will then be combined again for all aspects. 


\section{Yield Model}

\subsection{Material and Methods}

In the following Section on the experimental design of the ProLoc project some of the passages are a summary or reproduction of the methodology description, which is in German, from the final project report (Janßen et al., 2017) and a publication from within the project (Stiehm and Hofmann, 2018). In both cases the descriptions were written by the author of this thesis.

\subsubsection{Genotypes}

The genotypes chosen for testing include three poplar and two willow hybrid clones. Table 2.1 contains an overview of all clones, their species and section. The motivation for selecting these clones was to utilize cultivars that were already well established in practical cultivation in Germany (for poplar see Anonymous (2016) and Anonymous (2018)). Additionally these hybrids represent different parentages which were expected to show different reactions or plasticity to site conditions. 
Table 2.1: Overview of the utilized clones within ProLoc, meta data and parentage information. Species and sex information for 'Max 1' taken from Anonymous (2012), for 'Hybride 275' and 'AF2' taken from Niemczyk et al. (2016), for 'Inger' and 'Tordis' taken from Caslin et al. (2012). Section information was derived from Dickmann and Kuzovkina (2014) based on species affiliation.

\begin{tabular}{|c|c|c|c|c|}
\hline Clone Name & Species & Section & Sex & Code \\
\hline 'Max 1' & $\begin{array}{l}\text { P. nigra Linnaeus } \times \\
\text { P. maximowiczii Henry }\end{array}$ & $\begin{array}{l}\text { Aigeiros } \times \\
\text { Tacamahaca }\end{array}$ & q & MAX \\
\hline $\begin{array}{l}\text { 'Hybride 275' } \\
\text { (syn. 'NE 42') }\end{array}$ & $\begin{array}{l}\text { P. maximowiczii Henry } \times \\
\text { P. trichocarpa Torrey \& Gray }\end{array}$ & $\begin{array}{c}\text { Tacamahaca } \times \\
\text { Tacamahaca }\end{array}$ & $0^{7}$ & HYB \\
\hline 'AF2' & $\begin{array}{l}\text { P. deltoides Marshall } \times \\
\text { P. nigra Linnaeus }\end{array}$ & $\begin{array}{l}\text { Aigeiros } \times \\
\text { Aigeiros }\end{array}$ & $0^{7}$ & $\mathrm{AF} 2$ \\
\hline 'Inger' & $\begin{array}{r}\text { S. triandra Linnaeus } \times \\
\text { S. viminalis Linnaeus }\end{array}$ & $\begin{array}{l}\text { Amygdalinae Koch } \times \\
\text { Vimen Dumortier }\end{array}$ & q & ING \\
\hline 'Tordis' & $\begin{array}{l}\text { (S. schwerinii E. Wolf } \times \\
\text { S. viminalis Linnaeus) } \times \\
\text { S. viminalis Linnaeus }\end{array}$ & $\begin{array}{l}\text { (Vimen Dumortier } \times \\
\text { Vimen Dumortier) } \times \\
\text { Vimen Dumortier }\end{array}$ & o & TOR \\
\hline
\end{tabular}

\subsubsection{Trial Sites}

Initially, 38 sites were planted at the beginning of the project. Some of these sites were dropped out of the trial series due to management problems and/or too poor survival rates and growth (Amthauer Gallardo, 2014). Some of the trial sites were discontinued in the second project phase due to administrative reasons (Janßen et al., 2017). This amounted to 12 sites that were not actively supervised through the course of both rotations. All trial sites are listed in Table 2.2 together with their supervising institution and their status at the end of the second rotation. The geographic location of the sites is presented in Figure 2.1. Without the discontinued sites, 26 trials remained that were available for data analysis. In accordance with Amthauer Gallardo (2014) some further stands were dropped from the modeling process due to weed pressure, vole browsing, trials that were established on post mining sites and moors. For some sites single clones were dropped due to quality problems with the planting material which led to poor survival rates and growth (Amthauer Gallardo, 2014). The site and clone combinations that were used for the yield modeling are listed in Table 2.3 . 
Table 2.2: Overview of the ProLoc trial sites. Their location identifier (Loc ID), the location name, the institution that supervised them and whether the trial site was still actively maintained by the end of the second rotation are given.

\begin{tabular}{|c|c|c|c|}
\hline Loc ID & Location name & Supervising institution & Status \\
\hline pl01 & Emmendingen & FVA Baden-Württemberg & active \\
\hline pl02 & Liliental & FVA Baden-Württemberg & active \\
\hline pl03 & Aulendorf & LTZ Augustenberg & active \\
\hline pl04 & Forchheim & LTZ Augustenberg & active \\
\hline pl05 & Ladenburg & LTZ Augustenberg & discontinued \\
\hline pl06 & Kupferzell & LTZ Augustenberg & active \\
\hline pl07 & Kaisheim & LWF Bayern & active \\
\hline pl08 & Energiewald Welzow & BTU Cottbus & active \\
\hline pl09 & Löwenberg & BTU Cottbus & discontinued \\
\hline pl10 & Neuruppin & BTU Cottbus & discontinued \\
\hline pl11 & Cahnsdorf & HNE Eberswalde (FH) & discontinued \\
\hline pl12 & Kummerow & HNE Eberswalde (FH) & active \\
\hline pl13 & Grünewalde & FIB Finsterwalde & active \\
\hline pl14 & Welzow Süd & FIB Finsterwalde & active \\
\hline pl15 & Potsdam Bornim & ATB Potsdam & active \\
\hline pl16 & Lehmkaute & JLU Gießen & active \\
\hline pl17 & Unterrieden & Universität Kassel & active \\
\hline pl18 & Gülzow & LFA Mecklenburg-Vorpommern & active \\
\hline pl19 & Werlte & 3N Kompetenzzentrum Niedersachsen & active \\
\hline pl20 & Borlinghausen & MR Höxter-Warburg & active \\
\hline pl21 & Dollendorf & LWK Nordrhein-Westfalen & discontinued \\
\hline pl22 & Haus Düsse & LWK Nordrhein-Westfalen & discontinued \\
\hline pl23 & Wildbergerhütte & ZebiO - Zentrum für Bioenergie & discontinued \\
\hline pl24 & Königshovener Höhe & RWE / RFWU Bonn & active \\
\hline pl25 & Campus Klein Altendorf & RFWU Bonn & active \\
\hline pl26 & Am Kandel & FH Trier & discontinued \\
\hline pl27 & Bärenrode & LLFG Sachsen-Anhalt & active \\
\hline pl28 & Bernburg & LLFG Sachsen-Anhalt & active \\
\hline pl29 & Hayn & LLFG Sachsen-Anhalt & active \\
\hline pl30 & Iden & LLFG Sachsen-Anhalt & active \\
\hline pl31 & Pommritz & LFULG Sachsen & active \\
\hline pl32 & Thammenhain I & TU Dresden & discontinued \\
\hline pl33 & Thammenhain II & TU Dresden & discontinued \\
\hline pl34 & Zschadrass & TU Dresden & discontinued \\
\hline pl35 & Trenthorst & TI - Fachinstitut für Forstgenetik Großhansdorf & active \\
\hline pl36 & Lohberg & Baumschulen Oberdorla GmbH & active \\
\hline pl37 & Dornburg & Thüringer Landesanstalt für Landwirtschaft & active \\
\hline pl38 & Heiliges Marpingen & FH Trier & discontinued \\
\hline
\end{tabular}


Table 2.3: Overview of the site clone combinations that were used for the yield modeling procedure. Cells of combinations containing "input" were used, "dropped" indicates omitted combinations.

\begin{tabular}{llllll}
\hline Loc ID & MAX & HYB & AF2 & ING & TOR \\
\hline pl01 & input & input & input & input & input \\
pl04 & input & input & input & input & input \\
pl06 & input & dropped & input & input & dropped \\
pl12 & input & input & input & input & input \\
pl15 & input & input & input & input & input \\
pl16 & input & dropped & input & input & input \\
pl17 & input & input & input & input & input \\
pl18 & input & input & input & input & input \\
pl19 & input & dropped & input & input & input \\
pl20 & input & input & input & input & input \\
pl25 & input & input & input & input & input \\
pl28 & input & input & input & input & input \\
pl29 & input & input & input & input & input \\
pl30 & input & input & input & input & input \\
pl31 & input & input & input & input & input \\
pl35 & input & input & input & input & input \\
pl36 & input & dropped & input & input & input \\
pl37 & input & dropped & input & input & input \\
\hline
\end{tabular}




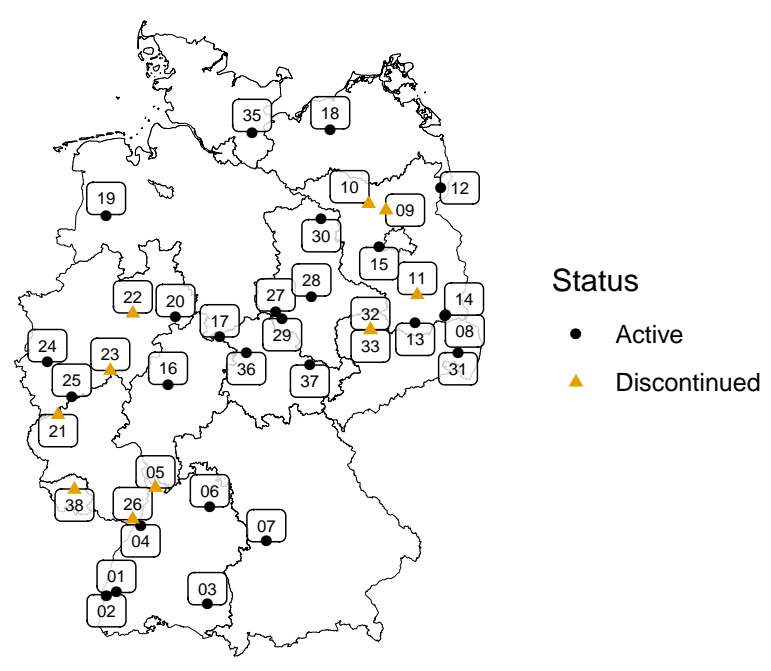

Figure 2.1: Map of all initiated trial locations in ProLoc separated by whether they were still active at the end of the second rotation or discontinued. The trials are marked by their location ID (the leading "pl" was left out here for a clearer arrangement). State borders by Hijmans et al. (2015)).

\subsubsection{Experimental Design}

According to standards in agricultural and forest tree breeding (Williams et al., 2002; Thomas, 2006) the experimental design has been laid out as a multilocation trial series. The design of a single trial site has been laid out as fully randomised with 4 replications per clone resulting in 20 plots per site. On some sites the design fulfills the requirements of a randomised complete block design. Since this has not been the case for all sites the trials were considered as fully randomised within the analysis. Figure 2.2 displays an exemplary trial design (here for pl17 Unterrieden). Not all sites were laid out rectangularly as shown here. 


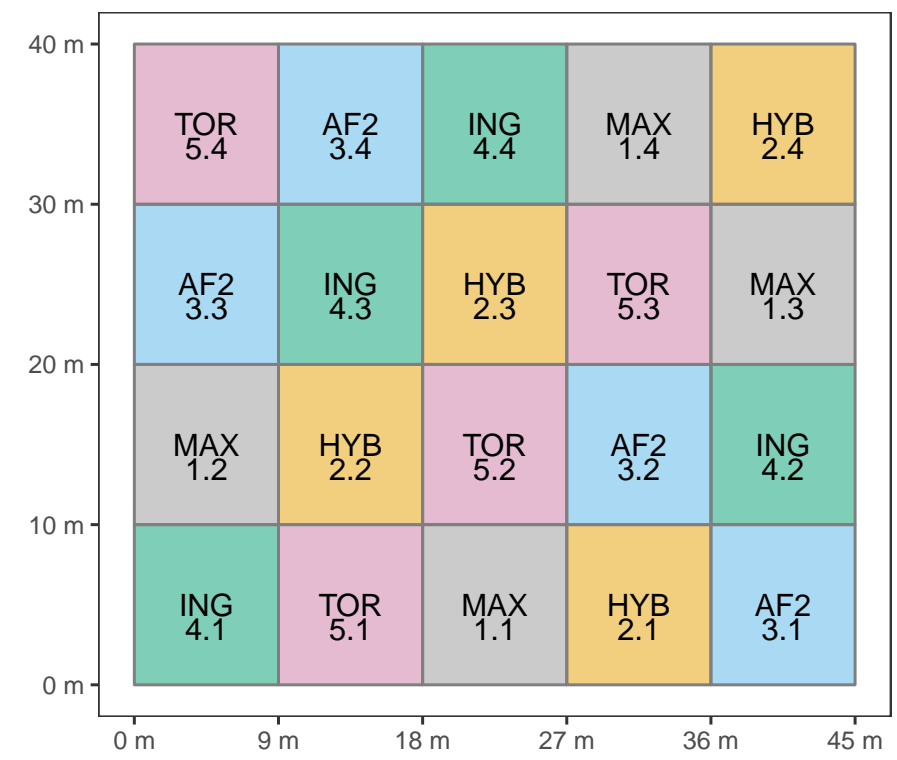

Figure 2.2: Example for the experimental design and plot layout in a single field trial (pl17 Unterrieden). The different plots are marked by the abbreviation of the clone they contain and an identifier that stands for the clone (first digit) and the replication (last digit).

The spacing for the whole trial series has been fixed to a within row distance of $0.5 \mathrm{~m}$ and a between row distance of $1.8 \mathrm{~m}$, resulting in a density of 11111 individuals $h a^{-1}$. The overall size of each trial added up to $1800 \mathrm{~m}^{2}$. Additionally each site should have a surrounding $5 \mathrm{~m}$ wide margin to neglect possible unwanted border effects by the surrounding environment. Planting rows were aligned between successive plots for establishing the connected areal structure of a plantation within a single trial. Additionally a main working direction for measuring was facilitated. Ideally measurements were carried out in the direction from south to north within rows and from west to east between rows. Deviations from this procedure occurred due to the terrain exposition on some sites. Each plot consists of five rows with 20 trees per row. An example for the single plot experimental design is given in Figure 2.3 . 


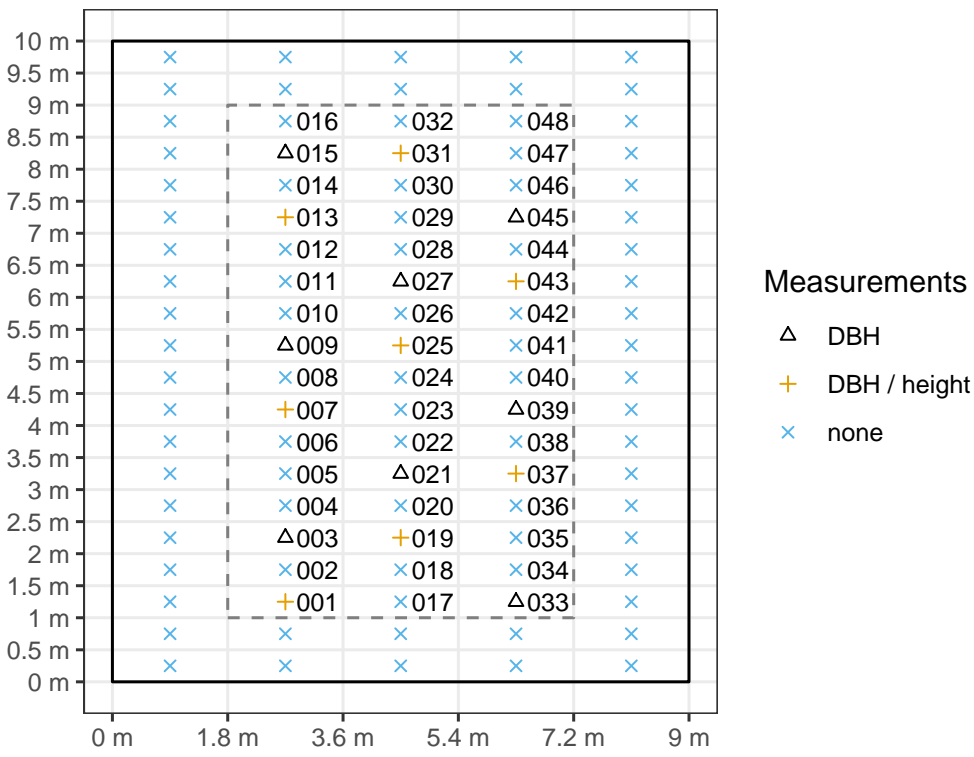

Figure 2.3: Example for the experimental design and tree numbering in a single trial plot. The solid black line marks the plot border, the gray dashed line marks the plot's core in which the measurements were conducted. The gray grid lines mark the individual growing space of each tree determined by the spacing. All 100 trees are represented by a shape and can be distinguished by shape and color into trees that were not measured, trees whose DBH was measured and trees whose height was measured additionally to the $\mathrm{DBH}$. The measurement grid is displayed here as it was defined in the second project phase. The respective tree numbers are shown right next to the shapes. Shown is a plot from the first repetition, the trees in other plots are numbered consecutively in the same manner (049 to 192).

Per plot a margin containing the whole first and fifth row as well as the first and last two trees of the second, third and fourth row has not been measured to neglect possible border effects between plots. Measurements were conducted in the core of each plot containing the remaining 48 individuals. A fixed sampling design in the form of a grid beginning at the first plant was applied to each plot's core. Two sample sizes were defined. One dense grid containing every second tree $(n=24)$ and a less dense grid containing every sixth tree $(n=8)$. These different intensities were chosen for the measurement of different variables (see below). Due to the increased number of shoots after resprouting the dense grid was reduced to a number of $n=16$ trees per plot. Previously measured trees were kept in the grid for comparability. Within the first project phase 
missing plants were skipped in incremental measuring. The next available plant was used instead even though this might have deviated from the fixed sampling design. This procedure was dismissed at the first harvest after 3 years, further incremental measurements then were homogenized to a consistent adherence of the sampling grid. This was factored in for the calculation of areal plot values like basal area per hectare.

\section{Planting / Trial Establishing}

The preparations at each trial site were arranged individually due to different prior land use on each field. The overall aim was to ensure homogeneous growth conditions. The measures for this ranged from physical seed bed preparation to chemical weeds regulation. Detailed information on the site preparation can be obtained from Amthauer Gallardo (2014). Planting was carried out manually by using $20 \mathrm{~cm}$ cuttings. The period during which the trials that are used here for modeling were planted ranged from the 24th of April to the 19th of May 2008. Replanting of losses in 2009 was done between the 9th of March and the 11th of April. An overview of the planting dates is given in Table 2.4. The trials, including the surrounding margin, were fenced in after planting to prevent browsing damage by game (except for pl16 and pl36). 
Table 2.4: Overview of the planting dates. The first column contains the location identifier. The second column contains the actual date of planting and the third column contains the ordinal date as day of year of planting. Both dates refer to the point in time where planting began. For location pl16 no data was available therefore the mean value of the ordinal data was taken.

\begin{tabular}{llr}
\hline Loc ID & Date of planting & $\begin{array}{c}\text { Ordinal date (day of year) } \\
\text { of planting }\end{array}$ \\
\hline pl01 & $2008-05-07$ & 128 \\
pl04 & $2008-04-24$ & 115 \\
pl06 & $2008-05-15$ & 136 \\
pl12 & $2008-04-30$ & 121 \\
pl15 & $2008-04-28$ & 119 \\
pl16 & $2008-05-04$ & 125 \\
pl17 & $2008-04-24$ & 115 \\
pl18 & $2008-04-26$ & 117 \\
pl19 & $2008-04-30$ & 121 \\
pl20 & $2008-05-09$ & 130 \\
pl25 & $2008-04-24$ & 115 \\
pl28 & $2008-05-06$ & 127 \\
pl29 & $2008-05-13$ & 134 \\
pl30 & $2008-05-07$ & 128 \\
pl31 & $2008-04-29$ & 120 \\
pl35 & $2008-04-29$ & 120 \\
pl36 & $2008-05-19$ & 140 \\
pl37 & $2008-04-28$ & 119 \\
\hline
\end{tabular}

\section{Measuring}

Several variables were measured during ProLoc for assessing the yield and yield influencing parameters per clone. A clarification needs to be made first. Due to the harvest after 3 years, the morphology or habitus of the cultivated poplar and willow clones changes. Before cutting, fewer stems grow from the planted cuttings and after coppicing a higher number of new stems emerges from the stool that is left in the ground (Sennerby-Forsse et al., 1992). This was also the case for the ProLoc clones (Janßen et al., 2017). In contrast to classical forest cultivation, a single tree can have multiple stems. For each tree the growth parameters of all stems were measured, except for the mass weighing which will 
be described later. Annually during winter, growth parameters were measured. This included the breast height diameter (short: $\mathrm{DBH}$ ) at $1.3 \mathrm{~m}$ per stem, using the dense sampling grid. During the first rotation cycle additionally the root collar diameter (short: RCD) at a stem height of $0.1 \mathrm{~m}$ was measured. The shoot length was measured using the sparse grid of 8 trees per plot. The procedure of measuring the incremental variables was regulated in compliance to standards in forest inventory (see Kramer and Akça, 2008).

The degree of areal coverage by weeds and infestation with pathogens were measured annually during the summer period. This data was not further considered here because only sites that were on an uncritical level were selected for data analysis.

During the course of both harvests, biomass yield was estimated using the sampling tree method described by Röhle (2009). Described here is the weighing methodology from the second harvest which mainly differed by the number of trees measured from the first harvest. A documentation for the latter is presented by Amthauer Gallardo (2014).

The trial sites were harvested manually. Only stems from trees within the dense DBH measuring grid were considered. Each tree was cut at a stump height of $0.1 \mathrm{~m}$. All stems from a single tree were weighed together as a whole individual. Trees were weighed either raw or as woodchips for determining the whole tree fresh biomass to a reading accuracy of $100 \mathrm{~g}$ steps. Following this procedure the dry matter content per clone and trial site was quantified by randomly choosing 4 vital trees from each plot. Per tree, a mixed sample of $2 \mathrm{~kg}$ fresh wood was taken. If the total mass of an individual tree was below $2 \mathrm{~kg}$, the whole tree was processed for the mixed sample. If the trees were weighed as already processed woodchips, the sample was taken from the woodchips. If the trees were weighed as a whole, each individual tree was segmented into three equally large parts, taking $20 \mathrm{~cm}$ length out of each segment's mid as a cross section containing stems and branches. The material was split lengthwise and additionally crosswise depending on its diameter: 3 to $5 \mathrm{~cm}$ diameter split once, above $5 \mathrm{~cm}$ split twice. The material was then collated again for the sample per tree. Each sample was weighed still in the field to a reading accuracy of $1 \mathrm{~g}$. Samples were then dried at $103^{\circ} \mathrm{C}$ in drying chambers to constant mass based on regulations by the German DIN norm (DIN Deutsches Institut für Normung e.V., 2010). The dry mass was 
then weighed and the dry matter content calculated from the ratio of dry mass to fresh mass. The dry matter content was averaged per harvest, trial site and clone. Fresh mass per tree was then corrected with this value to dry mass. Dry masses of trees were summed up per plot. Based on the number of trees within the measuring grid this sum was then projected to the area of 1 hectare. This produced the total biomass per rotation cycle based on the associated harvest. Division by the length of each rotation cycle, 3 years, results in the mean annual increment (short: MAI). The latter is the usual value in Germany for comparing yield potential of SRC plantations (Röhle, 2013). The formula for MAI estimation is (modified from Amthauer Gallardo (2014)):

$$
M A I\left[\text { odt } h a^{-1} a^{-1}\right]=\frac{\sum_{i=1}^{n} \text { dry mass single tree } e_{i}[\text { odt }] * \text { planting density }\left[N \mathrm{ha}^{-1}\right]}{\text { sample size plot core }[n] * \text { stand age }[a]}
$$

The remaining 84 individuals per plot were also cut at a height of $0.1 \mathrm{~m}$. The extant biomass was harvested and completely transported off the trial site.

\subsubsection{Site Mapping and Climatic Data}

\section{Soil}

Site mapping was divided into the surveying and assessment of physical and chemical parameters. This included the digging of soil profiles and their full analysis as well as soil probing at each trial site during the first project phase. A full documentation of the probing can be obtained from Amthauer Gallardo (2014). Here the focus is set on summarizing the methodology and concentrating on the variables that were used in the growth modeling process. For the methodology of the ProLoc site surveying the widely used standard of the German soil mapping guideline "KA5" (Ad-Hoc-Arbeitsgruppe Boden der Staatlichen Geologischen Dienste und der Bundesanstalt für Geowissenschaften und Rohstoffe and Sponagel, 2005) was adopted. For soil physics, parameters like the dry bulk density, stone content and soil texture were determined by probing and laboratory analysis. A key variable that combines these physical properties is the available water capacity (short: AWC). This variable has been previously shown to be important for estimating yield in short rotation coppice (Ali, 2009) which was also the 
case for stand level modeling in ProLoc (Amthauer Gallardo, 2014; Janßen et al., 2017). There are different methods available for calculating the AWC. The "KA5" provides a widely accepted standard for calculating the AWC based on soil texture, dry bulk density and organic matter content. Renger et al. (2009) suggested improvements to the calculation basis, evaluating it as imprecise in some cases. Amthauer Gallardo (2014) used the methodology of Renger et al. (2009) for the ProLoc data as well. The AWC values have been recalculated for yield modeling here because there were inconsistencies in the data from the first project phase.

Another soil parameter that has proven useful in modeling SRC yield is the German agricultural soil quality rating ("Bodenzahl", short: BZ). The BZ serves as an orientation for the yield potential of a site in growing agricultural crops (Blume et al., 2010). The BZ is scaled as a positive integer ranging up to 100 points which implies optimal conditions for crop cultivation in Germany (Blume et al., 2010). The calculation of the BZ relies mainly on the soil texture and geological age and status class of the examined soil. By adding the factors climate and slope to the assessment the "Ackerzahl" can be derived from the "Bodenzahl" (Blume et al., 2010). Ali (2009) and Amthauer Gallardo (2014) have both used the "Ackerzahl" as a soil quality index in modeling. Here it was decided to use the BZ instead, since climate was entered separately into the models and slope was mostly not present or negligible for the sites tested here.

As already indicated by the organic matter content, soil chemistry was also probed. This included nutrients like nitrogen (along with $\mathrm{C} / \mathrm{N}$ ratio), phosphorus and potassium and other parameters like $\mathrm{pH}$ as a measure for soil acidity. Both variables regarding soil physics and chemistry were tested for their influence on growth. In testing and selecting these variables it was however decided soon that the soil chemistry was dropped from the modeling procedure because either its influence was negligible or implausible. This decision will be discussed later.

Regarding the factor groundwater, which is often discussed as important, (Wittich, 1951; Duhme, 1989), the assessment of ground water level done in ProLoc is rather unreliable since it was based on measurement of water levels in nearby wells (Amthauer Gallardo, 2014). It was dropped from the modeling process as an independent variable because it led to rather implausible results.

A disputable aspect is though that the probing was only conducted for soil depths 0 to $30 \mathrm{~cm}$ and 30 to $60 \mathrm{~cm}$ which is common in agriculture but might 
cut short for the rooting depth of poplars and willows. Within a subproject which focused on carbon dynamics (Heyn and Wachendorf, 2012) the soil depth 60 to $90 \mathrm{~cm}$ was also measured. This was done on 20 sites, of which 11 fall into the subset that was chosen for modeling here. This data was not further used for modeling here to keep the environmental data homogeneous.

Table 2.5 contains an overview of some of the location-wise soil variables that were tested in the modeling process. 


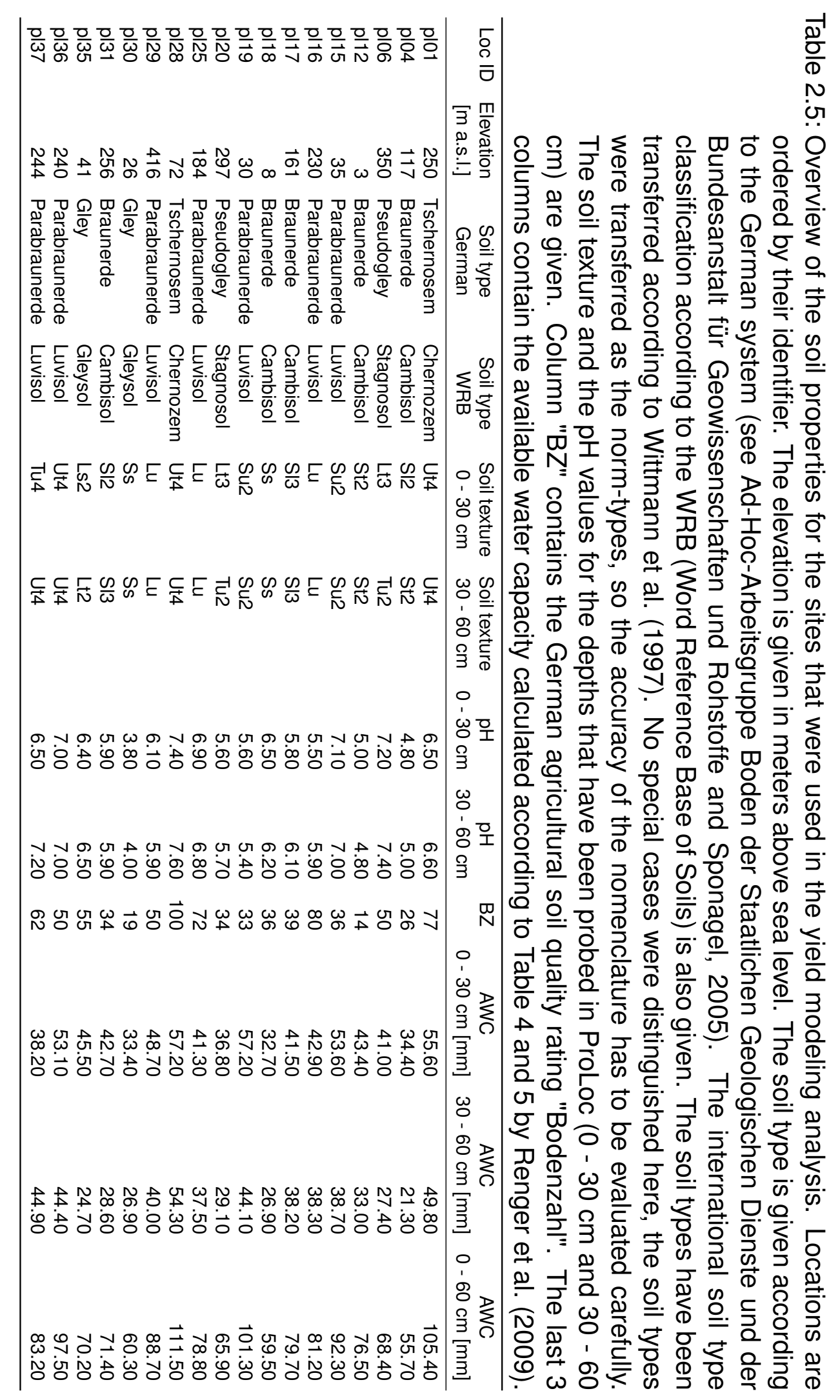




\section{Climatic data}

Climatic data was received from the German National Meteorological Service (Deutscher Wetterdienst, short: DWD). Daily sum of precipitation and mean temperature values were available from the DWD's own weather and climate stations. Due to the distances between these stations and the trial sites an interpolation of the daily climate data using the method by Schulla (2017) was performed. The interpolated daily data was aggregated for the analysis on different temporal scales ranging from a monthly basis to vegetation period, annual and rotation wise aggregation. The vegetation period (VP) for German forestry is defined as the time from May to September (Arbeitskreis Standortskartierung in der Arbeitsgemeinschaft Forsteinrichtung, 2016). The different monthly aggregations that were tested were gathered from prior publications by Ali (2009), Amthauer Gallardo (2014) and Hammes (1983). The last publication is based on physiological research on water use efficiency. The different aggregations were from April to July, May to June, May to July, June to July, July to August and July to September. During the modeling process it turned out that the monthly aggregations May to June for precipitation and June to July for temperature produced the best results in predicting growth. An overview on the correlation of these variables with the responses of the models that were parameterized is given in Section 2.2.1.

As described by Amthauer Gallardo (2014) in 2008 the trees on 7 trial sites were irrigated due to dry conditions after planting. Table 2.6 contains the mean sum of precipitation and mean temperature for the time periods May to June and June to July respectively. When sites were irrigated, the amount of water that was deployed is also given. It was incorporated into calculating the mean sum of precipitation. Furthermore, as an orientation, the climate summary data for the vegetation period is given. 
Table 2.6: Overview of the climate variables for the sites that were used in the yield modeling analysis. Locations are ordered by their identifier. The second column contains the mean annual sum of precipitation in May and June (Prec. 5-6) for the trial duration. The third column contains the amount of irrigation (Irrig. 5-6) that was applied to the respective sites within the first year during May and June (see Amthauer Gallardo, 2014). The fourth column contains the average of the mean monthly temperature in June and July for the whole trial duration (Temp. 56). The last two columns contain the mean sum of precipitation and mean monthly temperature for the vegetation period ranging from May to September.

\begin{tabular}{lrrrrr}
\hline Loc ID & Prec. 5-6 [mm] & Irrig. 5-6 [mm] & Temp. 6-7 $\left[{ }^{\circ} \mathrm{C}\right]$ & Prec. 5-9 [mm] & Temp. 5-9 $\left[{ }^{\circ} \mathrm{C}\right]$ \\
\hline pl01 & 182.80 & & 18.50 & 459.19 & 17.08 \\
pl04 & 156.80 & 19.00 & 369.49 & 17.55 \\
pl06 & 146.00 & 17.60 & 375.21 & 16.24 \\
pl12 & 114.40 & & 17.70 & 320.90 & 16.24 \\
pl15 & 117.70 & & 18.30 & 325.38 & 16.75 \\
pl16 & 142.20 & & 16.80 & 333.16 & 15.48 \\
pl17 & 136.80 & & 16.70 & 333.61 & 15.40 \\
pl18 & 116.10 & 11.00 & 17.00 & 313.65 & 15.69 \\
pl19 & 127.90 & 30.00 & 16.90 & 351.01 & 15.68 \\
pl20 & 130.30 & & 16.60 & 340.05 & 15.38 \\
pl25 & 134.00 & & 17.20 & 327.24 & 15.96 \\
pl28 & 125.20 & 0.90 & 18.00 & 329.36 & 16.63 \\
pl29 & 143.80 & & 16.30 & 349.21 & 14.93 \\
pl30 & 110.60 & 2.20 & 17.70 & 303.60 & 16.24 \\
pl31 & 164.70 & 5.00 & 17.70 & 441.59 & 16.36 \\
pl35 & 121.00 & & 16.80 & 342.71 & 15.50 \\
pl36 & 133.10 & & 16.90 & 332.39 & 15.53 \\
pl37 & 145.70 & & 17.40 & 356.85 & 16.04 \\
\hline
\end{tabular}




\subsubsection{Single Tree Based Forest Growth Modeling}

For implementing the growth simulator the idea was to build upon an existing implementation to minimize the effort in programming. At the department of Forest Growth at the NW-FVA a position independent growth model was parameterized and implemented within the software BWIN for a user-friendly access to the model (Hansen and Nagel, 2014). The model parameterization was based on data from forestry trial sites within the area of responsibility of the NW-FVA. After separating components of the software into own standalone programs the simulator for forest growth was renamed to BWINPro. The model was reimplemented as the Open Source project TreeGrOSS (Tree Growth Open Source Software) and re-written in the programming language Java (Hansen and Nagel, 2014). Several other applications use the functions within TreeGrOSS, the main focus here remains on the ForestSimulator. Within BWINPro tree growth refers to height and diameter increment (Hansen and Nagel, 2014). Species specific growth functions were parameterized that rely on independent variables like age, parameters relating to crown and its size and its change due to external factors like thinning or competition (Hansen and Nagel, 2014). This approach has advantages over classic yield tables especially in mixed stands (Pretzsch, 2010; Hansen and Nagel, 2014). For short rotation coppice some of the properties and the management regime of forest stands do not apply (at least not under conditions found in German forestry). SRC plantations are usually single species and even, which was also the case in ProLoc on the plot level, mono-clonal stands. Thinning methods, their impact on growth or aspects like future crop trees do not have to be considered because coppicing is practiced as clear-cut harvesting. Modeling growth has so far been mainly concerned with the stand level.

These differences already point towards the fact that some of the functionalities integrated into BWINPro and TreeGrOSS are not needed for implementing an SRC growth simulator. The main change or addition that has to be made though is the integration of resprouting meaning that a tree loses its above-ground biomass but, while still being alive, will start growing again after harvest. 
After reviewing the data from the ProLoc project and concluding on the underlying data generating processes the following modeling procedure was deemed feasible:

- Estimate the survival probability of single trees after planting

- Generate tree heights after planting based on environmental variables

- Estimate the growth of single trees during the first rotation cycle

- Estimate the survival probability of single trees after coppicing

- Estimate the growth during the vegetation period directly following the coppicing

- Estimate the growth of single trees during the second rotation cycle

The parameter that was focused on here for growth was tree height which was interpreted as the length of the tallest stem per tree. This parameter was measured in both rotation cycles while RCD was only measured in the first rotation. Single stem DBH was also measured in both rotations but due to the fact that below $1.3 \mathrm{~m}$ no $\mathrm{DBH}$ can be measured, problems exist by simulating growth in the examined young stands. Estimating single stem growth, especially after coppicing, was soon dropped from the modeling process because the stem data could not be matched between years since the methodology did not stipulate this from the beginning. Therefore single parameters per tree were utilized for growth. On these grounds the height increment as the length increment of the tallest stem was determined as the central variable. The scale of the environmental variables is the stand level. For the competition within stands however, an additional index had to be found. Due to the measurement grid in ProLoc the trees with available data were not directly neighboring. Therefore a distance-independent competition index had to be chosen. The basal area of the larger trees (short: BAL) was found to be a good index of the relative dominance per tree which also incorporates stand density (Gadow, 2003). For calculating this index, the basal area per tree needs to be determined first. This was realized by calculating the basal area of each stem per tree. In the first rotation the RCD was utilized as the required 
diameter, in the second rotation this was switched to the DBH due to the available data. The BAL is then calculated, according to Gadow (2003), based on the cumulative frequency of basal area.

This necessitates calculating the basal area of the stand $G$ in square meter per hectare as

$$
G=\sum_{i=1}^{n} g_{i}\left[m^{2} h a^{-1}\right]
$$

where $g_{i}$ is the tree-wise basal area (sum of basal area of single stems) of tree $i$. The cumulative basal area frequency is then

$$
p_{j}=1-\frac{G G_{i j}}{G_{i}}
$$

where $G_{i}$ is the total basal area of the stand $i$ in $m^{2} h a^{-1} . G G_{i j}$ is the sum of the basal areas of all trees with diameters larger than the reference tree $j$ which is calculated as

$$
G G_{i j}=G_{i}\left(1-p_{j}\right)
$$

(All last three formulas taken from Gadow (2003, p. 98)). An increasing value for the BAL indicates a decreasing relative dominance of the tree since there are increasingly more trees with a larger basal area. Using other competition indices was either not feasible or did not yield satisfactory results, as it was the case for relative height in relation to mean stand height or relative height rank within a stand. Since growth was mainly focused on height increment, basal area per tree had to be estimated by height which will be described as a part of the simulator inherent data completion routines (see Sections 2.2.3 and 2.2.4).

Apart from the integrated data completion, some of the datasets within the increment data had to be completed before modeling. This relates mainly to the higher number of diameter measurements in relation to height measurements because of the measuring grid. The methodology of how this was conducted is described in the next section. 


\section{Stand Height Curves}

To complete the missing height observations height-diameter equations (also called height curves) were parameterized to predict height based on RCD or DBH. For TreeGROSS Hansen and Nagel (2014) use a nonlinear approach applying five functions listed by Schmidt (1967). The functions and properties in TreeGrOSS have not been parameterized for poplar and willow, instead the parameterization for beech and oak with the Petterson function is used. TreeGrOSS requires a minimum of five complete height-diameter value pairs for the parameterization of the Petterson function per imported stand object. If the required number of observations is not met, a uniform height curve is used instead. Hartmann (2010) compared the goodness of fit for seven different equations for estimating height from diameter for poplar in SRC plantations. These equations include the 5 functions used by Hartmann (2010); Hansen and Nagel (2014) identifies the Petterson function as the most suitable equation in terms of goodness of fit assessed by the coefficient of determination $R^{2}$. 25 pairs of values are stated as the required $n$ for the plantation wise parameterization of the height curve. Skibbe (2016) assessed the Prodan equation as the model with the best fit for willow. The required sample size was proposed as 15 pairs of values.

Both function types Petterson and Prodan have the advantage of possible linearization allowing for the application of linear model and linear mixed-effects model theory. Schmidt et al. (2011) have demonstrated the advantages of this procedure and implemented a height-diameter model for Pinus sylvestris L. in Estonia. Equation 2.5 contains the nonlinear formulation of the Petterson function (also known as Näslund function (Schmidt et al., 2011)):

$$
h_{i j}=\left(\frac{d_{i j}}{\alpha+\beta d_{i j}}\right)^{\gamma}+1.3
$$

with $h_{i j}$ as the single tree height of the $i$ th tree within the $j$ th plot. $\alpha, \beta$ and $\gamma$ are parameters whose values determine the curve progression and are optimized during nonlinear least squares fitting. The equation here is adapted from Schmidt et al. (2011) who have also included the temporal scale into the hierarchy (additionally to plot and single tree). For the linearization Schmidt et al. (2011)) fixed the exponent $\gamma$ to 3 in accordance to Kramer and Akça (2008). This yields the Equation 2.6 


$$
y_{i j}=\frac{d_{i j}}{\left(h_{i j}-1.3\right)^{(1 / 3)}}=\alpha+\beta d_{i j}
$$

Based on the single tree height $h$, the diameter $d$ and $\gamma=3$, a new response $y$ can be calculated and then used in linear modeling. The nonlinear version of the Prodan function is given in Equation 2.7:

$$
h_{i j}=\frac{d_{i j}^{2}}{\alpha+\beta d_{i j}+\gamma d_{i j}^{2}}+1.3
$$

The definition for the indices $i$ and $j$ and the coefficients $\alpha, \beta$ and $\gamma$ is the same as for the Näslund function above. The Equation is adapted from Schmidt (1967) who also establish the linearized version as adapted here in Equation 2.8.

$$
y_{i j}=\frac{d_{i j}^{2}}{h_{i j}-1.3}=\alpha+\beta d_{i j}+\gamma d_{i j}^{2}
$$

For the ProLoc data the two described linearized functions were tested additionally to using a simple second-degree polynomial for diameter with an untransformed response, transforming just the response with the Box-Cox procedure or using a double log transformed function (log transform for response and independent variable diameter). Here the linearized Näslund function produced the best results in comparison. The comparison of the different approaches will not be further discussed here. The Petterson/Näslund function was modified for the root collar diameter since it is measured at $0.1 \mathrm{~m}$ and not $1.3 \mathrm{~m}$ as the diameter at breast height. The coefficient $\gamma$ was set to 2.5 here which yielded better results than 2. A power of 3 did not improve the overall fitting procedure. Within the first rotation height as the maximum stem length was estimated by maximum root collar diameter per tree.

For all years, sites and clone combinations, mixed-effects models were fitted with the response from the linearization, the tree-wise maximum RCD or maximum DBH as the independent variable and a random effect for plots within the completely randomized design. At first, the performance of a single model that encompasses all described hierarchies and incorporates a correlation structure between years was tested. The results were not satisfying, so the approach with separate height curve models was chosen. For site pl18 Guelzow no functions needed to be parameterized because both variables had always been measured. 
Table 2.7 gives an overview on the quantity of completed datasets after the described completion.

Table 2.7: Overview of the number of diameter and height value pairs after the data completion. The differing numbers between stands are due to lower survival rates and the fact that not all clones were included in all locations. Increasing numbers between years are based on the fact that in the first rotation dead trees were switched with neighboring alive trees. In the sixth year on some trials full plots were measured for testing different MAl estimation methodologies which also implies an increase in datasets.

\begin{tabular}{lrrrrrr}
\hline Loc ID & Year 1 & Year 2 & Year 3 & Year 4 & Year 5 & Year 6 \\
\hline pl01 & 476 & 486 & 484 & 304 & & 269 \\
pl04 & 467 & 479 & 474 & 270 & 262 & 259 \\
pl06 & 261 & 287 & 291 & 282 & 191 & 191 \\
pl12 & 488 & 484 & 477 & 448 & 296 & 316 \\
pl15 & 456 & 897 & 481 & & 288 & 288 \\
pl16 & 382 & 370 & 370 & & 240 & 240 \\
pl17 & 480 & 480 & 480 & 482 & 320 & 478 \\
pl18 & 160 & 161 & 160 & & 284 & 272 \\
pl19 & 374 & 373 & 371 & 223 & 215 & 214 \\
pl20 & & 471 & 475 & 420 & 275 & 272 \\
pl25 & 481 & 481 & 478 & & 303 & 310 \\
pl28 & 472 & 472 & 472 & 435 & 294 & 299 \\
pl29 & 477 & 477 & 465 & 433 & 308 & 308 \\
pl30 & 469 & 464 & 464 & 227 & 193 & 201 \\
pl31 & 483 & 479 & 486 & 452 & 301 & 300 \\
pl35 & 523 & 463 & 470 & 464 & 270 & 267 \\
pl36 & 382 & 382 & 382 & 372 & 253 & 253 \\
pl37 & 384 & 384 & 384 & 289 & 256 & 245 \\
\hline
\end{tabular}

Empty cells indicate no measurements which occurred, except for stand pl20 in the first year, mostly for the fourth year and because of uncertainties regarding the continuation of the project. For growth modeling the datasets were checked for plausibility. Especially for height increment some entries were dropped due to implausibility which were most likely due to measurement error or misalignment of trees between years (data entry errors). 


\section{Weibull Distribution}

For generating tree heights after planting within the simulation process a function was needed that approximates the distribution of height values in the original data. The Weibull distribution is commonly utilized as a frequency distribution in forest growth modelling (Nagel and Biging, 1995; Gadow, 2003; Fabrika and Pretzsch, 2013). Fabrika and Pretzsch (2013) indicate its adaptability and convenient interpretation as the reason for its popularity, which is backed by the integration into 3 forest growth simulators, including BWINPro (Hansen and Nagel, 2014). The parameterization for the latter is described by Nagel and Biging (1995). A 2 parameter form of the Weibull function was used, fixing the location parameter to $\alpha=0$. Furthermore the function was truncated to incorporate a threshold value of $T=7 \mathrm{~cm}$ for the $\mathrm{DBH}$, omitting smaller trees. Following Nagel and Biging (1995) the 2 parameter distribution function is defined as

$$
g(x)=\frac{c}{b}\left(\frac{x}{b}\right)^{c-1} \exp \frac{T^{c}-x^{c}}{b^{c}}
$$

with $g(x)$ as the absolute frequency of trees with variable $x(x \geq 0), b$ as the scale parameter and $c$ as the shape parameter (Formula see Nagel and Biging, 1995, p. 185). The two parameters for shape and scale were estimated based on stand level data collected in survey plots in northwest Germany. The database for this analysis consisted of an overall number of 2242 measurements in 450 plots including 6 tree species. Regression analysis indicated a strong linear relationship between the scale parameter $b$ and mean DBH resulting in a coefficient of determination $R^{2}>0.95$ for all tree species. For estimating the shape parameter $c$ the mean diameter of the 100 thickest stem and the maximum diameter were used as predictors with $R^{2} \geq 0.6$. With the estimated shape and scale parameter as well as the inverse function of the cumulative frequency distributions random $\mathrm{DBH}$ values based on the parameterized Weibull distribution can be generated (Nagel and Biging, 1995). This is defined as

$$
D B H=b *\left[\left(\frac{T}{b}\right)^{c}-\log _{e}\left(1-F_{T}(x)\right)\right]^{\frac{1}{c}}
$$

with $F_{T}(x)$ being the cumulative frequency diameter distribution (Formula see Nagel and Biging, 1995, p. 187). Generating a uniform random number between 
0 and 1 and applying it to this function produces a DBH value from the fitted distribution which is larger than the selected threshold. For generating the height data, the same methodology was used. Only the threshold value of $T$ was changed to $0.1 \mathrm{~m}$. For each trial plot with data from the first year the Weibull distribution was fitted to the height values. All fits were tested separately via the KolmogorovSmirnov test to determine if the distribution of sample heights differed significantly from the Weibull distribution. Shape and scale parameters were then extracted from the fits and used for further regression by matching it with the mean stand height for each plot.

\subsubsection{Software and Statistical Analysis}

\section{Statistical Methodology}

Assessing the experimental design in ProLoc a decision had to be made if the different spatial hierarchies should be incorporated into growth modeling as nested random effects in the framework of mixed-effects modeling. The different scales can be defined as location, plot within location and single tree within plot. These can also be viewed as strata following the terminology of Williams et al. (2002). Incorporating random effects can improve the model fitting due to a better approximation of the experimental design at hand and accounting for sources of variance. Based on these reasons mixed effects models were employed for the analysis by Janßen et al. (2017) with plot level data. It can be seen as problematic though to incorporate the location as a random effect and then use location-wise environmental variables as fixed effects. As a general consideration of including site as a random effect in multilocation trials, Piepho et al. (2003) elaborate on the issue of treating the location factor as fixed or random. In agricultural trials the sampling of trial locations is often not random in a strict sense because location selection relies on available fields close to existing research facilities which were often placed themselves to represent certain environmental conditions (Piepho et al., 2003). Piepho et al. (2003) recommend to treat location not as random but as fixed in these cases. It was decided to follow this recommendation in the modeling process here because the allocation of factor location can not be seen as random in ProLoc. The trial sites and supervising cooperation partners were 
rather chosen for a wide range of environmental conditions. In contrast, the factor plot within site can be seen as random due to the randomized assignment of clones to the different plots. The only instance were this was taken into consideration was, as already described, for the parameterization of the linearized stand height curves.

The significance level for all tests was fixed to a value of $\alpha=0.05$. The $p$ values in the model summaries are rounded to 3 digits and are printed as $<0.001$ below 0.001 .

For modeling any relationship between a dependent and one or multiple independent variables it was first evaluated if the methodology of simple linear regression could be applied. The emphasis was put on the latter due to its well established theoretical foundations and application (Faraway, 2015). With fitting a linear model the assumptions for the application of these were checked first. This includes that the errors $\epsilon$ have mean 0 , that the error variance $\sigma^{2}$ is constant (homoscedasticity) and that the errors are uncorrelated (Fahrmeir et al., 2013). These assumptions were checked with regression plots or violations could be eliminated on basis of the data at hand. The latter refers for example to the height increment modeling in the first and second rotation where it's obvious that due to repeated measurements on the same trees some correlation will trivially be present. If, however, all assumptions were met, and a linear model could be parameterized the summary of this model will be given in the following Sections in a uniform way. Along with the estimated standard error of the residuals $\hat{\sigma}$ the results of a comparison of the fitted with a null model will be given for assessing the goodness of fit. The null model is either a model with fitted intercept (mean model) or no intercept implying that the intercept/mean is 0 . The differentiation between these two cases depends on whether the linear model was fit with an intercept or not. In both cases the residual sum of squares and the degrees of freedom of the null $\left(R S S_{\text {Null }}, d f_{\text {Null }}\right)$ and the fitted model $\left(R S S_{\text {Model }}, d f_{\text {Model }}\right)$ are given. These can then be used to calculate the $F$-statistic for comparing it with the $F$ distribution. The null hypothesis of this test is $H_{0}: \beta_{1}=\ldots \beta_{p-1}=0$ where $\beta_{i}$ are the regression parameters and $p-1$ is the number of parameters (Faraway, 2015, p. 35). The $p$ value of this test is also given in the model summary for convenience. For assessing the goodness of fit, the $R_{a d j}^{2}$ is presented if the model was fitted with an intercept, while, without an intercept, based on the 
recommendation by Faraway (2015), the squared correlation of estimated and observed values $\operatorname{cor}^{2}(\hat{y}, y)$ is given. Following the general model summary, for each single covariate of the parameterized linear model the coefficient (Est.) is given together with its standard error se. Each coefficient was tested via $t$-test for the null hypothesis $H_{0}: \beta_{i}=0$ (Faraway, 2015, p. 37). The $p$ values are also reported for these tests.

It was generally refrained from using nonlinear approaches and rather tried to transform the response or add polynomial terms on the side of independent variables if a relationship was obviously nonlinear and therefore the assumptions for a linear model were not fulfilled. For identifying the optimal transformation of the response the Box-Cox procedure was used (see Venables and Ripley, 2010; Faraway, 2015). In many cases this could solve the problems of nonlinearity and/or heteroscedasticity. Fischer (2016) has also proven the advantages of using the Box-Cox procedure in forest growth modeling. If the response of a model was transformed, it is stated in the caption of the model diagnostics table. The shown diagnostics are based on the model with the transformed response and are given in its scale. In simulating growth, the estimated values were then backtransformed. No correction for transformation bias was applied here.

In some cases the transformation procedure did not yield satisfying results in eliminating heteroscedasticity or, as already stated, there were problems due to serial autocorrelation based on repeated measurements. In these cases a linear model using generalized least squares (short: GLS) was fitted. For some theoretical background on these the reader is referred to Pinheiro and Bates (2000). The variance power function with parameter $\delta$ can account for increasing or decreasing variance with increasing estimated values. If a linear model with GLS using the variance power function was fitted, $\hat{\delta}$ is printed in the general model diagnostics tables. For serial autocorrelation an autoregressive correlation structure of order 1 was used. The correlation parameter $\Phi$ equals 0 by default and the estimated parameter value $\hat{\Phi}$ ranges between -1 and 1 (Pinheiro and Bates, 2000). $\hat{\Phi}$ is also given in the model summary diagnostics of linear models using GLS. As an omnibus test for goodness of fit the fitted model is compared via likelihood ratio test to a null model. For each model the log-likelihood $l l$ is reported along with its degrees of freedom referring to the number of parameters in the model here (Pinheiro and Bates, 2000). Additionally the test statistic of the likelihood ratio test 
$(L R T)$ is presented in the model diagnostics with a corresponding $p$ value. The linear models using GLS were by default fitted using the restricted maximum likelihood method. For the likelihood ratio test, the fitted model had to be refitted (see Pinheiro and Bates, 2000) using the maximum likelihood fit and the null model was also fitted in this way. Again, for further theoretical background the reader is referred to Pinheiro and Bates (2000). The goodness of fit was assessed by calculating the squared correlation of fitted and observed values $\operatorname{cor}^{2}(\hat{y}, y)$. The diagnostics for the single covariates and their coefficients are the same as for the linear model.

For modeling binary coded variables and count data generalized linear models (short: GLM) were employed. For example, regarding the survival rate a tree being alive is seen as a success (coded as 1 ) and a tree being dead is seen as a failure (coded as 0 ). GLM were preferred to the otherwise common approach of transforming the survival rates with the arcsine square root transformation (see Thomas, 2006). Warton and Hui (2011) elaborate on the reasons for preferring the GLM approach to transforming survival rates. For binary coded and binomially distributed response the binomial model family was used, for count data the poisson model family was employed. Inherent to the GLM implementation in $\mathrm{R}$ is that the response is estimated through link functions. The response $\eta$ for the binomial model with the logit link function is given as follows (adapted from Faraway, 2016, p. 28):

$$
\eta_{i}=\log \left(\frac{p_{i}}{1-p_{i}}\right)
$$

where $\eta$ is the response of the link function that is being estimated by the GLM and $p$ is the probability of a success. Based on the response the probability can be calculated as $p_{i}=\frac{e^{\eta_{i}}}{1+e^{\eta_{i}}}$ (adapted from Faraway, 2016, p. logit).

For the poisson model family the log link function was used which simply results in (adapted from Faraway, 2016, p. 86):

$$
\eta_{i}=\log \left(\mu_{i}\right)
$$

Trivially, the mean count response $\mu_{i}$ is back-transformed as $\mu_{i}=e^{\eta_{i}}$. The coefficients that are given in the GLM model summaries have to be interpreted regarding the response of the respective link function. If allowing for more vari- 
ance in the response than expected was necessary, quasi-likelihood estimators were used in the modeling process. For a theoretical background see Faraway (2016). The model diagnostics for GLM contain the model family and the link function that was used. If the quasi-likelihood procedures were used, the estimated dispersion parameter $\Phi$ is also given. Comparably to the linear models the goodness of fit was examined by comparing the fitted model to a null model. For both models the respective deviance $D$ and the degrees of freedom $d f$ are given. Based on this, the models were compared by a $\chi^{2}$-test for standard GLM or an $F$ test for GLM accounting for overdispersion. As a measure to compare goodness of fit between generalized linear models the amount of deviance left unexplained by the model in regard to the null model's deviance, expressed as $D^{2}$, was used. The $D^{2}$ statistic can have values between 0 and 1 and is interpreted like the more commonly known $R^{2}$ for linear models. For the GLM that were parameterized the $\mathrm{R}$ implementation of $D^{2}$ by Barbosa et al. (2016) was applied following the definitions of Guisan and Zimmermann (2000). This includes the following formula (Guisan and Zimmermann, 2000, p. 166-167, formula 1-2):

$$
\begin{gathered}
D^{2}=\frac{\text { Null deviance }- \text { Residual deviance }}{\text { Null deviance }} \\
D_{a d j}^{2}=1-\frac{n-1}{n-p} *\left(1-D^{2}\right)
\end{gathered}
$$

Comparable to the $R_{a d j}^{2}$ the latter statistic $D_{a d j}^{2}$ takes the number of observations and predictors into consideration. Faraway (2016) suggests using the $R^{2}$ method by Nagelkerke (1991) instead of this method, though the overall reliability of these goodness of fit statistics for GLM is criticized. The given $D_{a d j}^{2}$-values should be interpreted carefully and are given rather for orientation.

In additon to the model summaries, the model functions are also given for a better overview when at least 3 independent variables (excluding polynomial terms) are present in the parameterized model.

When parameterizing any of these kind of models a procedure has to be employed to identify and select the variables that have a significant influence on the response. These are often non-trivial decisions which can be ambiguous (Faraway, 2015). Special attention was given towards the fact that correlation does not always imply causation. To aid the decision process a stepwise vari- 
able selection with backward elimination and comparing models by the Bayes information criterion BIC (see Faraway, 2015; Venables and Ripley, 2010) was chosen. Based on evaluating the biological, data generating processes and the available data the independent variables were chosen for each model. If reasonable, their interactions were also added to the model. This was only realized for two-way interactions for easier interpretability. The significant influence of single covariates was also checked with the tests described above and analysis of variance procedures that were applied to the model. For the latter the type III sums of squares were used because in most cases, and especially for the factor clone, the data was unbalanced. This methodology is seen as more reliable in unbalanced datasets (Fox and Weisberg, 2019). With these tests and procedures a suitable set of predictors was identified. The coefficients were then checked for plausibility. In some cases variables were dropped from the selection procedure if the produced results were implausible. If predictors had a significant but practically marginal influence, they were dropped from the model if the predictive power did not suffer from the omission. The motivation behind this was identifying the best fitting and, in terms of number of independent variables, parsimonious model.

The former statements relate to the inferential statistics. The descriptive statistics in the following sections should be self explanatory. Only a remark towards the abbreviations used in boxplots should be made. Above each category within the boxplot a short numerical summary is given. This includes the number of observations $n$, the arithmetic mean $M$ and the standard deviation $s$.

\section{Utilized Software}

In the following paragraph the utilized software for statistical analysis and simulator implementation is listed. The versions of the software and packages included here and in the bibliography are the latest releases that were used. Of course, during the course of analyzing the data for this thesis versions may have changed due to updates but the presented results were obtained using the versions given here or in the bibliography. The statistical analysis was carried out using the programming language $R$ ( $R$ Core Team, 2019, version 3.6.0) within the development environment RStudio (RStudio Team, 2016, version 1.1.463). For linear models and generalized linear models the standard $R$ functions $1 \mathrm{~m}$ respectively glm from 
the integrated stats package were used. For the GLS estimation (program function gls), the variance power function (varPower) and the correlation structure (corAR1) the nlme package was used (Pinheiro et al., 2019, version 3.1-139). As already indicated, for the calculation of the explained Deviance $D$ the implementation of the modEvA package was used (Barbosa et al., 2016, version 1.3.2). The routine for the Box-Cox procedure and for the stepwise variable selection was inferred from the MASS package (Venables and Ripley, 2010, version 7.3-51.4). The type III sum of squares analysis of variance was provided through the car package (Fox and Weisberg, 2019, version 3.0-2).

Here it should be noted that the factor clone, being the only categorical factor variable in the modeling process, was usually entered into the model using R's default treatment contrast with the function contr.treatment. This means that the clone 'Max 1' is at the base level and the coefficients for the other factor levels (being the other clones) can be interpreted as the differences towards 'Max 1'. This might be counterintuitive at first but in comparison to the other contrast options the default method is easier to interpret and the method of coding a factor plays no role for the analysis in most cases (Fox and Weisberg, 2019). Only for the type III sum of squares analysis of variance the Helmert contrasts through the function contr. helmert were used because they are more appropriate in this context (see Fox and Weisberg, 2019).

For general data manipulation the different packages subsumed under the tidyverse package (Wickham, 2017, version 1.2.1) were used. Visualizations were created using the ggplot2 package (Wickham, 2016, version 3.1.1). Tables were exported from $\mathrm{R}$ to Latex via the xtable package (Dahl et al., 2019, version 1.8-4).

For implementing the growth simulator the integrated development environment IntelliJ IDEA was used (IntelliJ Developer Team, 2019, version 2019.1.3). 


\subsection{Results}

\subsubsection{Correlation of Environmental Data and Growth}

Before describing the fitted models for the single tree based growth simulator, a short description of the correlation of environmental variables with growth parameters can be helpful. In the following section the correlation that is being referred to stands for the Spearman correlation coefficient. The latter was chosen here because the relationship could not always be assumed as linear. The responses were chosen here corresponding to the modeling procedure laid out in Section 2.1.5. For survival models the survival percentage was arcsine square root transformed (see Thomas, 2006) for simplicity. Figure 2.4 contains a display of all Spearman correlations between the responses of the growth models and selected soil parameters. Only indicators of general soil quality like the German agricultural soil quality rating (Bodenzahl: BZ) and soil water storage indicators in form of the available water capacity, calculated according to different methodologies, are presented here. Regarding the latter, the KA5 standard (Ad-Hoc-Arbeitsgruppe Boden der Staatlichen Geologischen Dienste und der Bundesanstalt für Geowissenschaften und Rohstoffe and Sponagel, 2005) relating to the soil depth 0 to $60 \mathrm{~cm}$ and the changes suggested by Renger et al. (2009) relating to the same depth and additionally relating to the effective rooting depth were used.

The overall impression is that the correlation coefficients are not too high here. The highest values were reached for available water capacity and mean stand height after the first year $l$ Mean $Y 1$ with 0.54 . It should be minded though that the other responses incorporate a higher number of causes for variance because the single tree level is considered here. Considering this framework, the soil quality rating $B Z$ shows comparably high correlation with most responses except survival after resprout surv $Y$ 4. The available water capacity variables all show a higher correlation in the first rotation for estimating the mean stand height after the first rotation. The available water capacity calculated according to Renger et al. (2009) and relating to the soil depth of 0 to $60 \mathrm{~cm}$ has the highest correlation with growth responses in the first rotation. All 3 available water capacity variables have in common though that their correlation coefficients are lower with growth responses in the second rotation (incr $H Y 4$ and incr $H R 2$ ). 


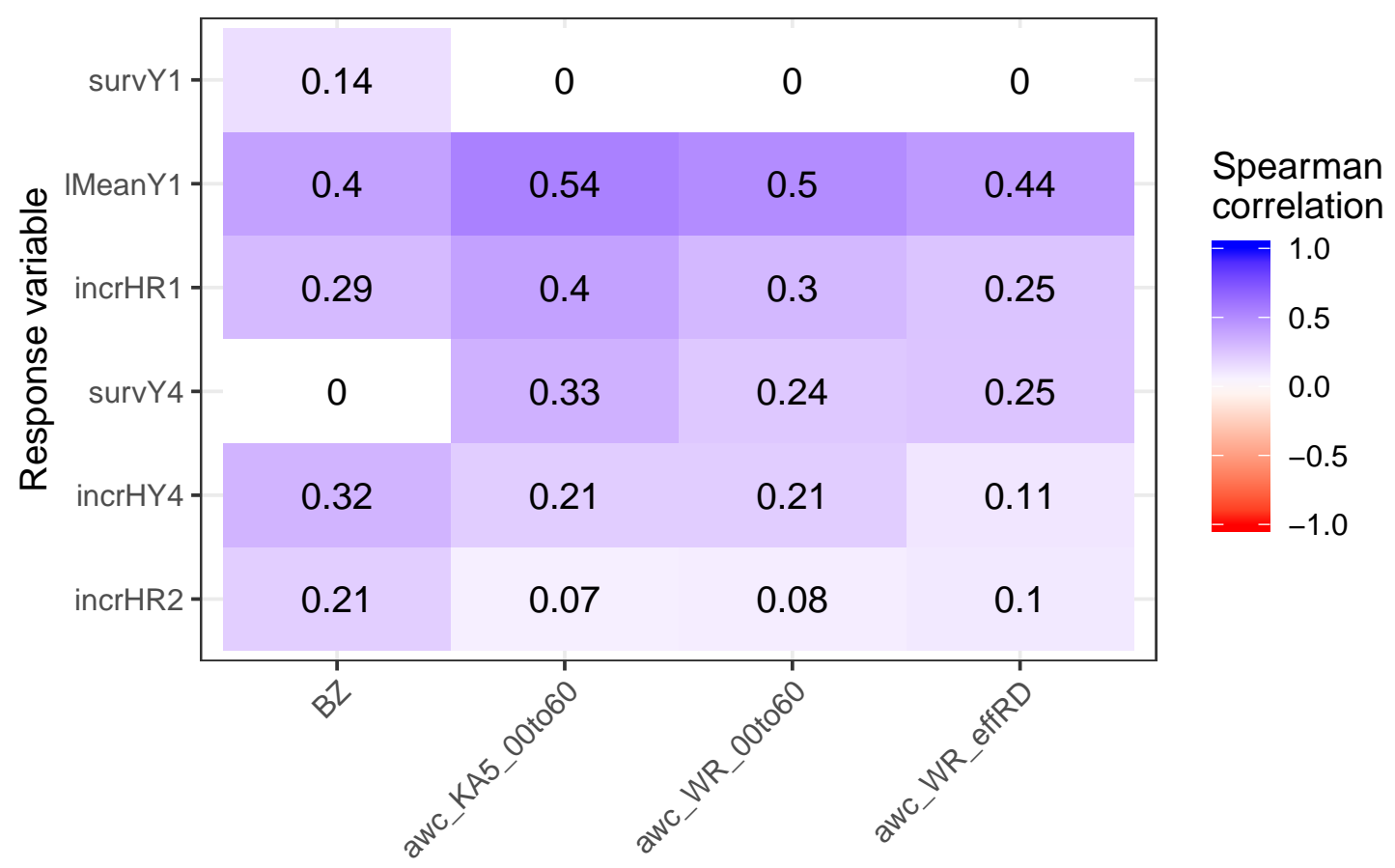

Soil variable

Figure 2.4: Spearman correlation between the responses of all 6 growth models and 4 soil variables. The magnitude of the correlation is indicated by a color gradient. If the $p$-value of the correlation test indicated that the correlation coefficient did not significantly differ from 0 , the value was set to 0 for the graph. The responses are survival at the end of the first year after planting (surv $Y 1$ ), mean stand height at the end of the first year after planting (lMeanY 1$)$, height increment within year 2 and 3 during the first rotation (incr $H R 1$ ), survival after coppicing at the end of the fourth year ( $\operatorname{surv} Y 4$ ), the height growth after coppicing at the end of the fourth year (incr HY 4) and height increment within year 5 and 6 during the second rotation (incr $H R 2$ ). The soil variables are the Bodenzahl $(B Z)$, the available water capacity in soil depth 0 to $60 \mathrm{~cm}$ (awc_KA5_00to60) calculated according to Ad-Hoc-Arbeitsgruppe Boden der Staatlichen Geologischen Dienste und der Bundesanstalt für Geowissenschaften und Rohstoffe and Sponagel (2005), the available water capacity in soil depth 0 to $60 \mathrm{~cm}$ (awc_WR_00to60) calculated according to Renger et al. (2009) and the available water capacity in the effective rooting depth $\left(a w c \_W R \_\right.$ef $\left.f R D\right)$ calculated according to Renger et al. (2009). 
In Figure 2.5 the focus is shifted from soil to climate by displaying the Spearman correlation between the responses of the same growth and survival models and climate variables aggregated by different time spans. The monthly aggregations that are used here were gathered from publications that established their influence on growth. While different aggregations were chosen for temperature and precipitation by Amthauer Gallardo (2014) and Ali (2009), here all aggregations were tested for both precipitation and temperature. The former in the form of mean annual sum of precipitation and the latter as mean of the annual mean temperature in the respective period.

Overall, the correlation coefficients values are on a comparable level as it was the case for the soil variables. The highest values were reached for mean stand height after the first year $l M e a n Y 1$ and mean sum of precipitation from April to July and May to June. The correlations between growth responses and precipitation are mainly positive and for temperature mainly negative which is expectable. Focusing on precipitation, the survival models surv $Y 1$ and surv $Y 4$ seem to be less closely correlated with the precipitation variables tested here. This is also the case for growth within the second rotation incr HR2. Comparing the different monthly aggregations, the time period May to June which was taken from Ali (2009) performs best. Within the temperature facet in Figure 2.5 it is of interest that some of the aggregations focusing on time spans early in the vegetation period have a positive correlation with mean stand height and height increment in the first rotation. The latter is less distinctive and might originate from the correlation of $l M e a n Y 1$ and incr $H R 1$ to some degree. The temperature in the same period has nevertheless an adversary effect on the survival. This was not further investigated as the full model parameterization and variable selection pointed towards the temperature in June and July as suited best for prediction.

Generally, it should be minded here that the description in this Section only gives a first impression on how single variables are correlated with each other while for the final growth models multiple covariates and their interaction were tested for their influence on the response which can produce differing results. These are described in the next Section. 


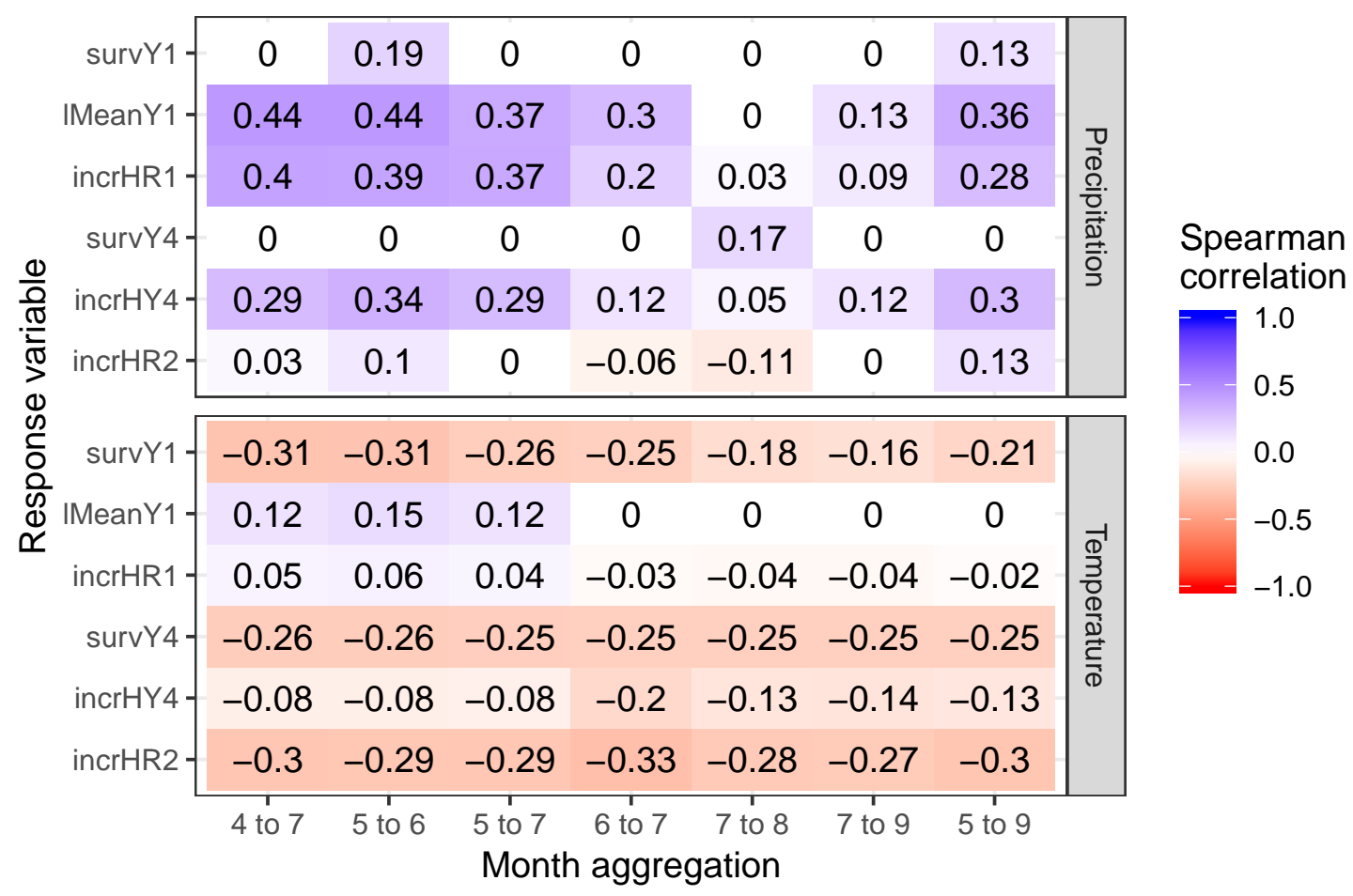

Figure 2.5: Spearman correlation between the responses of all 6 growth models and 7 different temporal aggregations for climate variables. The magnitude of the correlation is indicated by a color gradient. If the $p$-value of the correlation test indicated that the correlation coefficient did not significantly differ from 0 , the value was set to 0 for the graph. The responses are survival at the end of the first year after planting (survY1), mean stand height at the end of the first year after planting (IMeanY1), height increment within year 2 and 3 during the first rotation (incrHR1), survival after coppicing at the end of the fourth year (survY4), the height growth after coppicing at the end of the fourth year (incrHY4) and height increment within year 5 and 6 during the second rotation (incrHR2). The climate variables are aggregated for different months and time spans according to Amthauer Gallardo (2014), Ali (2009) and Hammes (1983). Additionally the time span of the forestry vegetation period from May to September according to Arbeitskreis Standortskartierung in der Arbeitsgemeinschaft Forsteinrichtung (2016) is given. Temperatures are given as mean values over the trial period, precipitation is given as the mean sum of precipitation, also over the trial period. 


\subsubsection{Data Completion with Stand Height Curves}

Before modeling the growth parameters for the single tree based growth model, the dataset had to be completed since not all increment variables were measured on all trees. The methodology as described in 2.1.5 was applied utilizing the linearized Näslund function for each combination of site, year and clone.

Figure 2.6 allows for a comparison of the observed and estimated height values within the first rotation gained with the described procedure.
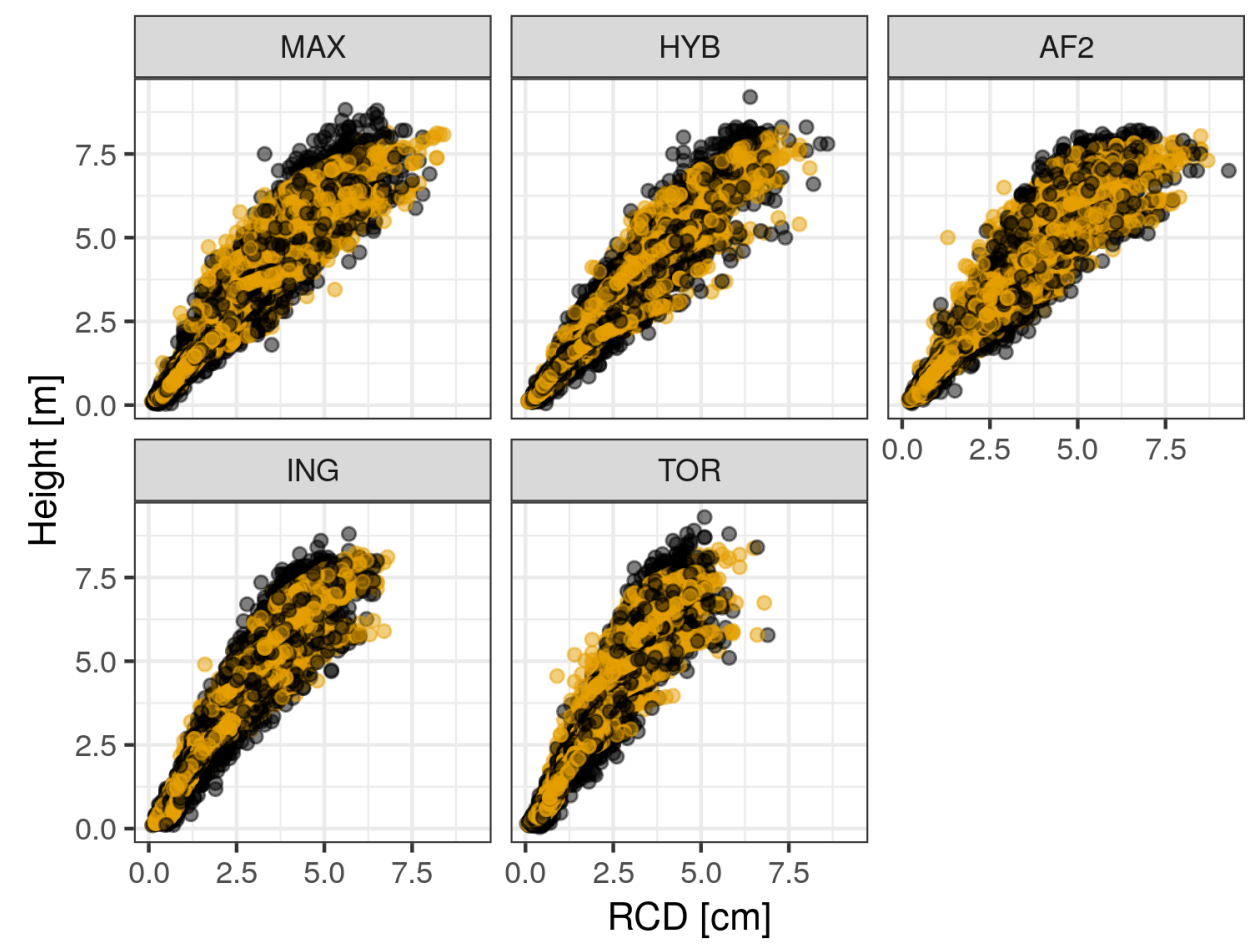

Source

- obs.

est.

Figure 2.6: Relationship of tree height as maximum stem length and maximum root collar diameter (RCD) during the first rotation separated by clone. Shown are the observed (obs., black points) and estimated height values (est., based on RCD, yellow points).

The overall goodness of fit, just by visual assessment, can be seen as good. The general impression is that the curves are relatively steep which is typical for younger stands. The poplar clones tend to have a similar, less steep curve progression in contrast to the steeper curve progression of the willow clones. The poplar clones reach higher RCD values than both willow clones while maximum height values are more similar. 
Table 2.8 gives an overview on the squared Pearson correlation between observed and estimated values for all 268 parameterized models within the first rotation. While there are few exceptions with a poorer fit the overall predictive power can be evaluated as very good.

Table 2.8: Summary of the distribution of squared Pearson correlation values $\operatorname{cor}(\hat{y}, y)^{2}$ between estimated $\hat{y}$ and observed $y$ values for height estimation by root collar diameter (RCD) and diameter at breast height $(\mathrm{DBH})$. Given is an extended five number summary with minimum/maximum value (Min, Max), the lower and upper quartile $\left(Q_{0.25}\right.$, $\left.Q_{0.75}\right)$, the median $(M d n)$ and the mean $(M)$. Values have been rounded to 2 digits.

\begin{tabular}{lccccccc}
\hline Independent variable & $n$ & Min & $Q_{0.25}$ & $M d n$ & $M$ & $Q_{0.75}$ & Max \\
\hline RCD & 268 & 0.02 & 0.91 & 0.96 & 0.90 & 0.98 & 1.00 \\
DBH & 272 & 0.09 & 0.93 & 0.97 & 0.92 & 0.98 & 1.00 \\
\hline
\end{tabular}

For assessing the predictive power of the approach to estimate the missing height values in the second rotation by diameter at breast height (DBH), the same summary is also given for DBH as the independent variable. The impression here is similarly good as for RCD with high correlation coefficients between observed and estimated values. The visual assessment can be repeated here in Figure 2.7. 


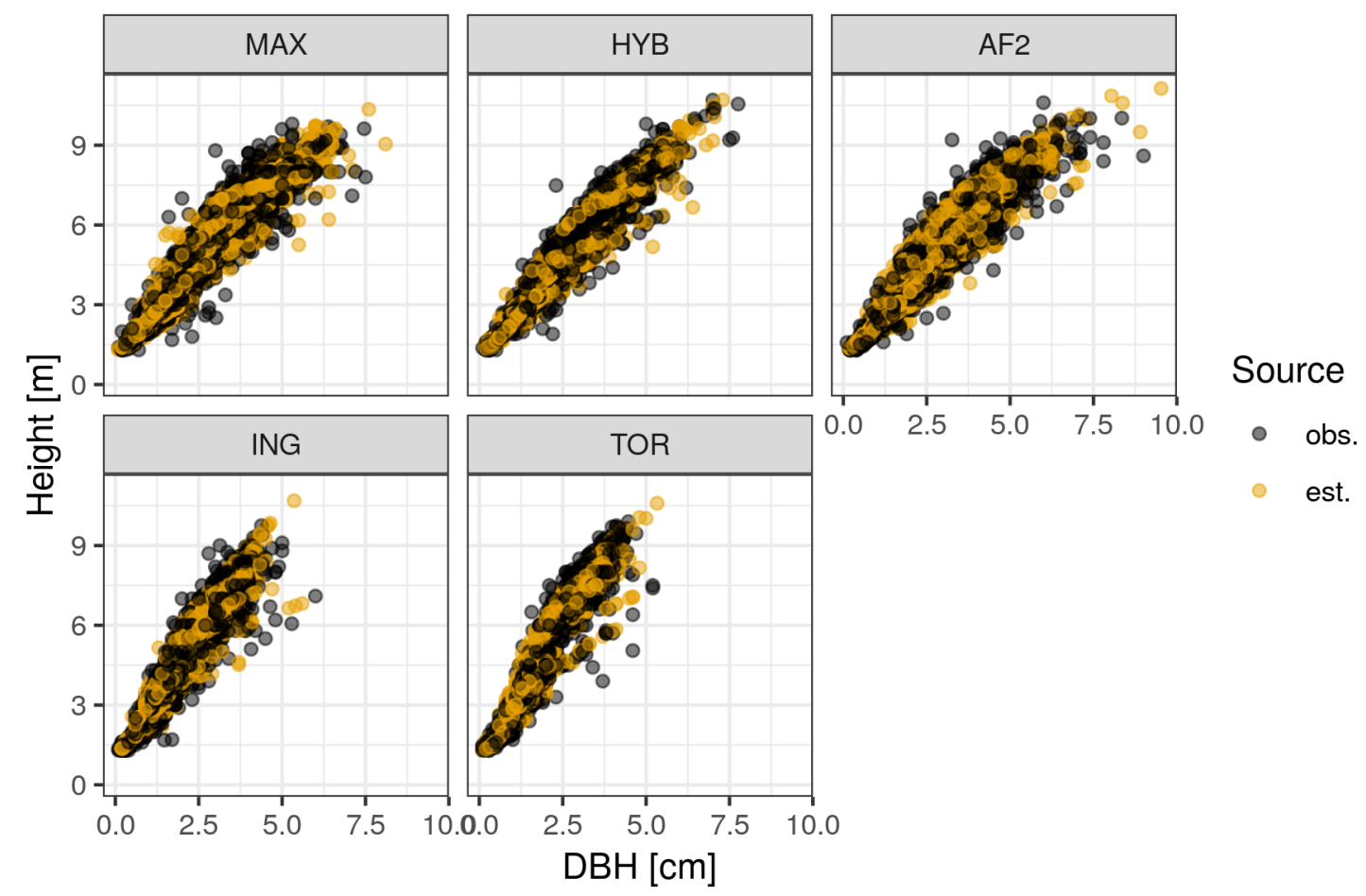

Figure 2.7: Relationship of tree height as maximum stem length and breast height diameter (DBH) during the second rotation separated by clone. Shown are the observed (obs., black points) and estimated height values (est., based on DBH, yellow points).

Again, the conformity of measured and estimated height values can be assessed as good. Analogous to the RCD, the poplars produced higher values for thickest stems than the willows. The curve progression seems again more steep for the willow stands than for poplar.

\subsubsection{First Rotation}

\section{Survival in First Vegetation Period}

Before generating single tree heights, the first step of the growth simulation is to estimate how many trees are alive and have actually grown from the cuttings after planting. A generalized linear model (diagnostics see Table 2.9) was fitted for this purpose using the quasibinomial model family with the binary coded response differentiating between alive (success: 1 ) and dead (failure: 0 ) trees. After the 
process of variable selection, 4 variables were pointed out as having a significant effect on this response. These include the mean sum of precipitation in May and June prec, the mean temperature in June and July temp, the ordinal date (day of year) of planting doyPlant and clonal affiliation clone. The available water capacity was not kept in the model due to its coefficient being non-significant.

Table 2.9: Summary of GLM fit for estimating $\eta_{\text {survInit }}$ of the probability $p_{\text {survInit }}$ of single trees having survived during the first vegetation period after planting. Variable prec resembles the mean sum of precipitation of months May and June, temp stands for the mean temperature in June and July, doyPlant represents the ordinal date of planting and clone contains the genotype. The parameterized model function is given in Equation 2.15.

\begin{tabular}{cccrcrccc}
\hline Model (link) & $\Phi$ & $D_{\text {Null }}$ & $d f_{\text {Null }}$ & $D_{\text {Model }}$ & $d f_{\text {Model }}$ & $F$ & $p$ & $D_{\text {adj }}^{2}$ \\
\hline q.-binom. (logit) & 10.22 & 4009.7 & 295 & 2705.1 & 288 & 18.2 & $<0.001$ & 0.31 \\
\hline
\end{tabular}

\begin{tabular}{lrcrr}
\hline Covariate & \multicolumn{1}{c}{ Est. } & $s e$ & \multicolumn{1}{c}{$t$} & \multicolumn{1}{c}{$p$} \\
\hline Intercept & 17.67836 & 2.30226 & 7.7 & $<0.001$ \\
prec & 0.01168 & 0.00378 & 3.1 & 0.002 \\
temp & -0.57383 & 0.09262 & -6.2 & $<0.001$ \\
doyPlant & -0.05826 & 0.00866 & -6.7 & $<0.001$ \\
cloneHYB & -0.67449 & 0.17682 & -3.8 & $<0.001$ \\
cloneAF2 & -0.05921 & 0.17396 & -0.3 & 0.734 \\
cloneING & 0.67689 & 0.20420 & 3.3 & 0.001 \\
cloneTOR & 0.46126 & 0.20019 & 2.3 & 0.022 \\
\hline
\end{tabular}

$$
\begin{aligned}
\widehat{\eta_{\text {survInit }}}=\log \left(\frac{\widehat{p_{\text {survInit }}}}{1-p_{\text {survInit }}}\right)=17.67836+0.01168 * \text { prec }-0.57383 * \text { temp } \\
\\
-0.05826 * \text { doyPlant }+\left(\begin{array}{c}
0 \\
-0.67449 \\
-0.05921 \\
0.67689 \\
0.46126
\end{array}\right) * \text { clone }
\end{aligned}
$$

Apart from that, the coefficients of the other variables all imply biologically plau- 
sible relations. While a higher sum of precipitation positively influences the survival rate, higher temperatures and a later planting date have an antagonistic effect. The coefficients for the contrast levels of the factor clone indicate that 'Hybride 275 ' had a significantly lower survival rate than 'Max 1 ' in the first year after trial establishment. Although the plots that were affected by poor planting material quality for 'Hybride 275' were removed from the data set, a negative effect is still present. It can't be fully clarified if some of this effect is still attributed to problems with the planting material which had not been documented or if it is inherent for clone 'Hybride 275'. The, in comparison, slightly less poor performance of 'AF2' is in opposite to 'Hybride 275' not significant. Of further interest is that both willow clones performed significantly better than 'Max 1'. It should also be noted that no interaction between the clonal factor and any environmental variable had a significant impact on the response. Testing for the goodness of fit, the described model is significantly better than the null model. There is considerable overdispersion present which was accounted for by the quasibinomial approach but still has a questionable magnitude of $\Phi=10.22$. Furthermore, the discrepance between the residual deviance and the residual degrees of freedom (2705.1 to 288 ) is quite large and points towards an ill fitting model (Faraway, 2016). This could not be further improved with the environmental variables at hand. Even with location as a dummy variable added to the model these effects still existed and were only slightly reduced in magnitude. Therefore the model was kept as presented here. With $D_{a d j}^{2}=0.31$ the predictive power is intermediate.

\section{Growth in First Vegetation Period}

\section{Estimation of Stand Mean Height after Planting}

After estimating the survival rate, the single tree growth during the establishment phase needs to be predicted. For generating single tree heights after the first vegetation period, the mean stand height has to be estimated first. The stepwise variable selection procedure indicated the available water capacity, the precipitation in May and June, the ordinal date of planting as day of year and clonal affiliation as having a significant influence on the response (see Table 2.10 for model diagnostics). Both available water capacity and precipitation have a positive influence, as expected. The ordinal date of planting has a negative coefficient 
which implies lower mean stand heights when planting is carried out later which is also comprehensible. These results match with the findings for the survival rate. Regarding the differences between clones it should be pointed out that the differences between 'Max 1' as the base level of the contrast and the two other poplar clones are not significant while they are for the two willow clones which reached higher mean stand heights. Again, no interaction of the factor clone with any environmental variable proved to be significant.

The predictive power of the model is satisfactory as indicated by a value of 0.55 for $R_{a d j}^{2}$.

Table 2.10: Summary of linear model fit for estimating the mean stand height lMean at the end of the first vegetation period after planting. The response was square root transformed. Independent variables are the available water capacity $a w c$, the mean sum of precipitation of months May and June prec, the ordinal date of planting doy Plant and clonal affiliation clone. The parameterized model function is given in Equation 2.16.

\begin{tabular}{ccrrrrr}
\hline$\hat{\sigma}$ & $R S S_{\text {Null }}$ & $d f_{\text {Null }}$ & $R S S_{\text {Model }}$ & $d f_{\text {Model }}$ & \multicolumn{1}{c}{$F$} & \multicolumn{1}{c}{$p$} \\
\hline 0.26 & 48.1 & 315 & 21.2 & 308 & 55.6 & $<0.001$ \\
\hline & & & & & \\
\cline { 2 - 7 } & Covariate & \multicolumn{1}{c}{ Est. } & \multicolumn{2}{c}{$s e$} & \multicolumn{1}{c}{$t$} & \multicolumn{1}{c}{$p$} \\
\cline { 2 - 7 } & Intercept & 1.40140 & 0.27284 & 5.14 & $<0.001$ \\
& awc & 0.01382 & 0.00100 & 13.81 & $<0.001$ \\
& prec & 0.00758 & 0.00077 & 9.78 & $<0.001$ \\
& doyPlant & -0.01960 & 0.00225 & -8.72 & $<0.001$ \\
& cloneHYB & -0.06708 & 0.04966 & -1.35 & 0.178 \\
& cloneAF2 & 0.03828 & 0.04502 & 0.85 & 0.396 \\
& cloneING & 0.19824 & 0.04502 & 4.40 & $<0.001$ \\
& cloneTOR & 0.17515 & 0.04577 & 3.83 & $<0.001$ \\
\hline & & & & & &
\end{tabular}




$$
\begin{aligned}
& \sqrt{\text { lMean }}=1.40140+0.01382 * \text { awc }+0.00758 * \text { prec } \\
&-0.01960 * \text { doyPlant }+\left(\begin{array}{c}
0 \\
-0.06708 \\
0.03828 \\
0.19824 \\
0.17515
\end{array}\right) * \text { clone }
\end{aligned}
$$

\section{Estimation of Weibull Distribution Parameters}

To generate height distributions, a Weibull distribution was fit separately on the plot scale to all single tree heights as described in Section 2.1.5. The plot-wise fitting was satisfactory by visual assessment and of all 416 fits the lowest $p$-Value of the corresponding Kolomogorov-Smirnov test was 0.12 so in no case the null hypothesis that the observed values originate from a distribution that equals the Weibull distribution could be rejected. The shape $c$ and scale $b$ parameters of the fitted Weibull distributions were then examined for their correlation with mean stand height.

Figure 2.8 contains the display of one of these correlations, in this case of the mean stand height and the shape parameter $c$. A clear positive but slightly nonlinear relationship can be seen.

A linear model was fit to estimate the shape parameter $c$ by the mean stand height. The response was log transformed to accommodate for the non-linearity (as indicated by the yellow line in Figure 2.8. After variable selection, the independent variables for mean stand height $l$ Mean and clonal affiliation clone were kept in the model although the differences by clone seem small. Adding the interaction of clone and $l$ Mean did not improve the fit sufficiently. The overall predictive power of the model is good with $R_{a d j}^{2}=0.75$. The model summary is given in Table 2.11. 


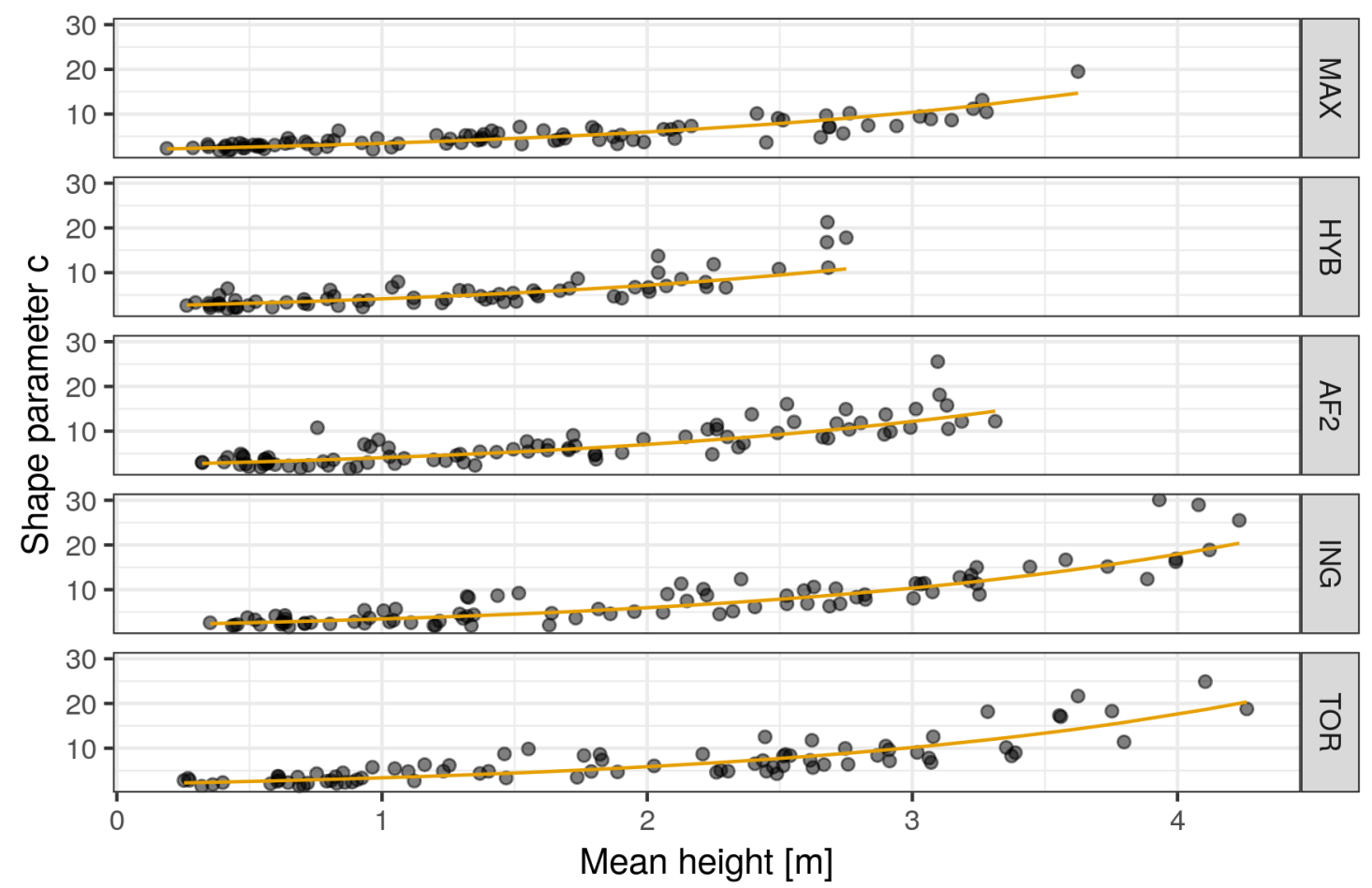

Figure 2.8: Shape parameter $c$ of each fitted Weibull distribution by mean stand height after the first vegetation period separated by clone. The yellow lines are based on the back-transformed estimations from the linear model.

Table 2.11: Summary of linear model fit for estimating the shape parameter $c$ of the Weibull distribution. The response has been log-transformed. Independent variables are the mean stand height $l$ Mean after the first vegetation period and the clonal affiliation clone.

\begin{tabular}{|c|c|c|c|c|c|c|c|}
\hline$\hat{\sigma}$ & $R S S_{\text {Null }} \quad d f$ & $l_{\text {Null }}$ & $R S S_{\text {Model }}$ & $l \quad d f_{\text {Model }}$ & $F$ & $p$ & $R_{a d j}^{2}$ \\
\hline 0.31 & 161.7 & 415 & 40.5 & 410 & 245.7 & $<0.001$ & 0.75 \\
\hline & Covariate & & Est. & se & $t$ & p & \\
\hline & Intercept & & 69183 & 0.04101 & 16.9 & $<0.001$ & \\
\hline & lMean & & .55015 & 0.01596 & 34.5 & $<0.001$ & \\
\hline & clone $H Y B$ & & 17873 & 0.05084 & 3.5 & $<0.001$ & \\
\hline & cloneAF2 & & .15763 & 0.04738 & 3.3 & $<0.001$ & \\
\hline & cloneING & & .00528 & 0.04795 & -0.1 & 0.912 & \\
\hline & cloneTOR & & .02399 & 0.04835 & -0.5 & 0.62 & \\
\hline
\end{tabular}


In Figure 2.9 the relationship of scale parameter $b$ of each fitted Weibull distribution with mean stand height can be seen. As expected, this correlation is much closer than that of the shape parameter and mean stand height.

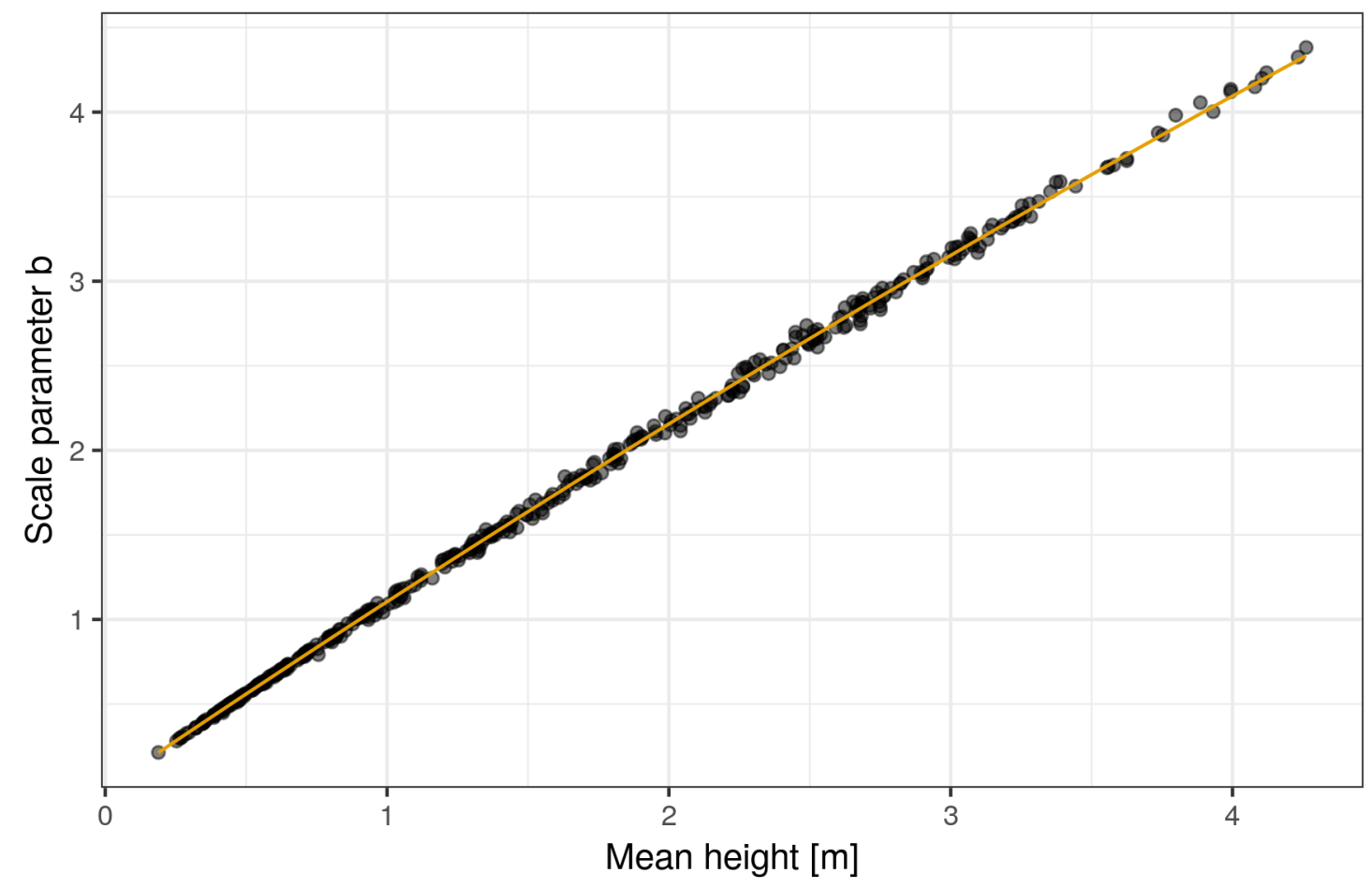

Figure 2.9: Scale parameter $b$ of each fitted Weibull distribution by mean stand height $l M e a n$ after the first vegetation period separated by clone. The yellow line is based on the estimations from the linear model using GLS.

Again, the relationship is slightly nonlinear which in this case could best be accounted for by introducing the mean stand height as a second-degree polynomial into the model. After fitting the linear model it became apparent that the variance of the residuals was nonconstant with increasing fitted values. Since no transformation of the response could adequately accommodate for this, a GLS model was parameterized with a power variance function. Because the effect for the intercept did not test to be significantly different from 0 it was left out of the model (see Table 2.12). In terms of predictive power, by comparing the observed to fitted values through $\operatorname{cor}^{2}(\hat{y}, y)=0.999$, the model performs very well.

That the scale parameter $b$ estimation has more predictive power than the shape parameter $c$ estimation was expected. 
Table 2.12: Summary of linear model fit using GLS for estimating the scale parameter $b$ of the Weibull distribution. Independent variable is the mean stand height $l$ Mean entered as a second-degree polynomial.

\begin{tabular}{|c|c|c|c|c|c|c|c|}
\hline$\hat{\sigma}$ & $\delta$ & $l l_{\text {Null }}$ & $d f_{\text {Null }}$ & $l l_{\text {Model }}$ & $d f_{\text {Model }}$ & $L R T$ & $p$ \\
\hline 0.01 & 1.04 & -601.7 & 3 & 1004.7 & 4 & 3212.9 & $<0.001$ \\
\hline & & ovariate & $\mathrm{E}$ & st. & se & $t$ & $p$ \\
\hline & & $\begin{array}{l}\text { Mean } \\
\text { Mean }\end{array}$ & $\begin{array}{r}1.1 \\
-0.0\end{array}$ & $\begin{array}{l}3222 \\
2711\end{array}$ & $\begin{array}{l}0.00154 \\
0.00080\end{array}$ & $\begin{array}{l}735.0 \\
-33.9\end{array}$ & $\begin{array}{l}<0.001 \\
<0.001\end{array}$ \\
\hline
\end{tabular}

\section{Growth in Second and Third Vegetation Period}

After establishing the plantation, the growth in the two remaining years of the first rotation has to be estimated. This refers to the height increment. As described in Section 2.1.5. additionally to the environmental variables the competition index basal area larger (BAL) is utilized here to predict height increment based on the relative dominance of a single tree. Due to the height of the trees, the basal area per tree was calculated as the sum of basal areas for each stem's RCD. In Figure 2.10 the relationship of the BAL at the end of a vegetation period with the height increment in the following vegetation period is shown. 


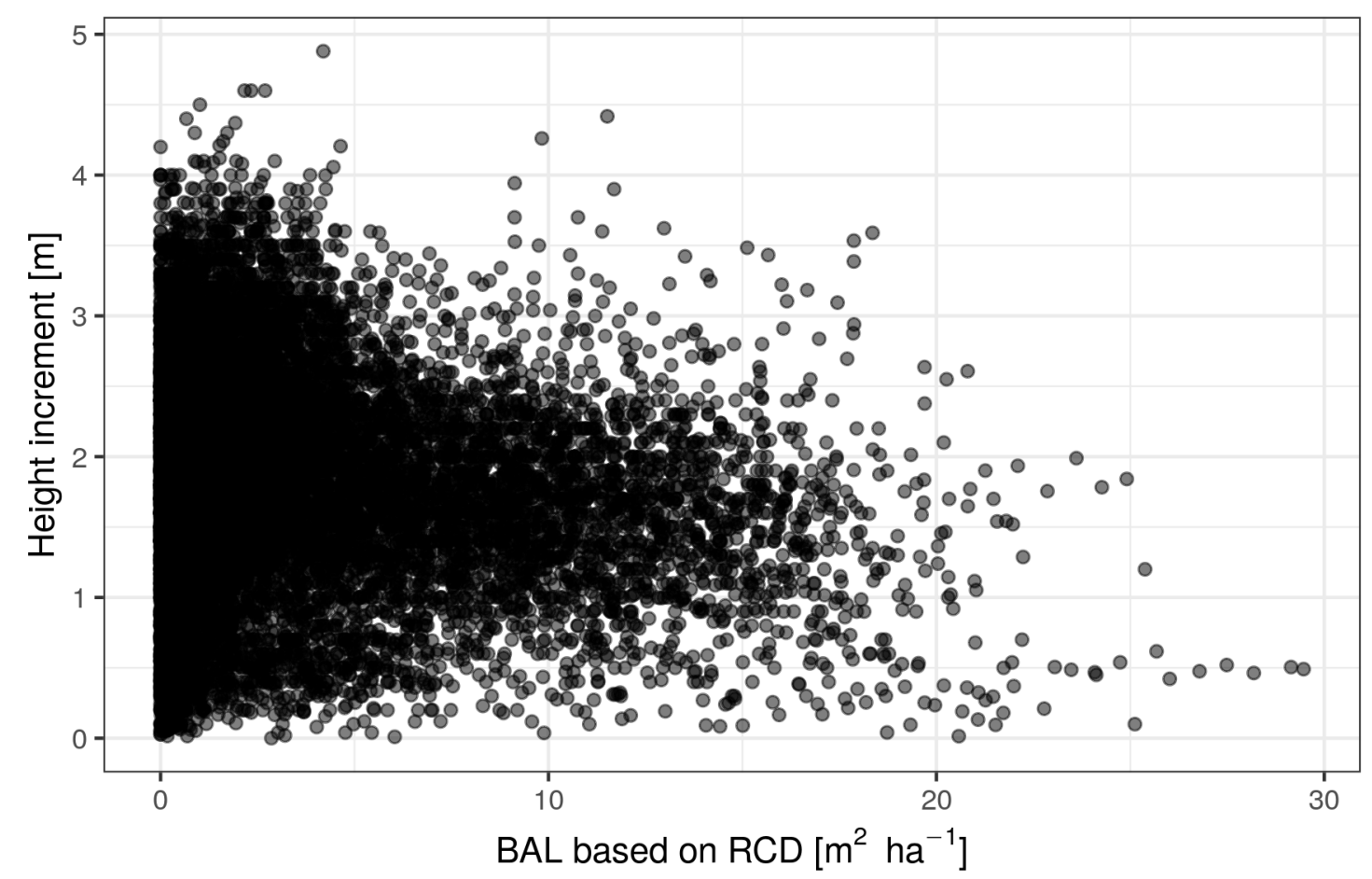

Figure 2.10: Single tree height increment by basal area larger (BAL) based on root collar diameter (RCD) during the first rotation (years 2 and 3). The height increment refers to growth of the current vegetation period while the BAL is calculated at the end of the previous growing period.

A negative trend is visible which is to be expected since an increasing BAL for a single tree implies that there are also increasingly more trees with a higher basal area and a higher relative dominance. There is still some considerable deviation present in the graph, especially for lower BAL values. This can in part be explained due to the decreasing height increment for some trees in the third year towards the end of the first rotation period. This trend is already visible in the stand height curves for RCD and height in Figure 2.6 because the slope starts to decrease with increasing RCD. The decrease in height increment with stand age combined with the clonal scale is further depicted in Figure 2.11. 


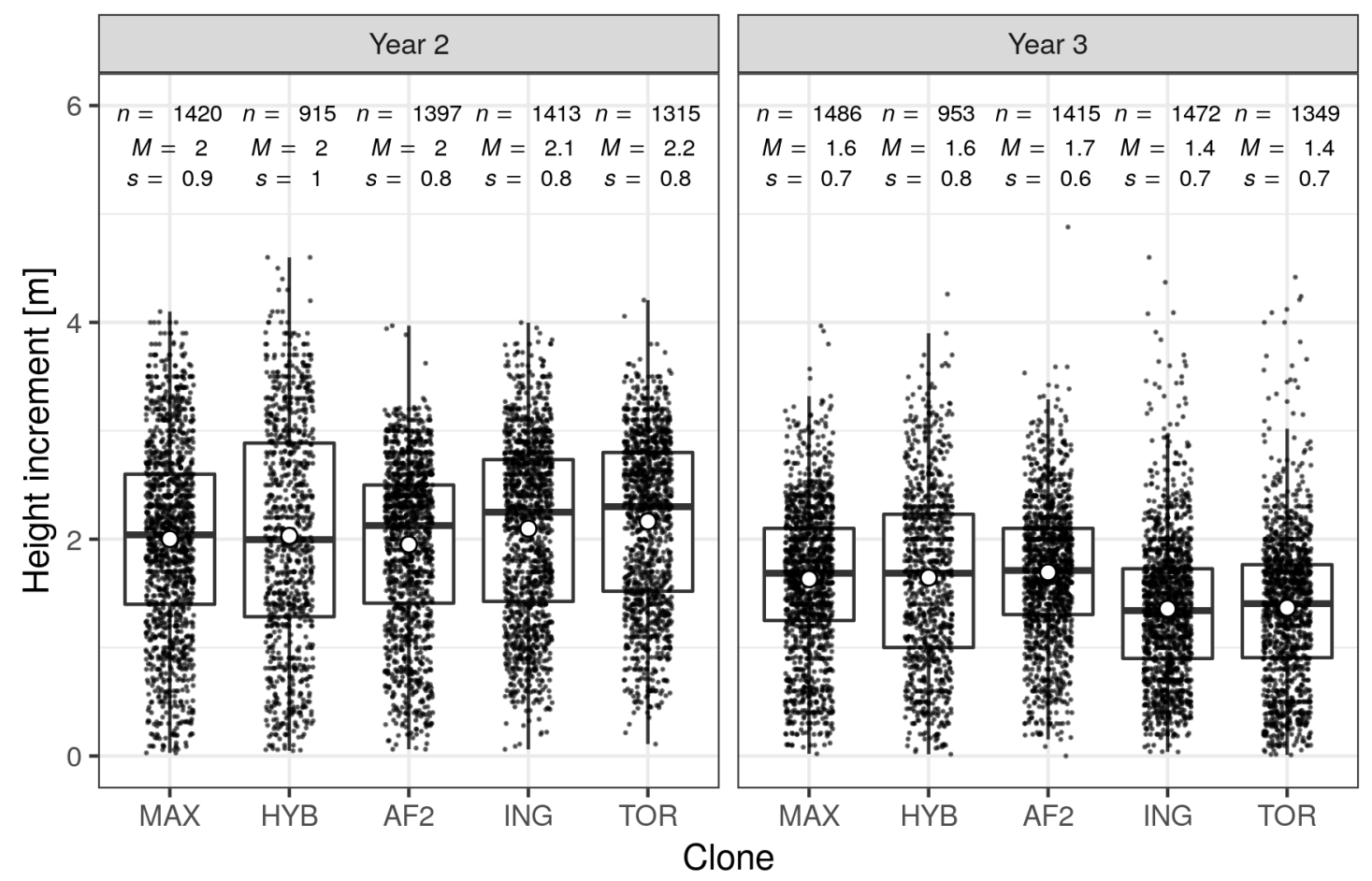

Figure 2.11: Single tree height increment by clone and separated by year during the first rotation after the plantation establishment (years 2 and 3 ).

The overall decrease from year 2 to year 3 is clearly visible. While in year 2 the clones are all on a comparable level, the willows seem to have a more pronounced decrease in the third year than the poplar clones.

Moving from descriptive to inferential statistics, a model was fitted and through variable selection the height increment determining covariates were identified. The model summary can be inferred from Table 2.13. A linear model using GLS was used here to account for the temporal correlation between years due to repeated measurement. The influence of the competition index BAL is negative as expected. For the environmental variables available water capacity $a w c$ and sum of precipitation prec at the beginning of the vegetation period have a positive influence on height increment while this is counteracted to some degree by their interaction's negative effect. The negative interaction coefficient reduces the combined influence for both variables in the higher value range and improved the fit in terms of residual structure. Increasing mean temperature in June and July has a negative effect on height increment. The already established decreasing height 
growth with increasing stand age (year) is reproduced by the model with a negative coefficient. Regarding clonal differences, the intercept is positively shifted for the willow clones in relation to 'Max 1' and the other poplar clones. Between the poplars only 'AF2' differs significantly from 'Max 1' with a negative coefficient although the magnitude of the difference is not as pronounced as for the willow clones. These effects are antagonized to some degree by the clonal interaction with stand age (year). While the negative coefficient for year is significantly more positive for 'AF2' than for 'Max 1', both willow clones have a significantly sharper decrease which corresponds with the observations made in Figure 2.11.

The overall predictive power of the height increment model for the first rotation is intermediate with $\operatorname{cor}^{2}(\hat{y}, y)=0.42$. 
Table 2.13: Summary of linear model fit using GLS for estimating the height increment during the first rotation incr LRot1. The response was square root transformed. Independent variables are the basal area larger based on RCD from the prior year balRCDPrY, the available water capacity $a w c$, the mean sum of precipitation during May and June $p r e c$, the temperature in June and July temp, the stand and tree age year and clonal affiliation clone. The colon sign stands for the interaction of two variables. The parameterized model function is given in Equation 2.17.

\begin{tabular}{cccrrrrrr}
\hline$\hat{\sigma}$ & $\hat{\Phi}$ & $l l_{\text {Null }}$ & $d f_{\text {Null }}$ & $l l_{\text {Model }}$ & $d f_{\text {Model }}$ & $L R T$ & $p$ & $\operatorname{cor}^{2}(\hat{y}, y)$ \\
\hline 0.25 & 0.14 & -4079.5 & 3 & -589.8 & 17 & 6979.5 & $<0.001$ & 0.42 \\
\hline
\end{tabular}

\begin{tabular}{lrcrc}
\hline \multicolumn{1}{c}{ Covariate } & \multicolumn{1}{c}{ Est. } & se & \multicolumn{1}{c}{$t$} & \multicolumn{1}{c}{$p$} \\
\hline Intercept & -2.41911 & 0.09823 & -24.6 & $<0.001$ \\
balRCDPrY & -0.01482 & 0.00066 & -22.5 & $<0.001$ \\
awc & 0.05136 & 0.00096 & 53.7 & $<0.001$ \\
prec & 0.03476 & 0.00059 & 58.7 & $<0.001$ \\
temp & -0.08366 & 0.00319 & -26.2 & $<0.001$ \\
year & -0.05726 & 0.00947 & -6.0 & $<0.001$ \\
cloneHYB & 0.03379 & 0.03736 & 0.9 & 0.366 \\
cloneAF2 & -0.07378 & 0.03326 & -2.2 & 0.027 \\
cloneING & 0.35765 & 0.03309 & 10.8 & $<0.001$ \\
cloneTOR & 0.46908 & 0.03374 & 13.9 & $<0.001$ \\
awc : prec & -0.00032 & 0.00001 & -48.1 & $<0.001$ \\
year : cloneHYB & -0.00645 & 0.01455 & -0.4 & 0.657 \\
year : cloneAF2 & 0.03612 & 0.01297 & 2.8 & 0.005 \\
year : cloneING & -0.15297 & 0.01289 & -11.9 & $<0.001$ \\
year $:$ cloneTOR & -0.19720 & 0.01315 & -15.0 & $<0.001$ \\
\hline
\end{tabular}




$$
\begin{aligned}
\sqrt{\text { incrLRot } 1}= & -2.41911-0.01482 * \text { balRCDPrY } Y+0.05136 * \text { awc } \\
& +0.03476 * \text { prec }-0.08366 * \text { temp } \\
& +\left(-0.05726+\left(\begin{array}{c}
0 \\
-0.00645 \\
0.03612 \\
-0.15297 \\
-0.19720
\end{array}\right) * \text { clone }\right) * \text { year }+\left(\begin{array}{c}
0 \\
0.03379 \\
-0.07378 \\
0.35765 \\
0.46908
\end{array}\right) * \text { clone } \\
& -0.00032 * \text { awc } * \text { prec }
\end{aligned}
$$

\section{Data Completion During First Rotation}

Since it is needed for calculating the BAL, the basal area per tree has to be estimated using the height. In Figure 2.12 the relationship of both parameters is displayed within the first rotation separated by clone.

Both variables are clearly positively correlated although the relationship is clearly nonlinear. Therefore a square root transformation was applied to the response which improved the residual structure to being normally distributed. Because an increase in variance with increasing fitted values was still visible a GLS model was fitted allowing for an increasing variance through the power variance function varPower. Because all years from the first rotation were included, which implies repeated measurements of the same trees, the corAR1 function was used to accommodate for the temporal correlation structure within the data. For both parameters the estimates are given in Table 2.14. The predictive power can be evaluated as good with a $\operatorname{cor}^{2}(\hat{y}, y)=0.86$. Through the variable selection procedure the variables height as length $l$ of the tallest stem, clone and their interaction were identified as having significant effects. Assessing the clonal differences in Figure 2.12, the differences do not seem to be too pronounced though. 


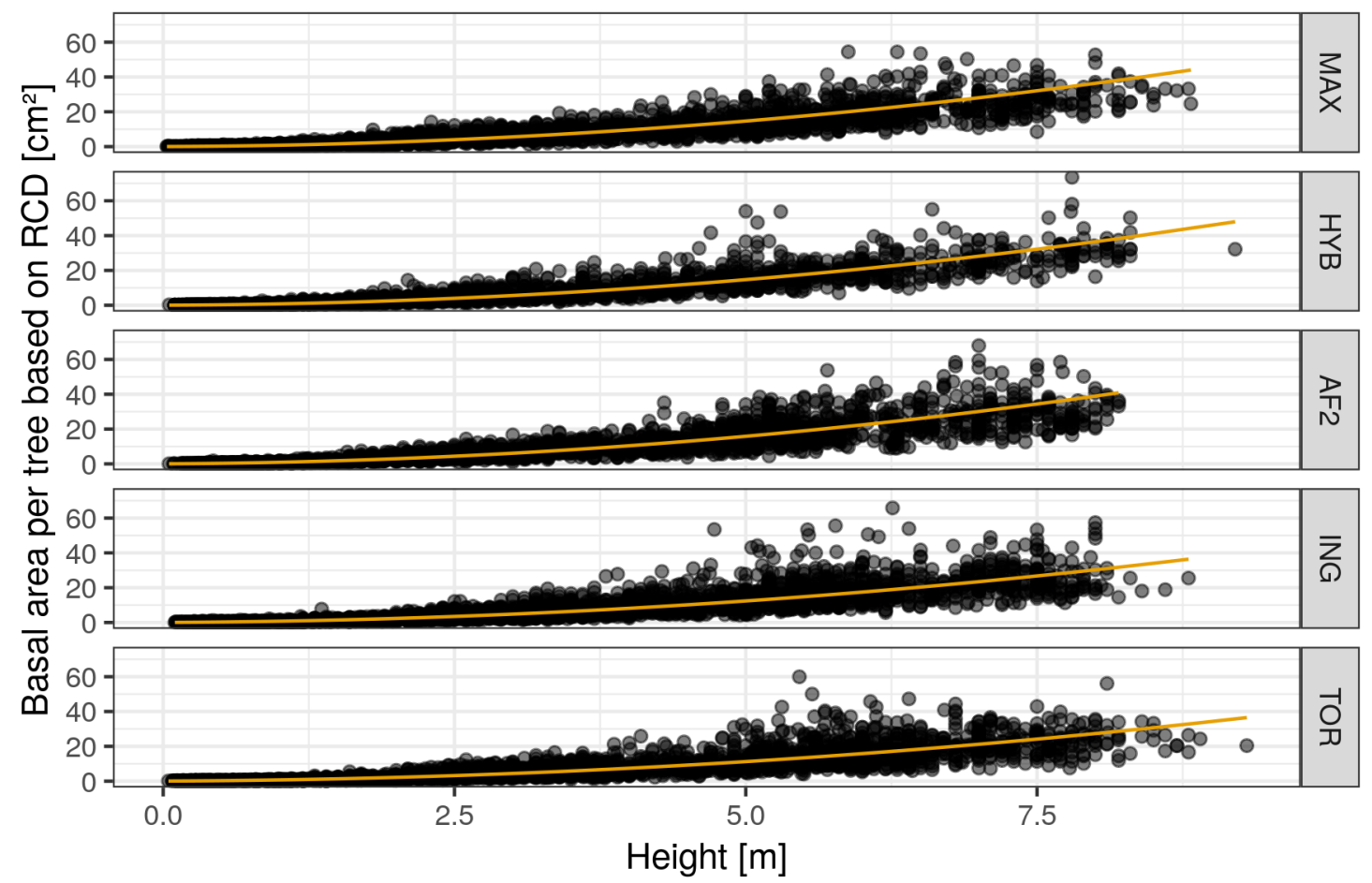

Figure 2.12: Relationship between tree-wise basal area calculated as the sum of single stem root collar diameter (RCD) basal area and height as the length of the tallest stem per tree. The data is displayed separately by clone and only value pairs from the first rotation are presented here. The yellow lines resemble the back-transformed response from the fitted model. 
Table 2.14: Summary of linear model fit using GLS for estimating the tree-wise basal area $r c d B a s$ calculated as the sum of single stem root collar diameter $(\mathrm{RCD})$ basal area. Independent variables are the tree height as the length of the tallest stem $l$ and clonal affiliation clone. The response has been square root transformed. The colon sign stands for the interaction of two variables. The parameterized model function is given in Equation 2.18.

\begin{tabular}{|c|c|c|c|c|c|c|c|c|c|}
\hline$\hat{\sigma}$ & $\hat{\Phi}$ & $\hat{\delta}$ & $l l_{\text {Null }}$ & $d f_{\text {Null }}$ & \multicolumn{2}{|c|}{$l l_{\text {Model }}$} & $d f_{\text {Model }}$ & $L R T$ & $p$ \\
\hline \multirow[t]{12}{*}{0.26} & 1.33 & 0.80 & -23614.3 & 4 & \multicolumn{2}{|c|}{-6376.8} & 13 & 34475. & $0<0.001$ \\
\hline & & \multicolumn{2}{|c|}{ Covariate } & \multicolumn{2}{|c|}{ Est. } & \multicolumn{2}{|c|}{ se } & $t$ & $p$ \\
\hline & & \multicolumn{2}{|c|}{ Intercept } & \multicolumn{2}{|c|}{0.16448} & \multicolumn{2}{|c|}{0.00631} & 26.1 & $<0.001$ \\
\hline & & \multicolumn{2}{|c|}{$l$} & \multicolumn{2}{|c|}{0.73343} & \multicolumn{2}{|c|}{0.00502} & 146.2 & $<0.001$ \\
\hline & & \multicolumn{2}{|c|}{ clone $H Y B$} & \multicolumn{2}{|c|}{-0.00493} & \multicolumn{2}{|c|}{0.00967} & -0.5 & 0.61 \\
\hline & & \multicolumn{2}{|c|}{ clone AF2 } & \multicolumn{2}{|c|}{0.05258} & \multicolumn{2}{|c|}{0.01010} & 5.2 & $<0.001$ \\
\hline & & \multicolumn{2}{|c|}{ cloneING } & \multicolumn{2}{|c|}{0.05113} & \multicolumn{2}{|c|}{0.00997} & 5.1 & $<0.001$ \\
\hline & & \multicolumn{2}{|c|}{ cloneTOR } & \multicolumn{2}{|c|}{0.05948} & \multicolumn{2}{|c|}{0.00940} & 6.3 & $<0.001$ \\
\hline & & \multicolumn{2}{|c|}{$l:$ clone $H Y B$} & \multicolumn{2}{|c|}{0.00207} & \multicolumn{2}{|c|}{0.00766} & 0.3 & 0.787 \\
\hline & & \multicolumn{2}{|c|}{$l:$ clone $A F 2$} & \multicolumn{2}{|c|}{0.01959} & \multicolumn{2}{|c|}{0.00727} & 2.7 & 0.007 \\
\hline & & \multicolumn{2}{|c|}{$l:$ clone ING } & \multicolumn{2}{|c|}{-0.07241} & \multicolumn{2}{|c|}{0.00688} & -10.5 & $<0.001$ \\
\hline & & \multicolumn{2}{|c|}{$l:$ cloneTOR } & \multicolumn{2}{|c|}{-0.10775} & \multicolumn{2}{|c|}{0.00677} & -15.9 & $<0.001$ \\
\hline
\end{tabular}

$\widehat{\sqrt{r c d B a s}}=0.16448+\left(0.73343+\left(\begin{array}{c}0 \\ 0.00207 \\ 0.01959 \\ -0.07241 \\ -0.10775\end{array}\right) *\right.$ clone $) * l+\left(\begin{array}{c}0 \\ -0.00493 \\ 0.05258 \\ 0.05113 \\ 0.05948\end{array}\right) *$ clone 
With the end of the first rotation cycle, estimating the mean annual dry mass increment becomes necessary. As described, this was implemented using the mean stand height. Figure 2.13 presents the relationship of both variables separated by clone. Similar to tree height and RCD basal area, the correlation is positive but clearly nonlinear.

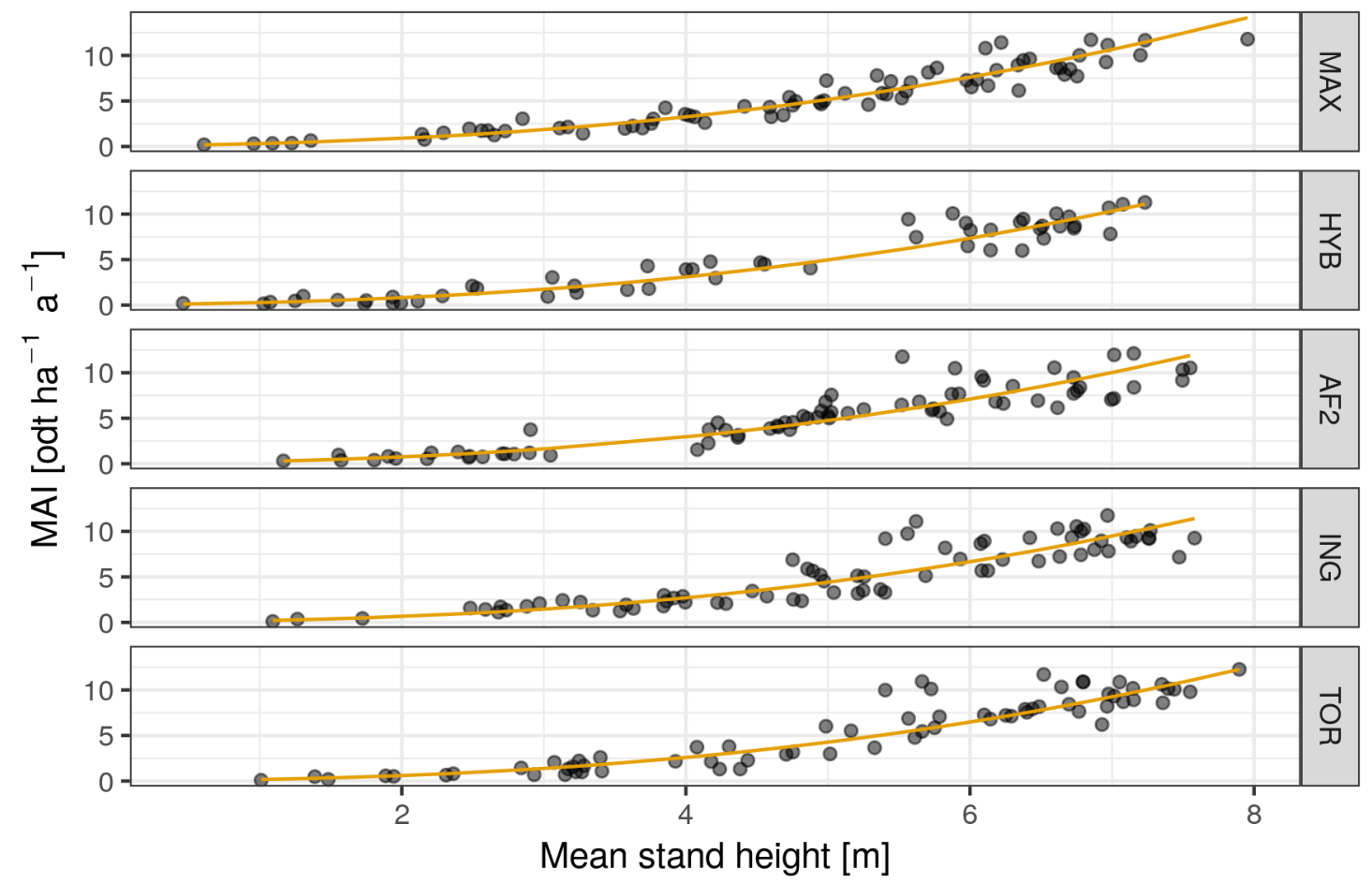

Figure 2.13: Relationship between mean stand height and mean annual increment at the end of the third vegetation period which is the end of the first rotation cycle. The data is displayed separately by clone. The yellow lines resemble the back-transformed response from the fitted model.

The differences in these relationships between clones do not seem to be too distinct. The variable selection pointed towards keeping the clonal factor within the corresponding model, while the coefficients resemble the visual assessment. There is some trend though within the poplars for an increasing negative coefficient going from 'Max 1' to 'AF2' which further increases for the two willow clones. The overall predictive power of the model can be seen as good with $R_{a d j}^{2}=0.9$. The response had to be transformed by raising it to a power of 0.4 which was estimated with the Box-Cox procedure. The model summary is given in Table 2.15 . 
Table 2.15: Summary of linear model fit for estimating the mean annual increment MAI during the first rotation based on dry mass after the third vegetation period. The response was transformed as $M A I^{0.4}$. Independent variables are the mean stand height $l M e a n$ after the third vegetation period and the clonal affiliation clone.

\begin{tabular}{|c|c|c|c|c|c|c|c|}
\hline$\hat{\sigma}$ & $R S S_{\text {Null }}$ & $d f_{N u}$ & $R S S_{\text {Mode }}$ & $d f_{\text {Model }}$ & $F$ & $p$ & $R_{a d j}^{2}$ \\
\hline \multirow[t]{8}{*}{0.19} & 124.8 & 33 & 11. & 330 & 635.5 & $<0.001$ & 0.90 \\
\hline & Cova & ate & Est. & se & $t$ & $p$ & \\
\hline & Inter & & 0.30820 & 0.03515 & 8.8 & $<0.001$ & \\
\hline & lMea & & 0.32405 & 0.00577 & 56.2 & $<0.001$ & \\
\hline & clone & $Y B$ & -0.03229 & 0.03439 & -0.9 & 0.349 & \\
\hline & clone. & & -0.06225 & 0.03145 & -2.0 & 0.049 & \\
\hline & clone & & -0.11654 & 0.03151 & -3.7 & $<0.001$ & \\
\hline & cloné & $O R$ & -0.14117 & 0.03198 & -4.4 & $<0.001$ & \\
\hline
\end{tabular}

\subsubsection{Second Rotation}

After the first rotation is completed in the third year, all biomass is being harvested by cutting the trees. The resprouting occurs in year 4 which necessitates estimating the survival and tree growth after cutting. Afterwards, the growth in the fifth and sixth year are estimated. Before continuing to describe the modeling procedure it should be kept in mind that due to administrative changes within the joint research project ProLoc and uncertainties in the continuation of the project not all trial sites were measured in the fourth year after resprouting. The stands with available data which were used for the modeling procedure presented here (excluding stands with weed influence, moors and post mining sites) are listed in Table 2.16. 
Table 2.16: ProLoc trial sites that were measured in trial year 4 after cutting.

\begin{tabular}{rll}
\hline VFL-Nr. & \multicolumn{1}{c}{ Trial site } & \multicolumn{1}{c}{ Supervising institution } \\
\hline pl01 & Emmendingen & FVA Baden-Württemberg \\
pl04 & Forchheim & LTZ Augustenberg \\
pl06 & Kupferzell & LTZ Augustenberg \\
pl12 & Kummerow & HNE Eberswalde (FH) \\
pl17 & Unterrieden & Universität Kassel \\
pl19 & Werlte & 3N Kompetenzzentrum Niedersachsen \\
pl20 & Borlinghausen & MR Höxter-Warburg \\
pl28 & Bernburg & LLFG Sachsen-Anhalt \\
pl29 & Hayn & LLFG Sachsen-Anhalt \\
pl30 & Iden & LLFG Sachsen-Anhalt \\
pl31 & Pommritz & LFULG Sachsen \\
pl35 & Trenthorst & TI - Fachinstitut für Forstgenetik Großhansdorf \\
pl36 & Lohberg & Baumschulen Oberdorla GmbH \\
pl37 & Dornburg & Thüringer Landesanstalt für Landwirtschaft \\
\hline
\end{tabular}

\section{Survival After Resprout}

Although followed mostly by vigorous growth if the tree survives, removing the above-ground biomass when harvesting imposes considerable stress on the tree and its root stock, especially if applied repeatedly (Sennerby-Forsse et al., 1992).

Figure 2.14 shows the relationship of tree height before cutting and survival after cutting. The response of the modeling approach here is the binary variable alive (success: 1) or dead (failure: 0 ) and within these two options the distribution of tree height before cutting is plotted.

First, it should be noted that the number of trees that were alive before cutting and then died is much lower than the number of trees that survived the harvest ( $n=4051$ versus $n=452$ ). Apart from that, the trees that died were tending to be smaller in height than those that survived, although there is still some considerable variation. 


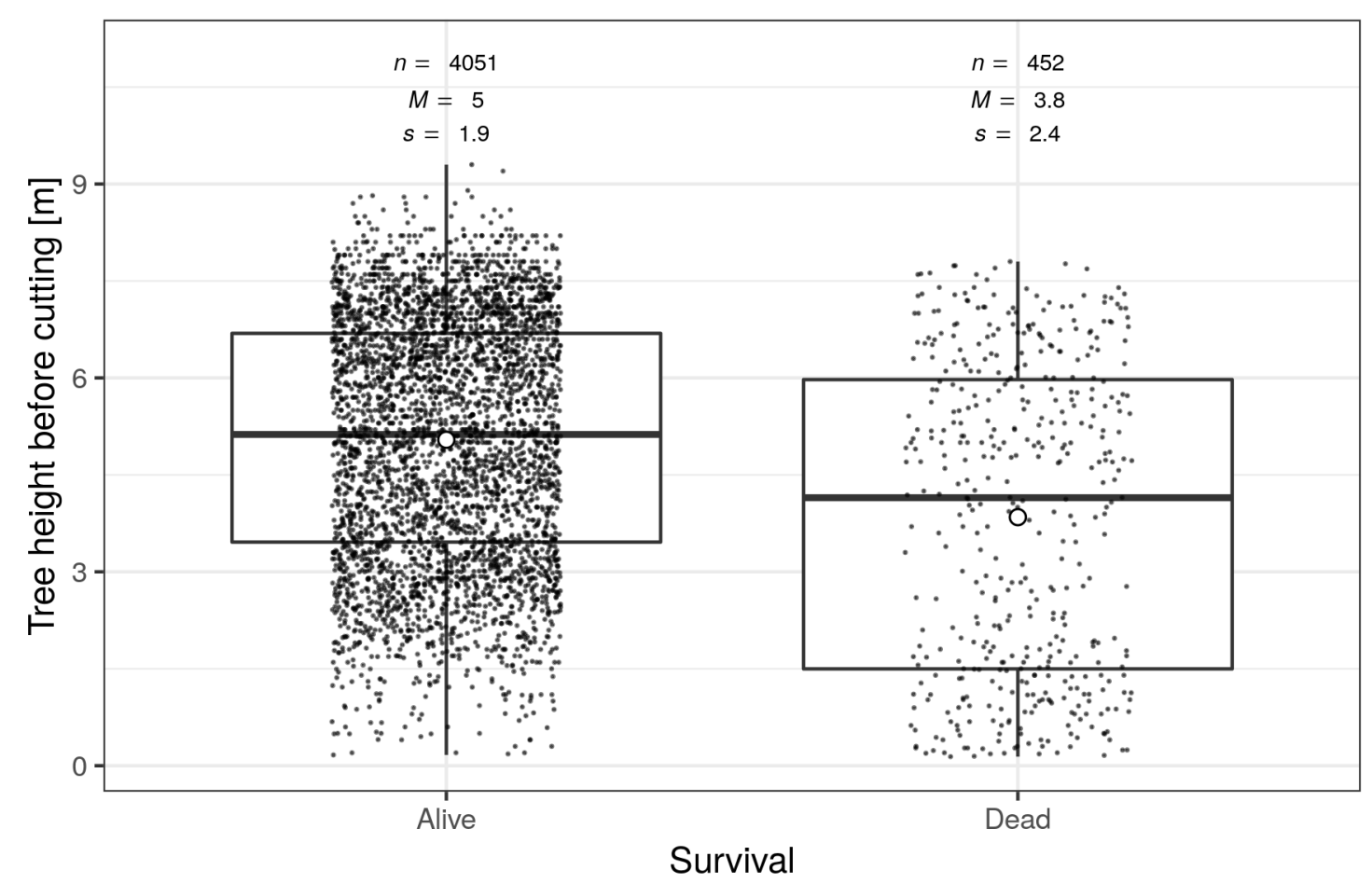

Figure 2.14: Relationship of height as the length of the tallest stem per tree after the fourth vegetation period, hence after cutting, by height as the length of the tallest stem before cutting in year 3 .

This trend could be incorporated into a glm for modeling the survival rate after the first harvest (for the model summary see Table 2.17. Environmental variables that were kept in the model were the mean sum of precipitation prec in May and June and mean temperature temp in June and July. The former does not have an effect which differs significantly from 0 ( $p$-value of $t$-test is 0.366$)$. It was still kept in the model though, as indicated by the variable selection. The effects of prec and temp have the same signs as in the previous models. Examining the genotypic influence, in comparison to 'Max 1' clone 'Hybride 275' performs slightly worse although the effect is not significant to $\alpha=0.05$. Both willow clones do not significantly differ from 'Max 1' although the higher standard errors here should be noted. Trees of the clone 'AF2' on the contrary have a significantly lower probability of survival after cutting in comparison to 'Max 1'. 
Table 2.17: Summary of GLM fit for estimating $\eta_{\text {survResp }}$ of the probability $p_{\text {survResp }}$ of single trees having survived after coppicing. Variable $l$ is the height as the length of the tallest stem before harvest in year 3 , prec resembles the mean sum of precipitation of months May and June, temp stands for the mean temperature in June and July and clone contains the genotype. The parameterized model function is given in Equation 2.19 .

\begin{tabular}{cccccccc}
\hline Model (link) & $D_{\text {Null }}$ & $d f_{\text {Null }}$ & $D_{\text {Model }}$ & $d f_{\text {Model }}$ & $\chi^{2}$ & $p$ & $D_{a d j}^{2}$ \\
\hline binom. (logit) & 2935.2 & 4502 & 2628.6 & 4495 & 306.6 & $<0.001$ & 0.10 \\
\hline
\end{tabular}

\begin{tabular}{lrcrr}
\hline Covariate & \multicolumn{1}{c}{ Est. } & $s e$ & \multicolumn{1}{c}{$z$} & \multicolumn{1}{c}{$p$} \\
\hline Intercept & 15.44952 & 1.33714 & 11.6 & $<0.001$ \\
$l$ & 0.28076 & 0.03377 & 8.3 & $<0.001$ \\
prec & 0.00322 & 0.00357 & 0.9 & 0.366 \\
temp & -0.84555 & 0.08255 & -10.2 & $<0.001$ \\
cloneHYB & -0.29395 & 0.16412 & -1.8 & 0.073 \\
clone AF2 & -0.63943 & 0.14879 & -4.3 & $<0.001$ \\
cloneING & 0.27084 & 0.17499 & 1.5 & 0.122 \\
cloneTOR & -0.06500 & 0.17046 & -0.4 & 0.703 \\
\hline
\end{tabular}

$$
\begin{aligned}
\eta_{\text {survResp }}=\log \left(\frac{\widehat{p_{\text {survResp }}}}{1-p_{\text {survResp }}}\right)=15.44952+0.28076 * l+0.00322 * \text { prec } \\
\\
-0.84555 * \text { temp }+\left(\begin{array}{c}
0 \\
-0.29395 \\
-0.63943 \\
0.27084 \\
-0.06500
\end{array}\right) * \text { clone }
\end{aligned}
$$

Regarding the model performance the overall goodness of fit by comparing the fitted model to a null model is satisfactory. In opposition to the glm focusing on the survival after stand initialization, no considerable overdispersion could be detected here therefore the binomial instead of the quasibinomial model family could be used. Comparing the deviance with the degrees of freedom confirms that the model is not ill-fitting. However, the predictive power is poor as indicated by $D_{a d j}^{2}=0.1$. This could not sufficiently be improved by adding further environ- 
mental variables or it lead to implausible results.

\section{Growth After Resprout}

After determining if a tree survived the harvest, the next simulation step is to estimate the growth of the surviving trees during the fourth vegetation period. As it is the case for the survival model, the height before cutting shows some correlation with the response. Figure 2.15 showcases this positive relationship.

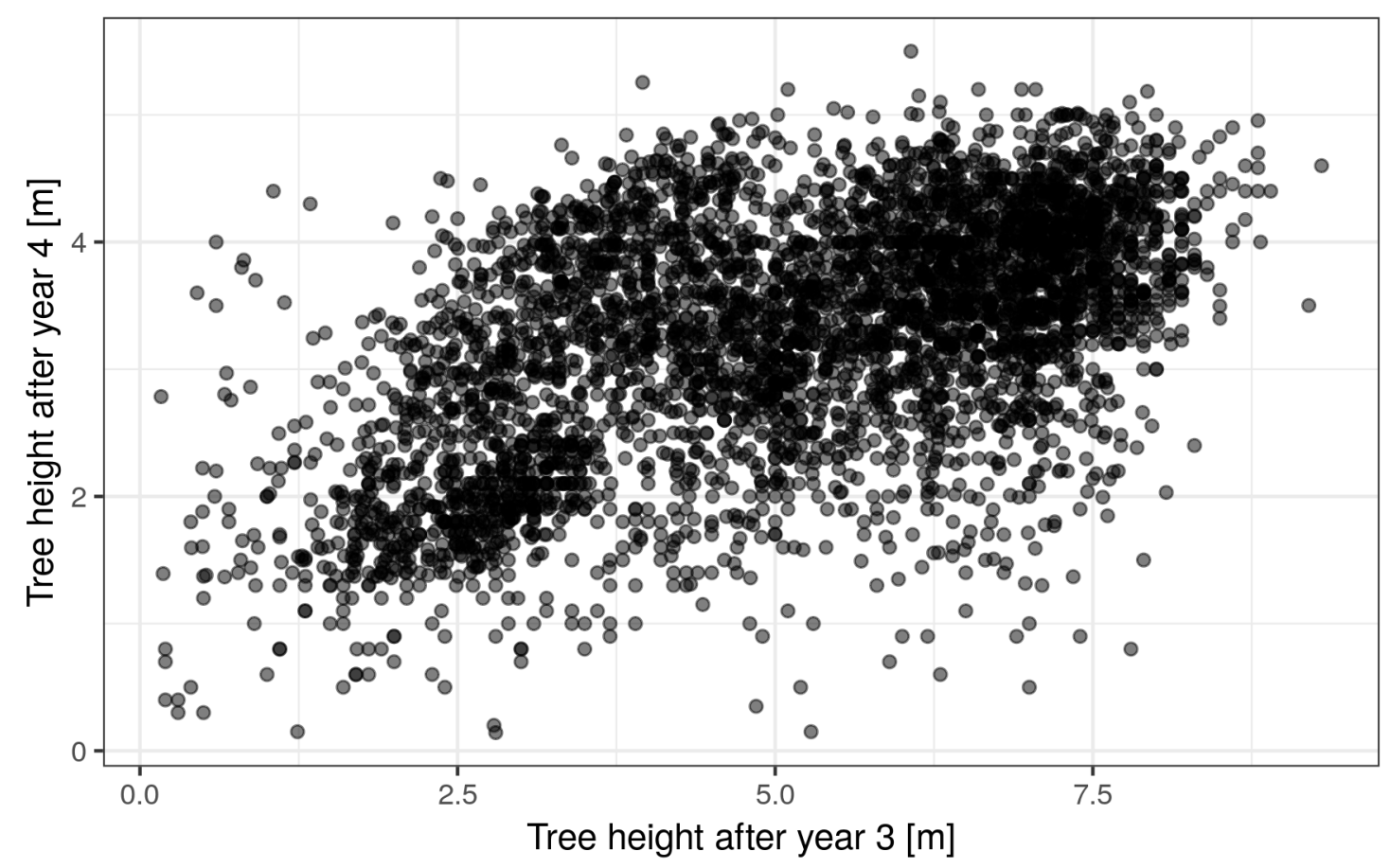

Figure 2.15: Distribution of height values as the length of the tallest stem per tree before cutting in year 3 separated by whether the tree is still alive in the fourth vegetation period after the harvest.

Table 2.18 contains the diagnostics for the model fitted to estimate height increment after resprout. The height as length before cutting has the expected positive coefficient. As environmental variables precipitation and temperature have their expected influence. Instead of the available water capacity the German agricultural soil quality rating is used here as it has an effect which differs significantly from 0 . The latter was not the case for the available water capacity and using the 
soil quality rating BZ here improved the model fit significantly. The effect is positive as expected. Clonal differentiation indicates that 'Hybride 275' and 'AF2' were less vigorous after cutting than 'Max 1'. Willow clone 'Inger' is on a comparable level while 'Tordis' performs significantly better.

The predictive power of the model is intermediate signified by $R_{a d j}^{2}=0.47$.

Table 2.18: Summary of linear model fit for estimating the height increment incr Resp after resprout during the fourth vegetation period. Independent variables are the tree height as length of the tallest stem $l$ before cutting in year 3 , the German agricultural soil quality index $B Z$, the mean sum of precipitation during May and June prec, the mean temperature in June and July temp and clonal affiliation clone. The parameterized model function is given in Equation 2.20.

\begin{tabular}{|c|c|c|c|c|c|}
\hline$\frac{\hat{\sigma}}{0.68}$ & \multirow{2}{*}{$\begin{array}{r}R S S_{\text {Null }} \\
3691.3\end{array}$} & $R S S_{\text {Model }}$ & \multirow{2}{*}{$\begin{array}{rr}l & d f_{\text {Model }} \\
& 4227\end{array}$} & \multirow{2}{*}{$\frac{F}{425.5}$} & \multirow{2}{*}{$\frac{p}{<0.001}$} \\
\hline \multirow[t]{12}{*}{0.68} & & 1936.6 & & & \\
\hline & Covariate & $\overline{\text { Est. }}$ & se & $t$ & $p$ \\
\hline & Intercept & 7.56286 & 0.32869 & 23.0 & $<0.001$ \\
\hline & $l$ & 0.15150 & 0.00773 & 19.6 & $<0.001$ \\
\hline & $B Z$ & 0.02163 & 0.00343 & 6.3 & $<0.001$ \\
\hline & prec & 0.01871 & 0.00151 & 12.4 & $<0.001$ \\
\hline & temp & -0.45487 & 0.01680 & -27.1 & $<0.001$ \\
\hline & clone $H Y B$ & -0.36805 & 0.03490 & -10.5 & $<0.001$ \\
\hline & clone $A F 2$ & -0.39262 & 0.03189 & -12.3 & $<0.001$ \\
\hline & cloneING & 0.03265 & 0.03094 & 1.1 & 0.291 \\
\hline & cloneTOR & 0.17265 & 0.03234 & 5.3 & $<0.001$ \\
\hline & $B Z:$ prec & -0.00010 & 0.00003 & -3.9 & $<0.001$ \\
\hline
\end{tabular}

incrResp $=7.56286+0.15150 * l+0.02163 * B Z+0.01871 *$ prec

$-0.45487 *$ temp $-0.00010 * B Z *$ prec $+\left(\begin{array}{c}0 \\ -0.36805 \\ -0.39262 \\ 0.03265 \\ 0.17265\end{array}\right) *$ clone 


\section{Growth During Fifth and Sixth Vegetation Period}

\section{Growth of Trees with Height $>1.3 \mathrm{~m}$}

As it was the case for height increment and BAL based on RCD during the first rotation, the $\mathrm{BAL}$ based on $\mathrm{DBH}$ has a negative effect on height increment, as can be seen in Figure 2.16 .

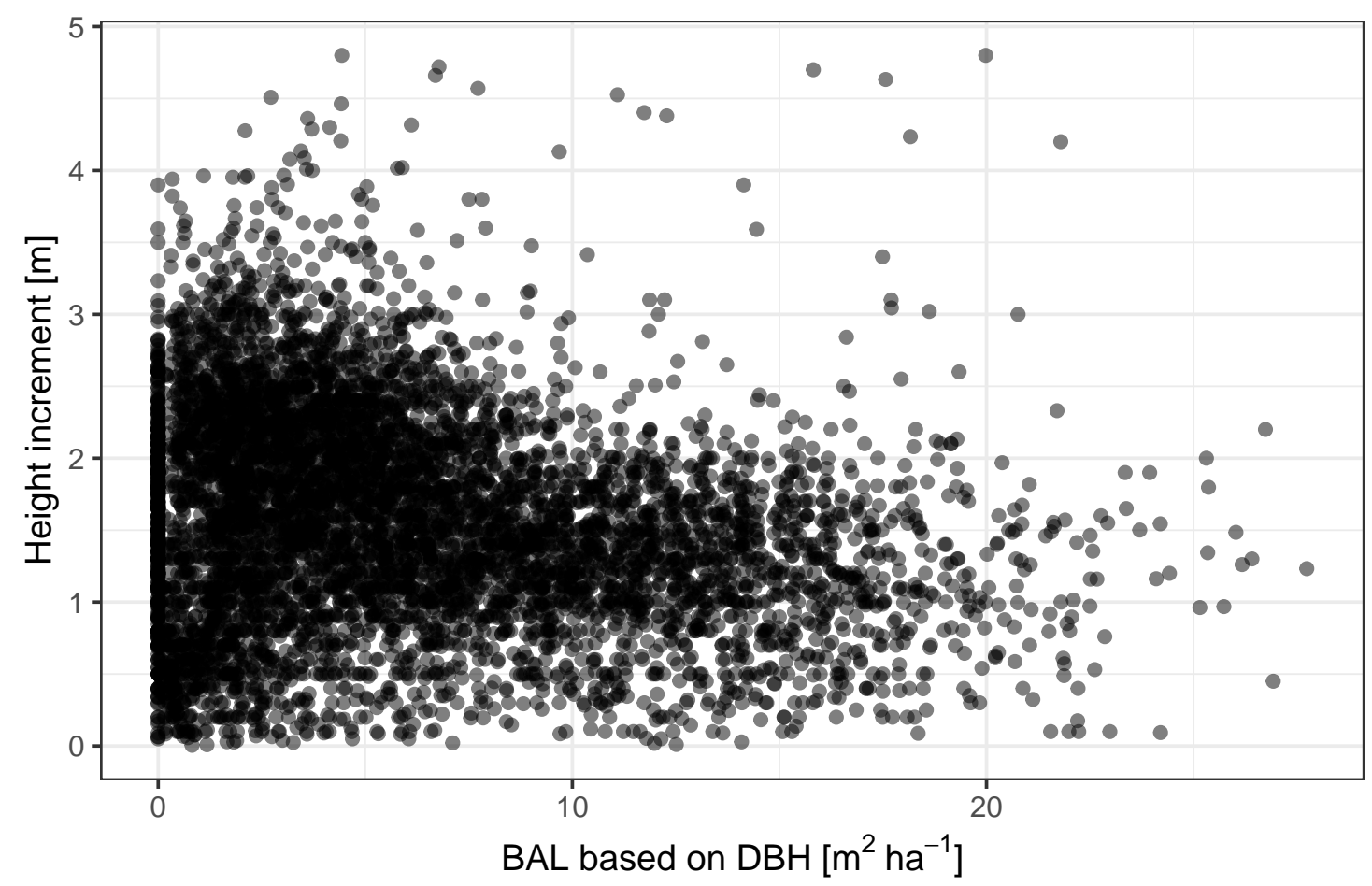

Figure 2.16: Single tree height increment by basal area larger (BAL) based on $\mathrm{DBH}$ during the second rotation (years 5 and 6). The height increment refers to growth of the current vegetation period while the BAL is calculated at the end of the previous growing period.

Again, the higher variation in height increment for lower BAL values is conspicuous. This can in part be explained by the temporal trend that height increment decreases from the fifth to the sixth stand year, comparable to the effect in the first rotation. The decrease is illustrated in Figure 2.17. 


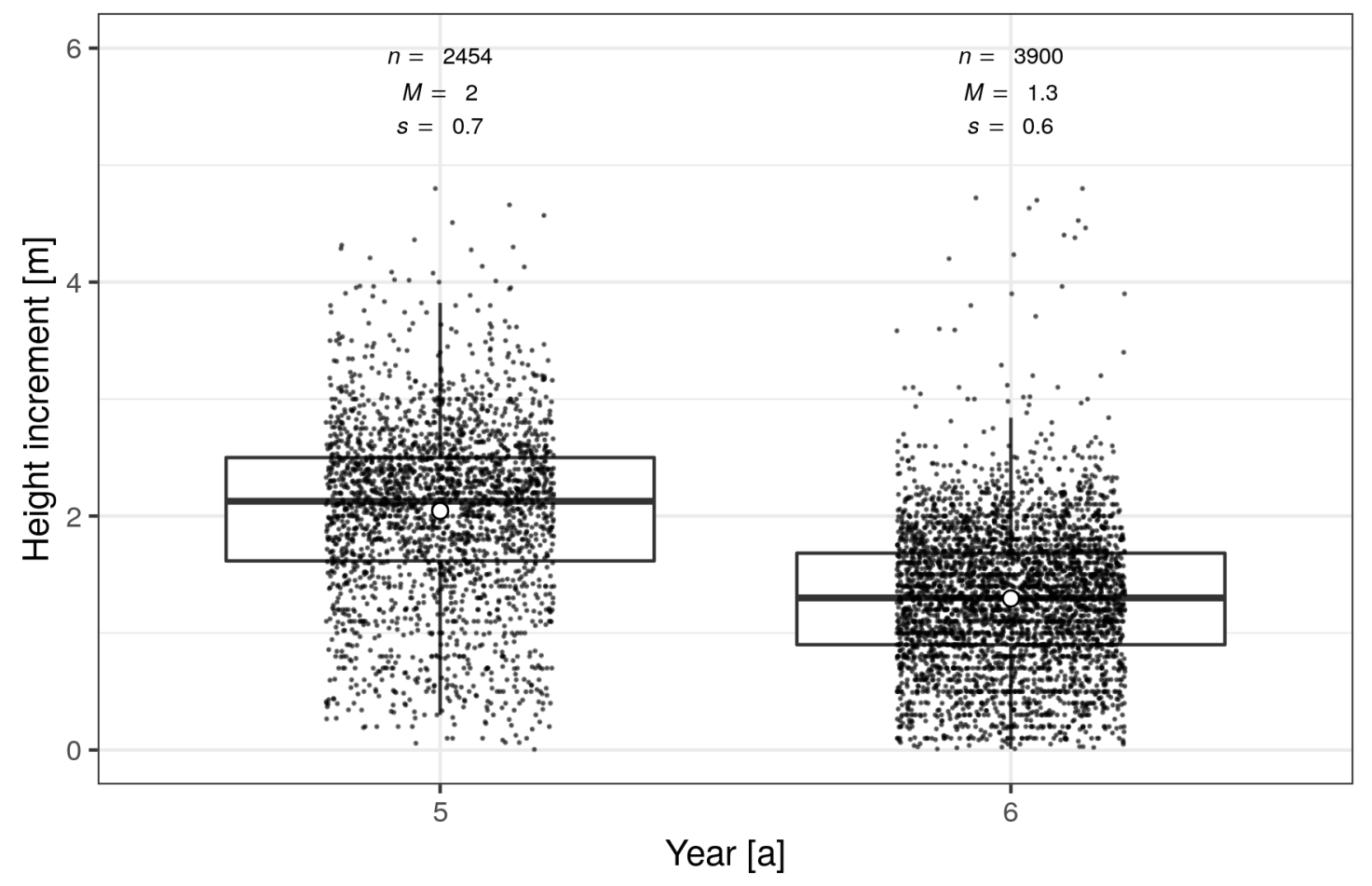

Figure 2.17: Single tree height increment by year during the second rotation after the resprouting (years 5 and 6 ).

The differing numbers of observations here originate from the fact that measurements were carried out in fewer stands in year 4, hence fewer plausible recordings were available to also calculate the increment from year 4 to year 5. For increment in year 6 the full dataset was available again.

Table 2.19 contains the diagnostics for the single tree growth model in the second rotation. As for the first rotation a linear model with GLS was fit to incorporate a correlation structure for repeated measurements and a variance power function for heteroscedasticity. Furthermore, the response was square root transformed.

The competition index balPr $Y$ and year have their expected influence which is also the case for the environmental variables soil quality rating $B Z$, mean sum of precipitation in May and June prec and mean temperature temp in June and July. Similar to the growth after coppicing using the soil quality rating instead of the available water capacity improved the fit significantly. Its interaction with precipitation negligibly improved the model fit and lead to problems with the residual structure. In contrast to the first rotation, the interaction of clone and year was not 
kept in the model due to the same reasons.

The predictive power of the model is intermediate with a squared correlation of $\operatorname{cor}^{2}(\hat{y}, y)=0.37$ between observed and estimated values.

Table 2.19: Summary of linear model fit using GLS for estimating the height increment during the second rotation incr HRot2. The response was square root transformed. Independent variables are the basal area larger based on $\mathrm{DBH}$ from the prior year balPrY, the soil quality in$\operatorname{dex} B Z$, the mean sum of precipitation during May and June prec, the mean temperature in June and July temp, the stand and tree age year and clonal affiliation clone. The parameterized model function is given in Equation 2.21

\begin{tabular}{ccccrcrcrr}
\hline$\hat{\sigma}$ & $\hat{\Phi}$ & $\hat{\delta}$ & $l l_{\text {Null }}$ & $d f_{\text {Null }}$ & $l l_{\text {Model }}$ & $d f_{\text {Model }}$ & $L R T$ & $p$ & $\operatorname{cor}^{2}(\hat{y}, y)$ \\
\hline 0.30 & 0.26 & -0.95 & -1813.6 & 4 & -178.6 & 13 & 3270.0 & $<0.001$ & 0.37 \\
\hline
\end{tabular}

\begin{tabular}{lrcrc}
\hline Covariate & Est. & $s e$ & \multicolumn{1}{c}{$t$} & $p$ \\
\hline Intercept & 4.67026 & 0.09416 & 49.6 & $<0.001$ \\
balPrY & -0.00640 & 0.00077 & -8.3 & $<0.001$ \\
BZ & 0.00384 & 0.00015 & 25.0 & $<0.001$ \\
prec & 0.00278 & 0.00024 & 11.7 & $<0.001$ \\
temp & -0.13918 & 0.00511 & -27.2 & $<0.001$ \\
year & -0.27649 & 0.00683 & -40.5 & $<0.001$ \\
cloneHYB & 0.08013 & 0.01032 & 7.8 & $<0.001$ \\
cloneAF2 & 0.03336 & 0.00953 & 3.5 & $<0.001$ \\
cloneING & -0.10870 & 0.00982 & -11.1 & $<0.001$ \\
cloneTOR & -0.05188 & 0.00985 & -5.3 & $<0.001$ \\
\hline
\end{tabular}

$\sqrt{\text { incrHRot } 2}=4.67026-0.00640 *$ balPr $Y+0.00384 * B Z+0.00278 *$ prec $-0.13918 *$ temp $-0.27649 *$ year $+\left(\begin{array}{c}0 \\ 0.08013 \\ 0.03336 \\ -0.10870 \\ -0.05188\end{array}\right) *$ clone 


\section{Growth of Trees with Height $\leq 1.3 \mathrm{~m}$}

After coppicing, some trees with a height below $1.3 \mathrm{~m}$ were observed although this was rarely the case ( $n=44$ trees). Because the BAL based on DBH can not be calculated in these cases and the RCD was not measured in the second rotation an additional model was parameterized to estimate growth for these trees. It was refrained from integrating the competition index BAL because the basal area could also not be calculated for the respective trees.

A simple linear model was fit to the data and the response was log transformed to meet the assumptions of normal distribution and homoscedasticity. After variable selection, only the two climate variables of mean sum of precipitation prec in May and June and mean temperature temp in June and July were identified as sufficient for estimating growth in this subset (see model summary in Table 2.20 . The predictive power is intermediate with $\operatorname{cor}^{2}(\hat{y}, y)=0.46$. The small sample size has to be minded though.

Table 2.20: Summary of linear model fit for estimating the height increment of trees with a height $\leq 1.3 \mathrm{~m}$ during the second rotation. The response was log transformed. Independent variables are the the mean sum of precipitation of months May and June prec and the mean temperature temp in that same period.

\begin{tabular}{|c|c|c|c|c|c|c|c|}
\hline$\hat{\sigma}$ & $R S S_{\text {Null }}$ & $d f_{\text {Null }}$ & $R S S_{\text {Model }}$ & $d f_{\text {Model }}$ & $F$ & $p$ & $\operatorname{cor}^{2}(\hat{y}, y)$ \\
\hline \multirow[t]{4}{*}{0.86} & 70.1 & 44 & 31.3 & 42 & 26.1 & $<0.001$ & 0.43 \\
\hline & Cov & ariate & Est. & $s e$ & $t$ & $p$ & \\
\hline & prec & & 0.04792 & 0.00829 & 5.78 & $<0.001$ & \\
\hline & teml & & -0.36599 & 0.05838 & -6.27 & $<0.001$ & \\
\hline
\end{tabular}

\section{Data Completion During Second Rotation}

Similar to the estimation of basal area based on root collar diameter during the first rotation, the tree-wise basal area as the sum of all stem-wise basal areas based on their diameter at breast height (DBH) has to be estimated by height during the second rotation. The methodology here is basically the same as during 
the first rotation. Figure 2.18 contains an illustration of the relationship between the DBH basal area and tree height as the length of the tallest stem $l$.

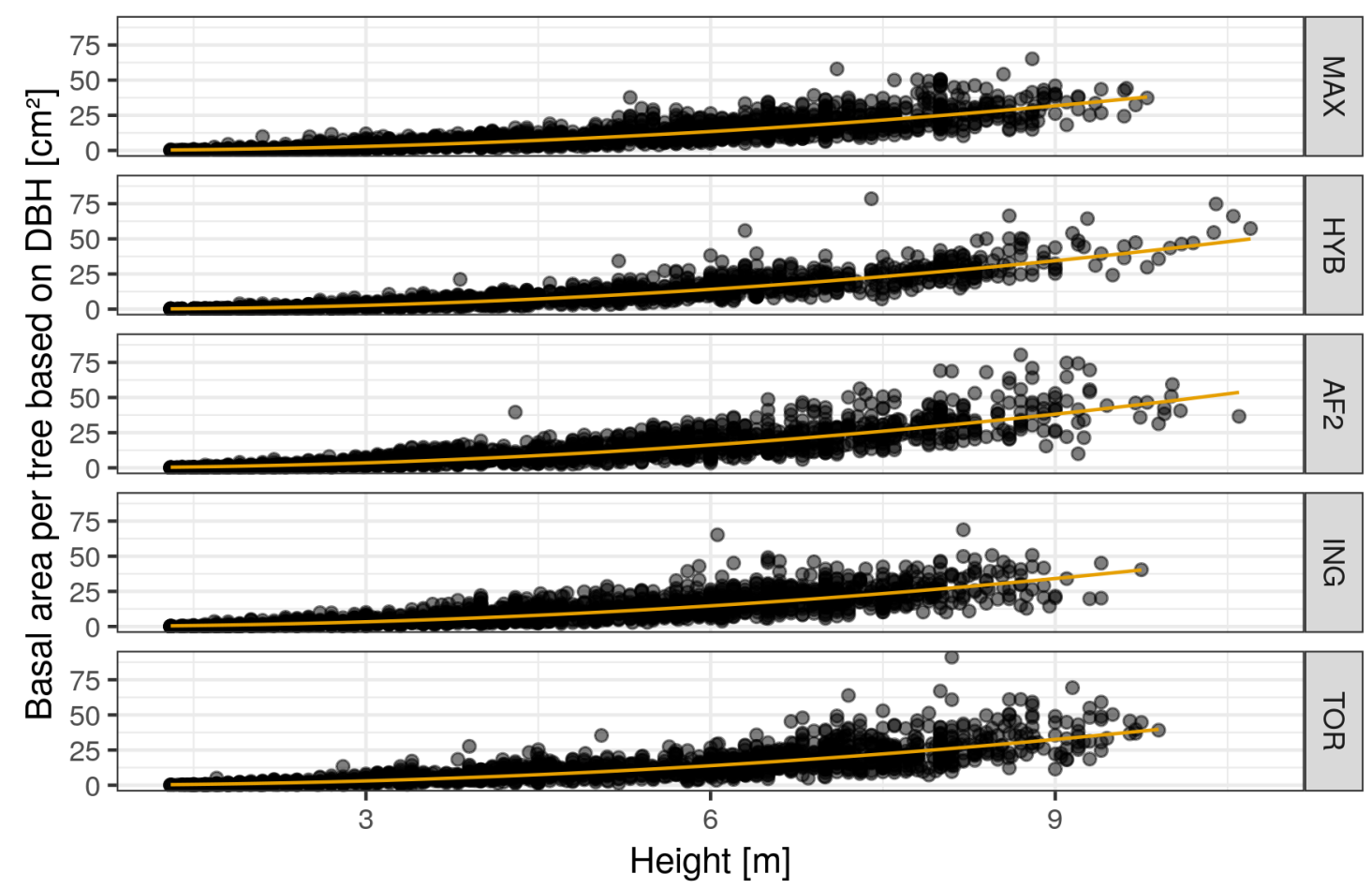

Figure 2.18: Relationship between tree-wise basal area calculated as the sum of single stem diameter at breast height (DBH) basal area and height as the length of the tallest stem per tree. The data is displayed separately by clone and only value pairs from the second rotation are presented here. The yellow lines resemble the back-transformed response from the fitted model.

The challenges in modeling this relationship were also similar to the first rotation. A square root transformation improved the residual structure regarding the nonlinear, positive correlation but still the increasing variance of the residuals and the correlation for repeated observations on the same tree had to be accounted for which was solved by using a linear model with GLS. The results of this model fit are contained in Table 2.21. The predictive power of the model with a $\operatorname{cor}^{2}(\hat{y}, y)$ $=0.79$ can be seen as good although it is lower than that for the RCD basal area estimation in the first rotation. Once more, the clonal effect and its interaction with height were identified as significant but practically do not seem to be too distinguished. 
Table 2.21: Summary of linear model fit using GLS for estimating the tree-wise basal area $d b h B a s$ based on the diameter at breast height (DBH) of all stems. Independent variables are the tree height as the length of the tallest stem $l$ and clonal affiliation clone. The response has been square root transformed. The colon sign stands for the interaction of two variables. The parameterized model function is given in Equation 2.22 .

\begin{tabular}{ccccrcrcrr}
\hline$\hat{\sigma}$ & $\hat{\Phi}$ & $\hat{\delta}$ & $l l_{\text {Null }}$ & $d f_{\text {Null }}$ & $l l_{\text {Model }}$ & $d f_{\text {Model }}$ & \multicolumn{1}{c}{$L R T$} & $p$ & $\operatorname{cor}^{2}(\hat{y}, y)$ \\
\hline 0.41 & 1.72 & 0.47 & -13627.3 & 4 & -6966.0 & 13 & 13322.6 & $<0.001$ & 0.79 \\
\hline
\end{tabular}

\begin{tabular}{lrcrr}
\hline \multicolumn{1}{c}{ Covariate } & \multicolumn{1}{c}{ Est. } & $s e$ & \multicolumn{1}{c}{$t$} & \multicolumn{1}{c}{$p$} \\
\hline Intercept & -0.28519 & 0.03266 & -8.7 & $<0.001$ \\
$l$ & 0.65825 & 0.00759 & 86.7 & $<0.001$ \\
cloneHYB & -0.19382 & 0.04739 & -4.1 & $<0.001$ \\
clone AF2 & 0.01461 & 0.04724 & 0.3 & 0.757 \\
cloneING & 0.10458 & 0.05013 & 2.1 & 0.037 \\
cloneTOR & 0.05154 & 0.05028 & 1.0 & 0.305 \\
$l:$ cloneHYB & 0.04695 & 0.01155 & 4.1 & $<0.001$ \\
$l:$ cloneAF2 & 0.05835 & 0.01130 & 5.2 & $<0.001$ \\
$l:$ cloneING & 0.01089 & 0.01161 & 0.9 & 0.348 \\
$l:$ cloneTOR & 0.00188 & 0.01124 & 0.2 & 0.867 \\
\hline
\end{tabular}

$$
\begin{aligned}
\sqrt{\text { dbhBas }}= & -0.28519+\left(0.65825+\left(\begin{array}{c}
0 \\
0.04695 \\
0.05835 \\
0.01089 \\
0.00188
\end{array}\right) * \text { clone }\right) * l \\
& +\left(\begin{array}{c}
0 \\
-0.19382 \\
0.01641 \\
0.10458 \\
0.05154
\end{array}\right) * \text { clone }
\end{aligned}
$$


Analogous to the first rotation, the mean annual increment during the second rotation is estimated based on the mean height after the sixth vegetation period. The relationship between both variables is, again, positive and nonlinear which can be confirmed in Figure 2.19.

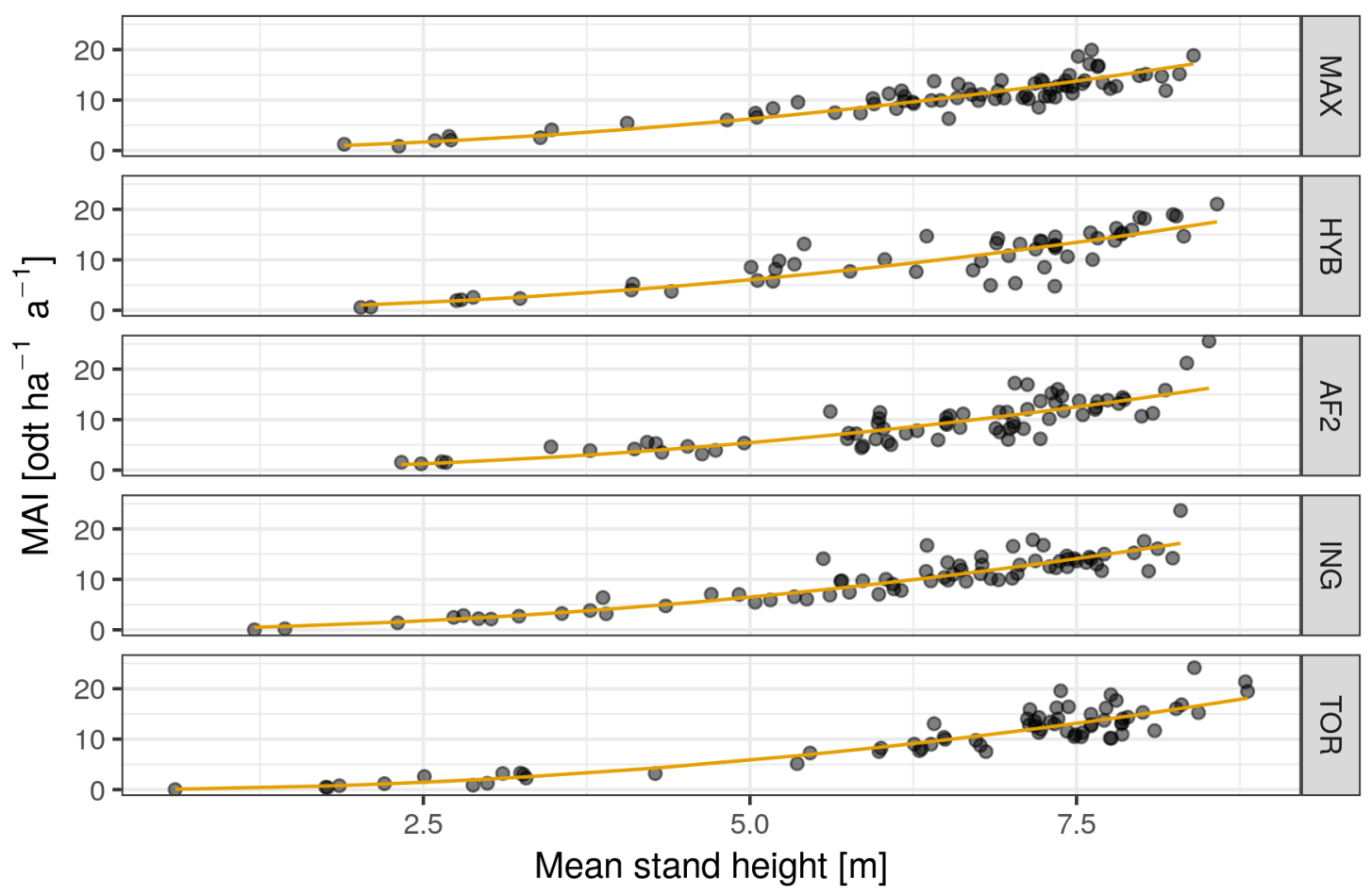

Figure 2.19: Relationship between mean stand height and mean annual increment at the end of the sixth vegetation period which is the end of the second rotation cycle. The data is displayed separately by clone. The yellow lines resemble the back-transformed response from the fitted model.

The fitted model reproduces the relationship quite well as indicated by the predictive power by $\operatorname{cor}^{2}(\hat{y}, y)=0.85$ which is given here instead of the $R_{\text {adj }}^{2}$ because the coefficient for the intercept did not test to be significantly different from 0 and hence was dropped from the model. The response was square root transformed as signified by the Box-Cox procedure which accounts for nonlinearity and increasing variance well. As it was the case for the first rotation, the differences between clones have practically little impact in estimating the MAI but were still left in the model after variable selection (see model summary in Table 2.22. 
Table 2.22: Summary of linear model fit for estimating the mean annual increment MAI during the second rotation based on dry mass after the sixth vegetation period. The response was square root transformed. Independent variables are the mean stand height $l$ Mean after the sixth vegetation period and the clonal affiliation clone.

\begin{tabular}{crrrrrr}
\hline$\hat{\sigma}$ & $R S S_{\text {Null }}$ & $d f_{\text {Null }}$ & $R S S_{\text {Model }}$ & $d f_{\text {Model }}$ & \multicolumn{1}{c}{$F$} & \multicolumn{1}{c}{$p$} \\
\hline 0.35 & 3446.4 & 334 & 39.2 & 328 & 4754.4 & $<0.001$ \\
\hline \multicolumn{1}{c}{ Covariate } & \multicolumn{1}{c}{ Est. } & \multicolumn{1}{c}{ se } & \multicolumn{1}{c}{$t$} & \multicolumn{1}{c}{$p$} \\
\cline { 2 - 7 } & lMean & 0.48347 & 0.01119 & 43.20 & $<0.001$ \\
& cloneMAX & 0.08442 & 0.08303 & 1.02 & 0.31 \\
& cloneHYB & 0.04242 & 0.08548 & 0.50 & 0.62 \\
& cloneAF2 & -0.08913 & 0.08172 & -1.09 & 0.276 \\
& cloneING & 0.13048 & 0.07918 & 1.65 & 0.1 \\
& cloneTOR & -0.00076 & 0.08314 & -0.01 & 0.993 \\
\hline
\end{tabular}

\subsubsection{Overall Simulator Performance}

After parameterizing the models, their combined, overall performance and predictive power needs to be evaluated by setting up the growth simulator procedure. Before implementing the simulator in Java, the simulation procedure itself was set up in $\mathrm{R}$ by creating a data frame that contained the needed combinations of clones and environmental conditions as in the raw data. The models were then applied sequentially to generate single trees and estimate their survival and growth over the course of two rotation cycles. The results after each rotation were compared with the raw data visually and by computing numerical summaries. The two stand parameters that were compared for assessing predictive power are the mean stand height, as length or its increment is the response of all growth models, and mean annual increment since this is the variable that is of most practical interest.

For mean stand height the differences between measured and estimated values are shown by estimated values in Figure 2.20 at the end of both rotations. The simulation procedure seems satisfactory for the first rotation. The positive differences above $1 \mathrm{~m}$, implying that the simulation procedure underestimated the actual mean stand height, are all for sites pl17 Unterrieden and pl25 Campus 
Klein Altendorf. The overestimated mean stand heights with a negative difference below $-1 \mathrm{~m}$ are mainly for sites pl04 Forchheim and pl19 Werlte. For higher predicted values (around $6 \mathrm{~m}$ ) there is a slight tendency for overestimation which completely relates to all clones in pl01 Emmendingen.

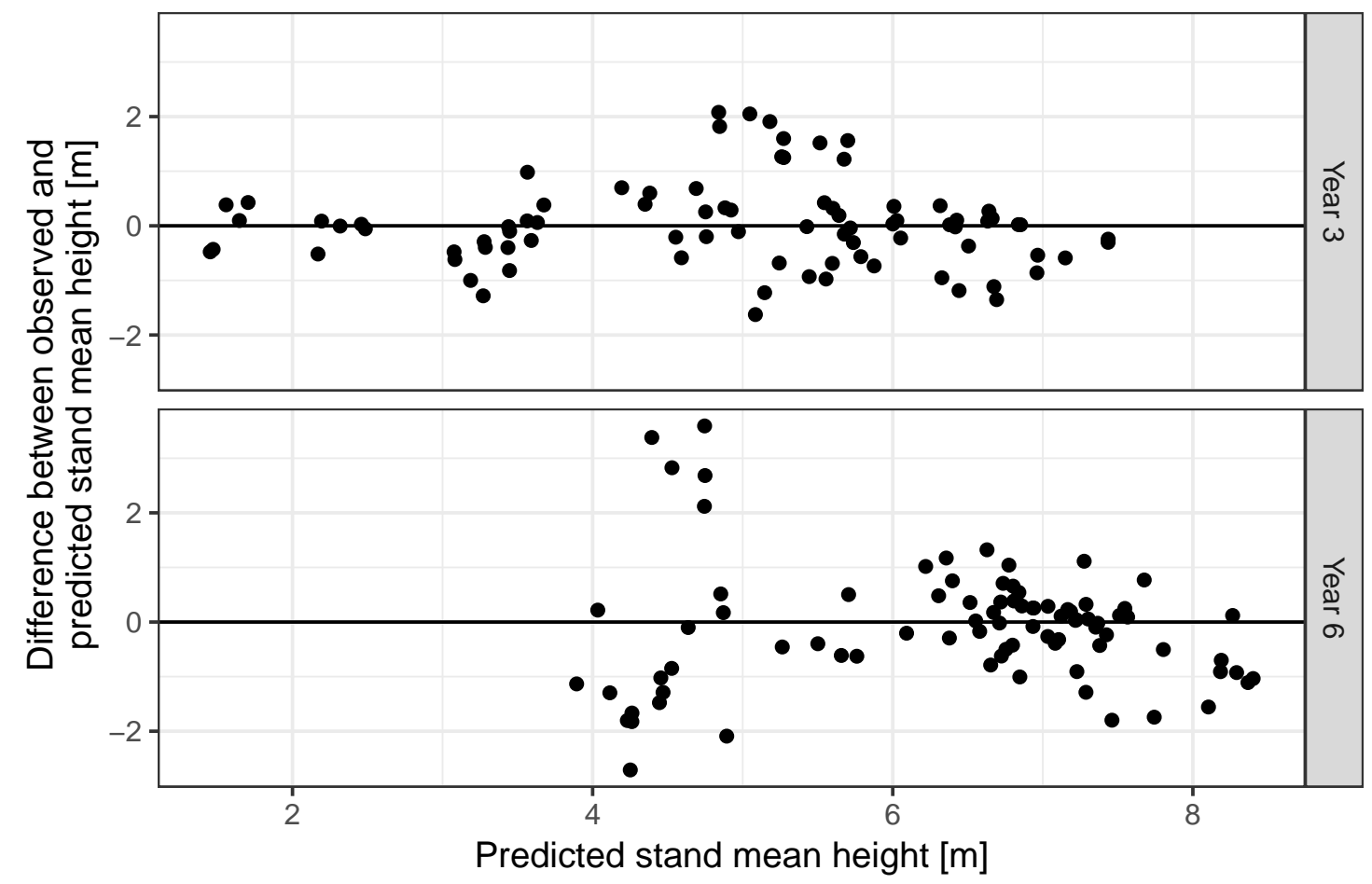

Figure 2.20: Differences between observed values and fitted values (comparable to residuals) by predicted values for mean stand height separated by year. Shown are only the years at the end of each rotation.

Within the second rotation the predictive power of the combined models deteriorates. Underestimated mean stand heights with a difference above $2 \mathrm{~m}$ occurred for sites pl15 Potsdam Bornim and pl25 Campus Klein Altendorf. The greatest overestimations (below - 2 m difference) occurred for stands pl04 Forchheim and pl30 Iden.

In Table 2.23 the numerical summaries for the deviation of observed and estimated values are shown. The trends described for the graphical display are reproduced here and can in part be reduced down to the overall correlation of observed and estimated values. The coefficient values are 0.79 at the end of the first rotation, which is comparably good, and 0.53 at the end of the second rotation which is intermediate. 
Table 2.23: Summary of the distribution of differences between observed and estimated values for mean stand height and MAI separated by year. Given is an extended five number summary with minimum/maximum value (Min, Max), the lower and upper quartile $\left(Q_{0.25}, Q_{0.75}\right)$, the median $(M d n)$ and the mean $(M)$. The last column contains the squared Pearson correlation $\operatorname{cor}^{2}(\hat{y}, y)$ between estimated $\hat{y}$ and observed $y$ values. Summary values have been rounded to 3 digits (except for correlation with 2 digits).

\begin{tabular}{rlccccccrr}
\hline Year & \multicolumn{1}{c}{ Variable } & $\mathrm{n}$ & Min & $Q_{0.25}$ & $M d n$ & $M$ & $Q_{0.75}$ & $\operatorname{Max}$ & $\operatorname{cor}^{2}(\hat{y}, y)$ \\
\hline 3 & Mean stand height [m] & 84 & 0.005 & 0.148 & 0.395 & 0.577 & 0.878 & 2.079 & 0.79 \\
3 & MAl [odt ha $a^{-1} a^{-1}$ ] & 84 & 0.003 & 0.362 & 0.890 & 1.292 & 1.823 & 4.785 & 0.70 \\
6 & Mean stand height [m] & 84 & 0.018 & 0.245 & 0.509 & 0.801 & 1.110 & 3.592 & 0.53 \\
6 & MAl [odt ha $a^{-1} a^{-1}$ ] & 84 & 0.050 & 1.065 & 1.997 & 2.548 & 2.939 & 11.841 & 0.42 \\
\hline
\end{tabular}

The numerical summaries for estimating yield as assessed by MAI are also given in Table 2.23. The MAI values are being estimated through the mean stand height, so the already described trends continue here and the bias from the model for MAl estimation (see models in Tables 2.15 and 2.22) adds to the existing inaccuracies. The correlations between observed and predicted values are therefore also lower for MAI than for mean stand height (see Table 2.23). The overall assessment that the simulation procedure outputs satisfactory results in the first rotation $\left(\operatorname{cor}^{2}(\hat{y}, y)=0.7\right)$ and intermediate results in the second rotation $\left(\operatorname{cor}^{2}(\hat{y}, y)\right.$ $=0.42$ ) remains. The graph in Figure 2.21 illustrates the distribution of differences by fitted values for the MAI. The same locations as with the height estimation are problematic here, especially for pl15 Potsdam Bornim and pl25 Campus Klein Altendorf which deviate from the observed values in the second rotation over 5 odt $h a^{-1} a^{-1}$. 


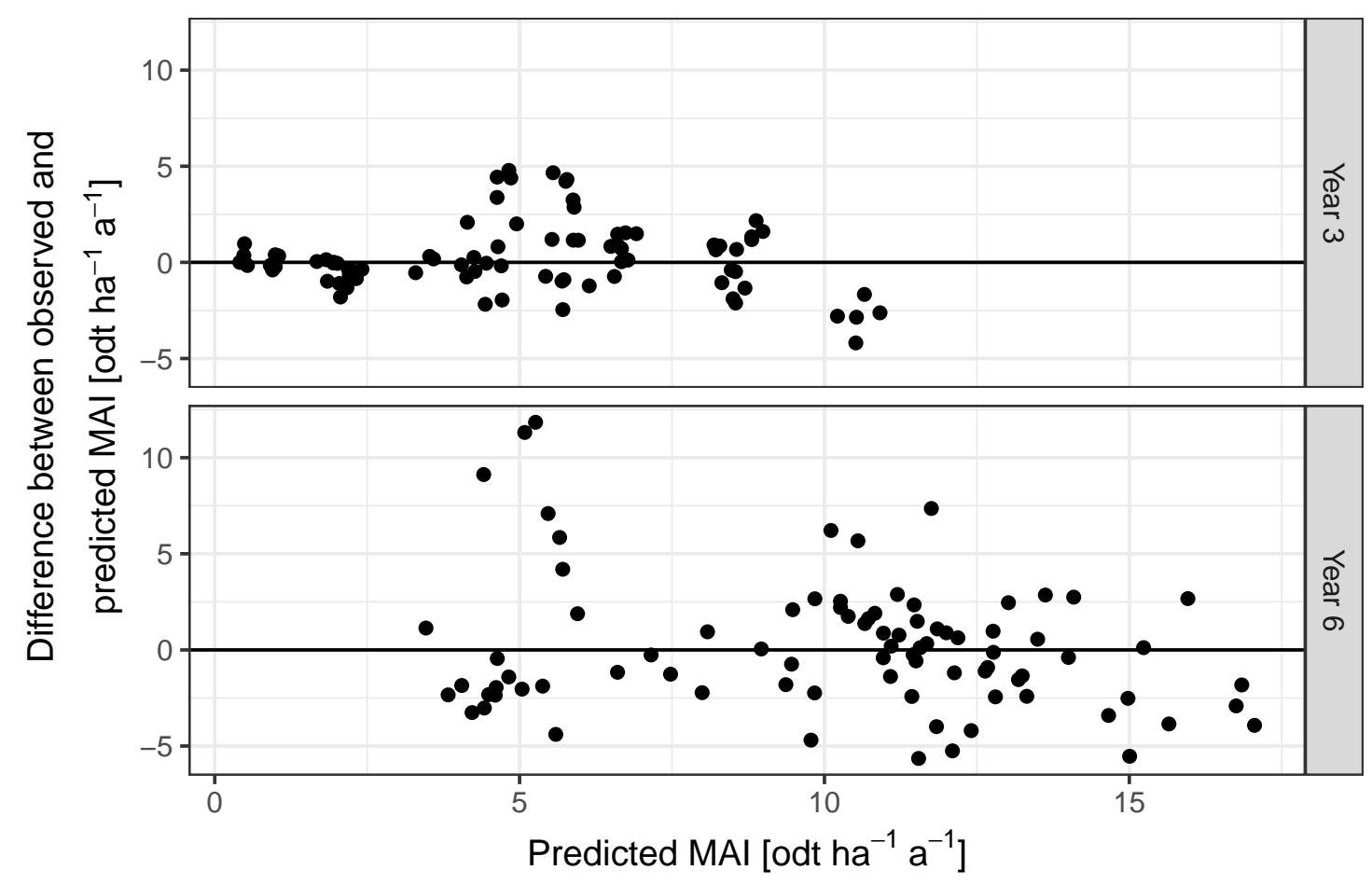

Figure 2.21: Differences between observed values and fitted values (comparable to resiudals) by predicted values for MAI separated by year. Shown are only the years at the end of each rotation.

The simulation procedure has in terms of accuracy still need for improvement, especially in the second rotation. With the described methodology and the present data the results could not further be improved significantly. The results will be further discussed in Section 5, 


\subsubsection{Simulator Implementation}

As described in Section 2.1.5, the yield simulator was implemented based on the TreeGrOSS library and the forest growth simulator BWINPro. The whole set of functionalities that this package offers is not used to full capacity in simulating $S R C$ growth. Aspects like different thinning regimes or anything relating to growth of mixed species stands is obsolete in the case of short rotation coppice forestry. Other factors were not considered due to the available data. The main distinctive feature is the coppicing and resprouting itself whose integration into the whole simulation procedure will be described in this Section. For a comprehensive description of TreeGrOSS and BWINPro the reader is referred to Hansen and Nagel (2014).

Figure 2.22 contains an overview of the 4 classes that were implemented to simulate the growth of single trees within an exemplary stand. Besides these classes, a main class was implemented that contains the graphical user interface (GUI) and calls the 4 other classes in the desired order to simulate the SRC stand. The procedure of this is depicted in Figure 2.23. In the following the basic principles of the simulator will be described. 

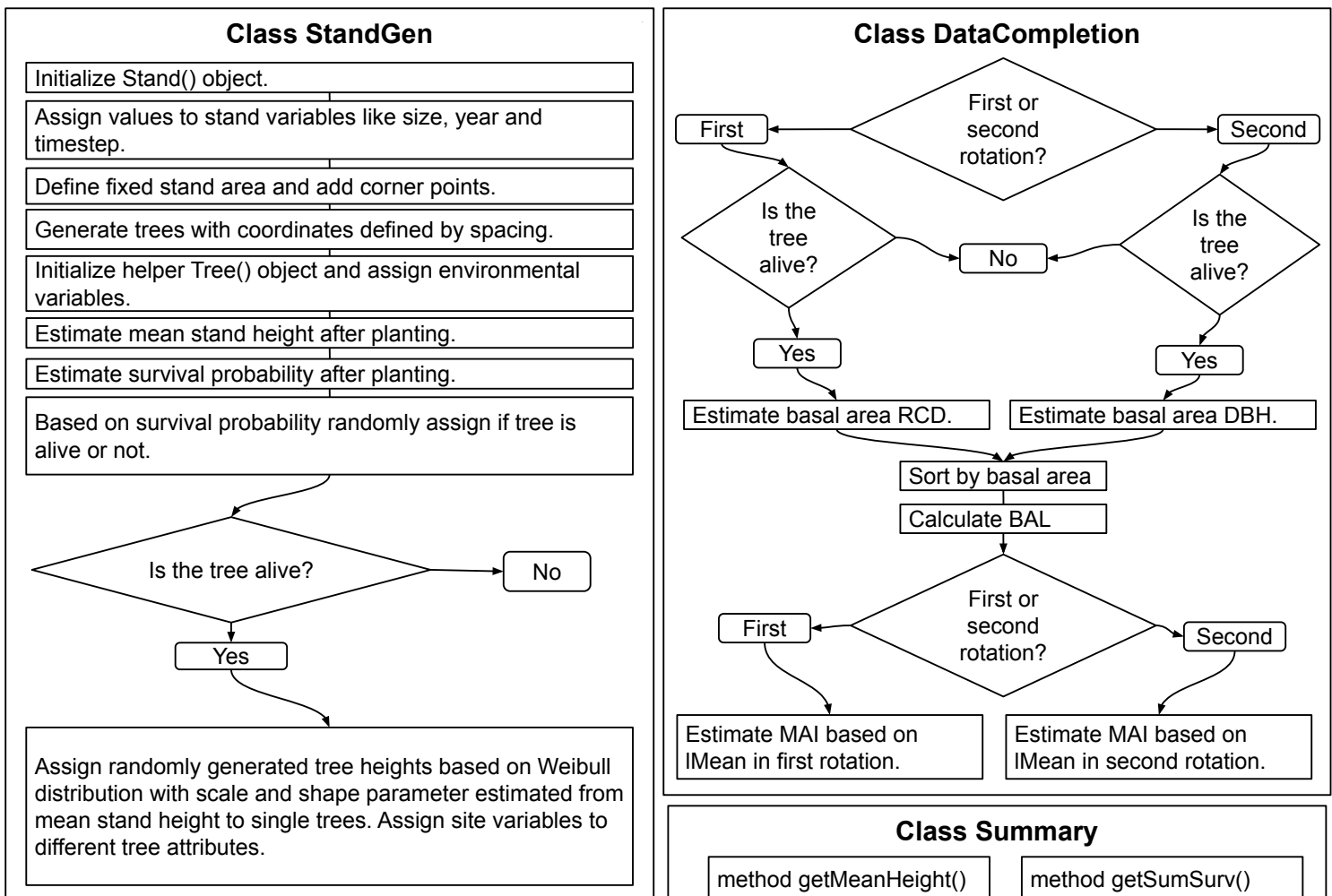

different tree attributes

\section{Class Summary}

method getMeanHeight() method getSumSurv()

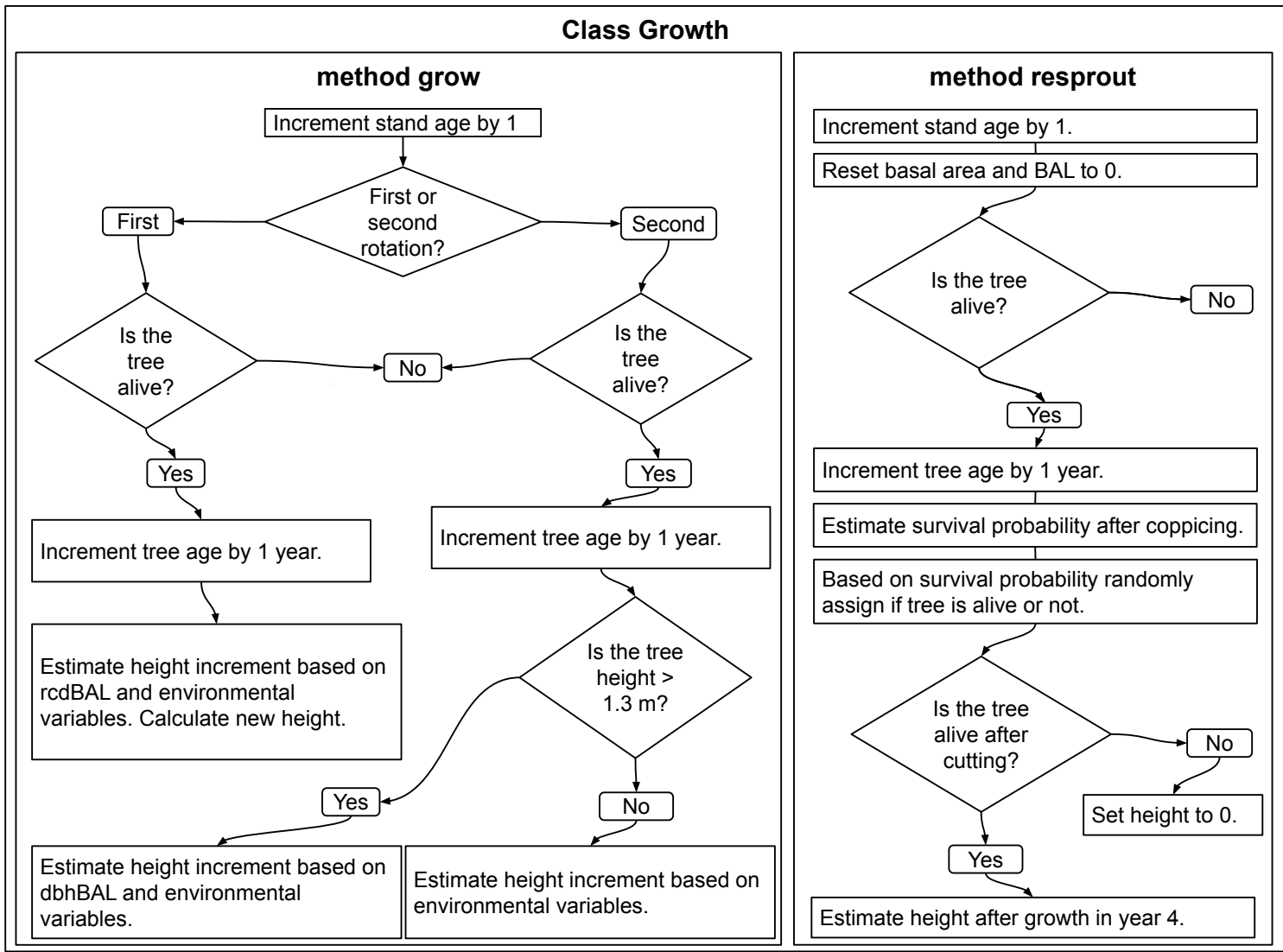

Figure 2.22: Overview and flowchart of the classes and their internal procedures and methods used in the growth simulator. 


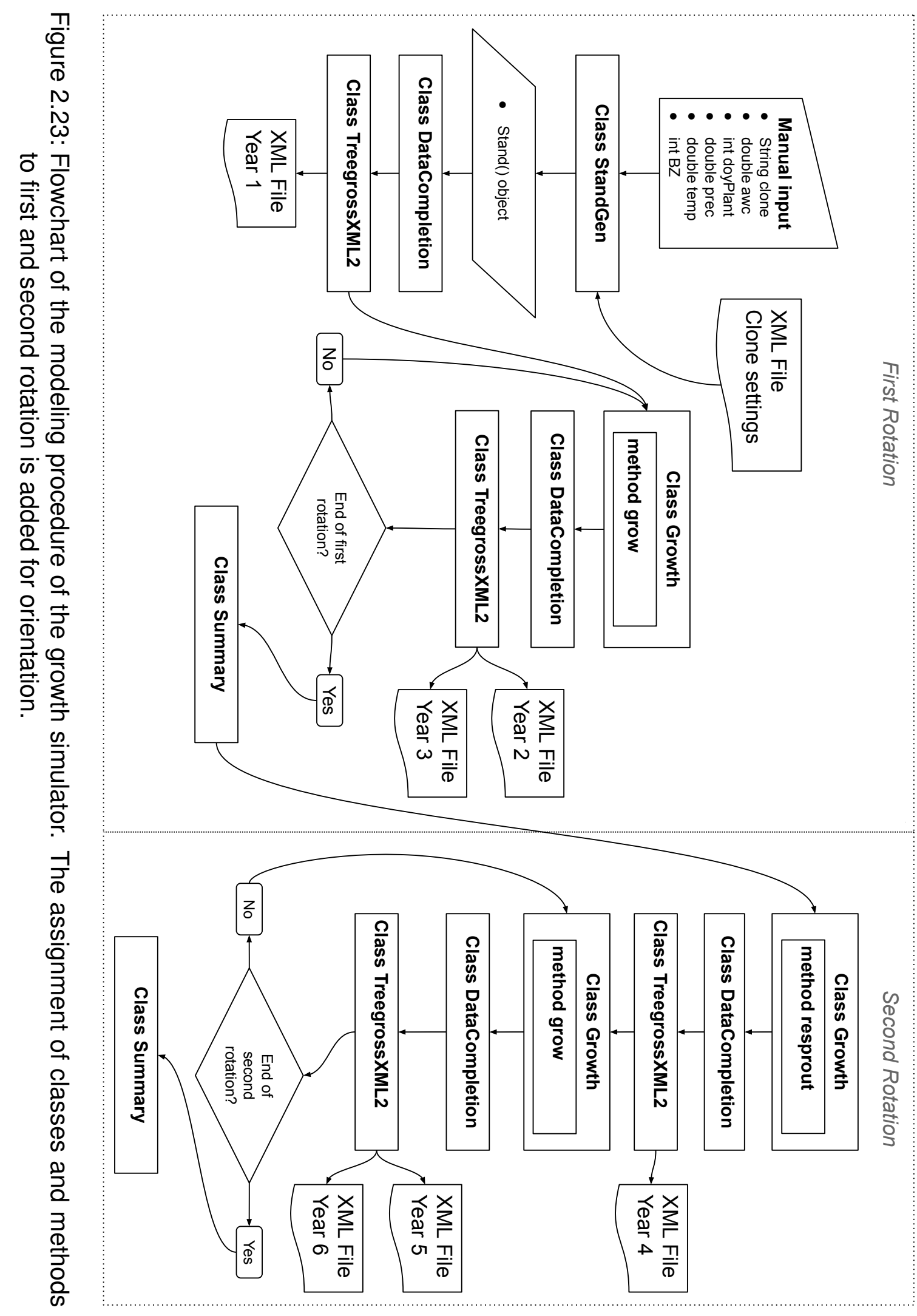


The most extensively used TreeGrOSS features are the Stand and inherently the Tree object. Furthermore the FunctionInterpreter plays a central role in parsing the model functions for estimation. The functions themselves have to be saved in XML species setting files. These are adapted from the BWINPro settings files which contain information on a tree species, like meta data, some general attributes and the functions that are needed to estimate the species specific growth. Again, not all BWINPro functions are needed here. The functions from the models in the previous section are superimposed on the default functions. For example, the XML element StemVolumeFunction that contained the function for estimating stem volume before, now includes the function for estimating the RCD basal area based on height. The XML setting files are required for starting the simulation. Furthermore, a manual input has to be made by specifying the environmental variables for the desired yield prediction. As soon as the user starts the simulation within the class StandGen a Stand object is initialized and general information is added to this object like the coordinates of the corner points of the plantation. Currently, the stand is initialized as a rectangle. Depending on the stand size, Tree objects are added to the stand in the spacing of $0.5 \mathrm{~m}$ within row and $1.8 \mathrm{~m}$ between row distances. This is repeated until the stand area has been fully covered with trees. It has proven feasible that not a full hectare should be simulated as this implies generating and handling 11,111 trees which causes problems with the built-in threshold of 8500 maximum trees per stand. A stand area of 0.25 hectare seemed appropriate. For using the interconnection however, an even smaller stand size should be chosen which will be discussed in Section 4.2. The tree object so far does not have any growth parameters specified. As a workaround, a single tree outside of the Stand object is generated which subsequently has the manually entered environmental variables added to it as properties. Using these properties, the function interpreter is then called to parse the function for mean stand height estimation (see model described in Table 2.10) and estimation of survival rate (Table 2.9), both after planting. The parsed function resorts to the environmental variables saved in the helper Tree object. Based on the survival probability, the single trees are randomly declared as alive or dead. Height values are then randomly assigned to the surviving trees via the Weibull distribution. The shape and scale parameter are estimated based on the mean stand height according to the models described in Table 2.11 and 2.12. For 
the following model applications the environmental variables set by the user are assigned to tree attributes that are, in opposition to BWINPro, not needed by the SRC simulator (like crown width). This concludes the execution of class StandGen. The returned Stand object is then passed on to the class Datacompletion. The class differentiates between first and second rotation. In both cases, a for loop iterates through the Stand object and, if a tree is alive, the basal area based on RCD or DBH depending on rotation (respectively to models summarized in Table 2.14 and 2.21) is estimated. Afterwards, the trees are sorted by basal area, followed up by calculating the BAL. This procedure is the same for the first and second rotation. The final step of the data completion differentiates between these two again by calculating the mean stand height and then estimating the mean annual increment based on it with the separately parameterized functions (see Tables 2.15 and 2.22). This concludes the data completion and also marks the end of the first year. Next, the Growth class with method grow is called. Via another for loop the age is incremented by 1 for all trees that are alive. Then the height increment is estimated based on the BAL and the environmental variables (model function based on coefficients from Table 2.13). In the second rotation two further cases are distinguished for height increment by whether a tree has a height $>1.3 \mathrm{~m}$ or not (see Tables 2.19 and 2.20). The grow method then returns the Stand object which is then passed on to class DataCompletion. The point in time is now the end of the second year, the procedure for the third year is the same. At the end of the third year, the Summary class is invoked, which calculates the survival percentage and mean stand height. Together with the MAI they are printed to the GUI window. At the intersection of the first and second rotation, as the biomass is harvested, the resprout method inside the Growth class is called. Height increment, basal area and BAL are set to 0 , except for height after year 3 since it is needed for calculating the new height (see Table 2.18) and the survival probability (see Table 2.17). For trees that are alive the survival probability is calculated and then, randomly, the trees are divided into still alive or dead. For the former case, the increment in the fourth year is estimated. Finally, the Stand object is returned by the resprout method. Subsequently, the routines of class DataCompletion are applied to the Stand object and its trees. This concludes the fourth year. As it was the case in the first rotation, the same cycle of grow and DataCompletion is being repeated till the end of the second rotation. At the end 
of the second rotation, the summary gets called again and the summary string is written to the GUI output panel.

This concludes the whole simulation procedure. After each year, an XML file is written to an output folder. These files are based on the BWINPro XML output format and contain general information on the stand like tree species, coordinates of corner points and stand age. Additionally, all the relevant information and data of all single trees are included. This is organised in elements and nested elements, element attributes are not used here. These files are later on used as the input for the interconnection with the structural model.

An example of the visual appearance of the GUI, using the Java Swing toolkit, is given in Figure 2.24 .

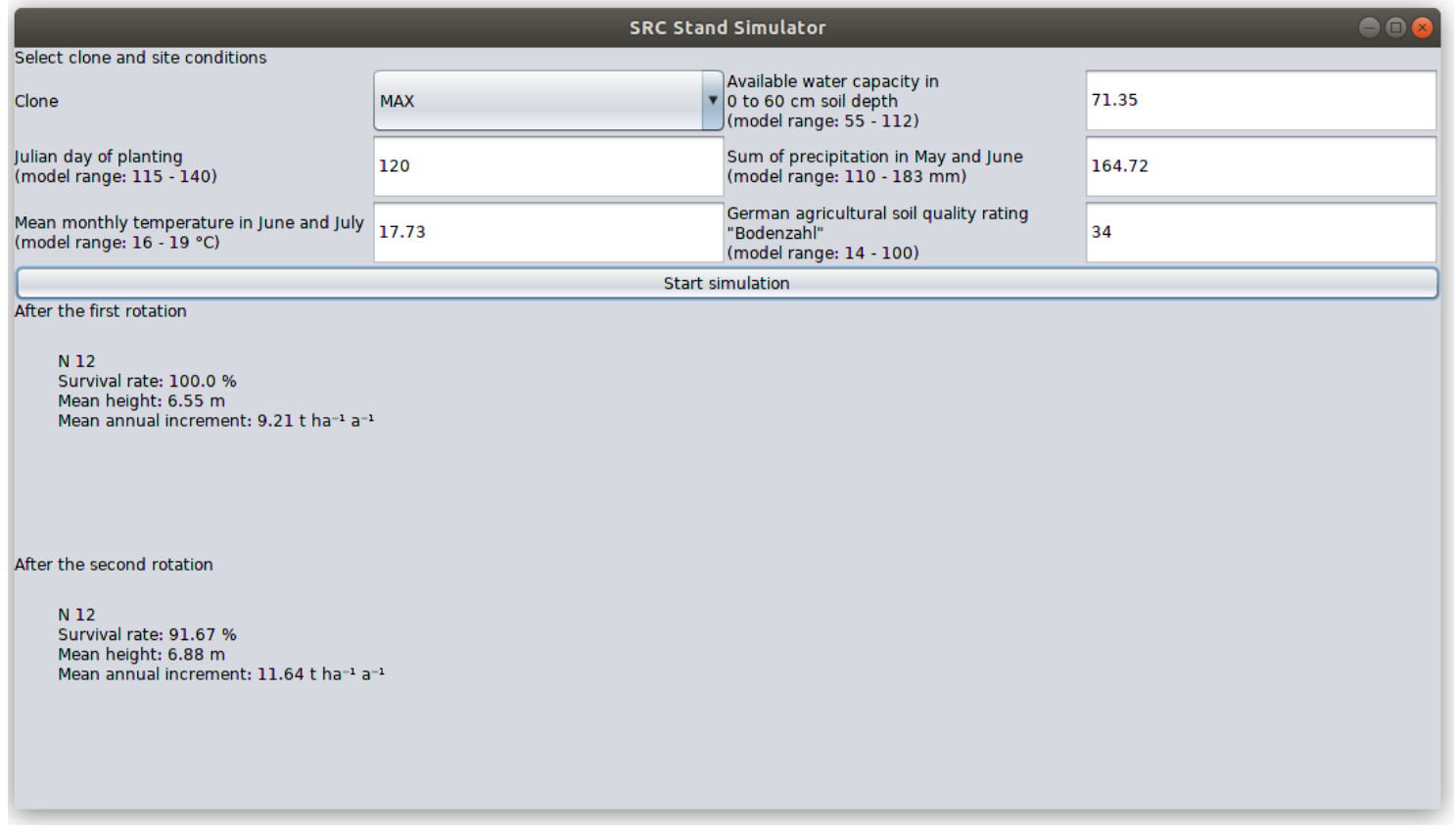

Figure 2.24: Exemplary display of the GUI of the yield simulator. Entered here is the environmental data for location pl31 Pommritz. The simulation has been executed and the results are displayed in the lower part of the program window. The number of trees is set to $N=12$ here as it is needed for the interconnection with the structural model.

Compared to the BWINPro implementation, the GUI here is very simple. Further information on license, version and a short manual with hints for the model limitations will be made available within the program. The information on how to obtain the source code of the simulator is given in the Appendix. 


\section{Structural Model}

\subsection{Material and Methods}

To encompass the aspects of tree structure regarding woody above-ground biomass and leaf organs, four separate measurement/data acquisition campaigns were conducted from 2014 to 2017. In the given order the campaigns focused on tree structure (as structure of woody above-ground biomass) in general, tree structure with a focus on branch curvature, leaf position and structure as well as leaf morphology with an emphasis on leaf shape. In this section some general considerations on measuring structure will be made, followed by a brief description of the trial sites where the measuring took place. Next, the methodology of all campaigns will be described in detail and finally some remarks on the software used for statistical and structural modeling will be made.

\subsubsection{Methods for Measuring Tree Structure}

For forestry research involving three-dimensional data Surový (2017) establishes a differentiation of surface and structural data. The former relates to the sole acquisition of surface point data to quantify an object's location or extension in 3D space. This does not include gathering information on the object or its entity itself (Surový, 2017). Surový (2017) lists point cloud data from stereo photogrammetry or laser scanning or sparse point fields gained through contact measurement as examples. The advantages for this type of data and its inherent methodological approach depend on the study goals which in forest growth research commonly refer to estimation of stand volume. In comparison to classical manual measuring approaches, gathering and utilizing surface data is less labour intensive in the field and hence faster while being more precise, more objective and non-destructive (Seidel et al., 2012; Kędra et al., 2019). Surový (2017) also men- 
tions the lesser requirements in biological knowledge when measuring as well as simpler and faster visualization possibilities. Especially terrestrial laser scanning (TLS) is experiencing rapid growth in utilization and development for forest inventory and research (Seidel et al., 2011; Liang et al., 2016). The applicability of TLS for estimating basal area in short rotation coppice plantations has already been proven by Seidel and Ammer (2014). However, the main problem in utilizing 3D point cloud surface data from TLS for structural modeling lies within the automated reconstruction of branch structure which becomes increasingly difficult and less accurate with decreasing branch size and increasing distance from scanner to branch due to occlusion within the tree crown (Pyörälä et al., 2018; Eysn et al., 2013; Boudon et al., 2014). Structural data, as the name suggests, contain information on the structure of organisms like plants or trees (Surový, 2017) which, as already established, implies not only the location and extension in 3D space but also the relation of components. Surový (2017) lists the advantages of this data type as the possibility to quickly gather insight on the architecture of an object and the easier processing of this kind of data. In comparison to surface data Surový (2017) evaluates structural data as more suitable for implementing models.

For measuring the 3D structure of trees several methods and their inherent data formats are available. Surový (2017) refers to Danjon and Reubens (2008) who differentiate 3 categories of methods for measuring root architecture which can be applied to measuring above-ground structure of trees as well. The categories by Danjon and Reubens (2008) are:

- manual

- semi-automatic

- automatic

Danjon and Reubens (2008) further differentiate the first into measuring the 3D coordinates of tree components or measuring the length and orientation by angles (azimuth, elevation) of objects. While the former entirely relies on some external means for reference (e.g. a frame that needs to be constructed around the to be measured object) the latter does not entirely rely on such installations because the topology is inherently included which can be used to gradually reconstruct the 
architecture (Danjon and Reubens, 2008). This also implies advantages in measuring speed (Danjon and Reubens, 2008). Two approaches for coding data from such measurements are the multi-scale tree graphs (short: MTG, for reference see Godin et al. (1997); Pradal and Cokelaer (2009)) and the descriptive tree data format (short: dtd, for reference see Kurth and Anzola Jürgenson (1997); Kurth (1994a)). The decision was made in favor of the dtd format because of its existing integration into GroIMP and the already made good experience in using this format. The dtd format demonstrates versatility in allowing the specification of different plant organs like leaves and special structural features like short shoots. It can also be applied to below-ground biomass as done by Oppelt et al. (2001) for representing root structure which is not considered or further discussed here.

Considering the semi-automatic and automatic approach for measuring architecture, Danjon and Reubens (2008) further differentiate these categories. In the following only the options that were deemed suitable for measuring above-ground biomass for this work are listed:

- semi-automatic

- Utilizing a 3D contact digitizer

- Utilizing a digital compass and inclinometer

- automatic

- Enhancing surface data with topology information

Each item in this list has further differing implementations itself to achieve the same or similar goals.

The last option mentioned for automatic data acquisition is not fully automatic but requires manually adding structural information as long as branch detection algorithms are not fully capable of doing so (Danjon and Reubens, 2008). Bayer et al. (2013) have conducted a study using TLS for acquiring point cloud data which is then subjected to a process called skeletonization which is semimanually defining branch segments. This allows for the analysis of some structural features. The two options listed above for the semi-automatic approach have already been successfully applied. As an example for the second option, although for root architecture, Oppelt et al. (2001) used a digital compass and digital caliper to record spatial orientation with segment length and diameter to 
separate files which were then merged into the dtd format. For the first option, using a 3D contact digitizer, more publications are available. Chambelland et al. (2008) combined the 3D contact digitizing with laser scanning to quantify leaf morphology of young beech trees. Surový et al. (2011) used 3D contact digitizing for reconstructing the growth of two stone pine trees. Yoshimoto et al. (2014) used contact digitizing for assessing stem shapes of three tree species in Japan. The methodology of Dinis (2014) falls into the same category although the purpose here was to examine the root system of cork oak. For measuring structure, the three latter studies all successfully used the same hardware which is the electromagnetic motion tracker device FASTRAK $\AA$ by Polhemus (Colchester, Vermont U.S.A.). An addon to use the device through the 3D modeling software Blender (Blender Foundation, 2012) was developed at the department of Ecoinformatics, Biometry and Forest Growth by Wasilczuk (2012). Both device and addon were readily available for this research.

All described methods were evaluated for their suitability and applicability in measuring tree architecture encompassing the structure of woody above-ground biomass and leaves. The aim was to start with an approach that is easy to employ within the field, allows for a fast representation of tree structure and yields data that is easy to use for further processing and analysis. The automatic category was soon ruled out in the decision process since the needed TLS hardware was not available and purchasing it appeared to cost-intensive. Due to these reasons and because the manual approach is easier and faster to set up, the dtd format was chosen for the initial part of the structural measurement. Furthermore, the simpler data processing and analysis properties and the already existing experiences spoke in favor of this approach. After completing the manual measurement it became obvious that some aspects like branch curvature needed to be accounted for that could be examined more easily with the semi-automatic approach. Because the proper hardware was available the already existing structural data was extended. This will be elaborated in more detail later on.

Due to its importance for carbon allocation and hence biomass production and also the high variety for poplar genotypes (Van Volkenburgh and Taylor, 1996), leaf morphology was regarded as essential for a structural model, especially if further extensions would incorporate a physiological component. The measurement was divided into a campaign for leaf position and structure and another 
campaign for leaf shape. The former were measured by a manual modified dtd approach. The semi-automatic procedure seemed too difficult to apply because of the delicate structure of leaves and the necessary manual physical contact with the FASTRAK device which can easily lead to distortion (Danjon and Reubens, 2008; Surový, 2017). Additionally to leaf structure and orientation, differences in leaf shape became evident early and demanded an integration into the structural model for proper morphological representation. To digitize leaf shape for contour modelling the approach by Henke et al. (2014) was chosen. Henke et al. (2014) developed a procedure for repeatedly measuring leaves within the field, digitising their shape, modeling leaf size and contour dynamically in respect to growth and implementing the results in XL and GrolMP. The methodology that was applied for measuring leaf properties will be described in more detail later on.

The level of detail or scale for all described approaches was chosen accordingly. For manually measuring the structure and branch architecture the growth unit (short: GU) was defined as the basic morphological component. Hallé et al. (1978) first coined the term "unit of extension" which is defined as "a morphologically discrete growth increment" (Hallé et al., 1978, p. 391). Sievänen et al. (2000) define a growth unit as "the part of the shoot resulting from uninterrupted extension growth" (Sievänen et al., 2000, p. 403).

Room et al. (1994) describe a "Unit of growth" as "A morphologically discrete growth increment, the result of one episode of rhythmic growth by a module, i.e. extension of the performed contents of a previously dormant apical bud followed by growth of neoformed leaves (if any) and formation of a new, dormant, apical bud" (Room et al., 1994, p. 110).

In retrospective analysis of rhythmic growth these growth units can be delimited by identifying the shoot base (bud scale) scars (Room et al., 1994; Roloff, 2001). These can be quite easily identified on shoots of young poplar trees (see Figure 3.4. panel (d)).

For the semi-automatic measurements the scale detail was increased by focusing on the internodes through measuring the nodes. In leafless condition this was done by digitizing the location of the buds. For leaf structure and position the internode and, at its distal end, leaf with the latter consisting of leaf petiole (or stalk) and leaf blade (or lamina) were considered. In terms of a morphological unit this is referred to as a metamer (Caraglio and Barthélémy, 1997, as cited 
in Sievänen et al., 2000). Leaf shape was trivially assessed on the leaf level by digitizing leaf petiole and leaf blade.

Regarding the temporal scale repeated measurements through non destructive/non invasive methods would have been best for gaining the data foundation which properly resembles the growth dynamics in poplar. This was however not possible due to the demand in time and effort. Some of the growth dynamics can be reconstructed for prior years (Danjon and Reubens, 2008) as it has been done for example by Surový et al. (2011). The decision was made to measure data at the end of the second rotation since this was the focus of the ProLoc II project. The points in time for all field campaigns are included in the respective descriptions which are made in the following sections.

\subsubsection{Trial Sites}

All measurements of structural data were conducted on 3 trial sites in northern Hesse. Trial pl17 Unterrieden was part of the ProLoc AP1 trial series while the locations fw11 Stiedenrode I and fw15 Stiedenrode II were part of two trial series in the FastWOOD breeding program. Information on the trial locations are given within section 2.1.2. In figure 3.1 a map is included with the localization of all 3 trial plantations.

All 3 poplar clones of interest are part of each trial. The differences between the sites relate mainly to environmental conditions. Further minor differences exist due to trial management, e.g. different year of establishment, different number of replications, different plot size and different spacing $(1.8 \mathrm{~m} \times 0.5 \mathrm{~m}$ versus 2.0 $\mathrm{m} \times 0.5 \mathrm{~m})$. These differences are unfortunate but could not be inhibited since the trials belong to series that were established with different aims. The structural measurements had to rely on multiple sites as a space for time-substitution due to organizational reasons. Nevertheless, the overall differences in tree structure between sites seem negligible here due to using clonal material. 


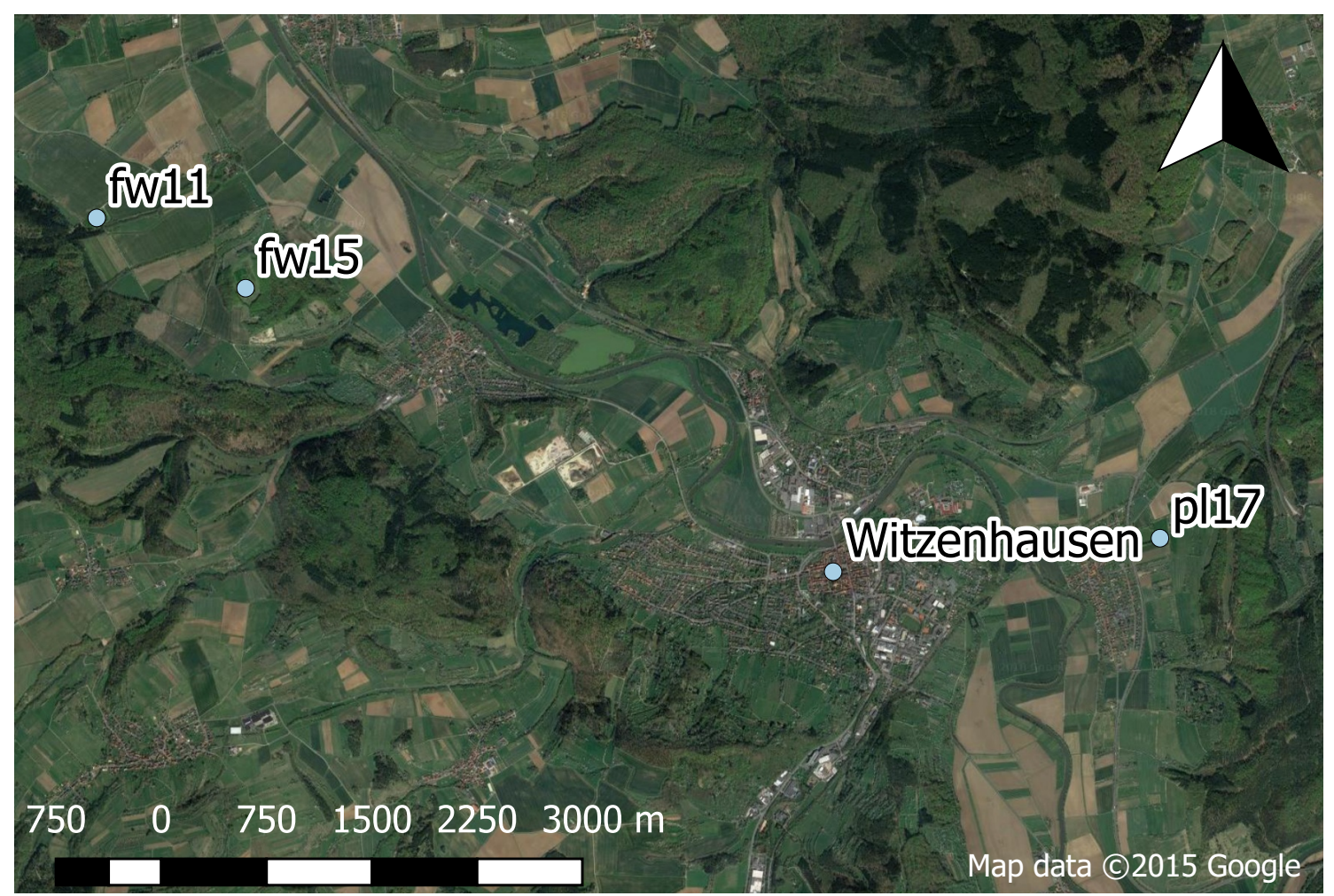

Figure 3.1: Location of all trial sites where measurements for the structural model were carried out. The city of Witzenhausen is marked for orientation. Satellite image by Google Satellite (2015)

\subsubsection{Tree Architecture}

\section{Structural Data from dtd Measurement}

During the late winter of 2013/2014 the structure of multiple trees was manually measured with the dtd approach at the ProLoc trial site pl17 Unterrieden. The measuring took place from January to March 2014 with the rotational second harvest in Unterrieden. An overall amount of $n=51$ trees were examined. 3 different levels of detail and different types of measurement were differentiated:

1. Detailed morphological measurement

2. Specific measurement with focus on stems from resprouting

3. Summary measurement with focus on main stem

Following the $\mathrm{dtd}$ methodology the first type of measurement included data acquisition according to the variables in Table 3.1. During the modeling process, 
the branch age was additionally re-coded to the year $t$ that the shoot formed in. This is basically the inverted age sequence: At the time of measurement (after the third vegetation period) shoots from year 1 are 3 years old, shoots from year 2 are 2 years old and shoots that have lengthened during the last vegetation period, which is the third one, are 1 year old. In contrast to the yield model, the years are referred to here as the within rotation years. Since the structural model focuses on the second rotation the years that are numbered 4 to 6 in the yield model are here referred to as 1 to 3 .

Table 3.1: All parameters that where measured for branching architecture during early 2014 on a single growth unit level.

\begin{tabular}{lll}
\hline Variable & \multicolumn{1}{c}{ Description } & Unit \\
\hline idGU & Identifier of GU incorporating plot-ID, tree-ID, stem-ID and GU-ID & \\
idMother & Identifier of mother GU, incorporating the same data as idGU & \\
I & GU Length & {$[\mathrm{mm}]$} \\
$\mathrm{d}$ & GU mid-diameter & {$[\mathrm{mm}]$} \\
$\mathrm{A}$ & Position of insertion / insertion height on mother & {$[\mathrm{mm}]$} \\
V & Whether the GU is a prolongation of another GU & \\
W & Branching angle & {$\left[{ }^{\circ}\right]$} \\
$\mathrm{E}$ & Number of internodes & {$[\mathrm{n}]$} \\
$\mathrm{K}$ & Number of living internodes & {$[\mathrm{n}]$} \\
order & Branch order (only recorded for order 0, rest computed afterwards) \\
age & Branch age (only recorded for order 0, rest computed afterwards) & {$[\mathrm{a}]$} \\
$\mathrm{t}$ & Year that the shoot lengthened in (re-coded from age & {$[\mathrm{a}]$} \\
$\mathrm{R}$ & Directional angle in classes (see Figure 3.2 & {$\left[{ }^{\circ}\right]$} \\
\hline
\end{tabular}




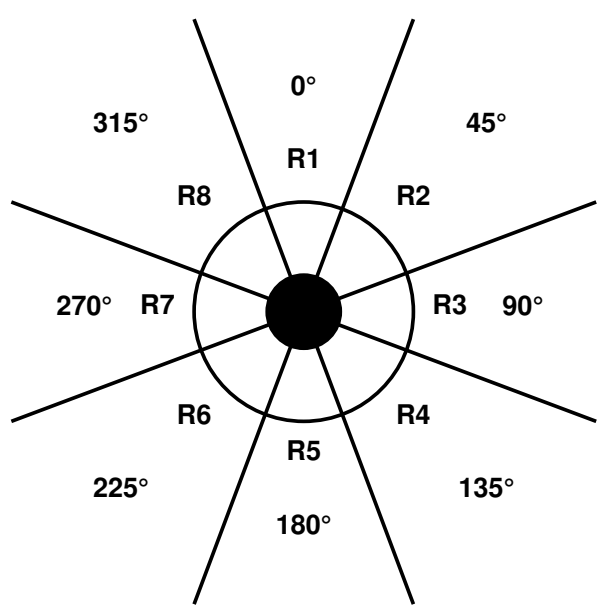

Figure 3.2: Scheme for dividing the directional angle into 8 sectors. When looking along a GU sector 1 resembles the upward facing direction. For vertically upward pointing GUs sector 1 is aligned with the northern compass direction (Graph and description adapted from Kurth, 2010).

On the basis of Figure 3.3, a short example should illustrate the basic functionality of the dtd format. The graphical output of GroIMP from an imported dtd file is shown and has been annotated afterwards. The depicted upper part of a tree originates from actual structural measurement of a tree from trial site pl17 Unterrieden (Main stem of tree 1.4.175). Each row of a dtd file contains information for a single growth unit. The data is whitespace-delimited. The focus is set on GU number 98 to exemplify how structural information is stored within dtd files:

\section{ID98 L1192 \#3 A1100 R6 W61 D5.95}

The GU has a length of $1192 \mathrm{~mm}$. Its mother is GU number 3, the insertion point on its mother GU is at a length of $1100 \mathrm{~mm}$ (GU 3 has a total length of $1304 \mathrm{~mm}$ ). The directional sector along the mother GU is 6 (not labeled in figure 3.3 ) and the branching angle is $61^{\circ}$. If a growth unit is a prolongation of its mother, implying that it inherits the same branching order, a " $\mathrm{V}$ " is noted and specifications on branching angle and directional sector are omitted. The latter applies for growth units 3 and 4 in 3.3 as they belong to the main stem. Length or diameter data does not need to be specified in $\mathrm{mm}$, other units are valid as well. However all data has to be stored with the same unit, different units for diameter and length e.g. cause contorted visualizations. 


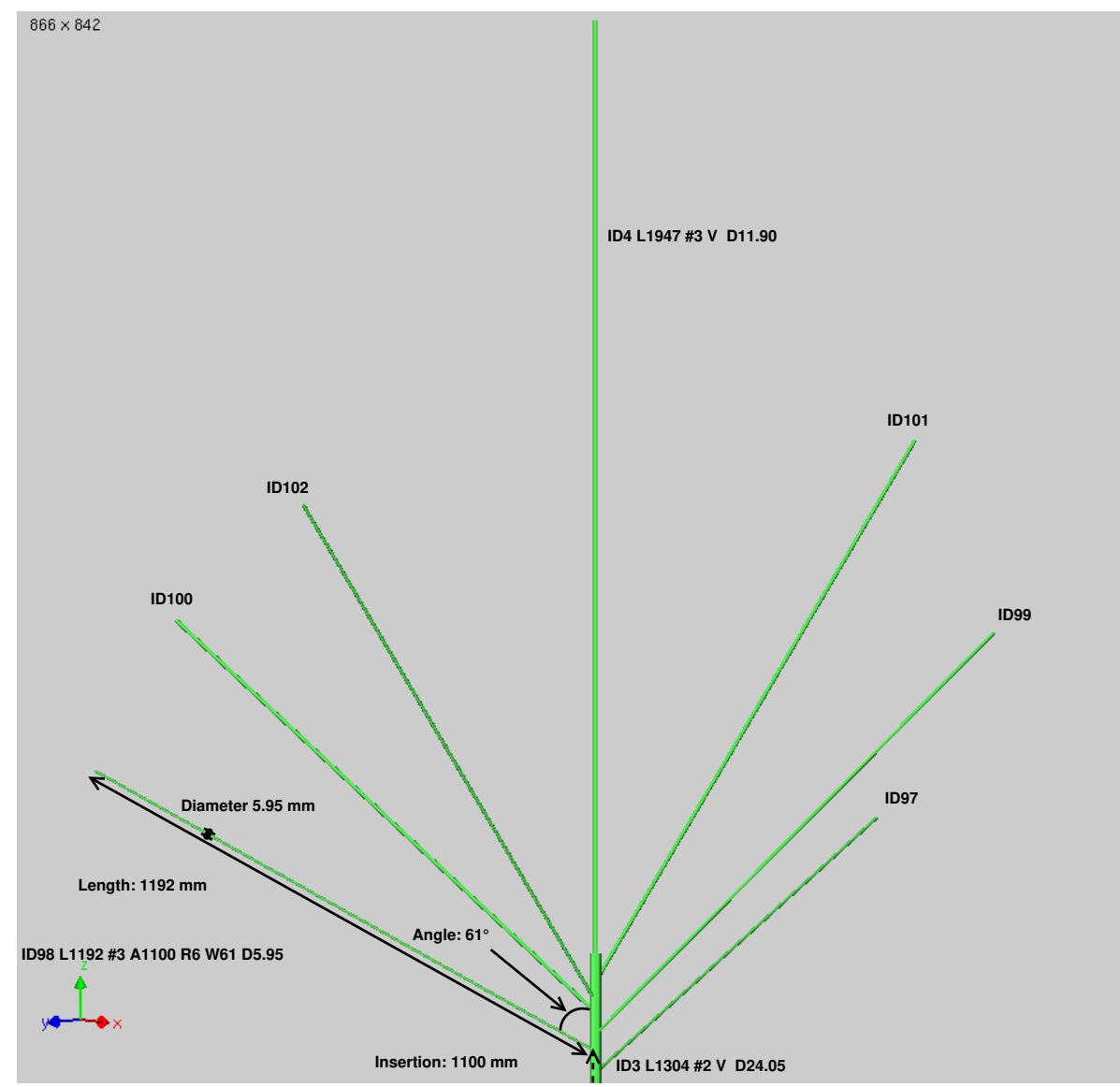

Figure 3.3: Example for dtd coding for tree 1.4 .175 stem 1 in pl17 Unterrieden. 8 growth units are displayed, other growth units were deleted for better visibility. 


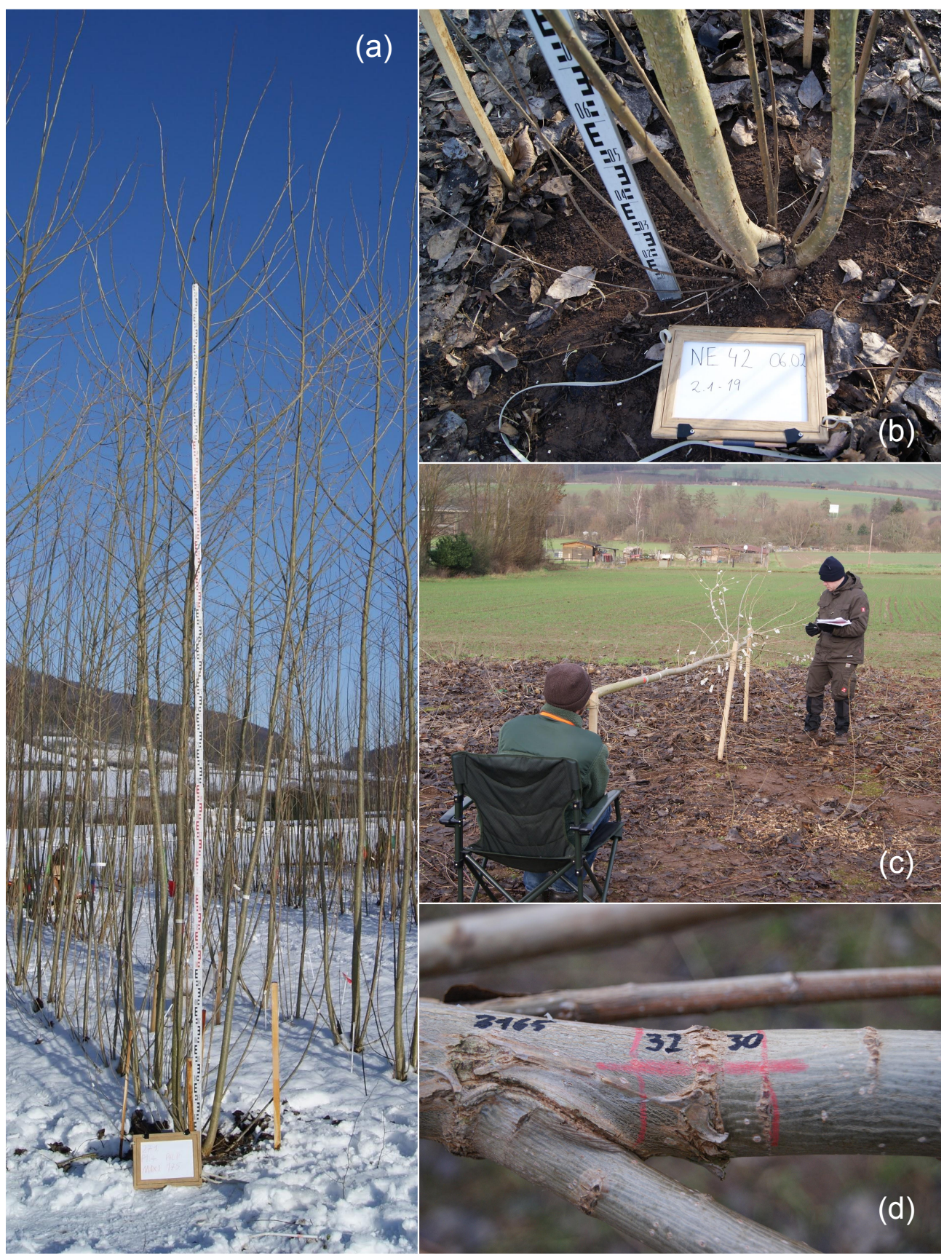

Figure 3.4: Exemplary display of some steps in manual measuring. Panel (a) shows a tree of the clone 'Max 1' photographed from the southern direction facing north. The trees in the plot margin have already been removed. Panel (b) contains a close up of the root stock of a 'Hybride 275' tree. The pole on the left marks the north direction. In panel (c) a cut off stem of one of the trees is fixed horizontally for measurement. Some of the labels that were applied to the GUs for identification are visible. Panel (d) shows further marks made directly on the stem for orientation. Shown is the scar left at the shoot base between a prolongation GU and its mother GU. 
A sample of $n=4$ trees per clone, i.e. $n=12$ trees collectively, were measured. Before commencing measurement, it was advisable to clear the surrounding ground area of the tree from litter and branches. Then the compass directions were marked with wooden poles. The stool of each tree was photographed up close with a small board containing date and tree identifier. With the same board placed appropriately, the tree was then photographed as a whole from the $4 \mathrm{com}$ pass directions (see panels (a) and (b) in Figure 3.4). Next, some information on the tree level was collected like overall tree height, breast height diameter of all stems and basal diameter. Since poplars, as already stated in contrast to willows, usually tend to develop one vigorous main stem and several minor stems after coppicing (Crow and Houston, 2004; Bärwolff et al., 2012; Janßen et al., 2017) an assessment was made which was the dominant main stem for each tree. The priorities in evaluating this were primarily focused on height and secondarily on diameter. This differentiation appears arbitrary but was actually needed for the modeling procedure. The implications will be discussed in Section 5. Following up, each stem's orientation was quantified by first consecutively numbering the stems and then assessing the directional angle $R$ and the orientation angle (angle to vertical) $W$ of all stems at root collar/stool level. Since their growth is predominantly upright, the growth direction of the main stems was assumed to follow a normal vector to the $x y$-plane which is the ground here. This implies no value for angle $R$ and $W=0$. Before continuing the measurement two options were available: If a stem was not too tall and measurement was manageable standing upright, then the stem was left attached to the stool. For taller stems however, it was recommendable to horizontally draw a line on the stems at a fixed height of e.g. $100 \mathrm{~mm}$ from the ground. Alongside this line the direction towards the stool's center was then marked with a vertical line. Along the horizontal marking the stem was then cut whilst carefully bringing the stem down without branches or twigs breaking off. The stem was then mounted in a custom built wooden fixture comparable in function to a sawbuck (see panel (c) in Figure 3.4). The branches should not touch the ground or bend in any way due to the fixing. Independently of a stem being measured as a whole still attached to the stool or after being cut down, all growth units were labeled with tags then numbered consecutively, starting with order 0 and then continuing by fully labeling lateral axes and their successors working from stem base to tip. For easier orientation, the scars at 
shoot bases and other important points were marked on the stem (see panel (d) in Figure 3.4. Additionally, a topological layout/sketch was drawn of each tree (see panel (a) in Figure 3.5).
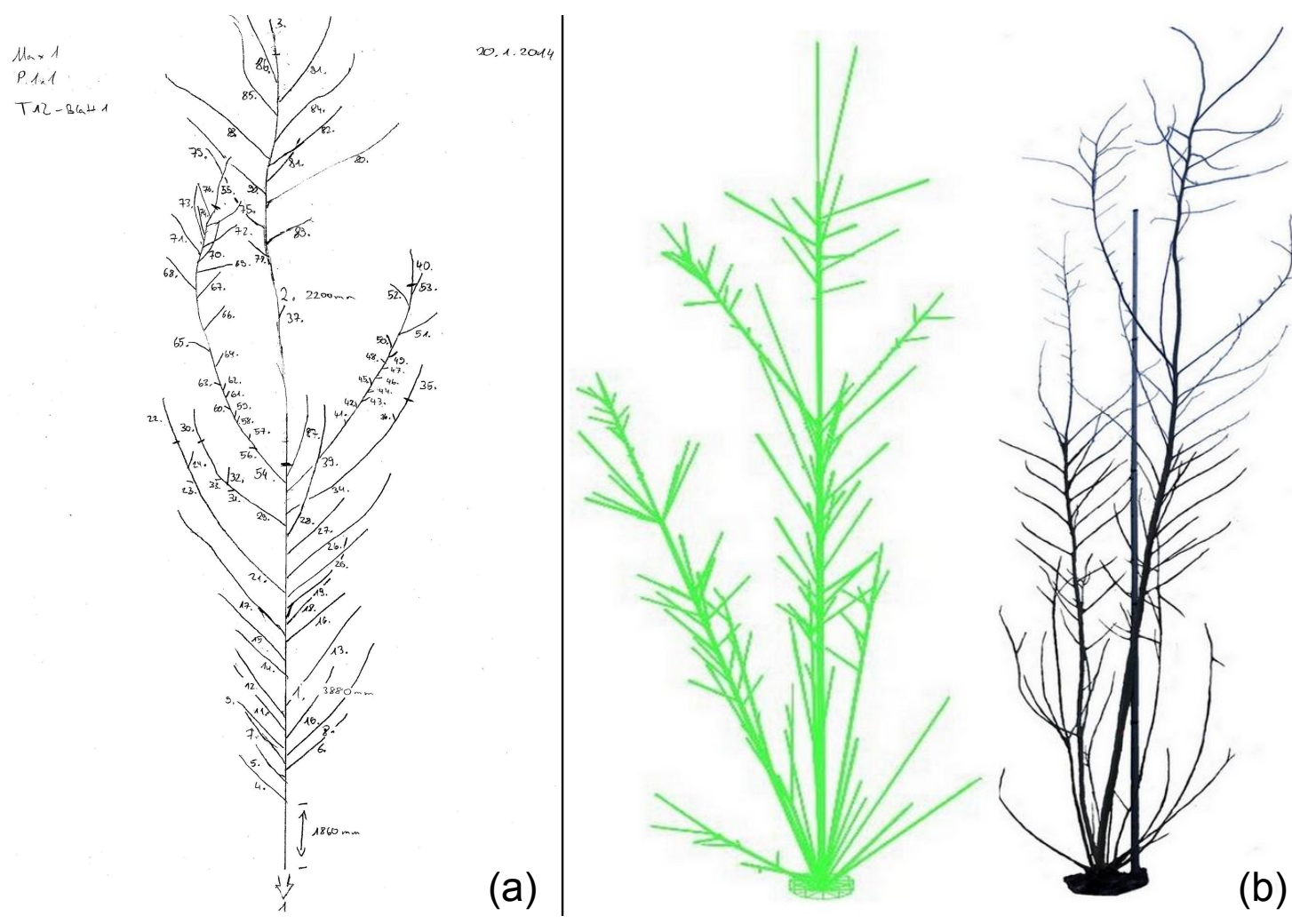

Figure 3.5: Exemplary illustration of different visualization of topology and structure. Panel (a) shows the topological sketch of the middle part of the main stem of a 'Max 1' tree (Sketch drawn by Niels Lakämper). Panel (b) shows a photograph of the same tree on the right and on the left the visualized dtd file from GrolMP (Illustration in panel (b) taken from Lakämper, 2014, p. 11).

All growth units are then measured regarding the variables as specified in Table 3.1. A minimal workforce should consist of 2 persons who divide the measuring itself and the data recording amongst each other. An additional person can be useful to further divide the measuring when multiple tools are needed to avoid switching.

The specific measurement mainly incorporated the same steps as the detailed morphological variant. The measurement itself however differed by recording the length and mid diameter of all GUs only from branch order 0 of all main and minor stems. Additionally the internode count and the count of lateral axes (branch 
order 1) were counted. This procedure was applied to a sample of $n=12$ trees with $n=4$ trees per clone.

The third summary variant of structural data acquisition applied the specific variant only to the major stem while for the minor stems only length and middiameter per GU were measured. A total amount of $n=27$ trees was measured here. The count of trees per group is unbalanced here due to 3 additional trees that were measured for 'Max 1' before the plantation was harvested completely.

A list of all measured trees grouped by the measurement variant is given in Table 3.2 .

Table 3.2: List of trees that were randomly selected for differently detailed DTD measuring during winter 2013/2014 in pl17 Unterrieden.

\begin{tabular}{|c|c|c|}
\hline Clone & Tree Identifier & Type \\
\hline MAX & 1_001,1_2_091,1_3_121,1_4_175 & detail \\
\hline & 2_1_019,2_2_061, 2_3_139, 2_4_181 & detail \\
\hline AF2 & 3_1_043, 3_2_073, 3_3_115, 3_4_175 & detail \\
\hline MAX & 1_1_007, 1_2_073, 1_3_139, 1_4_145 & specific \\
\hline (B & 2_1_025, 2_2_09 & speci \\
\hline r & 3_1_037, 3_2_079, 3_3_109, 3_4_169 & specific \\
\hline MAX & $\begin{array}{l}\text { 1_1_019, 1_1_037, 1_1_043, 1_2_066, } \\
\text { 1_2_080,1_3_115, 1_3_127, 1_3_133, } \\
\text { 1_4_163, 1_4_169, 1_4_181 }\end{array}$ & $\mathrm{y}$ \\
\hline HYB & $\begin{array}{l}\text { 2_1_031, 2_1_037, 2_2_049, 2_2_085, } \\
\text { 2_3_109, 2_3_121, 2_4_163, 2_4_175 }\end{array}$ & mmar \\
\hline F2 & $\begin{array}{l}\text { 3_1_001, 3_1_013, 3_1_031, 3_2_049, } \\
\text { 3_2_054, 3_2_061, 3_3_097, 3_3_121 }\end{array}$ & \\
\hline
\end{tabular}

After finishing the measurement, the data was digitized and then imported into $\mathrm{R}$ for a full plausibility check with validating the data types and value entries. Based on the topology, age and order were computed for all GUs as these were initially documented only for branch order 0 in the field. Furthermore the dtd files were imported into GrolMP for visual checking (see example in panel (b) of Figure 3.5. 


\subsubsection{Branch Curvature}

Evaluating the data from the DTD measurement it became evident that the curvature of stems and branches was not incorporated adequately into the model. A clear bending of the shoots can already be assessed visually (see Figure 3.5). Branch curvature is mentioned by Ceulemans et al. (1990) as an important feature in selecting poplar clones for high density plantations. Therefore curvature was rated as essential for the structural model at hand. Keeping the manual approach, a more realistic representation of the curvature would have necessitated further extensive measurement. The latter implying that the full orientation of single internodes or alternatively shoot segments (as done by Casella and Sinoquet, 2003) should have been quantified. This would have been too time-consuming using the dtd approach. As already established, the half-automatic approach with the electromagnetic digitizer FASTRAK was available whose methodological employment will be described in this section.

\section{Polhemus FASTRAK motion tracking system and Blender Addon}

The whole FASTRAK system as employed for the curvature measurement consists of the following components (see also Figure 3.6).

- System Electronics Unit (SEU) with power supply

- Transmitter

- Stylus receiver

- Wooden tripod

The SEU is the core component. It connects to the transmitter and the receiver, processes their signals and outputs them to the connected computer. The transmitter creates an electro-magnetic field and functions as the reference for a single or multiple receivers (Polhemus, 2012). In this case only a single receiver was used in the form of the stylus device. The stylus has a pen shape and a button for multiple possible actions like triggering measurements. For measuring, the stylus has multiple coils built into its tip. In combination with the electro-magnetic field this enables the system to capture the current 3D coordinates of the tip and 

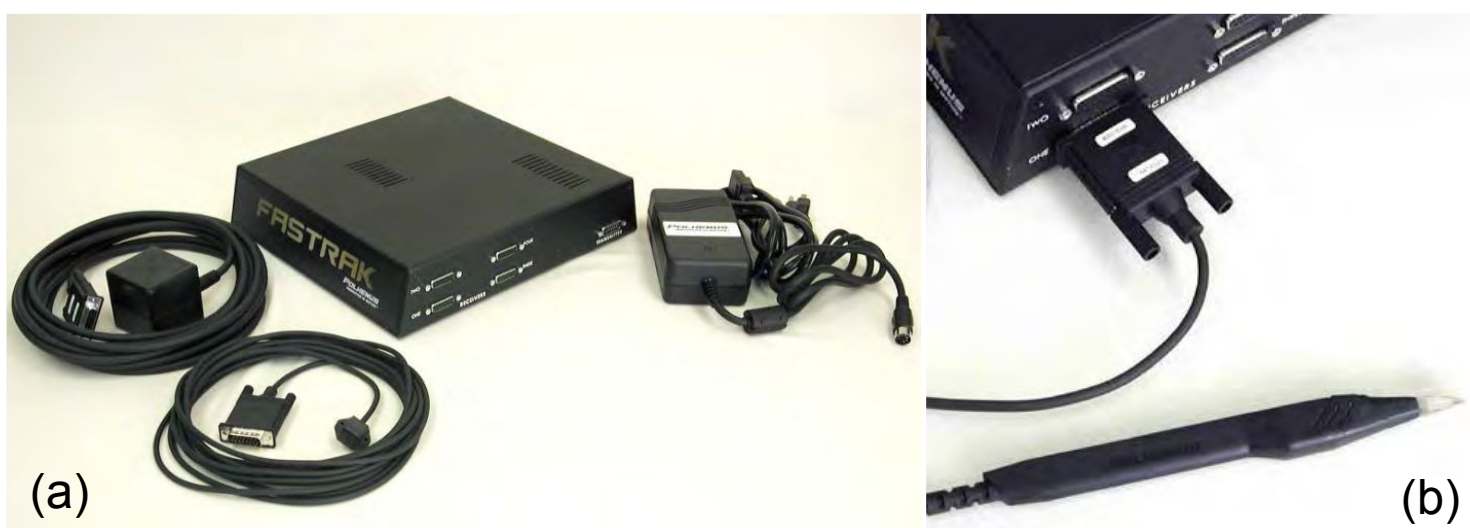

Figure 3.6: Components of the FASTRAK system. In panel (a) the System Electronics unit (SEU; middle), the transmitter (black cube, left), the standard receiver (bottom) and the power supply (right) are shown. In panel (b) the stylus is shown that also works as a receiver (Image source: Polhemus, 2012, (a) p. 1, (b) p. 25).

the orientation of the pen relative to the transmitter position. Because of the offset of the stylus' tip and the coil assembly the SEU uses an algorithm for correction of the coordinates. The detailed functionality is described in Polhemus (2012). The wooden tripod is a purpose-built item for properly positioning the transmitter at a desired height and horizontally aligned (determined by spirit level). As suggested by Polhemus (2012) the tripod lacks any metal components which could disturb the signals. This already points towards the requirements for the measurement environment mentioned in the FASTRAK manual (Polhemus, 2012):

- Any larger amount of metal in the measuring range should be avoided. This can also include walls, floors and ceilings in and outside of buildings.

- The cables of the transmitter and the receiver should not be crossed.

- The operating temperature of the system is between $10{ }^{\circ} \mathrm{C}$ to $40{ }^{\circ} \mathrm{C}$ at a relative humidity of $10 \%$ to $95 \%$ non-condensing.

Operating outside these conditions or ignoring these factors could lead to a distortion in measurement or the system not functioning properly in general. The manufacturer states that under proper conditions an accuracy in the sub-mm range is possible up to a distance of $1 \mathrm{~m}$ around the transmitter (Polhemus, 2012). The recommendation for the specified accuracy however is to operate 
the receivers within $760 \mathrm{~mm}$ of the transmitter if standard devices are used (Polhemus, 2012). It is possible though to use the standard devices with greater distances up to $3050 \mathrm{~mm}$ but with reduced accuracy of up to $40 \mathrm{~mm}$ (Polhemus, 2012). Danjon and Reubens (2008) designate a $1500 \mathrm{~mm}$ radius sphere as the measuring range. Experiences during field work have confirmed this value as sufficient for measuring nodes for branch structure. There are alternative devices like a long ranger transmitter available that can increase the operational distance of the whole system (Polhemus, 2012). This was also successfully utilized by Danjon and Reubens (2008) and Surový et al. (2011) and extended their range to 4 to $5 \mathrm{~m}$. Since the long ranger device was not available, the measurements described here were conducted with standard equipment.

For processing the data coming from the FASTRAK system, multiple software implementations are available. There is a native host software by Polhemus which provides some basic functionalities for measuring 3D coordinates. Due to the fact that this program was not specifically designed for measuring plant morphology, it lacks some desired properties. There were 3 available software solutions considered that were implemented with this specific goal:

- PiafDigit (Donès et al., 2006) as cited and used by Danjon and Reubens (2008), Scheinost (2012) and Chambelland et al. (2008) for example

- FastrakDigitizer (FORMATH Research Group, 2013) used by Surový et al. (2011) and Yoshimoto et al. (2014)

- An addon for the 3D modeling software Blender (Blender Foundation, 2012) by Wasilczuk (2012)

Trivially, these implementations have their advantages and disadvantages. Regarding PiafDigit, Wasilczuk (2012) declares that the user interface in the French language as well as problems with memory management and handling exceptions are problematic. After trying the 2 nd and 3rd option, the decision was made to work with the solution by Wasilczuk (2012) since this seemed to work best for the task at hand.

The addon has two operating modes: continuous and discrete. In the continuous mode, points are recorded continuously based on a customizable threshold 
distance which the stylus has to be moved. This mode works best for measuring shapes like leaf margins. The discrete mode only records single coordinates once the stylus' switch button is pressed. This works best for measuring the position of nodes and therefore their internodes and the structure of the shoot that these form. For graphical representation of measured coordinates, multiple geometric forms like cylinders and spheres can be chosen in the addon. These forms are further customizable by their size and shape. Spheres were chosen for the measurement here as they visually seemed most appropriate for distinguishing nodes. Besides the latter, other plant organs like leaves, flowers and fruits can be specified for measurement. These options were unneeded in this case. When measuring nodes, these can be assigned to different plants and shoots, meaning that they get different identifiers within the addon. Further functionalities of the addon include a calibration to prevent any possible, unwanted systematic bias in measuring the points. Specifying a geographical direction and a possible slope of the ground is also possible.

After the measurement of each shoot, topological information can be added with the addon. This can then be exported in the MTG file format (Godin et al., 1997; Pradal and Cokelaer, 2009) although the implementation here is rather basic. Therefore it was decided to also separately record the topological information manually. Besides saving the measurements in the blender file format, the 3D coordinates and their essential identifier data can be exported as csv files.

A full manual on how to install the addon and the comprehensive documentation is given in Wasilczuk (2012).

\section{Measuring methodology and procedure}

Measuring was carried out from the end of March to the beginning of April in 2017. An overall number of 15 trees were measured with 5 trees per clone. A list of all trees is given in Table 3.3 . 
Table 3.3: Trees that were randomly selected for branch curvature measurement in fw11 Stiedenrode I during March 2017.

\begin{tabular}{lrrr}
\hline Clone & Plot & Row & \multicolumn{1}{c}{ Tree } \\
\hline MAX & 25 & 2 & $3,5,6,7$ \\
MAX & 25 & 4 & 6 \\
HYB & 22 & 2 & $3,4,5,6$ \\
HYB & 22 & 3 & 8 \\
AF2 & 76 & 2 & $6,8,9$ \\
AF2 & 76 & 3 & 6,10 \\
\hline
\end{tabular}

The utilized equipment included the FASTRAK system, the wooden tripod, a laptop, a power generator as well as the measuring and marker tools that were already part of the manual data acquisition. The mensuration took place outside and in setting up the working environment, special attention was given to keep any metal objects or electronic devices away from the FASTRAK transmitter and receiver. Therefore the calibration function of the addon was not really needed, the procedure nevertheless proved useful to determine that all components worked properly before starting to measure.

Measuring the trees required some preparations since the height of the trees and the limited range of the FASTRAK digitizer posed a conflict at first. Building some sort of pedestal or using a ladder was considered as not practicable. Furthermore any constructions should not contain too much metal which made an improvised lightweight solution difficult. The decision was made to measure the trees in several steps as it was the case for the dtd manual structure measurements. First each stool per selected tree was measured within the stand. If possible, multiple stools were measured at once. Close to the stools, the transmitter mounted on the tripod was positioned with a compass so that the marked $x$ direction on the transponder was aligned with the northern cardinal point. The tripod was then leveled horizontally with a spirit level. On the main stem of each tree, instead of measuring all internodes, it was opted for measuring 4 points equidistant on each side of the stem in $500 \mathrm{~mm}$ intervals to a height of $1500 \mathrm{~mm}$. Minor stems were fully measured in the stand if they did not exceed a height of $1500 \mathrm{~mm}$. On all stems taller than this, 8 calibration points were marked on the bark and their position was also recorded. The stems were then cut by handsaw 
below the 8 calibration points. Stems were transported upright out of the stand and carefully mounted in a custom built wooden fixture as it was the case for the manual dtd measurement (see also Figure 3.7). Careful handling of the stems is mandatory to prevent the breaking of dried branches or small twigs.

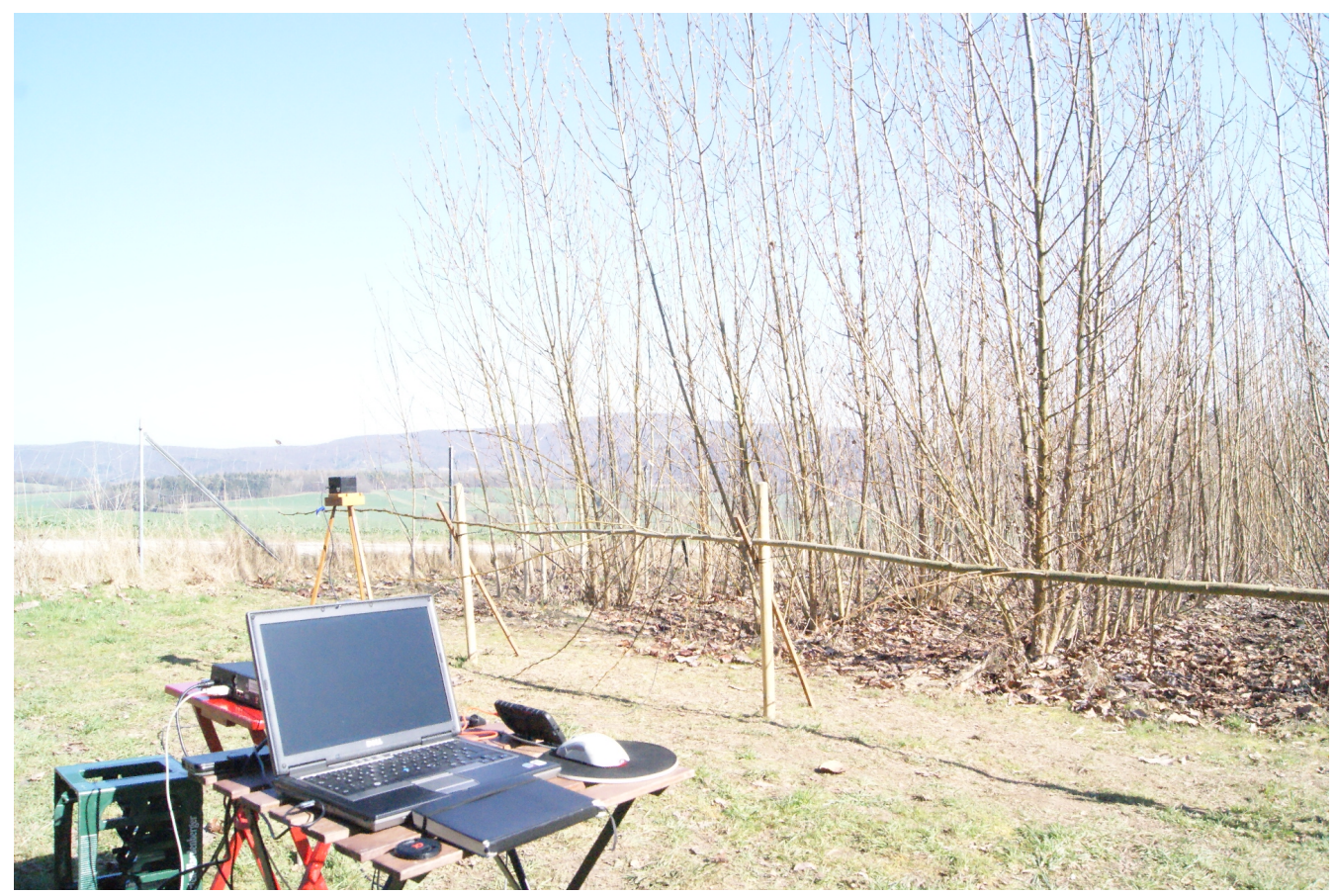

Figure 3.7: Example for the setup of measuring branch curvature along the main stem of a 'Hybride 275' tree.

The FASTRAK transmitter was then repositioned so that ideally the lower third of the tree could be measured. The 8 calibration points were measured again to allow for a connection of the separate parts. The remaining part of the main stem was then measured in $50 \mathrm{~mm}$ intervals per growth unit. Lateral branches (branch order $>0$ ) and minor stems (branch order $=0$ ) were measured at the shoot base with 4 equidistant points. From the shoot base to the tip all nodes were measured with the FASTRAK stylus as points. The shoot tip (omitting the bud) was marked as well. The general direction of working was always from the trees' base along the main stem towards the top. Based on the insertion height, branches and their daughter growth units (lateral or prolongation) were then measured fully. If the cut off tree still exceeded the transmitter range in length, additional calibration points were added and recorded. The transmitter was then repositioned, calibration points measured again, and the whole measurement procedure could be 
continued in the upper part of the tree. While data acquisition proceeded faster as with the dtd method, measuring all nodes for all shoots remains very labor intensive and time-consuming. Hence every second branch was skipped for detailed assessment. The reduced intensity only included measuring the four base points, the tip and any lateral daughter branches if present.

\section{Data processing}

During and after the field measurements, the 3D point data was checked for consistency and plausibility. Few shoots had to be discarded if the tip had broken off or the tip coordinates were found to be too biased afterwards. The coordinates were then exported as csv files. These were then imported into $\mathrm{R}$ for further processing. The data had to be reformatted for analysis to follow the conventions of Wickham (2014). Since all trees were measured in multiple parts, the next step included joining the separate datasets. Following the terminology of Dryden and Mardia (2016) multiple ordinary Procrustes analyses were conducted for this purpose. Ordinary implies that one observation was matched onto another. Observation here included only the 8 calibration points per part as a $8 \times 3$ matrix. Joining two parts is a question of rotating and translating the to be transformed matrix to a point where the sum of squared Euclidean distances between original and to be transformed calibration points is minimized. This necessitates a least squares matching procedure comparable to the one common in linear regression for example.

For two configurations $X_{1}^{0}$ and $X_{2}^{0}$ and then matching $X_{1}$ to $X_{2}$, through Procrustes analysis the result is the fitted configuration $X_{1}^{P}$ (example taken from Dryden and Mardia (2016, p. 145). The root mean square deviation (RMSD) then is:

$$
R M S D=\sqrt{\frac{1}{k}\left\|X_{2}-X_{1}^{p}\right\|^{2}}
$$

With $k$ being the number of points (Equation adapted from Dryden and Mardia, 2016, p. 145). This formula also contains the ordinary Procrustes sum of squares $(O S S)$ as $\left\|X_{2}-X_{1}^{p}\right\|^{2}$. Both $R M S D$ and $O S S$ can be used as a measure for assessing how well the calibration points could be matched. For joining all 
separate parts an overall count of 39 Procrustes analyses was performed. All joining procedures were assessed visually and by $R M S D$ and $O S S$. In 2 cases slight manual adjustments by translating and rotating the matched coordinates were necessary because the calibration points were placed too close together.

After joining the datasets, the coordinates were grouped and summarized per tree, stem and growth unit. The combinations of these identifier variables were then output to separate files and the topological information was added. This information was then re-imported into R. Based on the full topology as well as branch order and age information for some GUs, the missing branch order and age information was completed. Additionally, an identifier was added to GUs that form an axis (mother with all prolongation GUs) since these also form a unit from a biomechanical view.

The next act was to quantify the curvature of all growth units. Instead of approximating the curvature in 3D space it was decided to project the node coordinates into $2 \mathrm{D}$ space to be able to access the huge variety of $2 \mathrm{D}$ fitting procedures. The Principal Component Analysis (PCA) was chosen as the means for 3D to 2D projection. The PCA was only applied to the fully measured growth units with coordinates for all nodes. As a preparation for the PCA, the mean was calculated for the 4 points at the shoot base to get the shoot's base center. The PCA itself was applied per GU or, if prolongation units were existent, per axis. The prcomp from the stats package (R Core Team, 2019) was utilized for this. The original 3D point matrix was then multiplied with a matrix that consists of the first two columns of the rotation matrix. The latter is the matrix of variable loadings meaning the columns contain the eigenvectors ( $R$ Core Team, 2019). The first two columns of the rotation matrix are the first two principal components or the two eigenvectors with the largest and second largest eigenvalue $\lambda_{1}$ respectively $\lambda_{2}$. The matrix multiplication then resulted in a 2 column Matrix with the desired $2 \mathrm{D}$ coordinates of the nodes. This matrix was then translated and rotated so that the shoot's base is at the origin and the shoot tip aligns with the $x$-axis. The point of maximum width should have a positive $y$-coordinate meaning that the branch bends into quadrant I. The results of this procedure were plotted, checked and then merged into a single dataset. Branches were projected into $2 \mathrm{D}$ as one and then separated as growth units whose points were then translated and rotated properly. 
After attaining the 2D coordinates, simple shape parameters per $\mathrm{GU}$ were calculated to allow for first assessment of the curvature. Following Stoyan and Stoyan (1994), this included a length ratio like the elongation factor. This basically is the ratio $\frac{b}{l}$ of the width $b$ measured orthogonally to the chord length $l$ as the length from the branch's base to its tip (usually the most extreme points of the growth unit). A second ratio $\frac{l_{m}}{l}$ is calculated for the length $l_{m}$ from the base to the horizontal position of $b$ and the chord length $l$. These ratios were calculated using the raw $2 \mathrm{D}$ node coordinates.

Since the nodes were measured as points on the surface, directly assessing the curvature by calculating the angles between node coordinates leads to a bias because the nodes are arranged in an alternating way on the surface. Considering the internode as a vector between two nodes disregards the internode's thickness. Therefore the inner course of the pith of each GU had to be approximated. Multiple approaches like smoothing algorithms, spline interpolation or fitting Bezier curves were considered. These usually demand some optimizing effort to find a curve that does not fit the data too well but is still flexible enough to represent the curvature well. After visually assessing this for the raw data, a simpler approach of fitting second-degree polynomials proved to be a good compromise between goodness of fit and simplicity. These were then parameterized as linear models. For all models an $F$-test was conducted to compare the fit to a null model. If the $F$-test led to the decision that the second-degree polynomial did not fit the data better than the null model, the growth unit was assumed to be straight and not curved. This was again visually checked and led to a satisfying discrimination of curved and straight growth units. With the predicted $y$ values from the polynomial fit the start and end points of all internodes per GU were calculated. The differences in angle between succeeding internodes were then obtained. These were then used as a response for further modeling. The final goal was to find a function that can predict the differences in angle between succeeding internodes based on GU variables. Figure 3.8 contains an exemplary overview of the whole curvature fitting procedure. 
(a)

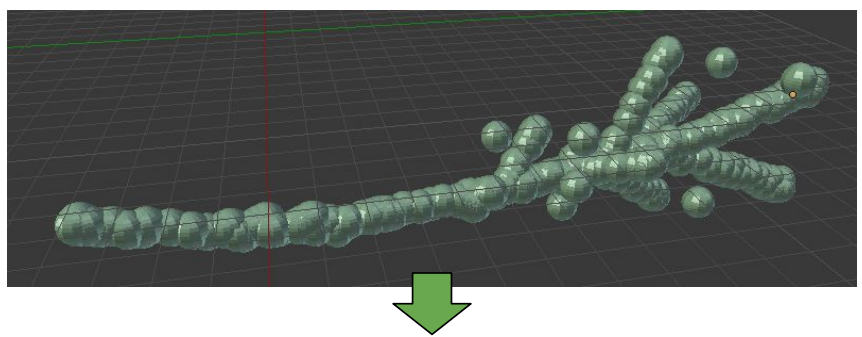

(b)
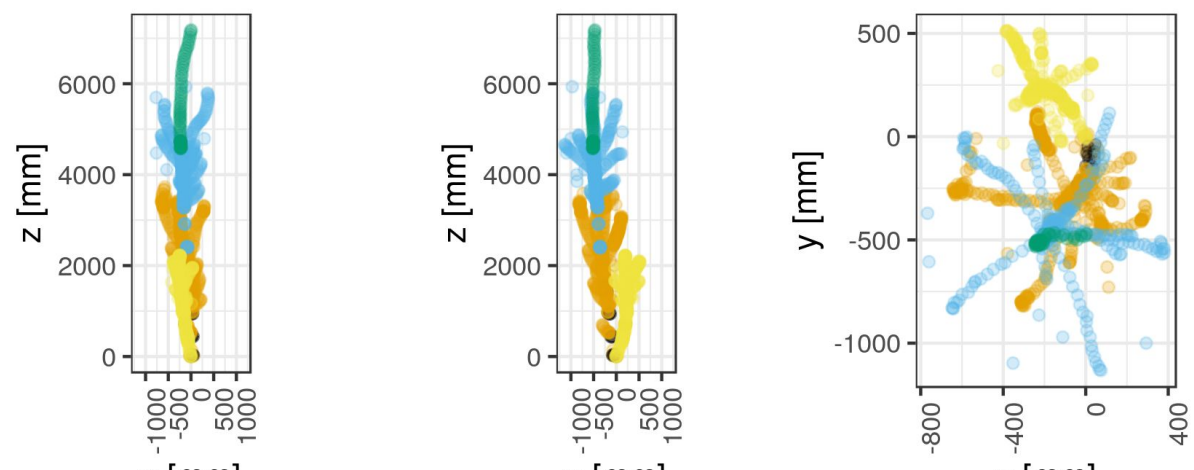

(c)
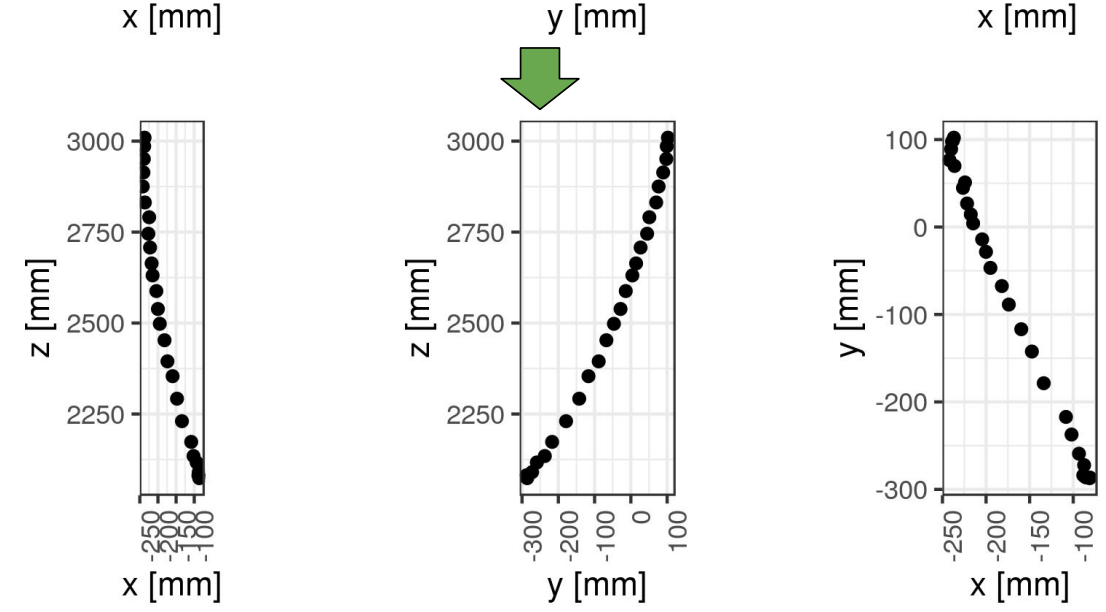

(d)

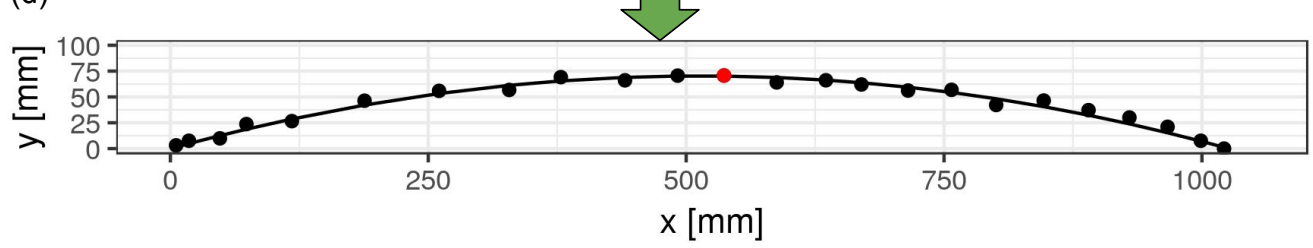

Figure 3.8: Schematized display of the curvature fitting workflow. Panel (a) shows the nodes of a cut off minor stem in Blender after measurement. Panel (b) shows 3 perspectives of the joined parts of the tree ('Max 1') that this minor stem is from. The different colors resemble different parts, i.e. the steps of measurement. The minor stem from panel (a) is colored yellow. Panel (c) shows 3 perspectives of a single growth unit. The black dots mark the nodes. Panel (d) shows the same growth unit after projection from 3D to 2D via PCA. The black line is based on the second-degree polynomial fit. The node marked red has the coordinates $\left(l_{m}, b\right)$. The chord length $l$ here is $1021 \mathrm{~mm}$. 


\subsubsection{Leaf Position and Structure}

Leaf position and structure were studied by measuring leaves from all 3 poplar clones during August 2016 at the FastWOOD trial site fw11 Stiedenrode I. The above-ground biomass, which consists of stem and crown biomass that has grown from resprouting after harvesting 3 years ago, was aged 1 to 3 years at this time. The below-ground biomass, which has basically grown since the year of planting, was 1 to 6 years old. All in all 5 trees were selected at random from 4 plots (see table 3.4.

Table 3.4: Trees that were randomly selected for leaf structure measurement in fw11 Stiedenrode I during August 2016.

\begin{tabular}{lrrr}
\hline Clone & Plot & Row & Tree \\
\hline MAX & 71 & 4 & 6 \\
MAX & 71 & 4 & 8 \\
HYB & 22 & 4 & 6 \\
HYB & 77 & 4 & 7 \\
AF2 & 76 & 4 & 5 \\
\hline
\end{tabular}

The measurement itself consists of three steps: First measuring the tree as a whole, second measuring the branching structure of the stems per tree and third measuring the leaf structure of single leaves per branch. The first 2 steps were conducted in the same manner as devised for the structural measurement in leafless condition (see list in section 3.1.3). The detailed measuring of branching structure and structure of single leaves could not be conducted with the trees still standing since they had reached heights of 6 to $8 \mathrm{~m}$. Therefore the examined stems were cut before measuring. An additional difficulty that arose from this practice was that stems and their branches usually had to be discarded if the measuring was not completed after one day at the latest because the leaves would start to wilt and lose their stability leading to distorted results. Due to the described wilting issue, watering the shoots was an additional step to delay any unwanted effects from withering. These were decreased further by protecting the cut off stems and branches from direct sunlight and wind. The latter was also important for establishing calm conditions that prevent the leaf blades from moving too heavily in the wind which can basically make measuring impossible. Small gaps in the plantation stand can serve as suitable places for measuring 
after separating the stems and their branches. Figure 3.9 contains an exemplary setup for measuring which can also be established in stand gaps.

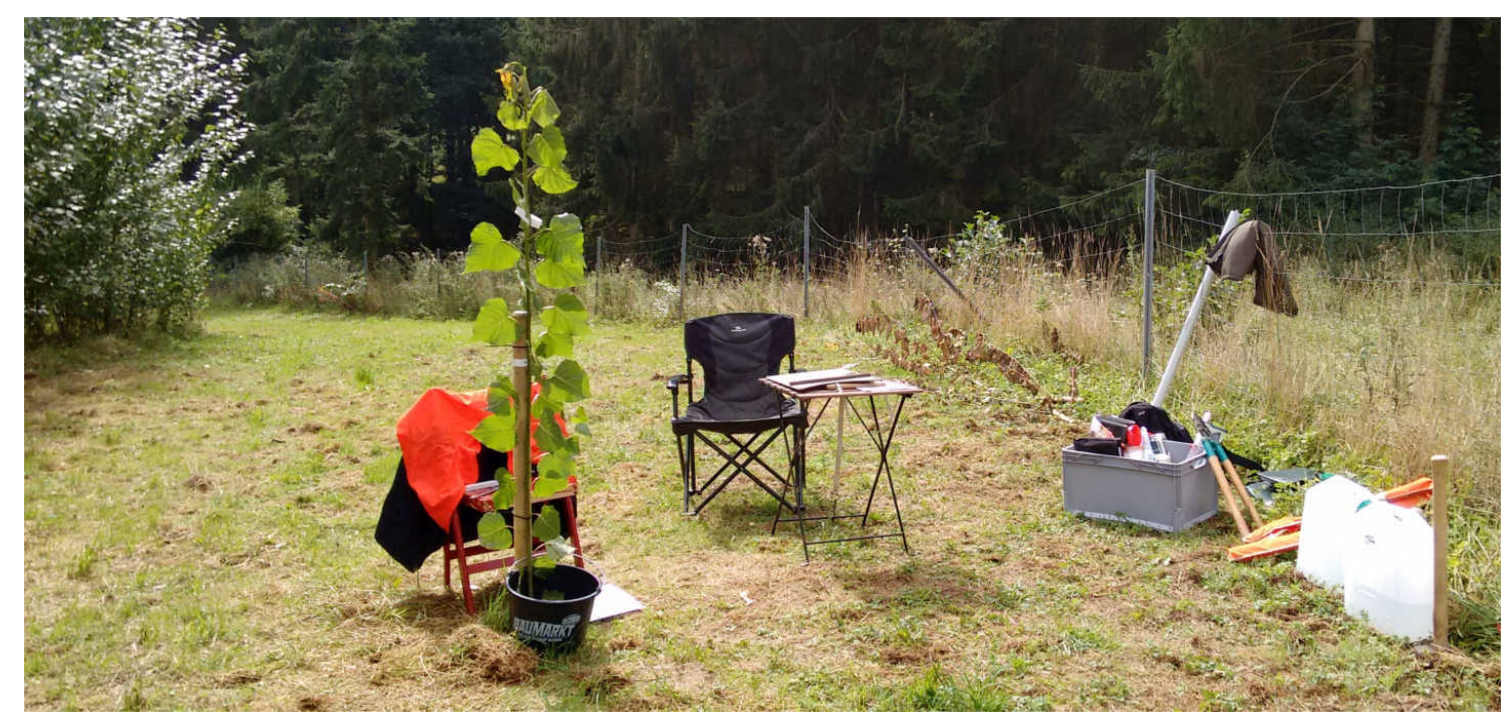

Figure 3.9: Exemplary setup for measuring leaf structure. Shown here is the 3rd growth unit from the main stem of an 'AF2' tree. The shoot was mounted to a pole while being watered at the shoot base (cutting surface). The measurement here is conducted outside of the plantation stand.

After establishing a proper mensuration setup the leaf structure was recorded with an adapted methodology based on the dtd format. A basis for the adaptation is given e.g. in Kurth (2014). The parameters that were measured at the single leaf level are listed in table 3.5 . 
Table 3.5: All parameters that where measured for leaf structure in 2016 on a single leaf level. Values for variables petiR and petiBladR are given as classified angles (see Figure 3.2). The parameters from the associated structural measurements in 2016 conform with the dtd specifications.

\begin{tabular}{lll}
\hline \multicolumn{1}{c}{ Variable } & \multicolumn{1}{c}{ Description } & Unit \\
\hline mother & Identifier of mother growth unit & \\
nodeLeaf & Binary variable: count of nodes carrying leaves (1) versus no leaves $(0)$ & \\
leaf Rank & Leaf rank as leaf number from base & {$[\mathrm{mm}]$} \\
iNodeL & Internode length & {$[\%]$} \\
heightRel & Relative height of leaf in reference to maximum tree height & {$[\%]$} \\
relPos & Relative position in reference to growth unit length & {$[\mathrm{mm}]$} \\
petiL & Petiole length & {$[\mathrm{mm}]$} \\
petiD & Petiole diameter & {$\left[{ }^{\circ}\right]$} \\
petiW & Orientation angle between internode and petiole & {$\left[{ }^{\circ}\right]$} \\
petiR & Directional angle of petiole to shoot in regard to reference direction & {$\left[{ }^{\circ}\right]$} \\
petiBladW & Angle between leaf blade and petiole & {$\left[{ }^{\circ}\right]$} \\
petiBladR & Angle of the rotation of the leaf blade in reference to the petiole & {$[\mathrm{mm}]$} \\
bladL & Length of leaf blade & {$[\mathrm{mm}]$} \\
bladW & Width of leaf blade & \\
\hline
\end{tabular}

For easier understanding of the meaning of each variable, Figure 3.10 contains a display of all variables shown for example leaves and their mother growth units. 


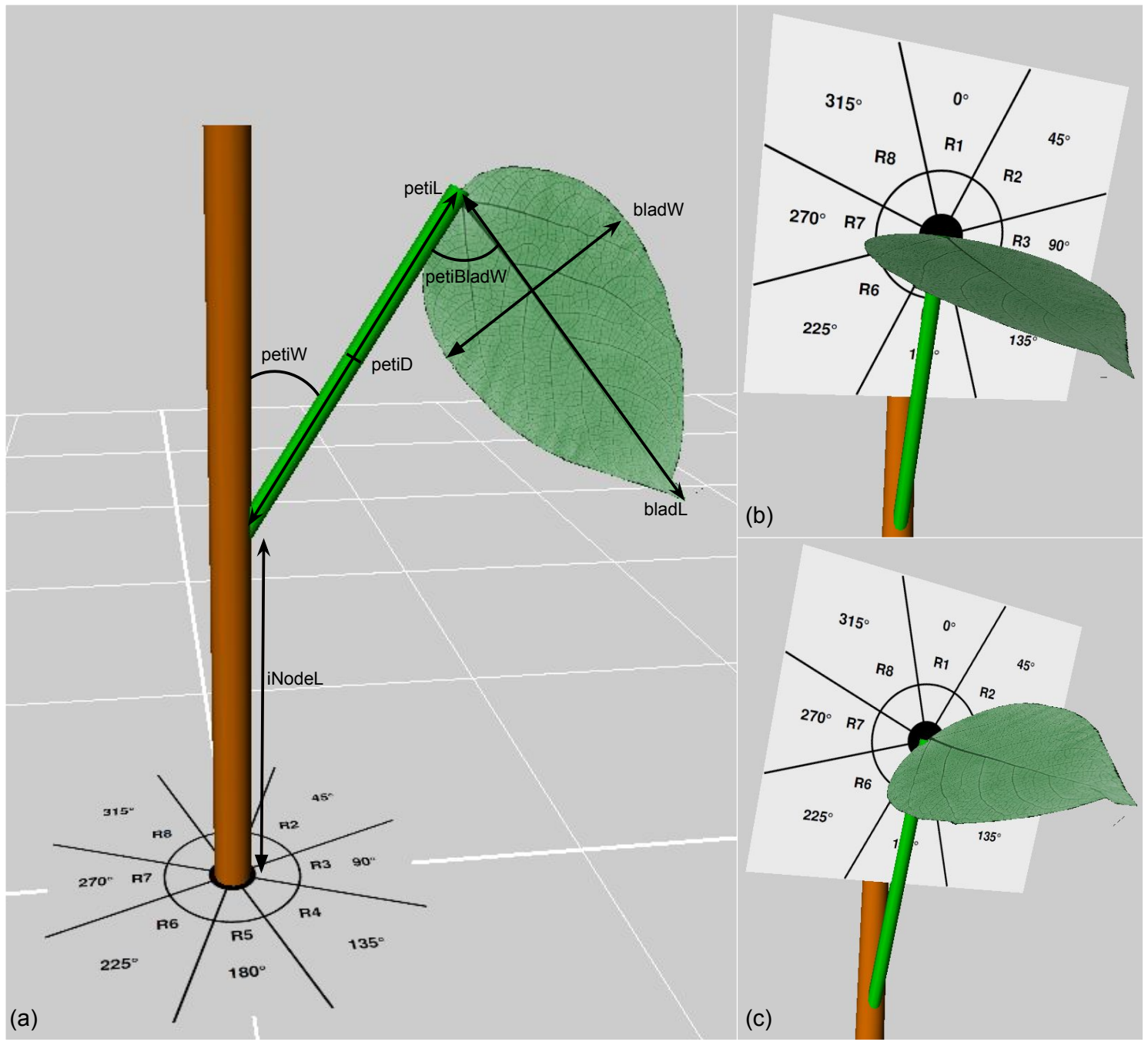

Figure 3.10: Explanatory display for better understanding of variables measured with the dtd format adapted to leaf shape. Part (a) shows most of the variables described in Table 3.5. Variable petiR and its value is not marked but can be acquired from the directional schema at the bottom of the GU. The value is R3 here. Parts (b) and (c) exemplify different values for parameter petiBladR: R3-7 for (b) and R2-6 for (c). 
While measuring the leaf structure the occurrence of leaf whorls could be observed, meaning that more than one leaf was present per node. A majority of these whorls was observed on growth units from the last vegetation period. On some of these growth units of age 2, single leaves were also observed. An example is given in Figure 3.11. Panel (a) illustrates a comparably high frequency of what appears to be leaf whorls on growth unit 2 ( 2 years old, order 0 ) while the upper growth units carry mostly single leaves. This separation of more alleged leaf whorls in the lower crown and more single leaves in the upper crown can be supported with the images in panels (b) and (c).

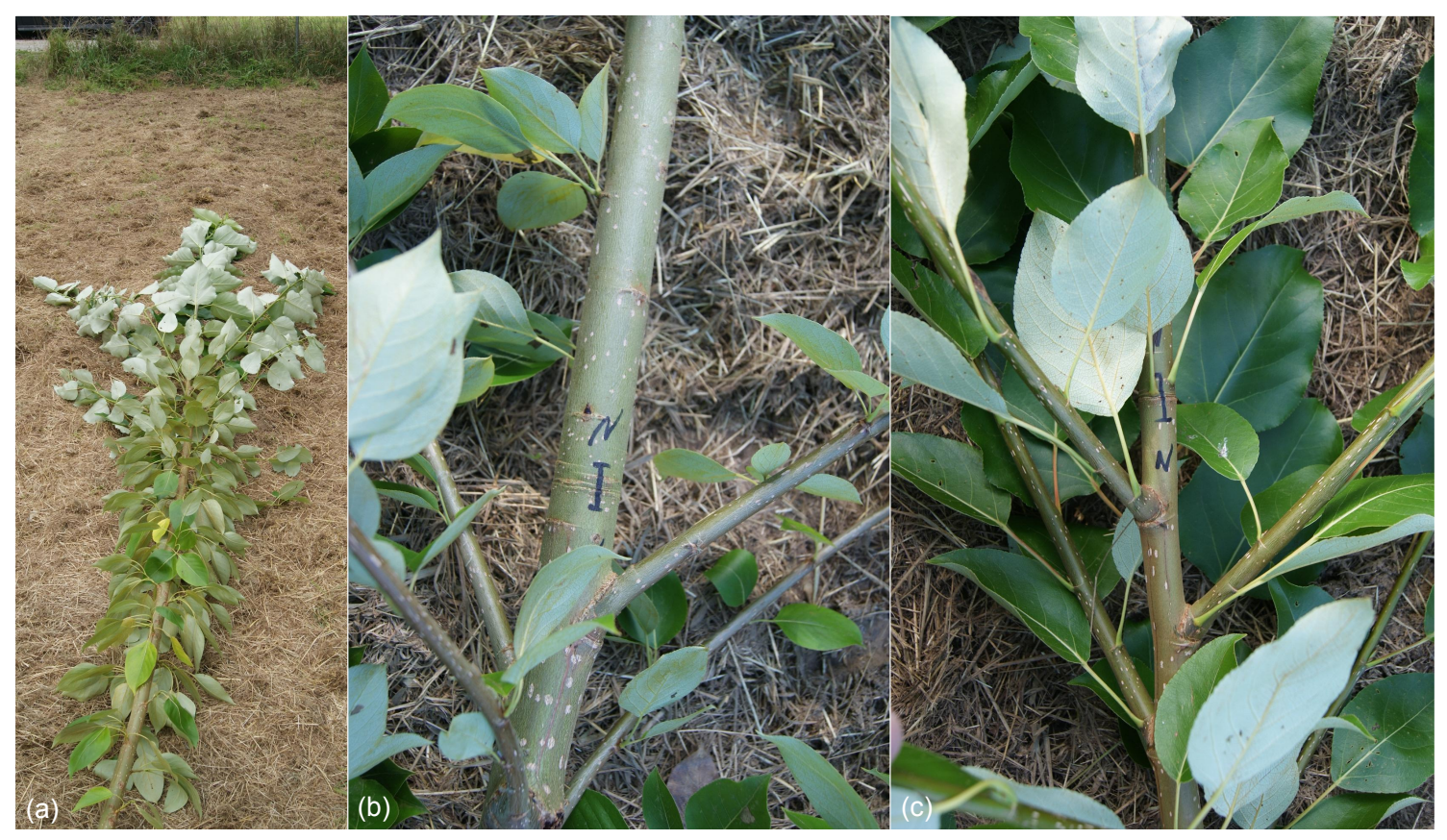

Figure 3.11: Example of occurrence of single leaves and what appears to be leaf whorls. All 3 photographs were taken from 'Hybride 275' trees. The left panel (a) shows the top of the main stem of tree 77_4.7. The middle panel (b) shows the border between growth unit 1 (age 3, order 0 ) and 2 (age 2, order 0 ) of the main stem of tree 22_4.6. The panel on the right (c) shows the border between growth unit 2 (age 2, order 0 ) and 3 (age 1, order 0 ) of the same main stem of tree 22_4.6. The border is marked with a black line facing north in both cases.

Due to the position of 2 year old growth units, these leaves, either single or in whorls, were mainly observed in the lower half of the tree. At first these leaves were recorded in the same manner as leaves on growth units that had developed in the current vegetation period. For leaves in whorls, an additional identifier 
was given to record whorl affiliation. The orientation of the whorl itself and the orientation of the leaves within a whorl was documented. By closer investigation and discussion of the results it became obvious that single leaves and whorls that were originally recorded with a 2 year old mother growth unit were actually growing on short shoots with very short internodes (compare also Critchfield, 1960; Dickmann et al., 2002). These short shoots hence had developed during the current growing season. To give a botanically sound representation of this background, short shoots were added to the structural data that inherited some of their properties from the initially recorded leaf position like insertion height and orientation. These short shoots were then set as the mother growth unit of the respective leaves.

Due to the overall high detail of the whole manual measuring technique, which inherently implies a comparably high time consumption, not all branches and leaves of the selected trees were measured. Only every 3rd growth unit was fully measured with all leaves. For the "non-detailed" GUs only the branching architecture, the number of single leaves and leaves per short shoots were recorded. For the "detailed" GUs the leaves on every 4th node including single leaves and all leaves on a short shoot where fully measured with all parameters from Table 3.5 . Leaves at the shoot tip were always measured even if their leaf rank didn't fall into the measuring grid. For the remaining leaves only the $\mathrm{iNodeL}$, petiR, bladL and bladW values were recorded.

After acquisition all data was digitized and imported into $\mathrm{R}$. The data was controlled with some plausibility checks and then prepared for further processing. The structural data that was acquired was transformed into the dtd format and then imported into GrolMP. GrolMP offers some of the Grogra functionalities including listing all shoots and their $x$-, $y$-, and $z$-coordinates. The information can be outputted in several formats like txt or csv. This can then be reimported into $R$ for calculating the height of leaves and growth units. The calculation could be implemented in $\mathrm{R}$ as well, but it seemed more convenient to use the already available GrolMP features.

Regarding the utilized statistical procedures and methodology as well as the $R$ packages see section 3.1.8. 


\subsubsection{Leaf Shape}

During September 2017 an amount of 91 leaves were collected from all 3 clones at the FastWOOD trial site fw15 Stiedenrode II. Above-ground biomass in stems and crowns was aged 1 to 2 years at this time while the below-ground root biomass was 1 to 5 years old. Each tree was separated into 3 equally sized sections in regard to tree height. From each section 10 leaves with petiole and lamina were collected randomly. One additional leaf was collected for AF2 hence $n=91$. These leaves were then mounted on paper and dried in a plant press. After drying, the leaves were placed on black velvet as a background and digitized via the scanning function of a photocopier. Leaves were scanned from their bottom side because the contrast to the black velvet seemed better. Additionally a sheet of millimeter paper was scanned and digitized for determining the scale of $1 \mathrm{~mm}$ being 24 pixels. The images of the leaves only required minor post-processing (cropping and rotating) which was done within the image processing software GIMP (Kimball et al., 2017). By applying the ImageJ (Rasband and Team, 2017) macro from Henke et al. (2014) leaf contours were extracted. These output text files contain $x$ - and $y$-coordinates of the 2D leaf border as well as leaf size summarising parameters like leaf blade (lamina) width, length and stalk (petiole) diameter. The coordinates and the size parameters were imported separately into $\mathrm{R}$ for data processing and analysis. The $2 \mathrm{D}$ shape coordinates were outputted with the manually chosen leaf base at the origin, the main leaf vein oriented along the $y$-axis and the leaf tip pointing in the positive direction of the $y$-axis. This orientation of the coordinates required further processing. In accordance with Henke et al. (2014) the leaves were separated along the direction of the main leaf vein into two halves, left and right. As the point for separation the coordinate with the maximum $y$ value was chosen. Whilst both halves have their own set of points, they both share the leaf tip coordinates. The denomination for both leaf halves was corrected for the upper sides of the leaves since the bottom side was scanned. Leaf halves were then computed separately. By determining the angle between the vector to the tip coordinates and the $x$-axis, a rotation matrix was formed that would rotate the coordinates clockwise so that the tip would lay on the $x$-axis with the $y$ coordinate of the tip equaling 0 . Additionally the coordinates of the right leaf half were then mirrored along the $x$-axis for better comparability of both leaf halves. 
Figure 3.12 shows an example of the single steps from digitizing to preparing for analysis.

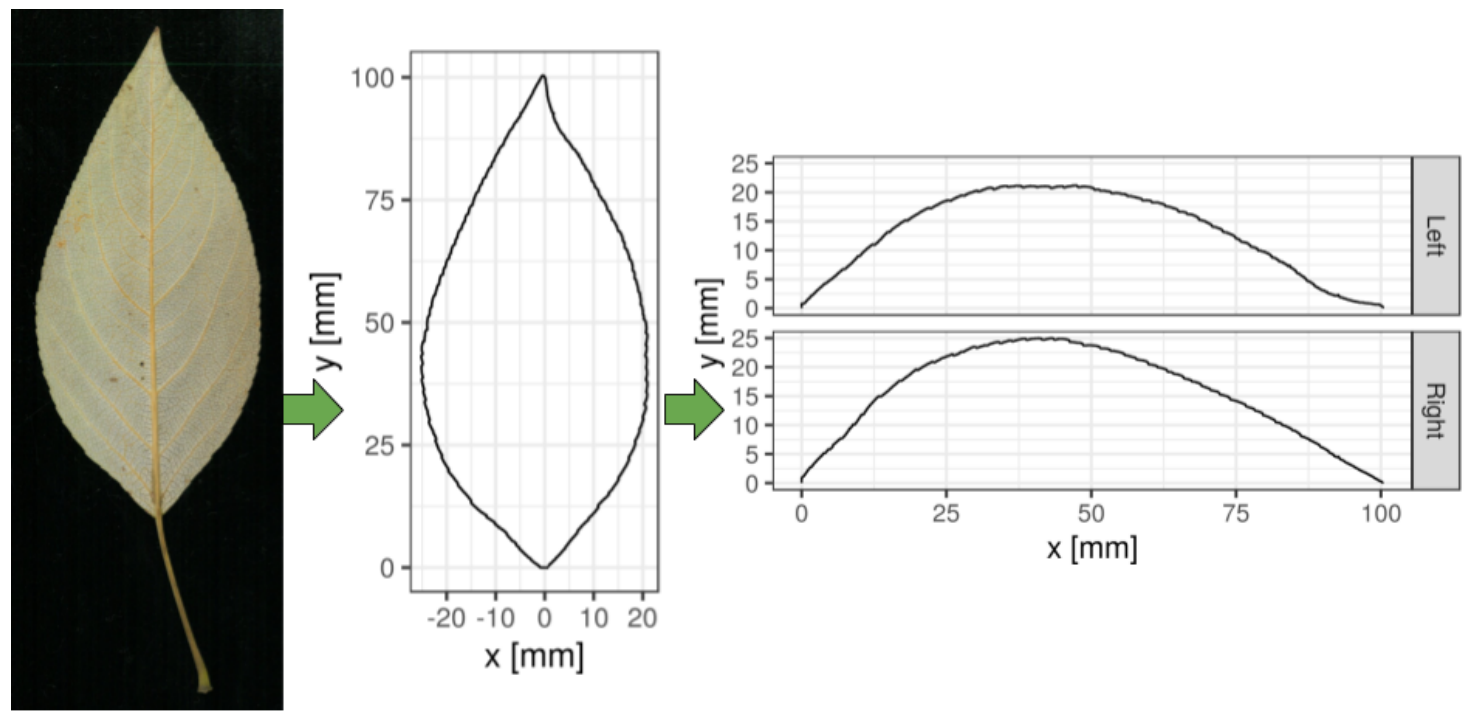

Figure 3.12: Steps in leaf shape digitizing from images of scanned leaves (left) to digitized shape imported into $\mathrm{R}$ (middle) and separated, rotated leaf halves (right). Shown here is a leaf from the middle section of a 'Hybride 275' tree.

Since the leaf shape was measured at one point in time and with no temporal repetition, only the static model approaches by Henke et al. (2014) were considered for shape modeling. This mainly comprehends the proportional shape model based on ratios of shape parameters. The polynomial approach was not feasible for the studied clones here. Due to the cordate leaf base morphology of especially larger leaves, these do not exhibit the desirable property that the contour of the leaf halves can be viewed as a function of vertical leaf blade height as it was the case for the Populus $\times$ canadensis leaves from Henke et al. (2014). This is further evaluated in section 3.2.10. For the proportional shape model by Henke et al. (2014) 3 support/contour points are needed. It soon became evident that this number was not sufficient to reflect the leaf half contours of the probed clones here. 
To improve this, 6 additional support points were determined as follows, ordered by increasing $x$-coordinate:

- $S_{1}=\left(0,0.5 * d_{\text {petiole }}\right)$

- with $d_{\text {petiole }}$ : diameter of leaf petiole

- $S_{2}=\left(S_{2_{x}}, 0.2 * b\right)$

- with $b$ : maximum vertical width perpendicular to $\mathrm{x}$-axis of leaf blade

- $S_{3}=\left(S_{3_{x}}, 0.5 * b\right)$

- $S_{4}=\left(S_{4_{x}}, 0.8 * b\right)$

- $S_{5}=\left(l_{m}, b\right)$

- with $l_{m}$ : horizontal position of said maximum vertical width

- $S_{6}=\left(S_{6_{x}}, 0.8 * b\right)$

- $S_{7}=\left(S_{7_{x}}, 0.5 * b\right)$

- $S_{8}=\left(S_{8_{x}}, 0.2 * b\right)$

- $S_{9}=(l, 0)$

- with $l$ : maximum leaf blade length

Apart from the support point at the leaf basis $S_{1}$, at the widest vertical expansion of the leaf blade $S_{5}$ and the leaf tip $\left(S_{9}\right)$, extra support points were added at the vertical positions of 20,50 and $80 \%$ of $b$ in the lower and upper part of the leaf half. Figure 3.13 exemplifies the placement of all support points along the contour of a leaf half. 


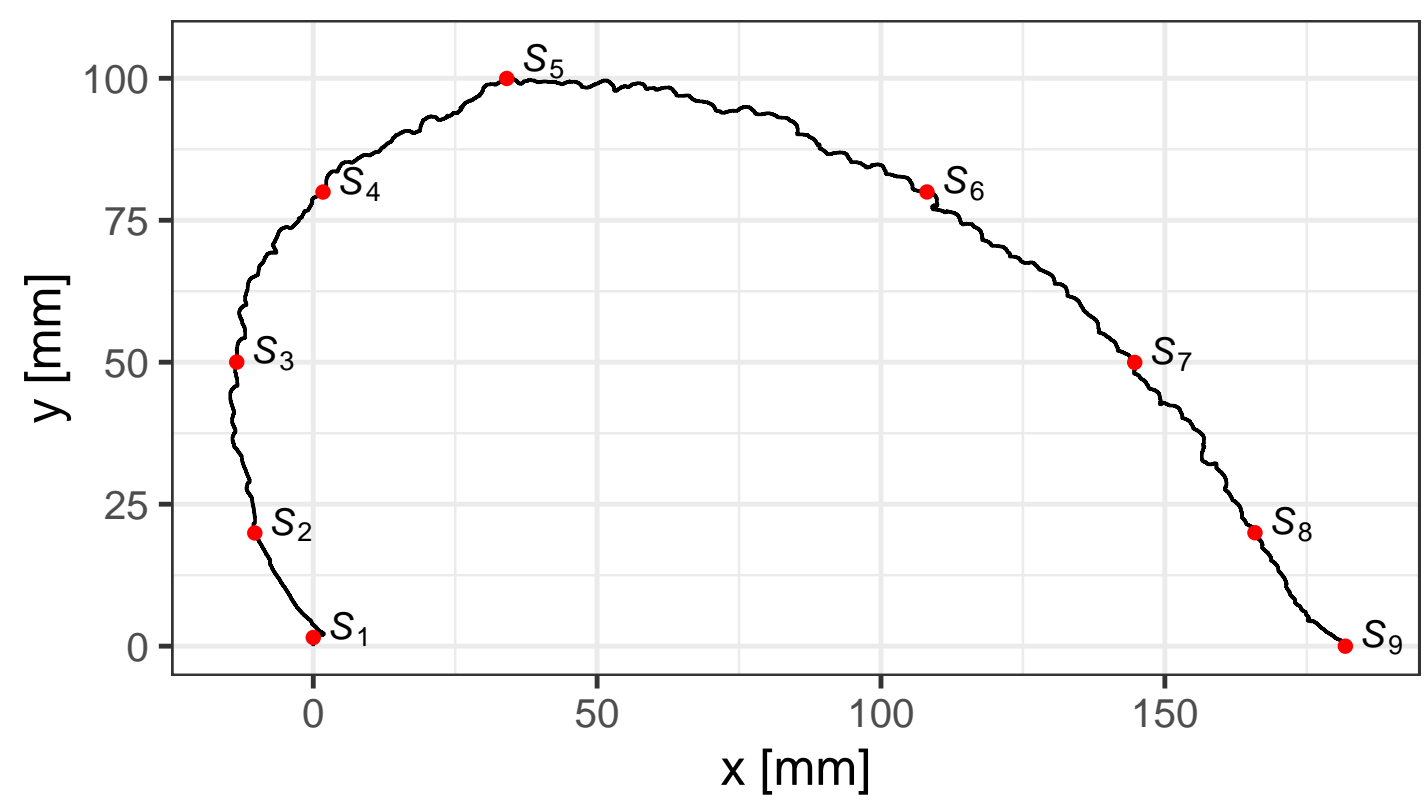

Figure 3.13: Example for the placement of the 9 support points along the contour of a leaf half of a leaf from the upper section of a 'Max 1' tree. The depicted leaf half also illustrates the cordate leaf base shape.

For modeling leaf shape the following procedure was deemed practicable:

1. Estimate leaf blade length $l$ or respectively $b l a d L$ with the leaf structure models described in sections 3.1.5 and 3.2.9.

2. Estimate $b$ and $d_{\text {petiole }}$ from $l$.

3. Estimate $x$-coordinates of remaining contour points.

All to be estimated coordinates were incorporated into ratios in accordance to Henke et al. (2014). Their approach involved the shape parameters as the nominator and the leaf blade length $l$ as the denominator for all ratios and then estimating these ratios by length, revealing the latter as a good predictor of shape. This approach had to be refined since it became evident early that due to the cordate leaf base shape of some clones, the predictive ability of some models was rather poor. After some attempts the ratios for the lower part of each leaf half were modified resulting in all ratios as follows: $\frac{S_{1_{y}}}{l}, \frac{l_{m}-S_{2_{x}}}{l}, \frac{l_{m}-S_{3_{x}}}{l}, \frac{l_{m}-S_{4_{x}}}{l}, \frac{l_{m}}{l}, \frac{b}{l}$, $\frac{S_{6_{x}}}{l}, \frac{S_{7_{x}}}{l}$ and $\frac{S_{8_{x}}}{l}$. 
For all given ratios linear models by the following form were parameterized:

$$
y=\beta_{0}+\beta_{1} x+\beta_{2} z+\beta_{3} x z+\epsilon
$$

where $y$ is the response ergo the respective ratio, $\beta_{0}$ is the intercept, $x$ is the quantitative covariate leaf blade length with coefficient $\beta_{1}, z$ is the qualitative covariate clone with $\beta_{2}$ as its coeffcient whereas $x z$ is the interaction term of leaf length and clone with coefficient $\beta_{3} . \epsilon$ is the random error. The full model was parameterized for each ratio, the models were checked if any assumptions were violated. Via the procedure for stepwise model selection by BIC, variables were tested and selected for remaining in the model while assessing the model's predictive ability. This resulted in the parameterization of 9 regression models (estimation of $S_{9}$ not counted). While the estimation of the contour points via length was now possible and ready for implementation in $\mathrm{XL}$, further processing was needed to create curves from these points that approximate the leaf contour. Again, the approach by Henke et al. (2014) of calculating a parametric curve $C(s)$ was advanced. The $x$ - and $y$-values of said curve were interpolated separately by two Hermite interpolations. As with Henke et al. (2014) these interpolations $s p 1(s)$ and $s p 2(s)$ were between the 9 previously estimated contour points. Henke et al. (2014) used 3 points. The parameter $u_{2}$ now also includes the added contour points (see Equation 3.3).

$$
u_{2}=\sqrt{\left(\sum_{i=1}^{9} S_{i_{x}}\right)^{2}+\left(\sum_{i=1}^{9} S_{i_{y}}\right)^{2}}
$$

The definition of the final curve $C(s)$ is again the same as in Henke et al. (2014), with $s \in\left[0, u_{2}\right]$

$$
C(s)=(s p 1(s), s p 2(s))
$$


In addition to this expanded proportional shape model other approaches were also considered:

- Estimation of contour $x$ - and $y$-coordinates by their relative position along the contour length (basically the arc length).

- Circumventing the fact that the contours' $y$-coordinates of leaves with a cordate leaf shape base morphology cannot be seen as a function of $x$ by further bisecting leaf halves. This could be done along a straight line that passes through the point $\left(l_{m}, b\right)$ and is perpendicular to the $x$-axis. These newly formed quarters then could be rotated so that the function property is satisfied.

- Fitting Bézier curves to the contour coordinates then analysing the Bézier curves' control points and checking if their coordinates can be estimated from size parameters like $l$ or $b$.

These three approaches were tested but did not yield acceptable approximations of the leaf shape or were not as parsimonious in comparison to the expanded proportional model. Hence these results are not further evaluated here.

\subsubsection{Statistical Modeling and Model Procedure}

If possible the data from different measurements were combined for statistical analysis. This was applied predominantly to the structural information gathered through the manual dtd measurement, the semi-automatic FASTRAK digitizing and the structural data acquired together with leaf structure and position. Because the scale of the information gathered by manual measuring was the growth unit, the positions of empty nodes were estimated with the function for distribution of internode lengths from the contact digitizing data (see Table 3.17). This procedure was necessary to correctly resemble the probability of a lateral branch developing by its relative position on the mother. No branch/orientation or directional angles were calculated for the FASTRAK data. Some tryouts were made with projecting mother and daughter shoots onto a 2D plane identified by principal component analysis and calculating the angle between two respective linear fits. This did not yield satisfying results that were consistent with the already manually 
measured values. As a temporary solution, the directional angles of the minor axes and the branch angles of third order growth units were manually obtained from the digitized point data. This was taken up to increase the already small sample size of the dtd data in these categories.

Based on the findings and the model by Plazas Cebrian (2014) the modeling procedure was outlined before data analysis and the formulation and parameterization of the statistical models was oriented towards the layout of the structural model.

The basic principles of statistical model fitting and variable selection are the same as described for the yield model (see Section 2.1.6).

\subsubsection{Software}

For the statistical analysis the same software and packages were used as already established in Section 2.1.6. The negative binomial distribution was fitted via the function provided through the MASS package (Venables and Ripley, 2010, version 7.3-51.4). An additional package was the shapes package (Dryden, 2018, version 1.2.4) for the Procrustes analysis. The leaf area within the leaf shape modeling was calculated with the function for calculating the area of polygons by the sp package (Bivand et al., 2013, version 1.3-1). The Bézier curves for the leaf contours were fitted with the bezier package (Olsen, 2018, version 1.1.2). For operating the FASTRAK digitizer through the addon by Wasilczuk (2012) the Blender software (Blender Foundation, 2012) was used. The structural model was implemented in GrolMP (GrolMP Developer Team, 2016). For the random number generators used in the structural model the Stochastic Simulation in Java library (short: SSJ L'Ecuyer, 2018) was integrated as a plugin into GroIMP. An installation guide for the SSJ library can be obtained together with the source code for the structural model as explained in the Appendix. The SSJ library was chosen for its variety in different distributions for random number generators. Overdispersion (in relation to a standard Poisson-glm) was often detected for the count data models which was accommodated for by using the quasipoiss on model family or the negative binomial distribution. It turned out to be problematic that the SSJ random number generator with an underlying negative binomial distribution yielded values outside of the observed range. While this, theoretically, does not have to 
be incorrect it leads to problems with other models which use the output as independent variables. Since the glm with the negative binomial model family also estimates the mean of the count data on the given independent variable data it was decided to input the mean counts into a Poisson random number generator. This reduces the variability in the values output by the stochastic functions and is more of a temporary solution. The better way to proceed here would be to find further factors that explain the remaining variability and overdispersion. This would require further processing of the data or measuring more parameters in the future. This will be elaborated further in the discussion (see Section 5).

\subsubsection{Interconnection of Yield Model and Structural Model}

Following the results for the structural model, the implementation of the interconnection of yield and structural model will be described (see Section 4). The general approach was to first fully implement the yield and the structural model and then consider the requirements for their interconnection. This strategy was employed to ensure that both models were developed without any further restrictions, adaptations should be provided by the interconnection. An already exsiting interface implementation between TreeGrOSS/BWINPro and GrolMP has been put into realization in the framework of a student project (Oberländer, 2019). This interconnection works as a GrolMP plugin that generates $\mathrm{XL}$ code based on the $\mathrm{XML}$ files that are output by BWINPro. The plugin is currently being integrated into GrolMP.

For connecting the yield and structural model at hand, however, the decision was made to implement the interconnection directly within the structural model. Only minor adaptations to the structural model had to be made in this case and two additional statistical models were necessary. The latter requirement is based on the fact that the yield model mainly relies on estimating the height and height increment in [m] while the elementary model units of the structural model are the internodes and length is estimated based on internode count. Since it was deemed unfeasible to change the focus of any of the two models, the height values output by the yield model are transformed into internode counts. Furthermore, within the structural model the growth of the minor stems is not related to the growth of the main stem so far. The parameterization of the model is based 
on the data acquired on three locations. Since the focus of the yield model is broader in terms of environmental conditions the growth of the minor stems has to be adapted as well otherwise they would outgrow the main stem if the growth potential of the simulated site is lower. Therefore a model was parameterized based on the ProLoc data to quantify the relation of main stem and minor stem length. The interconnection was implemented in its own RGG project and will be made available together with the structural model. Some additional libraries were necessary for the XML import. 


\subsection{Results}

\subsubsection{Stool Architecture}

The first step in modeling the tree architecture after cutting is determining the number of resprouting stems per stool. Based on the trees measured for the branch structure, leaf structure and branch curvature, the number of stems per tree was summarized. For a total amount of 39 observations, the mean is 8.82 stems, the median 9, the standard deviation 4.31 and the variance respectively 18.6. The tree-wise number of stems was used as a response for a generalized linear model. The differences in scale between mean and variance already point towards overdispersion. Using the negative binomial model family improved the fit over the quasi-Poisson approach with dispersion parameter $\Phi=2.11$. For variable selection, only the variable clone was tested which did not yield a significant effect. Therefore a simple mean model was chosen. The deviance of the null model (which is also the residual deviance in this case) is 40.69 on 38 degrees of freedom. The link parameter was estimated with $k=7.46$ with a standard error of 3.25. The $D_{a d j}^{2}$ parameter is not meaningful for a null/mean model.

The $n$ stems were then separated into 1 main stem and $n-1$ minor stems. As stated in section 3.1.3, the main stem is assumed to be orthogonal to the ground which is considered to be the $x y$-plane here. For the minor stems however, orientation $(W)$ and directional $(R)$ angles were recorded. Per tree, the directional angles of the first growth units of each minor stem were sorted in ascending order. The difference in directional angle between consecutive axes, also known as the divergence angle, was then calculated. Since these values were recorded in 8 sectors (see Figure 3.2) the class midpoints were subtracted for consecutive observations. For each tree the mean value of these differences $\Delta R$ was calculated and these means were used as a response for a linear model. Understandably, the count of stems was suspected to have an influence on this response because observations in the field point towards an even distribution of stem's directional angles per stool. This relationship is displayed in Figure 3.14 which confirms the visual assessment. 


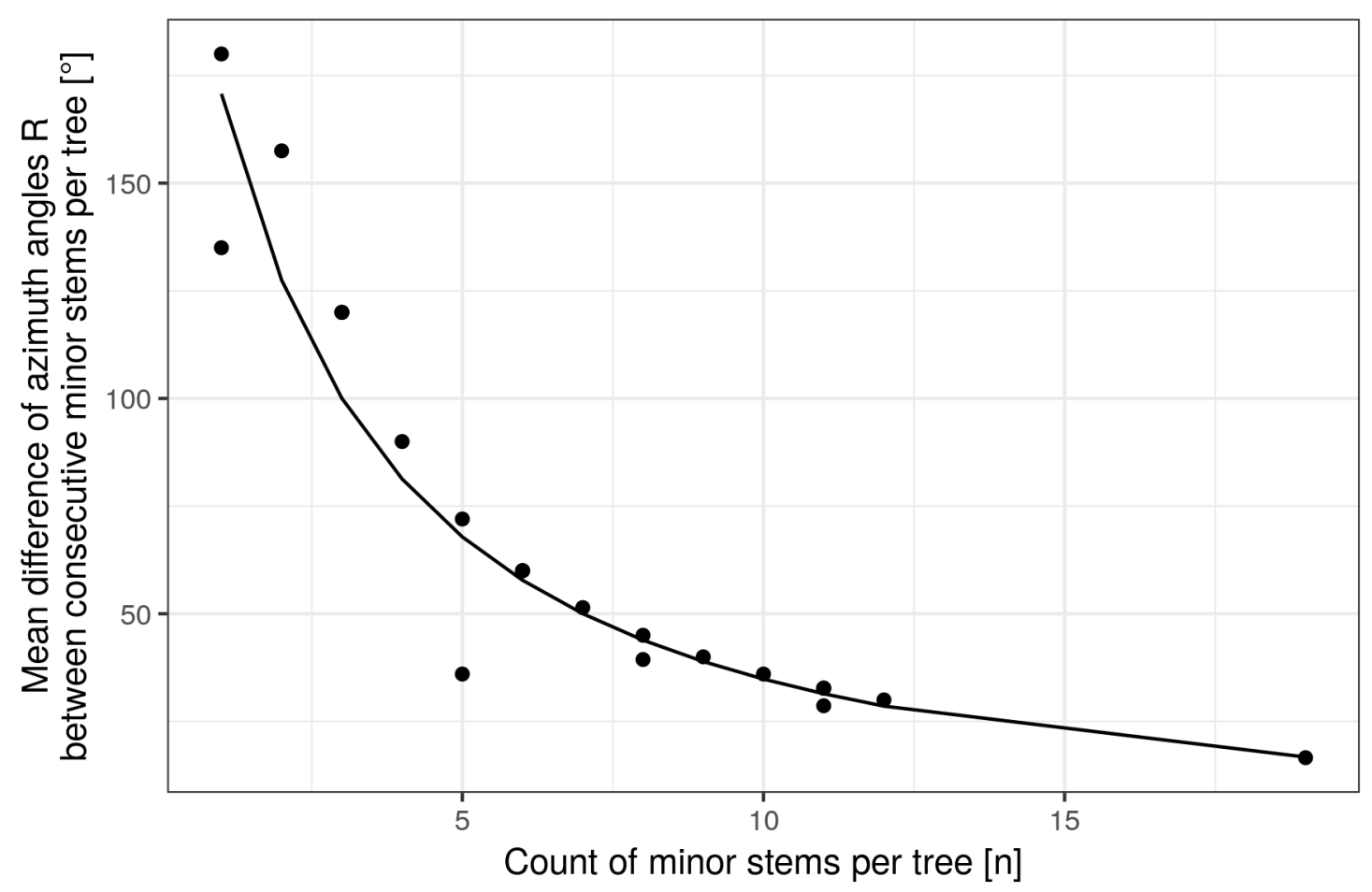

Figure 3.14: Relationship of mean differences between consecutive minor stems' directional angles $R$ and the count of minor stems, both per tree. The black line resembles the back-transformed estimations from the linear model. 
The mean difference or mean divergence angle decreases with increasing number of minor stems. The variable clone was tested as a covariate but no significant influence on the response could be detected. The model diagnostics are given in Table 3.6 .

Table 3.6: Summary of linear model fit for estimating the mean differences in consecutive stem's directional angles stemR. The response was transformed as stem $R^{-0.7}$. Independent variable is the number of stems per stool $n$.

\begin{tabular}{ccrrrrrr}
\hline$\hat{\sigma}$ & $R S S_{\text {Null }}$ & $d f_{\text {Null }}$ & $R S S_{\text {Model }}$ & $d f_{\text {Model }}$ & $F$ & $p$ \\
\hline 0.00721 & 0.01704 & 22 & 0.00109 & 21 & 306.5 & $<0.001$ \\
\hline & \multicolumn{7}{c}{10} \\
& Covariate & Est. & se & $t$ & $p$ \\
\cline { 2 - 7 } & Intercept & 0.02116 & 0.00292 & 7.3 & $<0.001$ \\
& $n$ & 0.00621 & 0.00035 & 17.5 & $<0.001$ \\
\hline
\end{tabular}

As determined with the Box-Cox procedure, the response was transformed by raising it to a power of -0.7 to meet the linear model assumptions. The predictive power evaluable by $R_{a d j}^{2}=0.93$ is very high and the good approximation of the response can be confirmed in Figure 3.14 (black line based on back-transformed model function).

Figure 3.15 contains the correlation of the length of each minor stem's first GU and its orientation angle $W$. Apparently there is a decrease in the angle $W$ with increasing GU length, implying longer GUs to be more upright similar to the main stem.

Again, this relationship was modeled by linear regression with the orientation angle $W$ as the response. The latter had to be square-root transformed. The significant covariates are the first GU's length $l$ and the factor clone (see model diagnostics in Table 3.7). 


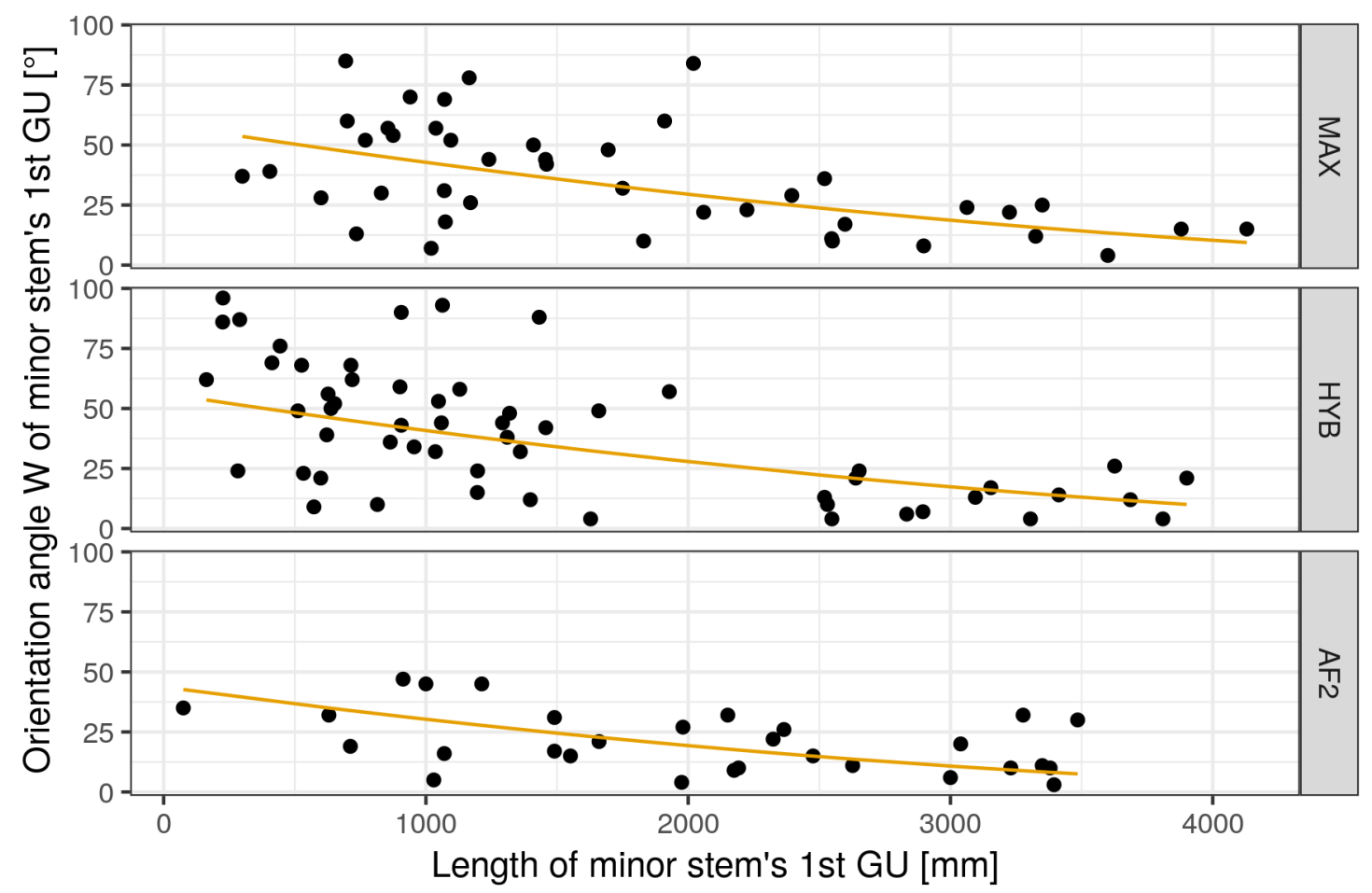

Figure 3.15: Relationship of each minor stem's first GU's orientation angle $W$ and its length $l$, separated by clone. The black lines resemble the back-transformed estimations from the linear model.

Table 3.7: Summary of linear model fit for estimating the stem's orientation angle stemW. The response was transformed as $\sqrt{\text { stemW. }}$. Independent variables are the length of the first GU of each stem $l$ as well as clonal affiliation clone.

\begin{tabular}{crrrrrc}
\hline$\hat{\sigma}$ & $R S S_{\text {Null }}$ & $d f_{\text {Null }}$ & $R S S_{\text {Model }}$ & $d f_{\text {Model }}$ & $F$ & $p$ \\
\hline 1.62 & 540.34 & 127 & 326.41 & 124 & 306.5 & $<0.001$ \\
\hline & \multicolumn{7}{c}{} \\
& Covariate & Est. & $s e$ & $t$ & $p$ \\
\cline { 2 - 7 } & Intercept & 7.65183 & 0.34811 & 22.0 & $<0.001$ \\
& $l$ & -0.00111 & 0.00014 & -8.0 & $<0.001$ \\
& cloneHYB & -0.15410 & 0.33113 & -0.5 & 0.642 \\
& cloneAF2 & -1.03858 & 0.39190 & -2.7 & 0.009 \\
\hline
\end{tabular}


The interaction term of length and clone did not turn out to be significant and was therefore dropped as a covariate. Judging from Figure 3.15 and the model coefficients, the influence of variable clone seems rather low in comparison to length $l$. Clone 'Hybride 275' does not significantly differ from 'Max 1' while 'AF2' does. The negative coefficient points towards smaller angles for 'AF2' which concurs with the observed slender habitus of 'AF2'. It poses a difficulty for the model that less observations were made for 'AF2' overall and especially in the lower length range (see Figure 3.15). The value 0.38 for the adjusted coefficient of determination $R_{a d j}^{2}$ can be seen as intermediate.

\subsubsection{Shoot Growth}

For determining if a bud will break dormancy and a shoot is going to develop, several cases were distinguished based on the bud's position:

- Buds located on the stool

- Terminal buds

- Lateral buds

In the framework of the model described here, on the stool, only buds are considered that will develop shoots. The probabilities for terminal and lateral buds to develop shoots are modeled separately by two generalized linear models. Both models focus solely on the probability of regular shoot growth. The possibility of developing sylleptic shoots is examined with an additional model. For terminal buds, the structural data was subsetted so that only growth units were considered that could have developed a prolongation growth unit within the observed rotation cycle which basically are growth units with age $>1$. The GUs from the main stem with branch order 0 were also excluded since it was assumed that they would always form a prolongation shoot. For the lateral buds, the data was filtered in the same manner so that only nodes were included that could have developed a regular lateral shoot during the 3 vegetation periods. Figure 3.16 shows the distribution of GU length separated by whether the terminal bud of this GU has developed a prolongation shoot or not. The different facets in the graph relate to the clonal affiliation. 


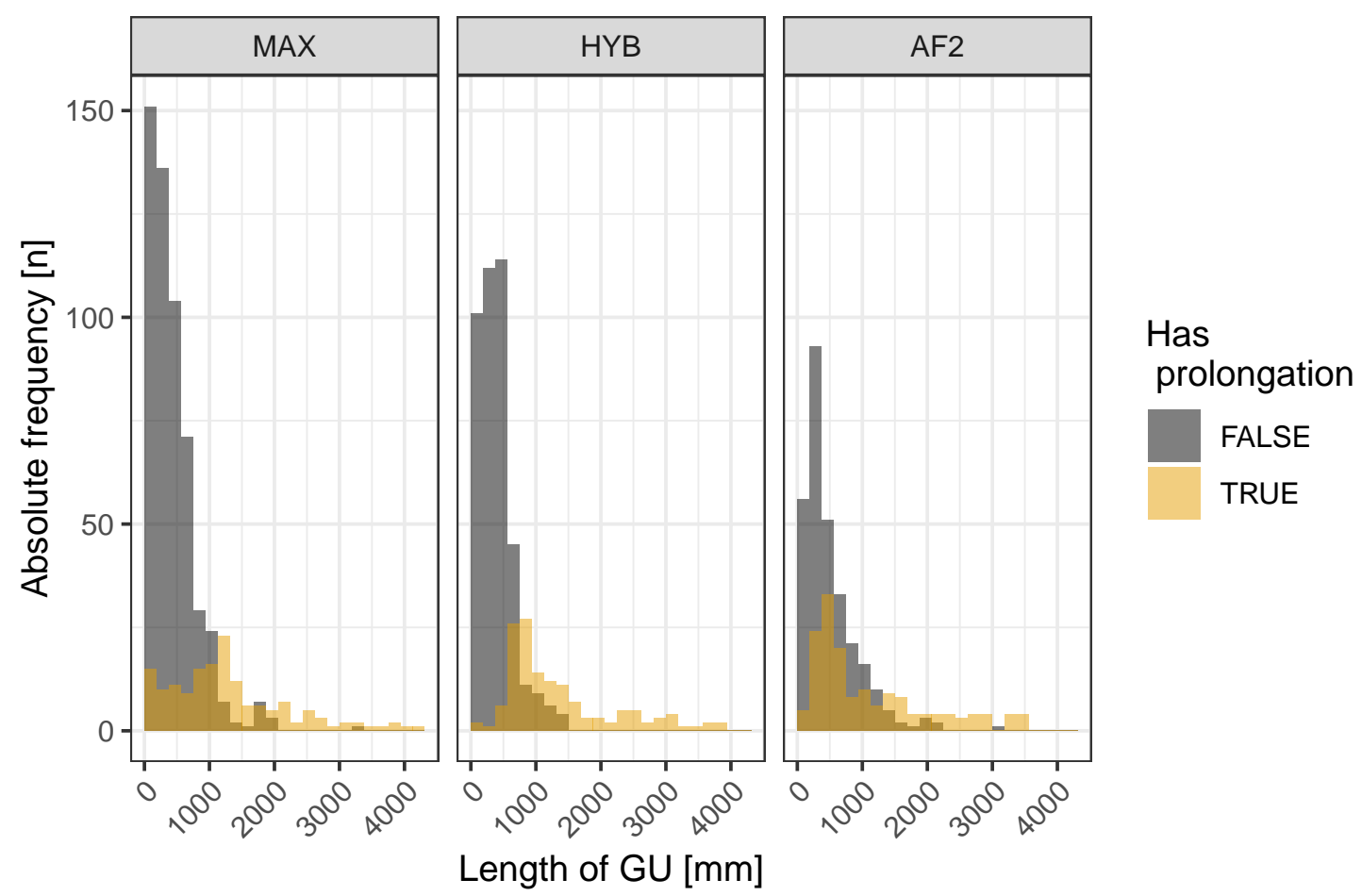

Figure 3.16: Distribution of count of GU by their length separated by clone and whether they have a prolongation child-GU or not. Considered here are only GUs that are not part of branch order 0 on the main stem and that can possibly have a prolongation (age $>1)$.

The first general observation is that the absolute frequency of short GUs is higher than that of long GUs for all clones. Also the number of GUs with no prolongation (gray bars) is considerably higher than that of GUs with a prolongation. The proportion between those two cases changes however with increasing length. There are small differences by clone. Nonetheless, beyond a length of $2000 \mathrm{~mm}$ almost all GUs have developed prolongation shoots. A generalized linear model was fit with the binary distributed response has $P L$ coding a present or no prolongation as 1 respectively 0 . Variables like order and age were tested for their suitability as covariates. A comparably simple model including the variables clone, GU length $l$ and their interaction was chosen by variable selection. The model diagnostics are given in Table 3.8 . 
Table 3.8: Summary of generalized linear model fit for estimating $\eta_{\text {has } P L}$ of the probability $p_{\text {has } P L}$ of growth units having a prolongation growth unit. Independent variables are length of growth unit $l$ and the clonal affiliation clone. The colon sign stands for the interaction of two variables. The parameterized model function is given in Equation 3.6.

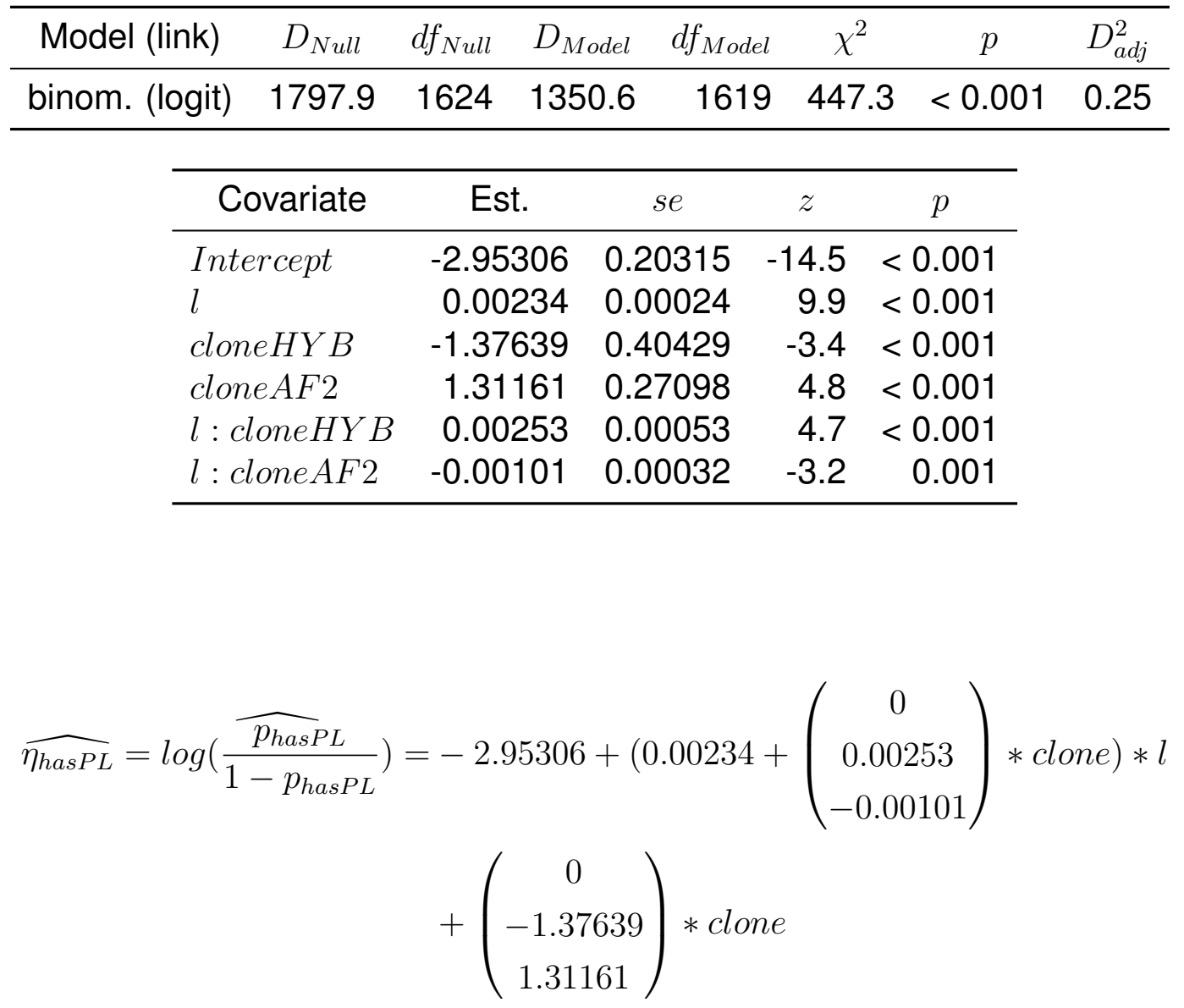

Analyzing the model's coefficients, the probability of a prolongation to grow increases through the link function with the GU's length. The rate of increase is changed with clonal affiliation, signified by the interaction terms. The increase with length is significantly higher for 'Hybride 275' in comparison to 'Max 1'. This relationship is reversed for 'AF2' and 'Max 1'. For the general clonal factor, this sequence is again reversed. These findings can be acknowledged in Figure 3.16 , 'AF2' has a higher probability for prolongation GUs on shorter shoots but the probability does not increase much in magnitude. For 'Hybride 275 ' the probability of prolongation GUs is low below a length of $500 \mathrm{~mm}$ for the mother-GU. The 
probability then increases to a greater extent than for 'AF2'. The probabilities for 'Max 1' are settled on a medium level between the 2 other clones. While the omnibus test points towards a model with explanatory value, the overall predictive power is rather low with $D_{a d j}^{2}=0.25$.

For modeling the probability of lateral buds to develop a regular daughter $\mathrm{GU}$, a generalized linear model was parameterized again with the binary response hasLat which implies, per node, a regular, lateral shoot present as 1 and none as 0 . The model that was chosen in this case relies on the length $l$ of the GU that the node belongs to, the relative position relPos of the node on that GU, the age (in the form of year $t$ that the shoot developed in), whether the GU was part of the main stem main as well as clone. The relationship of the response has Lat and the GU's length is shown in Figure 3.17. In this subsample, the overall count of nodes on short-length GUs is higher and decreases with increasing length. This observation is amplified by the fact that the node count would increase more sharply for an increasing number of longer GUs since they also imply a higher node count. The overall number of nodes with no lateral shoots is again much higher than that of those with lateral shoots. The occurrence of the latter in relation to nodes with no lateral children becomes more and more considerable as a proportion above $1000 \mathrm{~mm}$ GU length. 


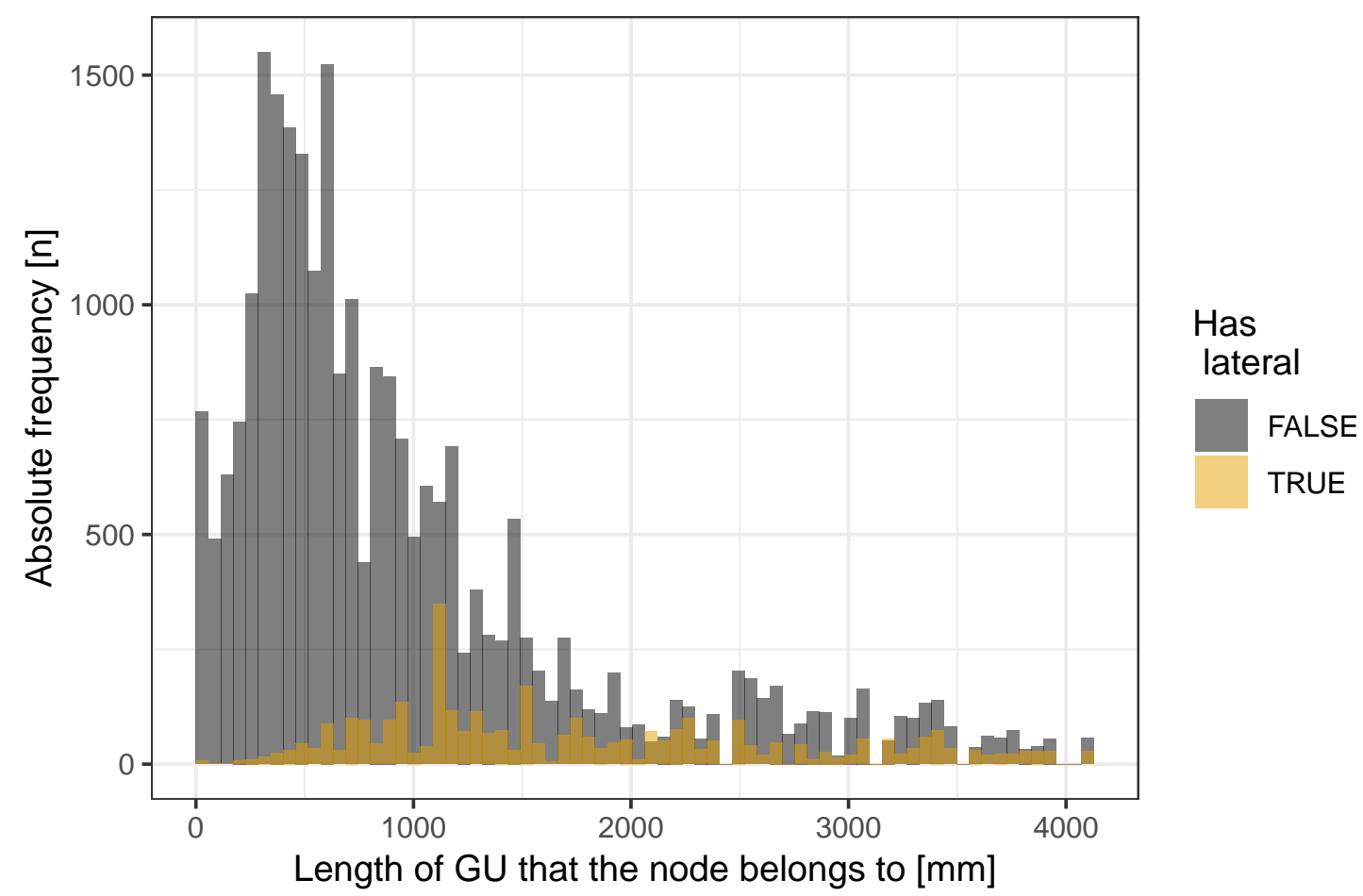

Figure 3.17: Distribution of count of nodes by length of the whole GU separated by whether the nodes have a regular, lateral child GU or not. Considered here are only nodes that can possibly have a regular lateral shoot $($ age $>1)$.

Looking at the distribution of the two states of the response within a GU (see Figure 3.18), a higher frequency of nodes at the lower and upper relative positions close to 0 and $100 \%$ can be observed. This is based on the property that internodes close to the GU base and tip are shorter than towards the middle which is further amplified by estimating relative positions of "empty" nodes for dtd data (see section 3.1.3). This will be scrutinized later on (see Figure 3.26). The probability of lateral shoots occurring increases towards the GU's tip. 


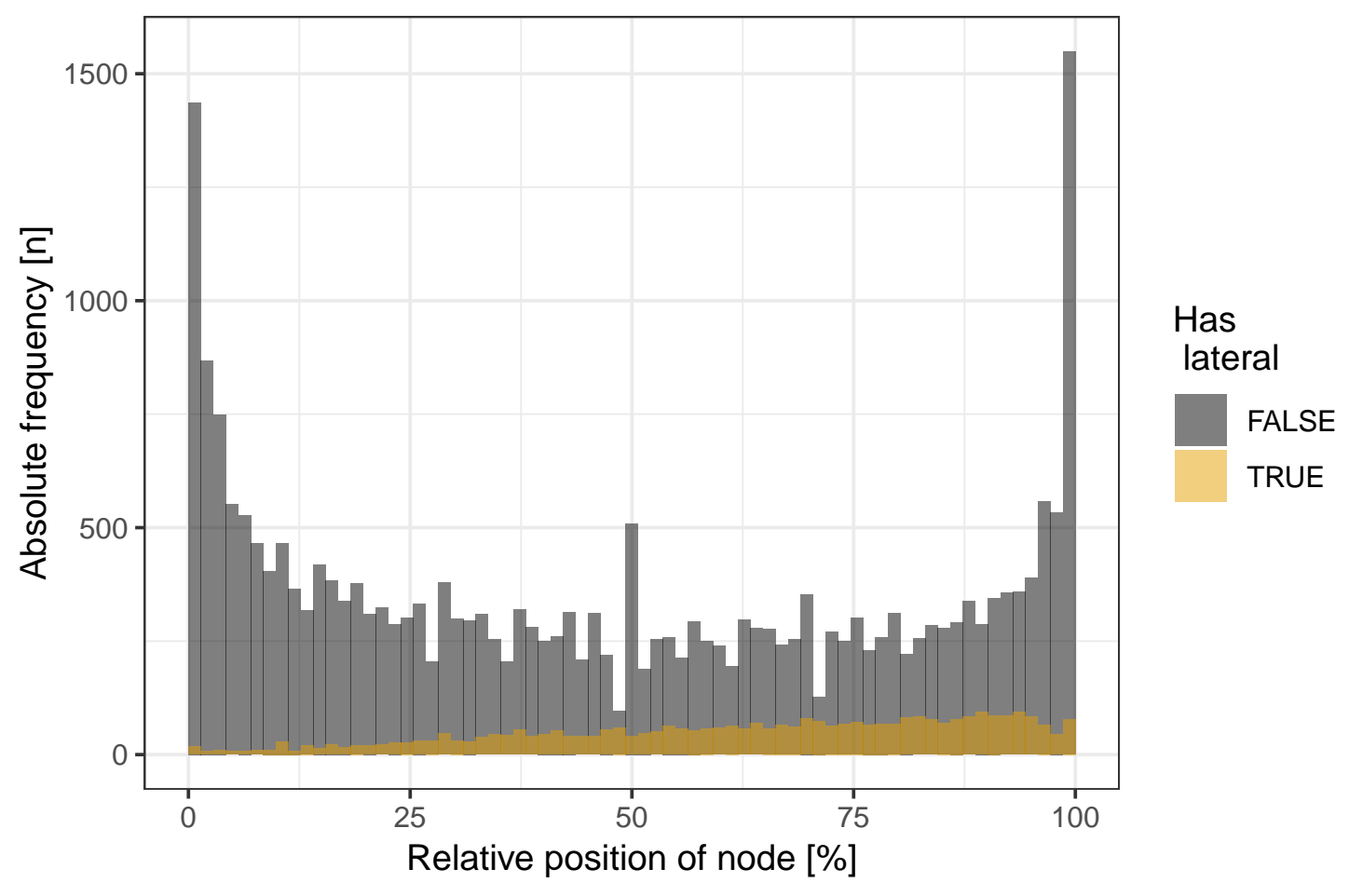

Figure 3.18: Distribution of count of nodes by their relative position on the GU separated by whether the nodes have a regular, lateral child GU or not. Considered here are only nodes that can possibly have a regular lateral shoot (age $>1)$.

The summaries for the GLM that incorporates these findings are given in Table 3.9. 
Table 3.9: Summary of generalized linear model fit for estimating $\eta_{\text {hasLat }}$ of the probability $p_{\text {hasLat }}$ of lateral nodes having a child GU. Variable $l$ resembles the growth unit length, relPos the relative position of the node on the GU, $t$ the rotation year, main whether the GU belongs to the main axis or not and clone the clonal affiliation. The $p$ values are based on $F$ tests. The colon sign stands for the interaction of two variables. The parameterized model function is given in Equation 3.6.

\begin{tabular}{|c|c|c|c|c|c|c|c|}
\hline Model (link) & $D_{\text {Null }}$ & $d f_{\text {Null }}$ & $D_{M o}$ & & $d f_{\text {Model }}$ & $F$ & $p$ \\
\hline q.-binom. (logit) & $0.84 \quad 21284.6$ & 28825 & 1648 & & 28811 & 407.6 & $<0.001$ \\
\hline \multicolumn{2}{|c|}{ Covariate } & \multicolumn{2}{|c|}{ Est. } & & se & $t$ & $p$ \\
\hline \multicolumn{2}{|c|}{ Intercept } & \multicolumn{2}{|c|}{-2.77332} & \multicolumn{2}{|c|}{0.25165} & -11.0 & $<0.001$ \\
\hline \multirow{2}{*}{\multicolumn{2}{|c|}{ relpos }} & \multicolumn{2}{|c|}{-0.00093} & \multicolumn{2}{|c|}{0.00010} & -9.1 & $<0.001$ \\
\hline & & \multicolumn{2}{|c|}{0.50601} & \multicolumn{2}{|c|}{0.14198} & 3.6 & $<0.001$ \\
\hline \multicolumn{2}{|l|}{ relPos } & \multicolumn{2}{|c|}{-0.77608} & \multicolumn{2}{|c|}{0.12765} & -6.1 & $<0.001$ \\
\hline \multicolumn{2}{|c|}{$\operatorname{main} T R U E$} & \multicolumn{2}{|c|}{1.37742} & \multicolumn{2}{|c|}{0.11721} & 11.8 & $<0.001$ \\
\hline \multicolumn{2}{|c|}{ clone $H Y B$} & \multicolumn{2}{|c|}{-0.93347} & \multicolumn{2}{|c|}{0.17423} & -5.4 & $<0.001$ \\
\hline \multicolumn{2}{|c|}{ clone $A F 2$} & \multicolumn{2}{|c|}{-1.48688} & \multicolumn{2}{|c|}{0.20014} & -7.4 & $<0.001$ \\
\hline \multicolumn{2}{|c|}{$l:$ relPos } & \multicolumn{2}{|c|}{0.00145} & \multicolumn{2}{|c|}{0.00007} & 21.6 & $<0.001$ \\
\hline \multicolumn{2}{|l|}{$l: t$} & \multicolumn{2}{|c|}{0.00098} & \multicolumn{2}{|c|}{0.00005} & 18.7 & $<0.001$ \\
\hline \multicolumn{2}{|c|}{$l: \operatorname{mainT} R U E$} & \multicolumn{2}{|c|}{-0.00039} & \multicolumn{2}{|c|}{0.00004} & -9.1 & $<0.001$ \\
\hline \multicolumn{2}{|c|}{ relPos: mainTRUE } & \multicolumn{2}{|c|}{-1.16957} & 0.13 & 3230 & -8.8 & $<0.001$ \\
\hline$t$ : clone & $H Y B$ & 0.09 & 145 & 0.11 & 1625 & 0.8 & 0.432 \\
\hline$t$ : clone & $A F 2$ & 0.61 & 136 & & 2007 & 5.1 & $<0.001$ \\
\hline $\operatorname{mainTl}$ & $U E$ : clone $H Y B$ & 0.80 & 766 & & 1366 & 7.1 & $<0.001$ \\
\hline $\operatorname{mainTl}$ & $U E$ : clone $A F 2$ & 0.58 & 255 & 0.10 & 0646 & 5.5 & $<0.001$ \\
\hline
\end{tabular}




$$
\begin{aligned}
\widehat{\eta_{\text {hasLat }}}=\log \left(\frac{\widehat{p_{\text {hasLat }}}}{1-p_{\text {hasLat }}}\right)= & -2.77332+(-0.00093-0.00039 * \text { main }) * l \\
& +(0.50601-1.16957 * \text { main }) * \text { relPos } \\
& +\left(-0.77608+\left(\begin{array}{c}
0 \\
0.09145 \\
0.61136
\end{array}\right) * \text { clone }\right) * t \\
& +\left(1.37742+\left(\begin{array}{c}
0 \\
0.80766 \\
0.58255
\end{array}\right) * \text { clone }\right) * \text { main } \\
& +0.00145 * l * \text { relPos }+0.00098 * l * t \\
& +\left(\begin{array}{c}
0 \\
-0.93347 \\
-1.48688
\end{array}\right) * \text { clone }
\end{aligned}
$$

The dispersion parameter $\Phi=0.84$ points towards a slight underdispersion, the chosen model family is quasi-binomial. While the null-hypothesis for the omnibus $F$-test could be rejected as before, the $D_{a d j}^{2}$ of 0.23 is again rather low but could not be further improved. Looking at the coefficients, it needs consideration that the relative position, opposed to Figure 3.18, must be entered here as a fraction and not in \%-values. It is also of interest that the variables $t$ and $l$ have negative coefficients although their influence was established as positive. In this case the interactions of these metric variables with either themselves or relPos are positive and counteract this effect by having more impact in estimating $\eta$. So GUs that are longer and younger have a higher probability for lateral shoots to develop. This probability further increases with a more distal node position on the shoot.

The differences between clones seem small. Considering the main clonal effect, 'Max 1' has a higher probability to grow lateral shoots than 'Hybride 275' and 'AF2'. This is reversed within the coefficient for the interaction of clone and $t$ meaning that with younger growth units these clones have a higher probability for developing lateral shoots. Furthermore, 'AF2' and 'Hybride 275' have a positive interaction with the factor main, which decreases the differences between 
clones on the main stem. The effect for main implies a higher probability for lateral shoots to develop on main stems of all clones. This is however compensated to some degree by the negative interaction effects of main with $l$ and relPos.

\subsubsection{Count of Internodes per Growth Unit}

Based on visual assessment, variables like age, branch order and being part of the main stem or not determine the length and the count of internodes per growth unit. The approach to estimate this as a response was divided into 4 cases:

- GUs developing in the first rotation year.

- Prolongation GUs developing after the first rotation year.

- Lateral, regular GUs developing after the first rotation year.

- Lateral, sylleptic GUs which may develop during all 3 rotation years.

For the first case, possibly influential variables like number of stems per stool, clone and whether the growth unit was initiating a main or minor stem were considered. Only the latter variable exhibited a significant effect which is exemplified in Figure 3.19, It should be emphasized here again that this variable is based on systematic assignment and choice. Also, the sample sizes and variances differ substantially.

Model diagnostics for the negative binomial GLM that was fit to this data are given in Table 3.10. With 0.18 , the $D_{a d j}^{2}$ is again rather low although the null deviance does not seem too large and comparing the residual deviance to the residual degrees of freedom is satisfactory which is complemented by the results of the comparison to the null model. 


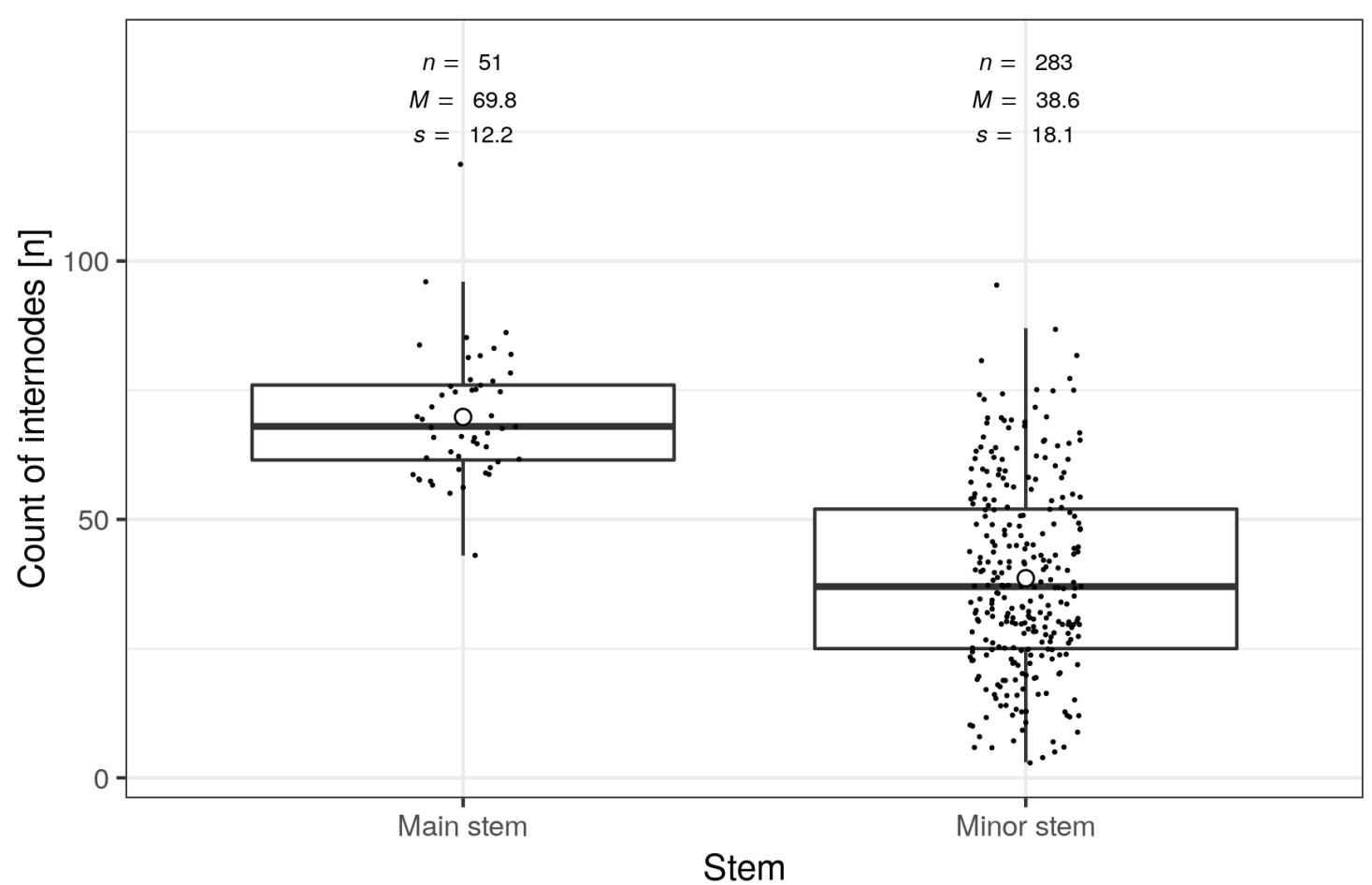

Figure 3.19: Count of internodes per GU by affiliation to a main or minor stem. Included are only GUs that have elongated in the first vegetation period after cutting (age $=3, t=1)$

Table 3.10: Summary of generalized linear model fit for estimating $\eta$ of the response innInit of count of internodes for GUs from the first year of the rotation. Independent variable main indicates if a GU belongs to a main or minor stem.

\begin{tabular}{|c|c|c|c|c|c|c|}
\hline Model (link) & $\hat{\theta}(s e)$ & $D_{\text {Null }} \quad d$ & $d f_{\text {Null }}$ & $D_{\text {Model }}$ & $d f_{\text {Model }}$ & $L R T$ \\
\hline neg. bin. $(\log )$ & $5.27(0.47)$ & 435.2 & 333 & 352.9 & 332 & $73.2<0$ \\
\hline & Covariate & Est. & & se & $z$ & $p$ \\
\hline & $\begin{array}{l}\text { Intercept } \\
\text { mainTRUE }\end{array}$ & $\begin{array}{l}3.65391 \\
0.59206\end{array}$ & & $\begin{array}{l}.02760 \\
.06900\end{array}$ & $\begin{array}{r}132.4 \\
8.6\end{array}$ & $\begin{array}{l}<0.001 \\
<0.001\end{array}$ \\
\hline
\end{tabular}


Modeling the number of internodes for prolongation growth units depends on more explanatory variables. The selection procedure identified the length of the mother GU lMother, the branch order order (which is the same for mother and prolongation), the main/minor stem assignment main as well as clonal affiliation clone as essential. The relationship between length of mother $\mathrm{GU}$ and the number of internodes of the prolongation GU can be assessed in Figure 3.20. The correlation is positive which can be confirmed from the model coefficients in Table 3.10. While this implies longer prolongation GUs for longer mother GUs, there is still a decrease to some degree since the increase does not resemble a 1:1 relation. Regarding the clonal effect, 'AF2' produces longer prolongation GUs in opposition to 'Hybride 275' and 'Max 1' which do not differ significantly.

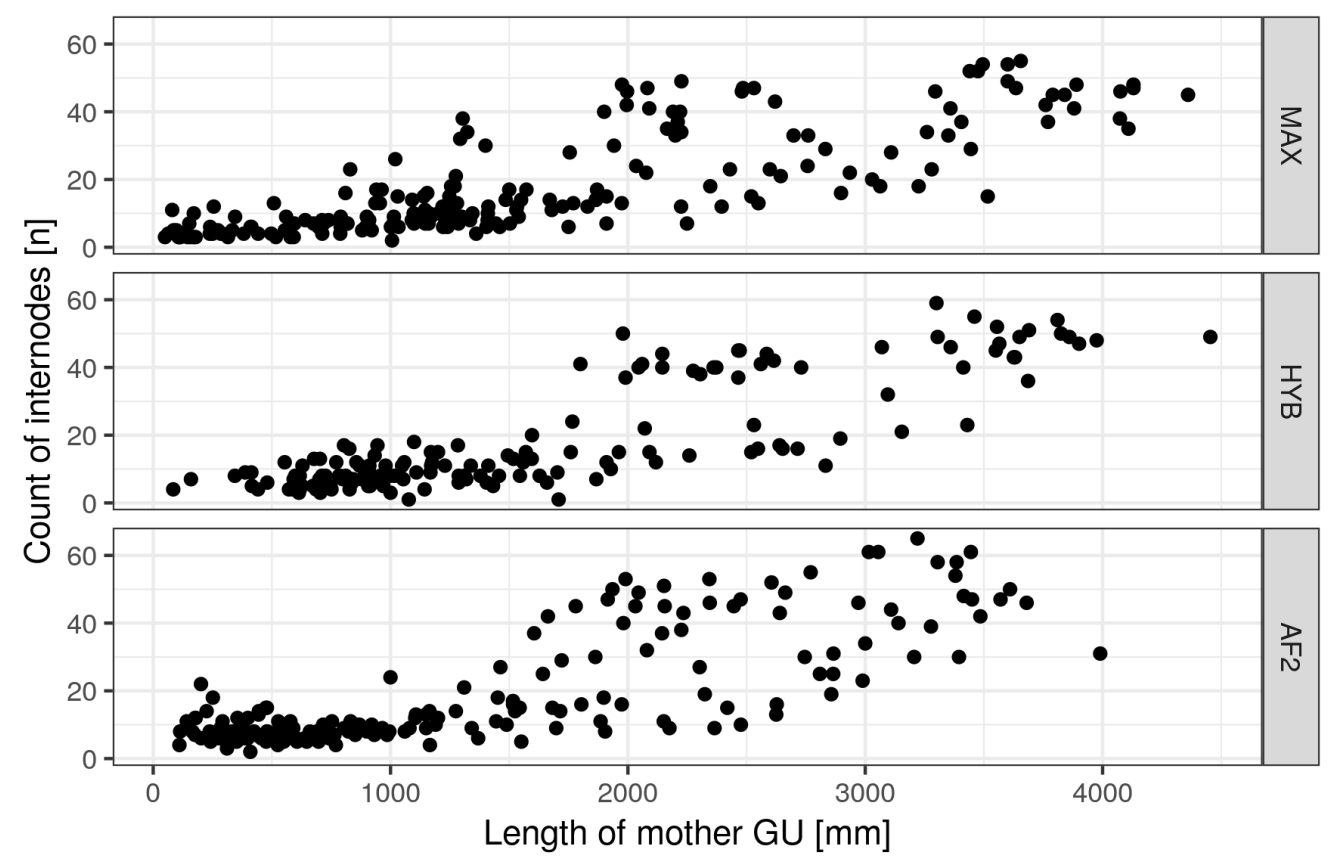

Figure 3.20: Count of internodes per prolongation GU by the length of its mother GU.

To pervade the influence of main and order, Figure 3.21 shows the distribution of internode count within the levels of these variables crossed. 

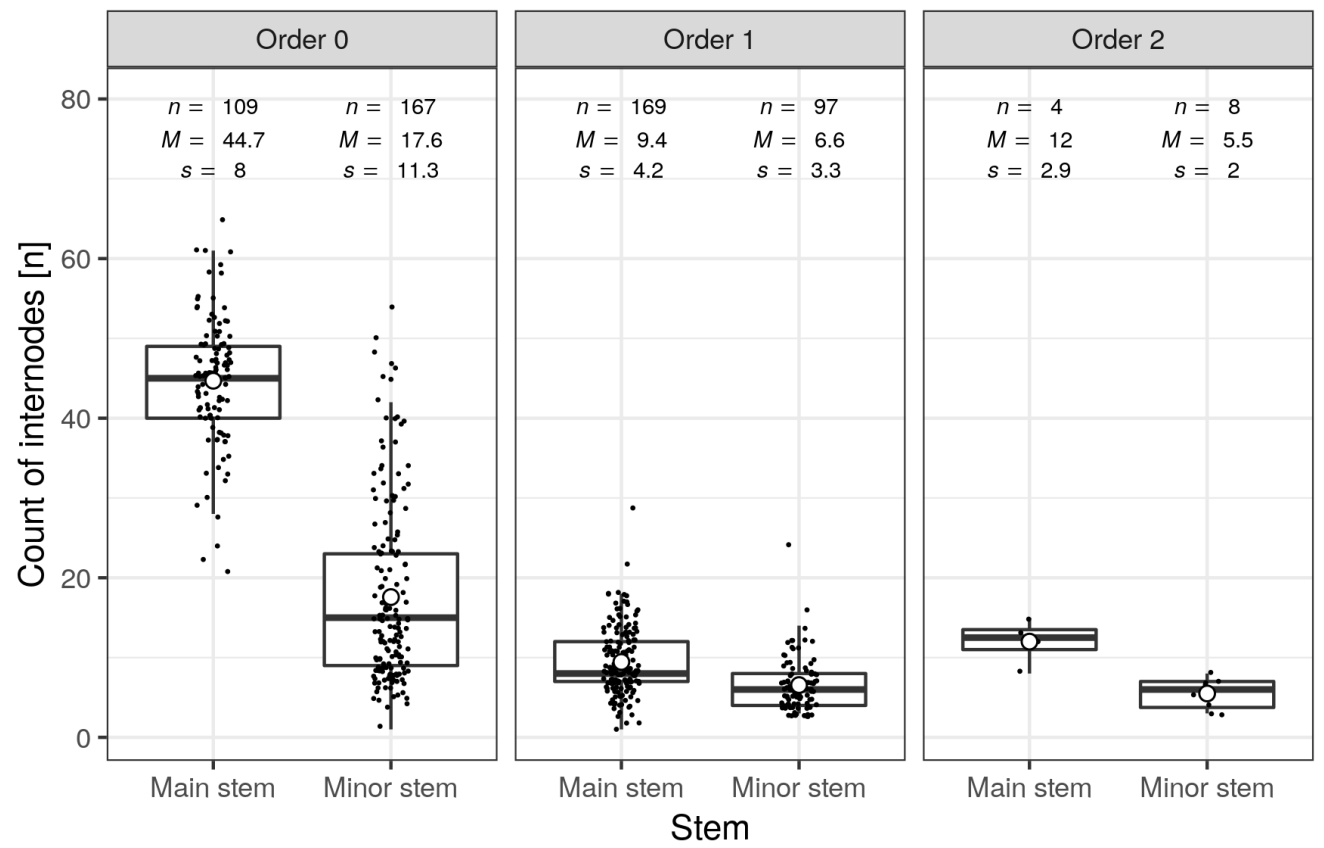

Figure 3.21: Count of internodes per prolongation GU by its branch order (in facets) and whether they belong to a main or minor stem.

With increasing branch order, the number of internodes decreases sharply from branch order 0 to the first order and then stays on a comparable level for the second branch order. Overall, main stems tend to have more internodes in prolongation GUs than it's the case for minor stems. This difference is reduced however in the first and second branch order. This can be confirmed by the interaction effect (see Table 3.11). Again, the mechanism for determining main and minor stems as well as the differing group sizes must be taken into consideration here. Especially for the second branch order any statements have to be interpreted carefully due to the very low amount of prolongation units in this category. The $D_{\text {adj }}^{2}=0.80$ points towards a high predictive power (see Table 3.11). The negative binomial model family was used for GLM fitting to accommodate for the overdispersion. 
Table 3.11: Summary of generalized linear model fit for estimating $\eta_{i n n P L}$ of the response innPL of count of internodes for prolongation GUs. The chosen independent variable are main, indicating if a GU belongs to a main or minor stem, the length of the mother GU (lMother), the branch order (order) and clonal affiliation (clone). The colon sign stands for the interaction of two variables. The parameterized model function is given in Equation 3.7.

\begin{tabular}{|c|c|c|c|c|c|}
\hline Model (link) & $\hat{\theta}(s e)$ & $d f_{\text {Null }}$ & $D_{\text {Model }} \quad d f$ & $f_{\text {Model }}$ & $L R T$ \\
\hline neg. bin. (log) & $13.73(1.59) \quad 281$ & 553 & 550.4 & 545 & $880.1<0.00$ \\
\hline & Covariate & Est. & se & $z$ & $p$ \\
\hline Inte & rcept & 2.10834 & 0.06045 & 34.9 & $9<0.001$ \\
\hline$l M c$ & ther & 0.00034 & 0.00002 & 14.8 & $3<0.001$ \\
\hline ordt & & -0.47966 & 0.06530 & -7.3 & $3<0.001$ \\
\hline orde & & -0.60404 & 0.18609 & -3.2 & 0.001 \\
\hline mai & $\imath T R U E$ & 0.70071 & 0.04506 & 15.6 & $6<0.001$ \\
\hline clon & $H Y B$ & -0.05152 & 0.04066 & -1.3 & 0.205 \\
\hline clon & $A F 2$ & 0.16158 & 0.03906 & 4.1 & $1<0.001$ \\
\hline orde & $r 1: \operatorname{main} T R U E$ & -0.44613 & 0.07231 & -6.2 & $2<0.001$ \\
\hline ordt & $r 2: \operatorname{mainTRUE}$ & -0.15798 & 0.27072 & -0.6 & 0.56 \\
\hline
\end{tabular}

$$
\begin{aligned}
\widehat{\eta_{\text {innPL }}}=\log \widehat{\left(\mu_{\text {inn } P L}\right)}= & 2.10834+0.00034 * \text { lMother }+\left(\begin{array}{c}
0 \\
-0.47966 \\
-0.60404
\end{array}\right) * \text { order } \\
& +0.70071 * \text { main }+\left(\begin{array}{c}
0 \\
-0.05152 \\
0.16158
\end{array}\right) * \text { clone } \\
& +\left(\begin{array}{c}
0 \\
-0.44613 \\
-0.15798
\end{array}\right) * \text { order } * \text { main }
\end{aligned}
$$

For estimating the length of regular, lateral GUs, first, two cases were distinguished: development of a short shoot versus development of a long shoot. The occurrence of the first case became apparent during the measurement of the leaf 
morphology where several leaf-bearing shoots with a length below $10 \mathrm{~mm}$ were observed. This will be elaborated in more detail in the section for leaf morphology (3.2.9). The threshold of $10 \mathrm{~mm}$ was deliberately chosen and seems arbitrary since such separations seldom resemble the rather gradual transitions in shoot morphology. For modeling the structure however, it seemed more appropriate to accommodate for the excess number of shoots in this length range. Therefore a binomial GLM was fitted to implement a random number generator to distinguish between the two cases. During variable selection it became evident that the two variables relative position on mother-GU relPos and assignment to main or minor stem main were most influential towards the response. In Figure 3.22 the influence of the relative position is demonstrated. As already asserted, the general number of regular, lateral shoots increases with an increasingly distal relative position. Distinguishing between short and long shoots, as described here, shows that the former have a higher probability of occurring in middle to lower part of a GU while the latter develop more often towards the shoot tip.

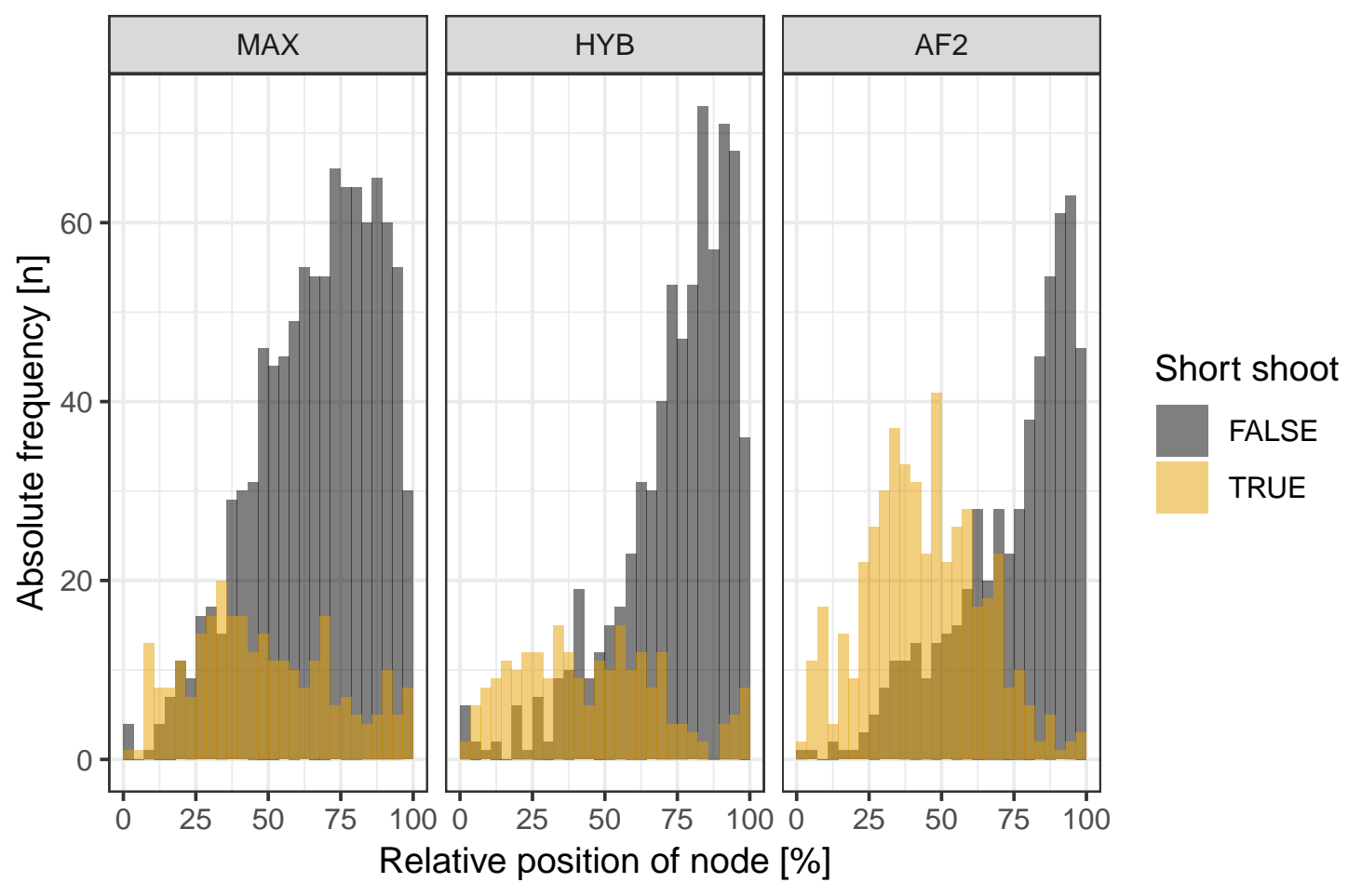

Figure 3.22: Distribution of long versus short shoots by their relative position on the mother GU separated by clone. 
The influence of the second important variable main can be assessed with Table 3.12. As can be verified from Figure 3.22, clone 'AF2' has an overall higher probability for developing short shoots and also the differences between main and minor stems is much more pronounced.

Table 3.12: Frequency table for total number of regular, lateral shoots grouped by clone and main/minor stem. The total amount is further divided into short shoots $(l<10 \mathrm{~mm})$ and long shoots $(l>=10 \mathrm{~mm})$. Included are only shoots with their length data measured.

\begin{tabular}{llrrr}
\hline Clone & Stem & Total lateral [n] & Short shoots [n] & Long shoots [n] \\
\hline MAX & minor & 462 & 44 & 418 \\
MAX & main & 796 & 230 & 566 \\
HYB & minor & 309 & 11 & 298 \\
HYB & main & 620 & 218 & 402 \\
AF2 & minor & 255 & 1 & 254 \\
AF2 & main & 777 & 470 & 307 \\
\hline
\end{tabular}

The summary for the model that was parameterized to reflect these relations is given in Table 3.13. With the given set of selected variables a $D_{a d j}^{2}=0.51$ was achieved which points towards an intermediate predictive power. No relevant over- or underdispersion was detected which is why the binomial model family was utilized. 
Table 3.13: Summary of generalized linear model fit for estimating $\eta_{s h r t S h}$ of the probability $p_{\text {shrtSh }}$ of lateral growth units being a short shoot with $l$ $<10 \mathrm{~mm}$ instead of a long shoot with $l>=10 \mathrm{~mm}$. Variable $l$ Mother resembles the mother growth unit's length, relPos the relative position of the node on that GU, order the branch order of the to be developed shoot (ranging from order 1 to 3 in this case), main the assignment to main/minor stems and clone the clonal affiliation. The colon sign stands for the interaction of two variables. The parameterized model function is given in Equation 3.8.

\begin{tabular}{|c|c|c|c|c|c|c|}
\hline Model (link) & $d f_{\text {Null }}$ & $D_{\text {Model }}$ & $d f_{\text {Model }}$ & & $p$ & $D_{a d j}^{2}$ \\
\hline binom. (logit) & $3946.7 \quad 3218$ & 1919.3 & $3207 \quad 2027$ & & $<0.001$ & 0.51 \\
\hline \multicolumn{2}{|c|}{ Covariate } & Est. & se & $z$ & \multicolumn{2}{|l|}{$p$} \\
\hline \multicolumn{2}{|l|}{ Intercept } & -0.96571 & 0.43685 & -2.2 & \multicolumn{2}{|c|}{0.027} \\
\hline \multirow{2}{*}{\multicolumn{2}{|c|}{ lMother }} & -0.00081 & 0.00016 & -5.1 & \multicolumn{2}{|c|}{$<0.001$} \\
\hline & & -3.44351 & 0.38883 & -8.9 & \multicolumn{2}{|c|}{$<0.001$} \\
\hline \multicolumn{2}{|l|}{ order 2} & 2.61210 & 0.29276 & 8.9 & \multicolumn{2}{|c|}{$<0.001$} \\
\hline \multicolumn{2}{|l|}{ order 3} & -8.03203 & 349.51302 & -0.0 & \multicolumn{2}{|c|}{0.982} \\
\hline \multicolumn{2}{|c|}{$\operatorname{mainT} R U E$} & 1.04001 & 0.24693 & 4.2 & \multicolumn{2}{|c|}{$<0.001$} \\
\hline \multicolumn{2}{|c|}{ clone $H Y B$} & 1.11721 & 0.53340 & 2.1 & 0.036 & \\
\hline \multicolumn{2}{|c|}{ cloneAF2 } & -1.27371 & 1.09771 & -1.2 & 0.246 & \\
\hline \multirow{2}{*}{\multicolumn{2}{|c|}{$\begin{array}{l}\text { relPos : clone } H Y B \\
\text { relPos : clone } A F 2\end{array}$}} & -2.04178 & 0.64220 & -3.2 & \multicolumn{2}{|c|}{0.001} \\
\hline & & -2.78233 & 0.66340 & -4.2 & \multicolumn{2}{|c|}{$<0.001$} \\
\hline \multicolumn{2}{|c|}{ mainTRUE : clone $H Y B$} & 0.66637 & 0.45049 & 1.5 & \multirow{2}{*}{$\begin{array}{r}0.139 \\
<0.001\end{array}$} & \\
\hline \multicolumn{2}{|c|}{$\operatorname{main} T R U E:$ clone $A F 2$} & 4.25351 & 1.04172 & 4.1 & & \\
\hline
\end{tabular}




$$
\begin{aligned}
& \widehat{\eta_{\text {shrtSh }}}=\log \left(\frac{\widehat{p_{\text {shrt } S h}}}{1-p_{\text {shrtSh }}}\right)=-0.96571-0.00081 * \text { lMother } \\
& +\left(-3.44351+\left(\begin{array}{c}
0 \\
-2.04178 \\
-2.78233
\end{array}\right) * \text { clone }\right) * \text { relPos } \\
& +\left(\begin{array}{c}
0 \\
2.61210 \\
-8.03203
\end{array}\right) * \text { order } \\
& +\left(1.04001+\left(\begin{array}{c}
0 \\
0.66637 \\
4.25351
\end{array}\right) * \text { clone }\right) * \text { main } \\
& +\left(\begin{array}{c}
0 \\
1.11721 \\
-1.27371
\end{array}\right) * \text { clone }
\end{aligned}
$$

From the model coefficients it can be deducted that the length of the mother GU has a negative effect on the probability of a short shoot occurring. This is further enhanced by the effect of branch order which points towards a higher probability in the second branch order. The coefficient for the third order is negative but not significant, due to the high standard error which, again, is caused by the small number of observations in this group. The overall tendency seems to be that short shoots (of the second branch order) occur more often on the shorter growth units of the first branch order. The effect of relative position relPos being negative and that of main being positive with the large interaction effect of main : clone for 'AF2' supports the observations made in Figure 3.22 and Table 3.12.

After partitioning whether a short shoot or a long shoot will be developed, the number of internodes can be estimated accordingly. This necessitates two separate models. The summary for estimating the number of internodes $i N o d e N$ of short shoots is organized in Table 3.14. The parameter $\Phi$ was estimated with 0.25 by the quasi-Poisson procedure, indicating an underdispersion. Selecting the independent variables like length of mother GU, main or minor stem and clone resulted in a high goodness of fit of $D_{a d j}^{2}=0.73$. 
Table 3.14: Summary of generalized linear model fit for estimating $\eta_{i N o d e N}$ of the response $i$ Node $N$ meaning number of internodes for short shoots with $l<10 \mathrm{~mm}$. Variable $l$ Mother resembles the mother growth unit's length, main the assignment to main/minor stems and clone the clonal affiliation. The colon sign stands for the interaction of two variables. The parameterized model function is given in Equation 3.9

\begin{tabular}{|c|c|c|c|c|c|c|}
\hline Model (link) & $\Phi$ & $D_{\text {Null }} \quad d f$ & $d f_{\text {Null }} \quad D_{\text {Model }}$ & $d f_{\text {Model }}$ & $F$ & $p$ \\
\hline q.-poiss. (log) & 0.25 & 973.5 & 257.5 & 916 & 355.1 & $<0.001$ \\
\hline \multicolumn{3}{|c|}{ Covariate } & Est. & se & $t$ & $p$ \\
\hline \multicolumn{3}{|c|}{ Intercept } & -0.51500 & 0.14884 & -3.5 & $<0.001$ \\
\hline \multicolumn{3}{|c|}{ lMother } & 0.00049 & 0.00006 & 7.6 & $<0.001$ \\
\hline \multicolumn{3}{|c|}{$\operatorname{mainT} R U E$} & 0.99209 & 0.13261 & 7.5 & $<0.001$ \\
\hline \multicolumn{3}{|c|}{ clone $H Y B$} & 0.43884 & 0.32649 & 1.3 & 0.179 \\
\hline \multicolumn{3}{|c|}{ clone $A F 2$} & 1.88081 & 0.30013 & 6.3 & $<0.001$ \\
\hline \multicolumn{3}{|c|}{ lMother : clone $H Y B$} & -0.00036 & 0.00007 & -4.8 & $<0.001$ \\
\hline \multicolumn{3}{|c|}{ lMother : cloneAF2 } & -0.00047 & 0.00010 & -4.8 & $<0.001$ \\
\hline \multicolumn{3}{|c|}{ mainTRUE : clone $H Y B$} & $\begin{array}{l}3 \\
3\end{array}$ & 0.31958 & 0.9 & 0.366 \\
\hline \multicolumn{3}{|c|}{ mainTRUE : clone AF2 } & -2.37426 & 0.28492 & -8.3 & $<0.001$ \\
\hline
\end{tabular}

$\begin{aligned} \widehat{\eta_{\text {iNodeN }}=\log \widehat{\left(\mu_{\text {iNodeN }}\right)}=} & -0.51500+\left(0.00049+\left(\begin{array}{c}0 \\ -0.00036 \\ -0.00047\end{array}\right) * \text { clone }\right) * \text { lMother } \\ & +\left(0.99209 *\left(\begin{array}{c}0 \\ 0.28901 \\ -2.37426\end{array}\right) * \text { clone }\right) * \text { main } \\ & +\left(\begin{array}{c}0 \\ 0.43884 \\ 1.88081\end{array}\right) * \text { clone }\end{aligned}$

Cognizable by the positive coefficients, a longer mother growth unit and growth units on the main stem develop short shoots with more internodes. The overall influence of clone increases from 'Max 1' to 'Hybride 275' and 'AF2' although only 
the differences between 'Max 1' and 'AF2' are significant. This is counteracted by the negative clonal influence on the coefficient for length of mother GU lMother for 'Hybride 275' and 'Max 1'. Additionally, 'AF2' has a clear negative interaction with the main variable. This resembles the observation during leaf structure measurement that 'AF2' has more short shoots with only 1 internode (see Section 3.2.9).

Moving on to estimating the number of internodes for regular, lateral long shoots, the independent variables that were identified as influential are the length of the mother GU lMother, the relative position of the lateral shoot on that mother GU relPos, the branch order of the lateral GU order, whether it is part of the main or a minor stem main, clonal affiliation clone and the interaction of lMother with main and clone. The relative position was most important in explaining variance of the response followed by length of mother, branch order and main/minor stem distinction. In Figure 3.23 the relationship with relPos can be examined.

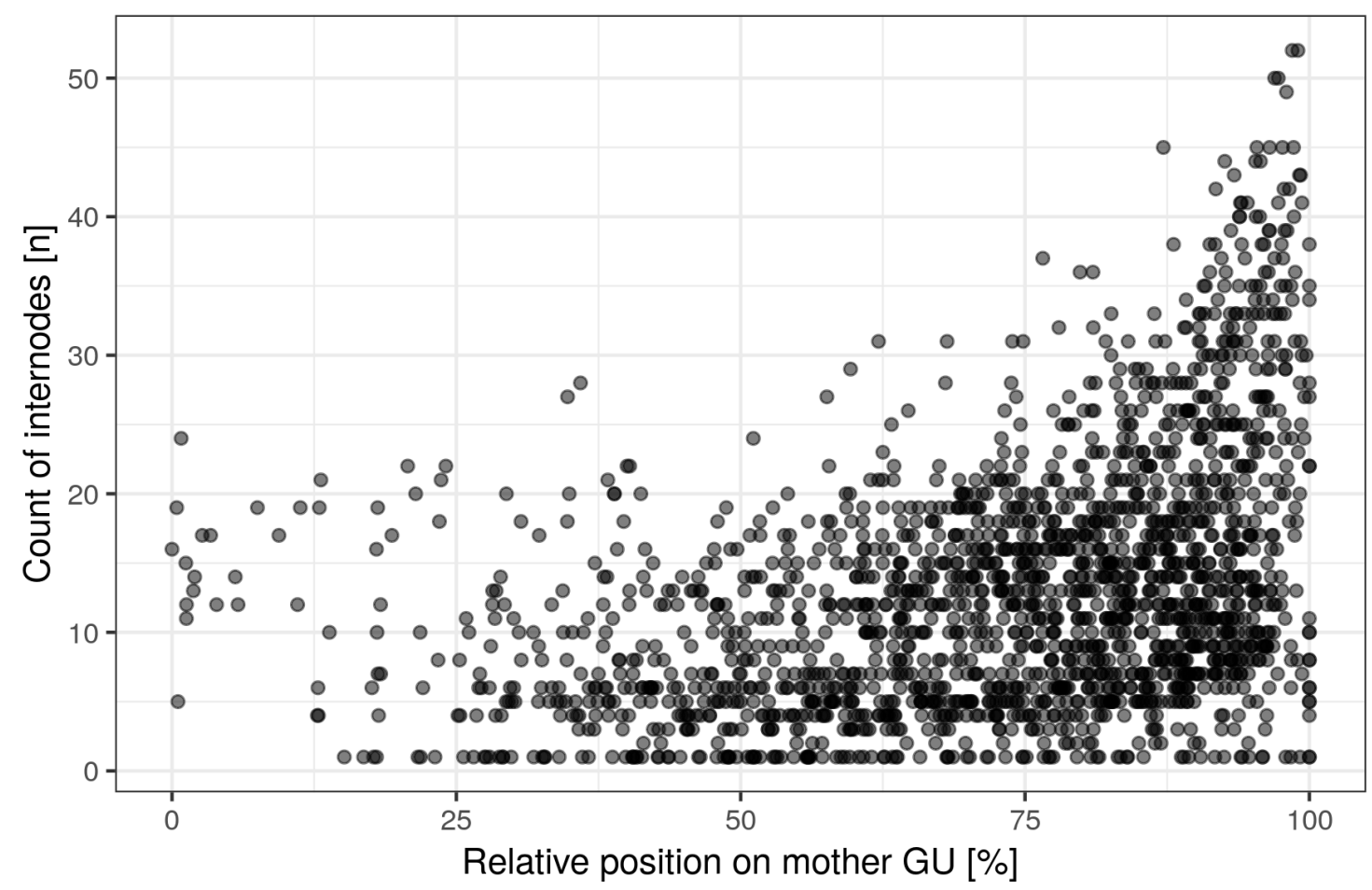

Figure 3.23: Count of internodes per regular, lateral GU by its relative position on the mother GU. Included are only long shoots with $l>=10 \mathrm{~mm}$.

The relationship between number of internodes for lateral shoots and length of 
mother GU is positive as can be seen in Figure 3.24. Lateral shoots on the main stem seem to develop higher internode counts than on minor stems (yellow versus black dots). The lateral shoot with the highest internode count was measured on a main stem of 'AF2'.

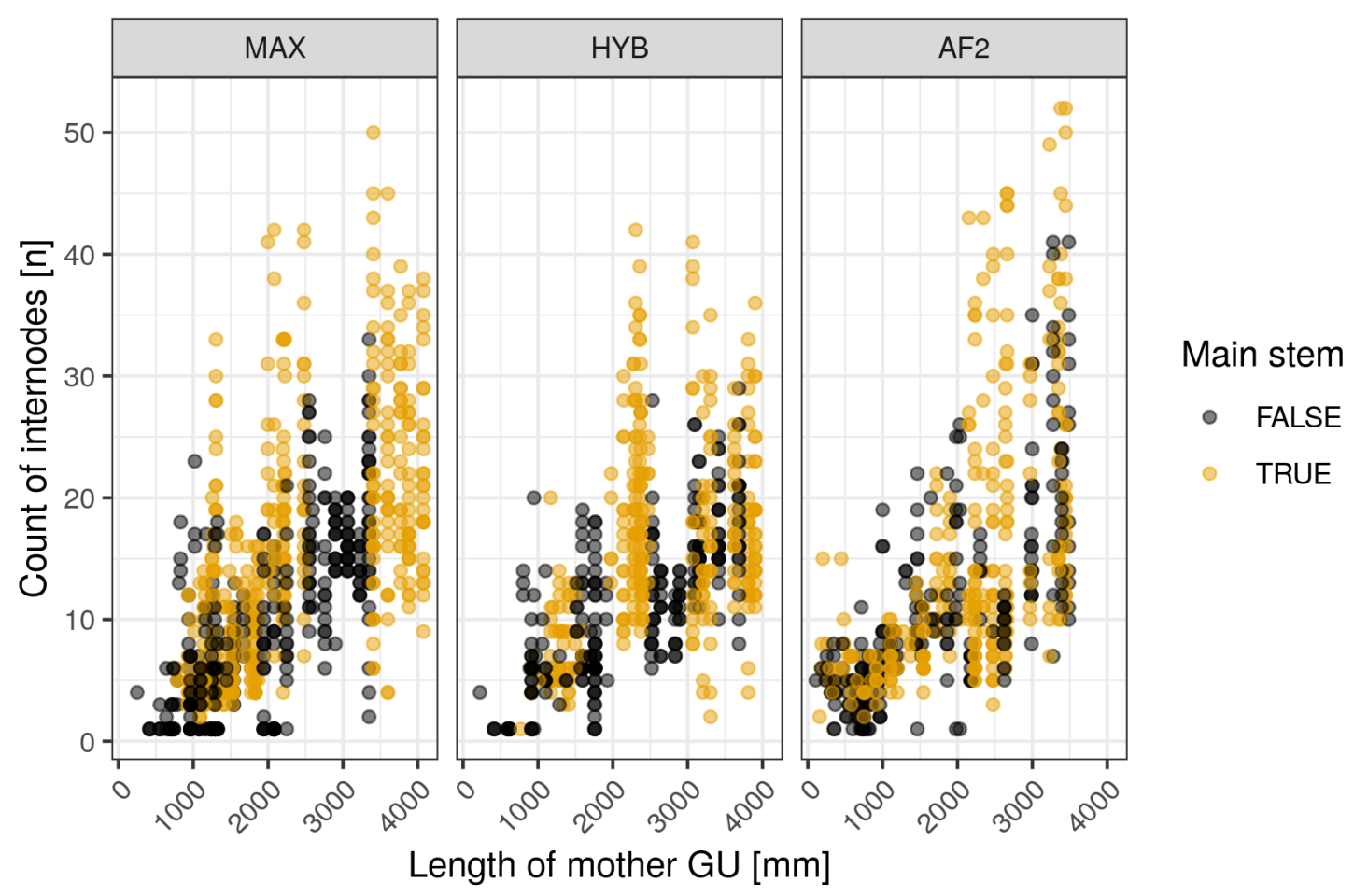

Figure 3.24: Count of internodes per regular, lateral GU by the length of its mother GU, separated by whether it belongs to a main stem or not and clone (the latter in facets). Included are only long shoots with $l>=10 \mathrm{~mm}$.

To quantify the direct and interaction influences of the given factors, the model summaries of the GLM that was parameterized for estimating the number of internodes of long shoots are included in Table 3.15. The negative binomial GLM performed better than the quasi-Poisson alternative, therefore the former was chosen. The predictive power indicated by $D_{a d j}^{2}=0.62$ is satisfactory. 
Table 3.15: Summary of generalized linear model fit for estimating $\eta_{i N o d e N}$ of the response $i$ Node $N$ meaning number of internodes for regular, lateral long shoots with $l>=10 \mathrm{~mm}$. Independent variables are the length of the mother GU (lMother), the relative position on the mother GU relPos, the branch order ranging from 1 to 3 (order), if the GU belongs to a main or minor stem (main) and clonal affiliation (clone). The colon sign stands for the interaction of two variables. The parameterized model function is given in Equation 3.10 .

\begin{tabular}{|c|c|c|c|c|c|}
\hline \multirow{2}{*}{$\frac{\text { Model (link) }}{\text { neg. bin. (log) }}$} & $\hat{\theta}(s e)$ & $\begin{array}{ll}l l & d f_{\text {Null }}\end{array}$ & $D_{\text {Model }} \quad d f_{t}$ & $d f_{\text {Model }}$ & $R T$ \\
\hline & $11.04(0.73) \quad 5678$ & $\begin{array}{ll}.1 & 1937\end{array}$ & 2142.6 & $1927 \quad 1$ & $939.3<0.0$ \\
\hline & Covariate & Est. & se & $z$ & $p$ \\
\hline Inte & cept & 0.38812 & 0.06200 & 6.3 & $<0.001$ \\
\hline$l M o$ & & 0.00045 & 0.00002 & 21.0 & $<0.001$ \\
\hline relp & & 1.32767 & 0.05123 & 25.9 & $<0.001$ \\
\hline orde & & -0.63084 & 0.03666 & -17.2 & $<0.001$ \\
\hline orde & & -0.49667 & 0.58551 & -0.8 & 0.396 \\
\hline main & TRUE & 1.01681 & 0.06034 & 16.9 & $<0.001$ \\
\hline clon & $H Y B$ & 0.26580 & 0.06650 & 4.0 & $<0.001$ \\
\hline clon & $A F 2$ & 0.24660 & 0.06205 & 4.0 & $<0.001$ \\
\hline$l M o$ & her : mainTRUE & -0.00024 & 0.00002 & -10.7 & $<0.001$ \\
\hline$l M O$ & her : clone $H Y B$ & -0.00014 & 0.00002 & -5.6 & $<0.001$ \\
\hline$l M o$ & her : clone $A F 2$ & -0.00007 & 0.00003 & -2.9 & 0.004 \\
\hline
\end{tabular}

$\widehat{\eta_{\text {iNodeN }}}=\log \left(\widehat{\mu_{\text {iNodeN }}}\right)=0.38812+$

$$
\begin{aligned}
& \left(0.00045-0.00024 * \text { main }+\left(\begin{array}{c}
0 \\
-0.00014 \\
-0.00007
\end{array}\right) * \text { clone }\right) * \text { lMother } \\
& +1.32767 * \text { relPos }+\left(\begin{array}{c}
0 \\
-0.63084 \\
-0.49667
\end{array}\right) * \text { order }+1.01681 * \text { main } \\
& +\left(\begin{array}{c}
0 \\
0.26580 \\
0.24660
\end{array}\right) * \text { clone }
\end{aligned}
$$


The model captures the positive relationship between the response and the independent variables relPos, lMother and main quite well. Again, it must be advised that relPos was entered as a ratio here and not as \%-values. While the intercept is significantly increased on the main stem overall and for clones 'Hybride 275 ' and 'AF2', the positive slope for lMother is reduced by the interaction terms with clone and main. The positive coefficient for relPos confirms the observations made in Figure 3.23. With increasing branching order the response of the link function and hence the internode count are reduced. The reduction for the third order is smaller than for the second branch order but the few observations in the former must be considered which are also indicated by the comparably large standard error for this factor level.

\subsubsection{Length of Growth Units and Internodes}

Having estimated the number of internodes per growth unit, the focus shifts to inferring the shoot and internode length from the internode count.

In Figure 3.25 the correlation between the internode count and the growth unit length can bee seen. As expected, these variables are positively correlated. The relationship seems to be slightly nonlinear, therefore the internode count was added as a fourth-degree polynomial term without the intercept into the model. Leaving the intercept in the model lead to an unfavorable fitting in the lower value range of the independent variable internode count $i N o d e N$, meaning that negative length estimates would occur for internode counts of 1 or 2 . Omitting an intercept is subject of a controversial discussion (Eisenhauer, 2003). Since dropping the constant from the regression model did not lead to a substantially poorer fit and produces reliable estimates inside the whole range of values for the independent variable, it seemed justifiable here. Using lower degree polynomials lead to undesirable fitting results which would overestimate in the upper value range (second-degree) or yield a parameterized function with a vertex within the value range leading to decreasing estimates (third-degree). Alternatively, two other approaches with a linearized Petterson/Näslund function (as in Schmidt et al., 2011) and nonlinear growth functions (Gompertz/logistic, as in Ritz and Streibig, 2009) were tested. This did not yield satisfying results and will not be further discussed here. All these approaches were aimed at reproducing a sigmoid relationship be- 
tween the two variables which itself is debatable. In Figure 3.25 the increase of $l$ with $i N o d e N$ is dropping beyond $i N o d e N=75$. This seems like extrapolation or at least like an assumption on a weak basis since there are few observations beyond this point. Parameterizing the model function like this and using it within the GrolMP Model leads to more reliable results and seems biologically more plausible. Adding the factor clone to the model led to minimal improvement that was neglected here. Also, adding interaction terms for clone produced an undesirable fitting.

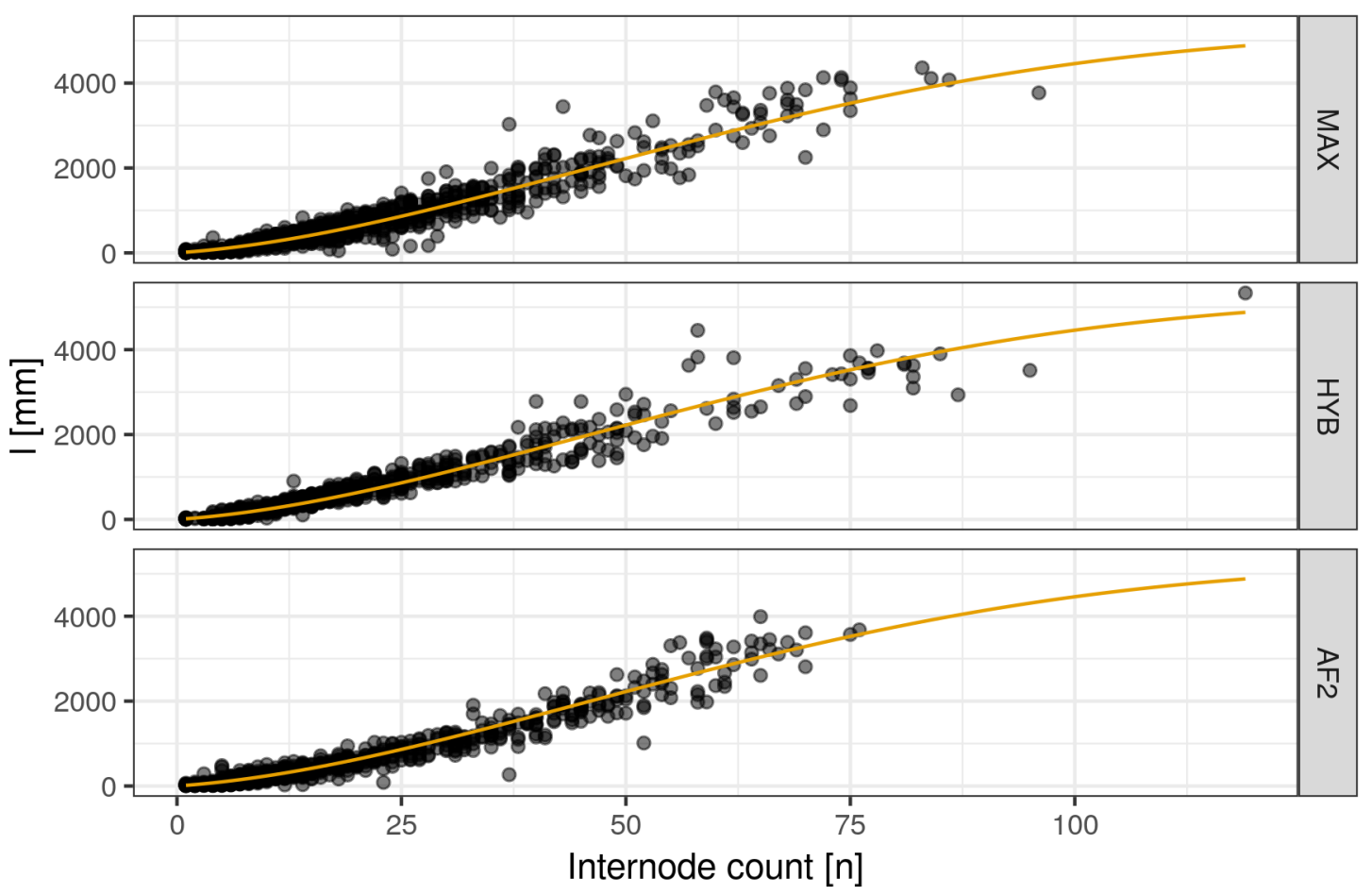

Figure 3.25: Correlation of internode count and growth unit length. The curve is based on the linear model fit.

The summary statistics for the linear model are given in Table 3.16. Since the model has been fit without an intercept, the $F$-test is applied to a comparison with a zero-mean model and instead of the $R_{a d j}^{2}$ the $\operatorname{cor}^{2}(\hat{y}, y)$ is given here. With a value of 0.94 the latter is evaluated as high. The residual standard error of 185.18 seems acceptable interpreted as $\mathrm{mm}$ from a practical viewpoint. 
Table 3.16: Summary of linear model fit for estimating the GU length $l$. Independent variable is the number of internodes in $N$ entered as a fourthdegree polynomial without an intercept.

\begin{tabular}{ccrcrcrr}
\hline$\hat{\sigma}$ & $R S S_{\text {Null }}$ & $d f_{\text {Null }}$ & $R S S_{\text {Model }}$ & $d f_{\text {Model }}$ & $F$ & $p$ & $\operatorname{cor}^{2}(\hat{y}, y)$ \\
\hline 185.18 & 2853555071.1 & 3013 & 103187139.4 & 3009 & 20050.6 & $<0.001$ & 0.94 \\
\hline
\end{tabular}

\begin{tabular}{lrrrc}
\hline Covariate & \multicolumn{1}{c}{ Est. } & \multicolumn{1}{c}{$s e$} & \multicolumn{1}{c}{$t$} & $p$ \\
\hline$i$ NodeN & 14.54898 & 0.85815 & 17.0 & $<0.001$ \\
$i$ NodeN & 1.03103 & 0.05850 & 17.6 & $<0.001$ \\
$i$ NodeN & -0.00999 & 0.00114 & -8.8 & $<0.001$ \\
$i$ NodeN & 0.00003 & 0.00001 & 4.1 & $<0.001$ \\
\hline
\end{tabular}

For estimating the length values, going from GU level to internode level, the relationship between relative proportion of cumulative length to GU length $\frac{i n L}{l}$ and relative internode position relIN was examined (see Figure 3.26). Using these variables leads to more stable results than estimating the internode length directly by the GU's length and the internode's relative rank. A third-degree polynomial without an intercept was fitted to the data. The function process implies shorter internode lengths below a relative rank of $25 \%$, then almost constant lengths that decrease again towards the tip above $75 \%$. The fact that there are "bin"-artifacts visible for some relative node rank's originates from shorter shoots which occur more often and have less varying relative node rank values. This also has an impact on the internode lengths since the steps for relative node ranks get wider and begin with higher values. For example, the first internode of a GU with 4 internodes has a relative rank of $25 \%$ while the first internode of a GU with 20 internodes has a relative rank of $5 \%$. This leads to more evenly spaced internodes for shorter growth units which actually confirms observations within the field.

The summaries for the linear model are presented in Table 3.17. Adding clonal influence or other covariates to the model leads to negligible improvements. The predictive power is very high with $\operatorname{cor}^{2}(y, \hat{y})=0.95$. A value of 0.07 for the residual standard error seems again acceptable. Attention has to be given to the fact that the relative internode rank relI Node was added as a ratio and not as \%-values. 


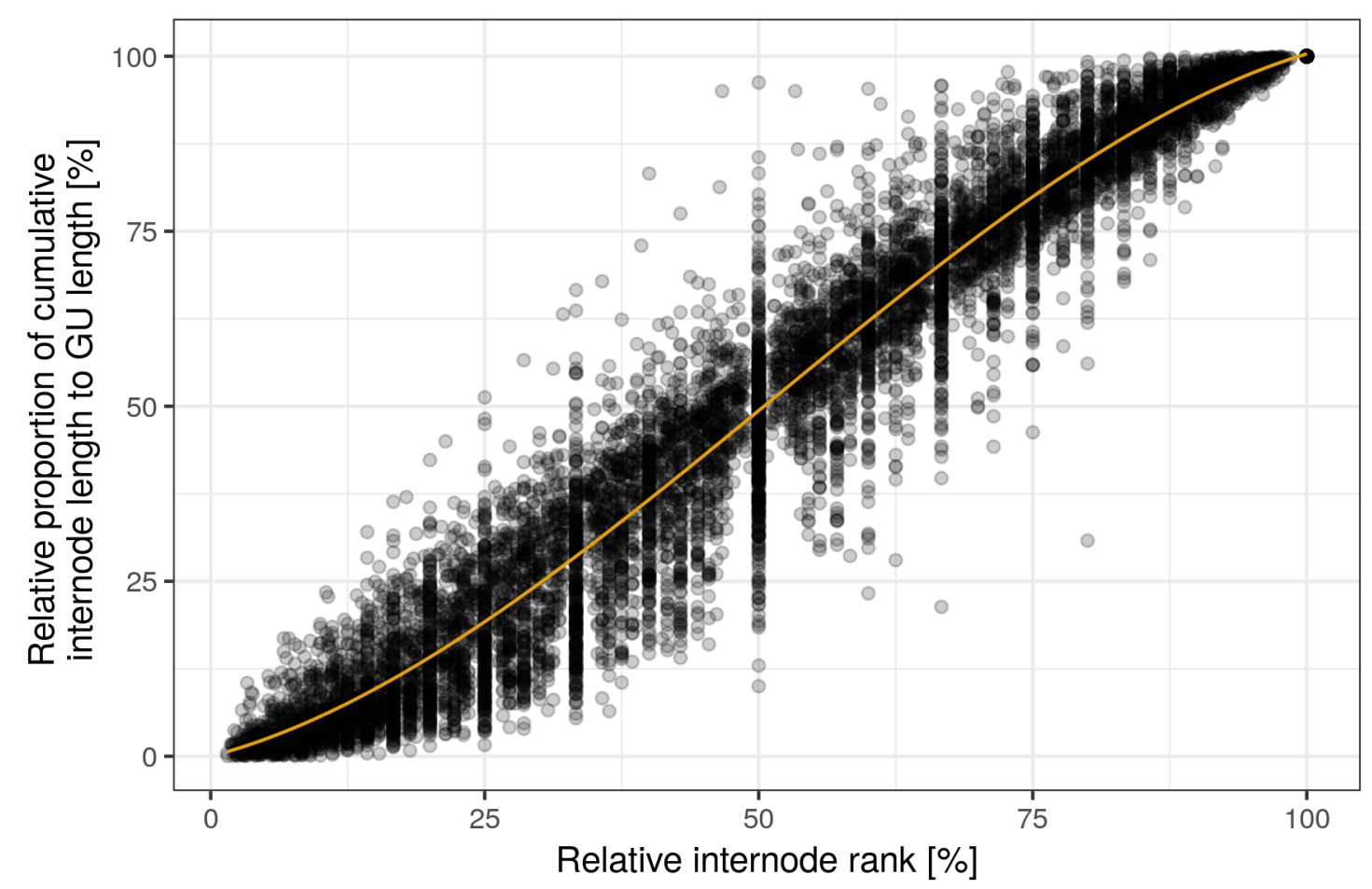

Figure 3.26: Ratio of cumulative internode length to overall growth unit length in relationship to the relative internode rank. The yellow line resembles the thirddegree polynomial linear model fit.

Table 3.17: Summary of linear model fit for estimating the ratio of cumulative internode length to shoot length $\frac{i n L C u m}{l}$. Independent variable is the relative rank of the internode relNode entered as a third-degree polynomial without an intercept.

\begin{tabular}{crrrrccr}
\hline$\hat{\sigma}$ & $R S S_{\text {Null }}$ & $d f_{\text {Null }}$ & $R S S_{\text {Model }}$ & $d f_{\text {Model }}$ & $F$ & $p$ & $\operatorname{cor}^{2}(\hat{y}, y)$ \\
\hline 0.07 & 4059.7 & 10292 & 56.9 & 10289 & 241099.5 & $<0.001$ & 0.95 \\
\hline
\end{tabular}

\begin{tabular}{lrrrc}
\hline Covariate & Est. & se & \multicolumn{1}{c}{$t$} & $p$ \\
\hline relINode & 0.40910 & 0.01213 & 33.7 & $<0.001$ \\
relINode $^{2}$ & 1.71969 & 0.03631 & 47.4 & $<0.001$ \\
relINode $^{3}$ & -1.12533 & 0.02592 & -43.4 & $<0.001$ \\
\hline
\end{tabular}




\subsubsection{Branch Orientation and Directional Angle}

Determining the variables that influence the orientation or branching angle $W$, the variable selection procedure points towards the GU's length $l$, the year in which the mother GU lengthened $t$ Mother, the order of the lateral branch order, whether the GU is part of a main stem or not main and clonal affiliation clone. Figure 3.27 shows the relationship between $W$ and the GU's length. There seems to be a negative trend for $W$ with increasing GU length $l$. The variance seems to decrease also but the lower number of observations needs to be minded.

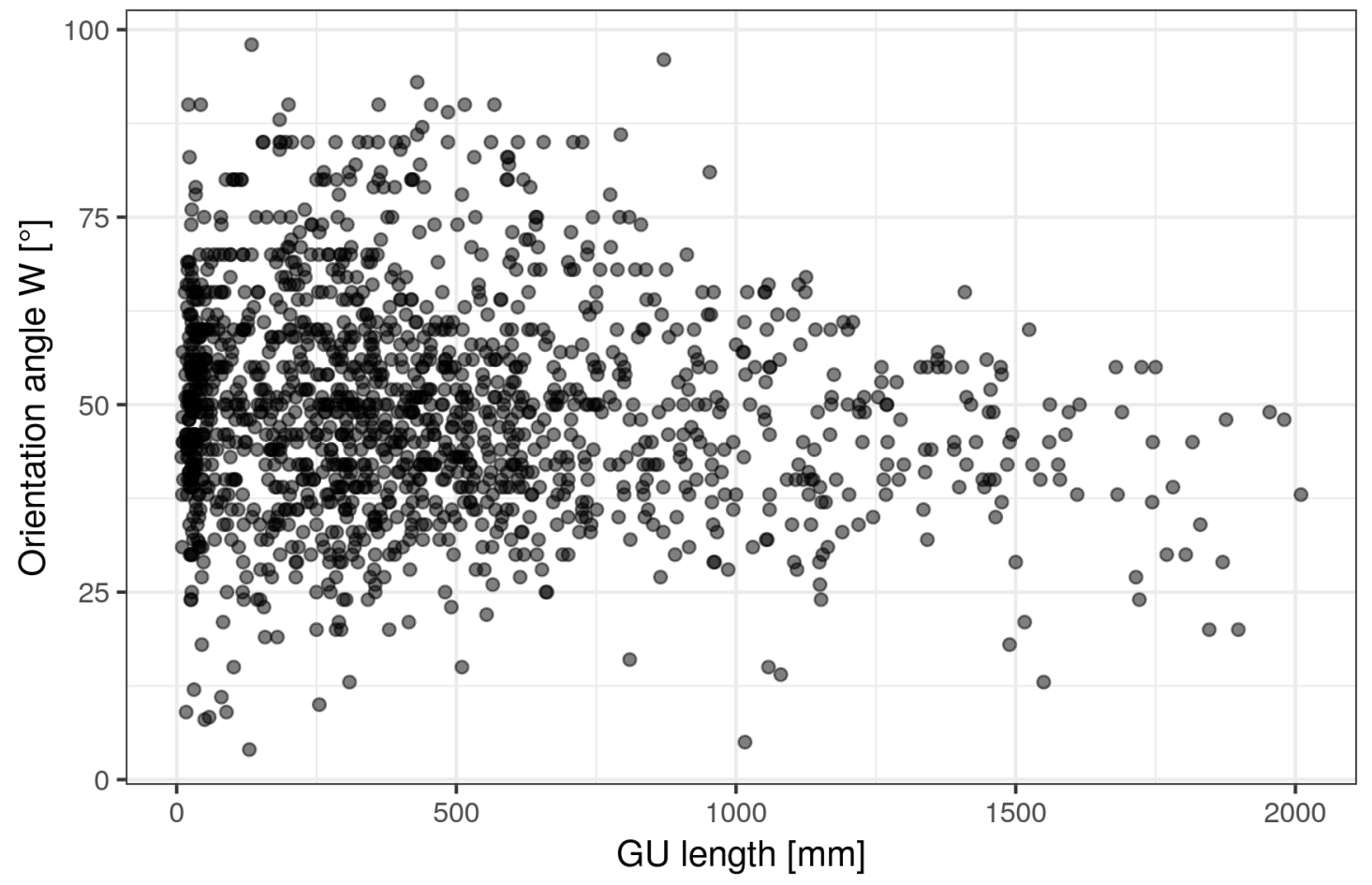

Figure 3.27: Relationship of the orientation angle $W$ and the GU's length $l$.

Figure 3.28 captures the differences in distribution of $W$ by clone and main. The differences by clone are not too distinct. 'Hybride 275' exhibits the largest branch angle values. For all 3 clones there are differences between main and minor stems with the main stem having larger angles. The differences between main and minor differ themselves between the clones which is why the interaction effect of clone : main was kept in the model. 

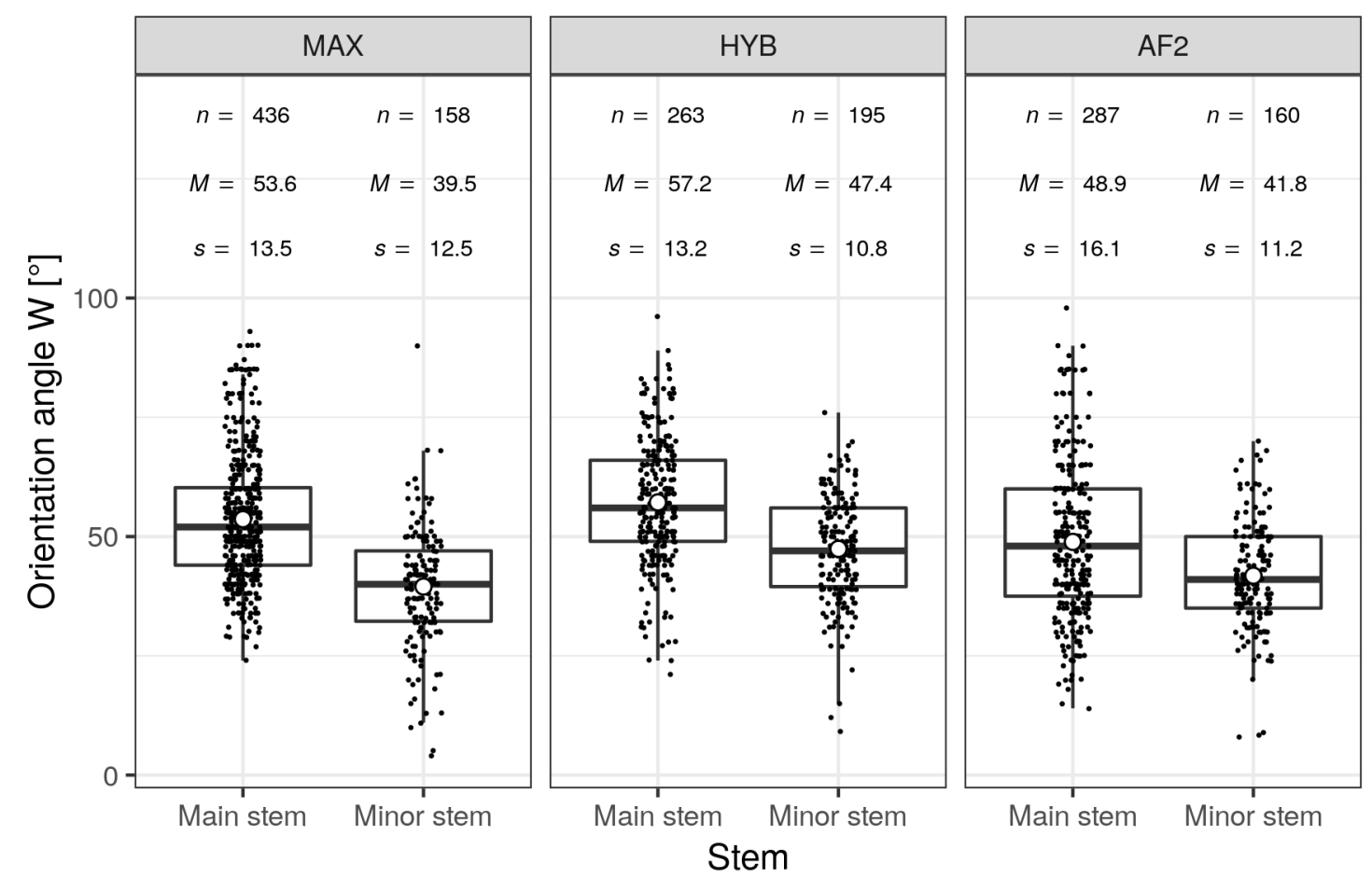

Figure 3.28: Distribution of orientation angle $W$ grouped by clone and whether the $\mathrm{GU}$ is part of a main or minor stem.

These described trends are captured quite well by the linear model (see Table 3.18). Furthermore, the independent variable order has a negative trend with increasing order while tMother has an increase associated to it. Though the model is significantly better than the mean model, the overall predictive power is low with $R_{a d j}^{2}=0.32$ but could not be further improved. 
Table 3.18: Summary of linear model fit for estimating the branch orientation angle $W$. Independent variables are the length of the branch GU $l$, the year that the mother GU was developed in $t$ Mother, the order of the branch order, whether the branch was part of a main or minor stem main and clonal affiliation clone. The colon sign stands for an interaction of two variables. The parameterized model function is given in Equation 3.11.

\begin{tabular}{|c|c|c|c|c|c|c|}
\hline$\hat{\sigma}$ & $R S S_{\text {Null }}$ & $d f_{\text {Null }}$ & $R S S_{\text {Model }}$ & $d f_{\text {Model }}$ & $p$ & $R_{a d j}^{2}$ \\
\hline 11.96 & 315945.1 & 1498 & 212991.2 & 148980. & $0<0.00$ & $01 \quad 0.32$ \\
\hline \multicolumn{3}{|c|}{ Covariate } & Est. & se & $t$ & $p$ \\
\hline \multirow{2}{*}{\multicolumn{3}{|c|}{ Intercept }} & 32.57173 & 31.38497 & $23.5<$ & $<0.001$ \\
\hline & & & -0.01173 & \multirow{2}{*}{$\begin{array}{ll}3 & 0.00098 \\
2 & 0.76548\end{array}$} & $-12.0<$ & $<0.001$ \\
\hline \multicolumn{3}{|c|}{ tMother } & 10.39462 & & $13.6<$ & $<0.001$ \\
\hline \multicolumn{3}{|c|}{ order 2} & -13.82286 & $6 \quad 0.97711$ & $-14.1<$ & $<0.001$ \\
\hline \multicolumn{3}{|c|}{ order 3} & -26.05852 & 5.02809 & $-5.2<$ & $<0.001$ \\
\hline \multicolumn{3}{|c|}{$\operatorname{mainTRUE}$} & 15.71190 & 1.22592 & $12.8<$ & $<0.001$ \\
\hline \multicolumn{3}{|c|}{ clone $H Y B$} & 5.84895 & 1.28502 & $4.6<$ & $<0.001$ \\
\hline \multicolumn{3}{|c|}{ clone $A F 2$} & 2.72268 & 1.36802 & 2.0 & 0.047 \\
\hline \multirow{2}{*}{\multicolumn{3}{|c|}{ mainTRUE : clone $H Y B$}} & -3.72289 & 1.59438 & -2.3 & 0.02 \\
\hline \multicolumn{2}{|c|}{ mainTRUE : clone AF2 } & & -11.26298 & 1.66762 & $-6.8<$ & $<0.001$ \\
\hline
\end{tabular}

$$
\begin{aligned}
\widehat{W}= & 32.57173-0.01173 * l+10.39462 * \text { tMother }+\left(\begin{array}{c}
0 \\
-13.82286 \\
-26.05852
\end{array}\right) * \text { order } \\
& +\left(15.71190+\left(\begin{array}{c}
0 \\
-3.72289 \\
-11.26298
\end{array}\right) * \text { clone }\right) * \text { main }+\left(\begin{array}{c}
0 \\
5.84895 \\
2.72268
\end{array}\right) * \text { clone }
\end{aligned}
$$

Modeling the differences of consecutive directional angles of lateral branches and, since these develop from axillary buds of leaves (Bartels, 1987), of leaves also, did not point towards any influential variables that were plausible except clone. In Table 3.19 some summaries for these differences are displayed. Problematic with calculating these differences is that with the manual measuring, which was used here only, the directional angle $R$ was only measured for lat- 
eral branches and not for "empty" nodes without lateral shoots. So the resulting angle differences do not automatically correspond to the phyllotactic pattern.

Table 3.19: Summary data for differences in directional angle $R$ between consecutive lateral branches $R$ Diff separated by clone. Displayed are the sample size $\mathrm{n}$, the arithmetic mean $M$, the median $M d n$ and the sample standard deviation $s$.

\begin{tabular}{lcccc}
\hline Clone & $\mathrm{n}$ & $M\left[^{\circ}\right]$ & $M d n\left[^{\circ}\right]$ & $s\left[^{\circ}\right]$ \\
\hline MAX & 755 & 143.34 & 135.00 & 63.98 \\
HYB & 494 & 159.96 & 135.00 & 59.57 \\
AF2 & 506 & 158.30 & 135.00 & 62.63 \\
\hline
\end{tabular}

The mean values point to some differences. Fitting a linear model with clone as the only independent variable proves to be better than a simple mean model (see Table 3.20). The predictive power is however very poor with $R_{a d j}^{2}=0.01$ and with $\hat{\sigma}=62.38$ the residual standard error is quite large from a practical viewpoint.

Table 3.20: Summary of linear model fit for estimating the directional angle $R$. Independent variable is the clonal affiliation clone.

\begin{tabular}{|c|c|c|c|c|c|c|c|c|}
\hline$\frac{\hat{\sigma}}{62.38}$ & \multirow{2}{*}{$\begin{array}{c}R S S_{\text {Null }} \\
6924720.0\end{array}$} & $d f_{\text {Null }}$ & \multicolumn{2}{|c|}{$R S S_{\text {Model }}$} & \multicolumn{2}{|c|}{$d f_{\text {Model }}$} & $F$ & $p$ \\
\hline \multirow[t]{5}{*}{62.38} & & 1754 & 681696 & 88.0 & 17 & & 13.8 & $<0.00$ \\
\hline & Covariate & & Est. & & $e$ & $t$ & & $p$ \\
\hline & Intercept & & 3.34437 & 2.2 & 015 & 63.1 & & 0.001 \\
\hline & clone $H Y E$ & & 0.61514 & 3.6 & 971 & 4.6 & & 0.001 \\
\hline & clone $A F 2$ & & 4.95602 & & 3375 & 4.2 & & 0.001 \\
\hline
\end{tabular}

Reviewing the summaries in Tables 3.19 and 3.20 the uniform median value of $135^{\circ}$ of all 3 clones stands out. Relating this value to $360^{\circ}$ results in a ratio of $\frac{135}{360}=0.375$ which equals the fraction of a full rotation from leaf to leaf described for poplar by Coxeter (1969). Kadereit et al. (2014) also mention $135^{\circ}$ as a common divergence angle in alternate leaf arrangement. For shortened internodes this arrangement will become more spiral-like (Kadereit et al., 2014) which will later be exemplified for leaves on short shoots (see Section 3.2.9). Therefore the decision was made to set the divergence angle for all clones to $135^{\circ}$. 


\subsubsection{Diameter of Growth Units}

As described in Section 3.1.3, the manually measured GU-wise mid-diameter values where divided by the GU's age to obtain the mean annual diameter increment $d I n c r$. Searching for the variables that are most suitable for estimating the diameter increment, the variable selection indicated the independent variables GU length $l$, rotation year that the GU was developed in $t$, branch order order, whether the GU belonged to a main stem or not main and clonal affiliation clone as important. Figure 3.29 displays the correlation of annual mid-diameter increment with GU length, clone and rotation year.
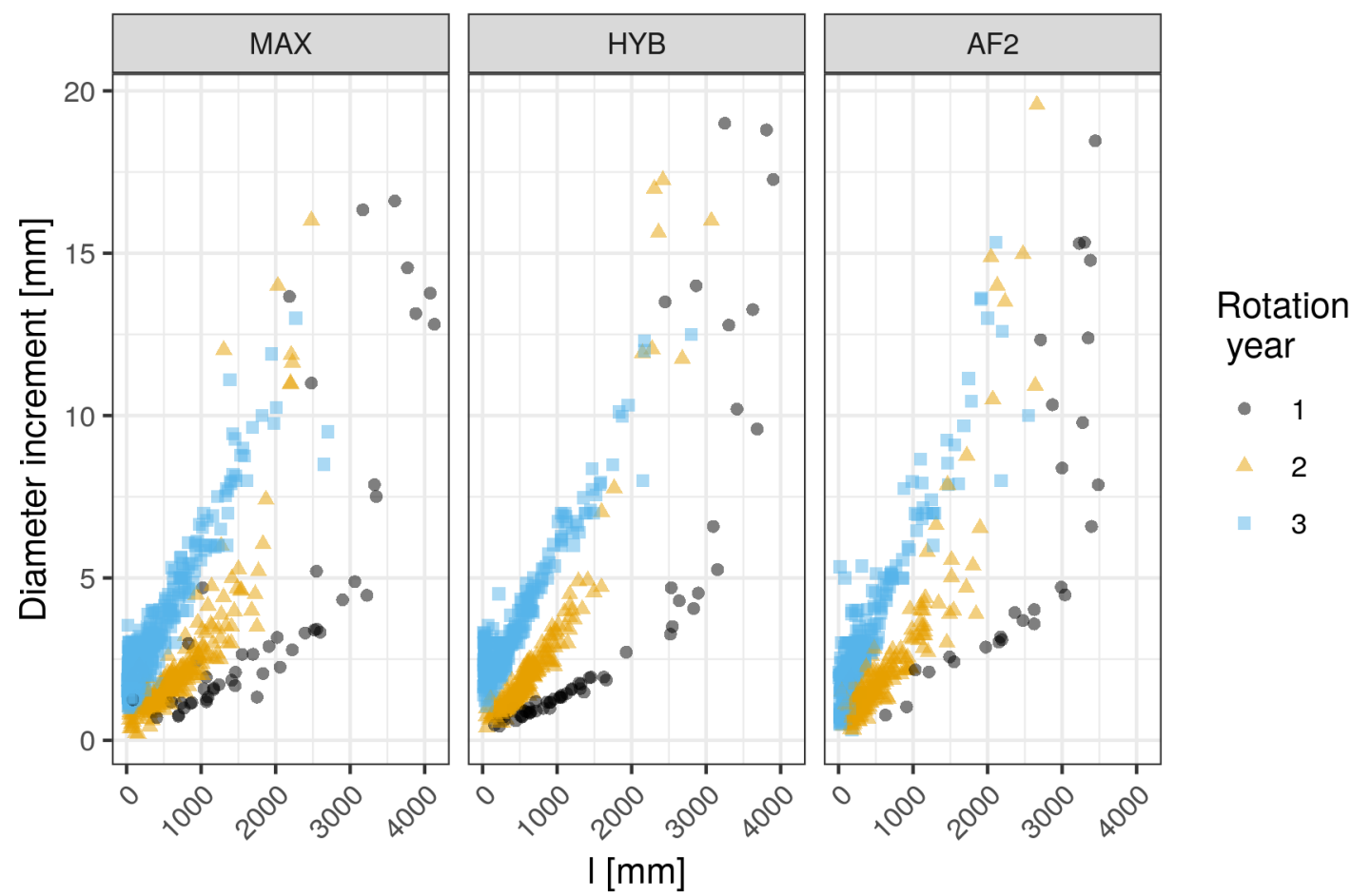

Figure 3.29: Relationship of the GU length $l$ and the mean annual diameter increment $d$ Incr separated by clone in facets and rotation year $t$ marked by color.

The curvature of the relationship between diameter increment and length changes over the rotation years. The oldest shoots from the first year exhibit an exponential increase in diameter increment which is less pronounced for growth units of the second year. For growth units from the third year the relationship is almost linear. Furthermore, for comparable lengths the GUs from the third year show higher increments than those from the second and first year. This points towards 
an uneven distribution of secondary growth. The increased variance for higher diameter increment, which is most distinct for GUs from the first rotation year, followed by the second and third year, originates likely from competition between neighboring trees.

Table 3.21: Summary of linear model fit for estimating the annual diameter increment $d$ Incr. The response was transformed as $d \operatorname{Incr}^{0.3}$. Independent variables are the GU length $l$, the rotation year that the GU was developed in $t$, the branch order order, whether the GU belonged to a main stem or not main and clonal affiliation clone. The colon sign stands for an interaction of two variables. The parameterized model function is given in Equation 3.12 .

\begin{tabular}{|c|c|c|c|c|c|c|}
\hline$\hat{\sigma}$ & $R S S_{\text {Null }} \quad d f$ & ull $\quad R S S_{\text {Model }}$ & $d f_{\text {Model }}$ & $F$ & $p$ & $R_{a d j}^{2}$ \\
\hline 0.11 & 144.7 & 21.4 & 1851 & 821.6 & $<0.001$ & 0.85 \\
\hline & Covariate & Est. & se & $t$ & $p$ & \\
\hline & Intercept & 0.3899780 & 0.016709 & $4 \quad 23.3$ & $<0.001$ & \\
\hline & $l$ & 0.0003744 & 0.000008 & $5 \quad 44.1$ & $<0.001$ & \\
\hline & $t$ & 0.3023874 & 0.005599 & 054.0 & $<0.001$ & \\
\hline & order 1 & -0.1044617 & 0.014187 & -7.4 & $<0.001$ & \\
\hline & order 2 & -0.1552537 & $0.016251 \mathrm{~s}$ & -9.6 & $<0.001$ & \\
\hline & order 3 & -0.1596862 & 0.079595 & -2.0 & 0.045 & \\
\hline & $\operatorname{main} T R U E$ & 0.0506769 & 0.006208 & 8.2 & $<0.001$ & \\
\hline & clone $H Y B$ & 0.0154841 & 0.008407 & 1.8 & 0.066 & \\
\hline & clone $A F 2$ & -0.0323174 & 0.008058 & $-4 . c$ & $<0.001$ & \\
\hline & $l:$ order 1 & 0.0000531 & 0.000010 & 5.2 & $<0.001$ & \\
\hline & $l:$ order 2 & 0.0001511 & 0.000037 & 4.1 & $<0.001$ & \\
\hline & $l:$ order 3 & -0.0011263 & 0.002504 & -0.4 & 0.653 & \\
\hline & $l:$ clone $H Y B$ & 0.0000075 & 0.000009 & $0 . \varepsilon$ & 0.427 & \\
\hline & $l:$ clone $A F 2$ & 0.0000486 & 0.000009 & 5.2 & $<0.001$ & \\
\hline
\end{tabular}




$$
\begin{aligned}
\widehat{d \operatorname{Incr}^{0.3}=} & 0.3899780 \\
& +\left(0.0003744+\left(\begin{array}{c}
0 \\
0.0000531 \\
0.0001511 \\
-0.0011263
\end{array}\right) * \text { order }+\left(\begin{array}{c}
0 \\
0.0000075 \\
0.0000486
\end{array}\right) * \text { clone }\right) * l \\
& +0.3023874 * t+\left(\begin{array}{c}
0 \\
-0.1044617 \\
-0.1552537 \\
-0.1596862
\end{array}\right) * \text { order }+0.0506769 * \text { main } \\
& +\left(\begin{array}{c}
0 \\
0.0154841 \\
-0.0323174
\end{array}\right) * \text { clone }
\end{aligned}
$$

The results of the linear model fit are presented in Table 3.21. A transformation to a power with exponent 0.3 (as deemed suitable by the Box-Cox procedure) was applied to the response. Other variants with adding the length as a polynomial term did not produce satisfying results. As expected, the effects for variables $t$, main and $l$ are estimated as positive. The latter is additionally enhanced for clones 'Hybride 275' and 'AF2' by the $l$ : clone interaction, although for the former clone this is not significantly different from 0 which is also the case for the main clonal effect of 'Hybride 275'. 'AF2' has a significantly lowered intercept in comparison to 'Max 1'. The effects for branch order are increasingly negative although the third branch order did again not test significant with its effect due to the high standard error based on few observations. The interaction of length with branch order is increasingly positive and the third branch order is again not too reliable in its estimation. Overall, the model performed better than the corresponding null model and the overall predictive power is good with $R_{a d j}^{2}=0.85$. 


\subsubsection{Syllepsis}

As described in the Material and Methods Section 3.1, differentiating sylleptic from regular shoots mainly relied on plausibility checks of shoot age and order. For shoots developed in the last vegetation period (from time of measurement) this was comparably simple while identifying sylleptic shoots from previous vegetation periods demanded analyzing the topology. The overall absolute frequency of sylleptic shoots on nodes is low and even lower if this number is related to the number of empty nodes or nodes with regular shoots. By the means of variable selection, 4 variables were identified that were deemed suitable for estimating the probability of a sylleptic shoot occurring. These were main expressing if a node on a main or minor stem was considered, $t$ implying the rotation year, order meaning the branch order and clone standing for genotype. Furthermore, the interaction of main : $t$ yielded a significant effect. This dependency can be quantified in Table 3.22. The count of sylleptic shoots is as said very low, no combination of $t$ and main has a relative frequency $>1 \%$. The number of sylleptic shoots increases with the rotation years. This increase is higher on minor stems while the overall probability is higher on main stems.

Table 3.22: Frequency table for number of sylleptic, lateral shoots versus none or regular shoots grouped by main/minor stem main and rotation year $t$.

\begin{tabular}{llrrr}
\hline main & $t$ & Total [n] & Sylleptic shoots [n] (\%) & None or regular shoots [n] (\%) \\
\hline minor & 1 & 5506 & $2(0.04 \%)$ & $5504(99.96 \%)$ \\
minor & 2 & 9841 & $0(0 \%)$ & $9841(100 \%)$ \\
minor & 3 & 3566 & $17(0.48 \%)$ & $3549(99.52 \%)$ \\
main & 1 & 1323 & $0(0 \%)$ & $1323(100 \%)$ \\
main & 2 & 11988 & $33(0.28 \%)$ & $11955(99.72 \%)$ \\
main & 3 & 14280 & $28(0.2 \%)$ & $14252(99.8 \%)$ \\
\hline
\end{tabular}

Table 3.23 contains the absolute frequencies of sylleptic shoots grouped by the other two independent variables. With increasing branch order the probability for sylleptic shoots decreases, although this trend eventuates on a very low level. The probabilities by clone are comparably minor although there are significant differences by clone with 'AF2' having the highest probability. 
Table 3.23: Frequency table for number of sylleptic, lateral shoots versus none or regular shoots grouped by branch order order and clonal affiliation clone.

\begin{tabular}{ll|r|rr}
\hline Variable & Value & Total [n] & Sylleptic shoots [n] (\%) & None or regular shoots [n] (\%) \\
\hline order & 0 & 10962 & $58(0.53 \%)$ & $10904(99.47 \%)$ \\
order & 1 & 30943 & $20(0.06 \%)$ & $30923(99.94 \%)$ \\
order & 2 & 4591 & $2(0.04 \%)$ & $4589(99.96 \%)$ \\
order & 3 & 8 & $0(0 \%)$ & $8(100 \%)$ \\
clone & MAX & 17942 & $9(0.05 \%)$ & $17933(99.95 \%)$ \\
clone & HYB & 14425 & $21(0.15 \%)$ & $14404(99.85 \%)$ \\
clone & AF2 & 14137 & $50(0.35 \%)$ & $14087(99.65 \%)$ \\
\hline
\end{tabular}

While the parameterized generalized linear model captures the described relations quite well (see Table 3.24), the overall predictive power, besides the model being significantly better than the null model, is low with $D_{a d j}^{2}=0.21$.

Table 3.24: Summary of generalized linear model fit for estimating $\eta$ of the response hasSyll of sylleptic lateral shoots occurring (1) vs regular, lateral or no shoots occurring (0). Variable $t$ resembles the rotation year in which the lateral shoot elongates, order the branch order of the lateral shoot and clone the clonal affiliation. The colon sign stands for the interaction of two variables. The parameterized model function is given in Equation 3.14.

\begin{tabular}{|c|c|c|c|c|c|c|}
\hline \multirow{2}{*}{$\frac{\text { Model (link) }}{\text { q.-binom. (logit) }}$} & $\Phi$ & \multirow{2}{*}{$\frac{D_{\text {Null }}}{1178.3}$} & \multirow{2}{*}{$\begin{array}{c}d f_{\text {Null }} \\
46503\end{array}$} & \multicolumn{2}{|c|}{$D_{\text {Model }} \quad d f_{\text {Model }}$} & $p$ \\
\hline & 1.64 & & & $934.1 \quad 46$ & 9518 & $8.6<0.001$ \\
\hline \multicolumn{2}{|c|}{ Covariate } & \multicolumn{2}{|r|}{ Est. } & se & $t$ & $p$ \\
\hline \multirow{2}{*}{\multicolumn{2}{|c|}{$\begin{array}{l}\text { Intercept } \\
\text { mainT RUE }\end{array}$}} & \multicolumn{2}{|c|}{-13.06779} & 1.82594 & -7.2 & $<0.001$ \\
\hline & & & .14035 & 1.88432 & 3.3 & 0.001 \\
\hline \multicolumn{2}{|l|}{. } & & .76740 & 0.63300 & 4.4 & $<0.001$ \\
\hline \multicolumn{2}{|c|}{ order 1} & & .94229 & 0.33842 & -8.7 & $<0.001$ \\
\hline \multicolumn{2}{|c|}{ order 2} & & .95142 & 0.93085 & -4.2 & $<0.001$ \\
\hline \multicolumn{2}{|c|}{ order 3} & & .58723 & 659.57561 & -0.0 & 0.985 \\
\hline \multicolumn{2}{|c|}{ clone $H Y B$} & & .92892 & 0.51259 & 1.8 & 0.07 \\
\hline \multicolumn{2}{|c|}{ clone AF 2} & & .78675 & 0.46603 & 3.8 & $<0.001$ \\
\hline \multicolumn{2}{|c|}{$\operatorname{mainTRUE}: t$} & & .12902 & 0.67544 & -3.2 & 0.002 \\
\hline
\end{tabular}




$$
\begin{aligned}
\hat{\eta}=\log \left(\frac{p}{1-p}\right)= & -13.06779+6.14035 * \text { main }+(2.76740-2.12902 * \text { main }) * t \\
& +\left(\begin{array}{c}
0 \\
-2.94229 \\
-3.95142 \\
-12.58723
\end{array}\right) * \text { order }+\left(\begin{array}{c}
0 \\
0.92892 \\
1.78675
\end{array}\right) * \text { clone }
\end{aligned}
$$

The differences for the third branch order from order 0 are not significant, due to the high standard error. The differences between 'Max 1' and 'Hybride 275' are not significant either while the standard error has a reasonable size.

One additional fact that needs consideration and which came up during the model building in GrolMP was that no sylleptic branch has any sylleptic ancestors. That a sylleptic shoot can not have a direct sylleptic successor is trivial but the possibility of a sylleptic shoot having a regular, lateral successor which on its own has another sylleptic successor needed to be ruled out. Not taking this into account lead to problems with functions that incorporate for example an effect for order because branch orders would be reached that were not accounted for in the models. The probability for such a scenario to happen is highly unlikely due to the low probability of sylleptic branches to develop. Since it is not impossible, the topology for all sylleptic branches was analyzed if any case of a sylleptic branch having some sylleptic ancestor would exist. Owing to the fact that no occasions were found, this possibility was ruled out in structural modeling.

From establishing the probability of a sylleptic shoot developing, the focus shifts to the number of internodes of these GUs. Figure 3.30 displays the correlation of number of internodes $i N o d e N$ by length of mother GU lMother for sylleptic GUs.

The correlation is clearly positive and nonlinear. By variable selection the independent variable $l$ Mother was regarded as sufficient which is also resembled by the high $D_{a d j}^{2}$ of 0.8 for the fitted generalized linear model (see summaries in Table 3.25. 


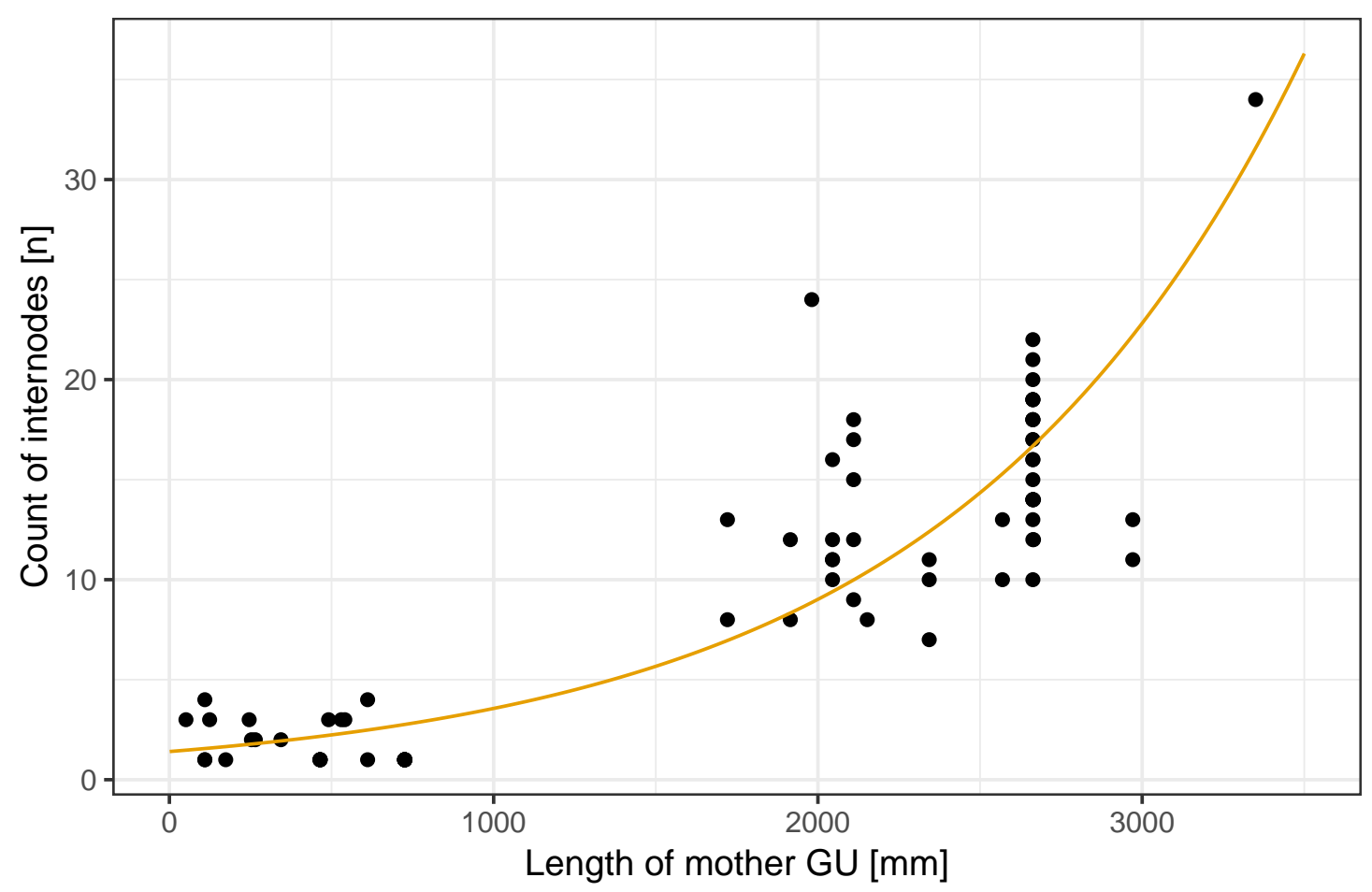

Figure 3.30: Relationship between number of internodes and length of mother GU for sylleptic shoots. The yellow line is the back-transformed response from the generalized linear model.

Table 3.25: Summary of generalized linear model fit for estimating $\eta$ of the response innSyll of number of internodes of sylleptic lateral shoots. Variable lMother resembles the length of the mother GU.

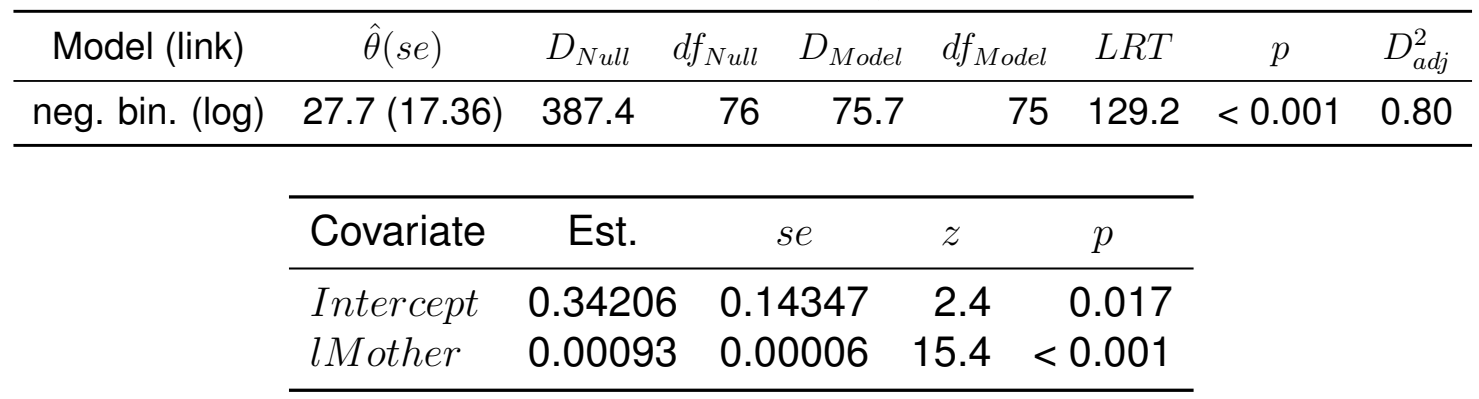


All other morphological parameters of sylleptic shoots are estimated with the same models as for the regular shoots. The differences seemed either negligible or are already accounted for by the dependencies of most models on shoot length determined by internode count.

\subsubsection{Branch Curvature}

After importing the 3D curvature data into $R$, some of the data sets still had to be merged via Procrustes analysis (as described in 3.1.4). 39 of these analyses were performed and resulted in an average value for Ordinary Procrustes sum of squares (OSS) of 6.56 with a value of 7.01 for standard deviation. The mean for the root mean square deviation was 0.82 with a standard deviation of 0.49 . These values are in the $\mathrm{cm}$ scale since the curvature coordinates were transformed to $\mathrm{mm}$ after Procrustes analysis. Nevertheless, a value of below $1 \mathrm{~cm}$ for, simplified, a measure of the average distance between corresponding connection points from both data sets, can be seen as a good result. A summary of the goodness of fit in the form of proportional variance explained by each principal component $(\mathrm{PC})$ is given in Figure 3.31. The overall impression is very good: For all cases the average proportion of variance explained by the first PC is $98.13 \%$ with a standard deviation of $2.46 \%$. The 2 nd PC was hence much lower with a mean of $1.73 \%$ and a standard deviation of $2.26 \%$. On average $0.14 \%$ of the variance could be explained by the 3rd PC with a much lower standard deviation of $0.37 \%$. This points towards the fact that the branch-wise data structure of the 3D coordinates could quite well by explained the first two principal components and therefore be projected from $3 D$ to $2 D$ space without too much loss of information.

After applying the dimensional reduction and translating/rotating the 2D branch coordinates, simple shape parameters were calculated as described in 3.1.4. In Figure 3.32 the ratios $\frac{b}{l}$ and $\frac{l_{m}}{l}$ are set in relation to the chord length $l$ as the straight distance between each branch's base and its tip. Both relationships were further scrutinized by linear modeling. Width to length as ratio $\frac{b}{l}$ is significantly influenced by clonal affiliation only. The mean value for 'Max 1 ' is 0.09 , for 'Hybride 275' 0.08 and for 'AF2' 0.12 . The latter clone, therefore, exhibits a slightly more distinctive branch curvature. The average relative position of the maximum 


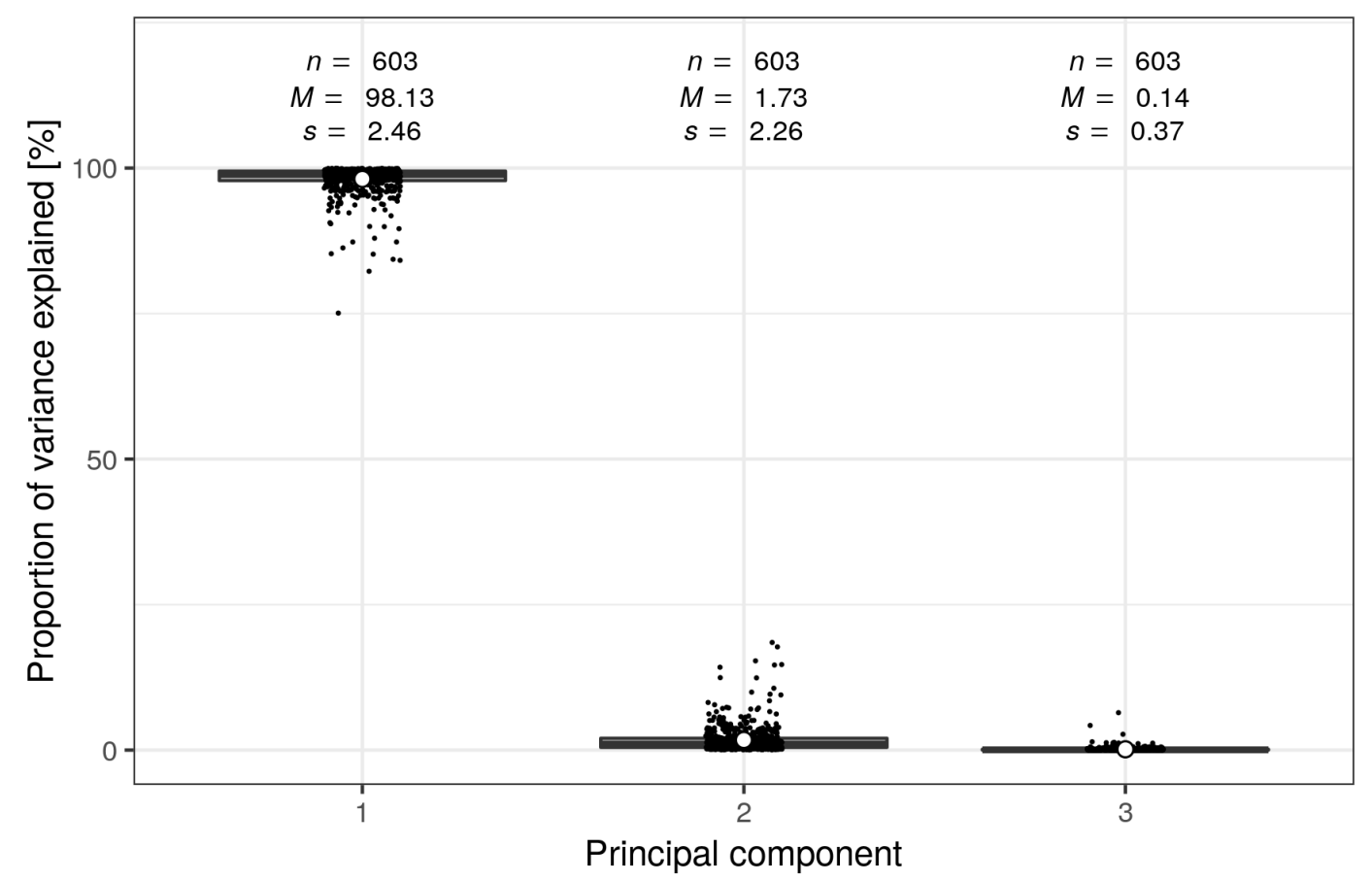

Figure 3.31: Proportion of variance explained per principal component in principal component analysis for dimensional reduction in projecting node coordinates from $3 \mathrm{D}$ to $2 \mathrm{D}$.

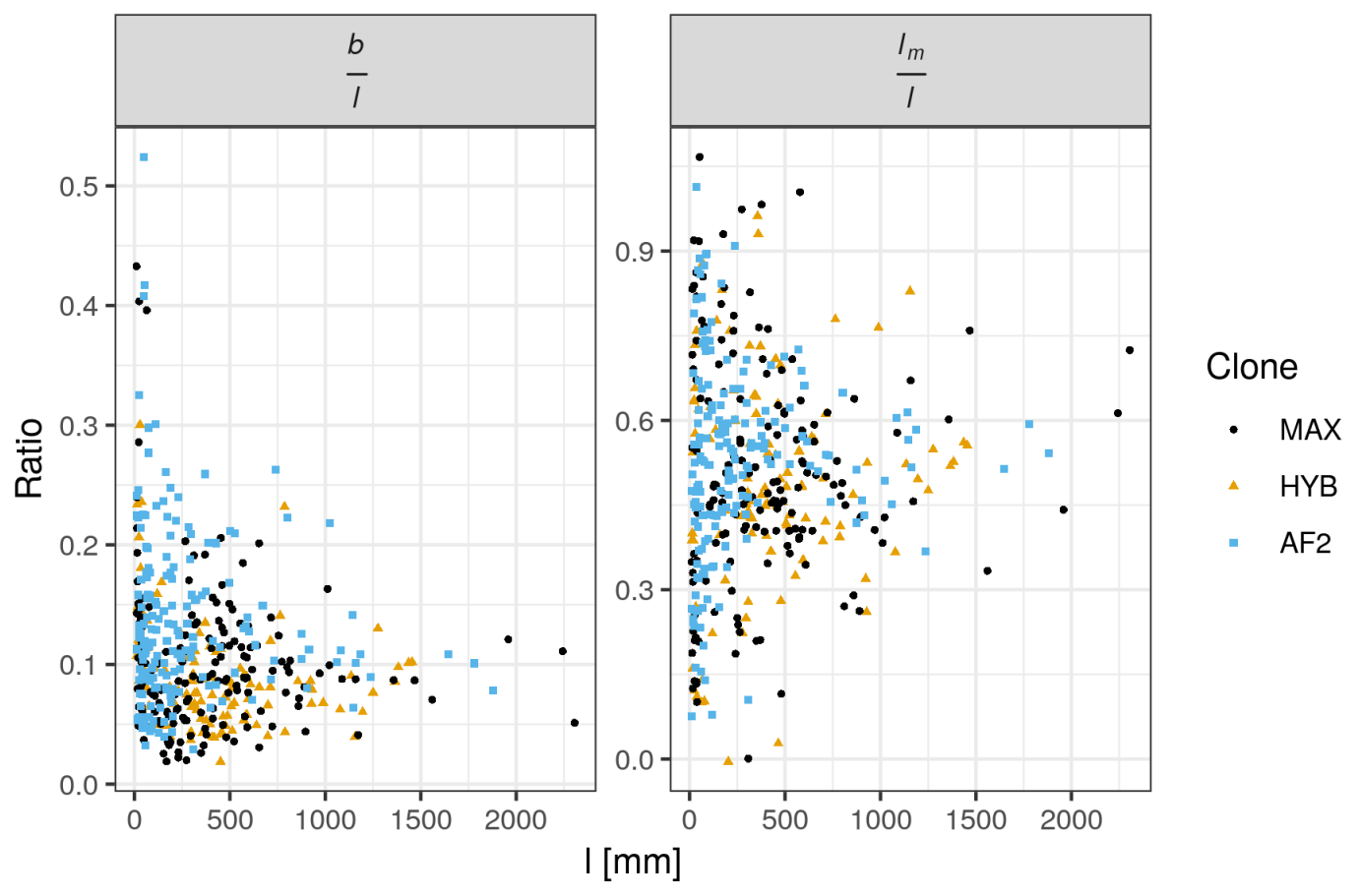

Figure 3.32: Distribution of the values of two ratios of maximum width $b$ to chord length $l$ and vertical position of maximum width $l_{m}$ to chord length $l$. Different colors and shapes represent the clonal affiliation per observation. 
width per growth unit $\frac{l_{m}}{l}$ was not significantly influenced by the variables tested. The overall mean for all clones is 0.51 .

After examining the shape parameters, a 2nd-degree polynomial was fit to the $2 \mathrm{D}$ coordinates of each growth unit to approximate the branch curvature. As a part of this procedure, the 2nd-degree polynomial fit was tested against the null model for all growth units. As stated in section 3.1.4, if based on the $F$-test the null hypothesis could not be rejected the growth unit was assumed not to be curved. The quantity of straight and curved growth units by their arc length is contrasted in Figure 3.33 .

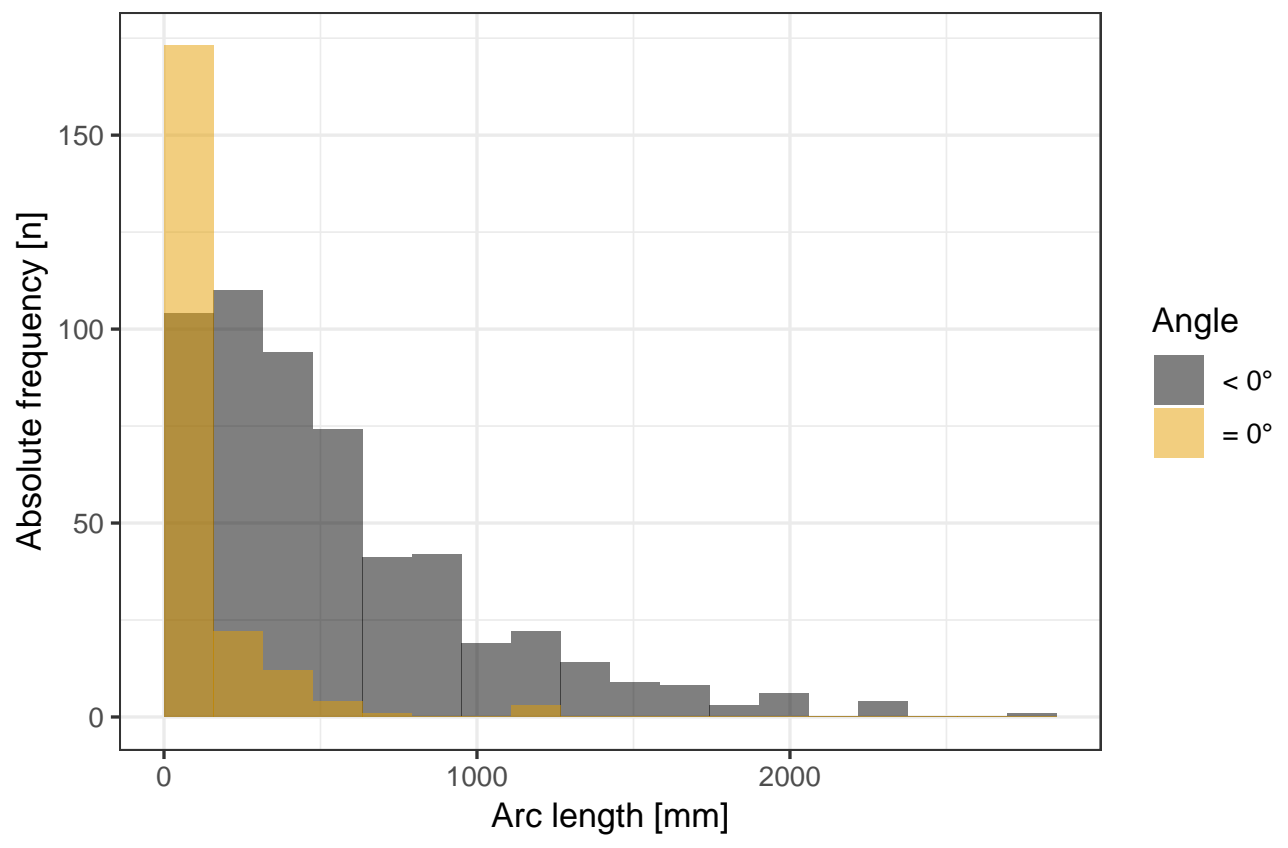

Figure 3.33: Count of straight growth units with no curvature angle $\left(=0^{\circ}\right)$ and of curved growth units $\left(<0^{\circ}\right)$ by the arc length of the growth unit.

Overall, shorter arc lengths occur to a much greater extent than longer lengths. The count of straight growth units is displayed as yellow bins in Figure 3.33. The proportion of these growth units to curved growth units is increasing with decreasing arc length. The longest growth unit that was assumed to be straight was 1250 $\mathrm{mm}$ long. This concurs with observations in the field since smaller and younger growth units often tend to be straight and not curved, as long at least as their elongation is not too advanced.

For the structural model the differentiation between curved and straight growth 
units needed to be further quantified via a generalized linear model. The arc length $l$ turned out to be the only significant and suitable predictor variable for the probability of a curved (1) versus a straight (0) GU. Summaries for this quasibinomial GLM are given in Table 3.26. Some overdispersion was detected, hence the quasi approach. The predictive power evaluable by $D_{a d j}^{2}=0.37$ is intermediate. The positive coefficient for $l$ captures the determined tendency well.

Table 3.26: Summary of generalized linear model fit for estimating $\eta$ of the response isCurved determining if a shoot is assumed to be curved (1) or straight (0). Variable $l$ resembles GU length.

\begin{tabular}{|c|c|c|c|c|c|c|c|c|}
\hline Model (link) & $\Phi$ & $D_{\text {Null }}$ & $d f_{\text {Null }}$ & $D_{\text {Model }}$ & $d f_{\text {Model }}$ & $F$ & $p$ & $D_{a d j}^{2}$ \\
\hline \multirow[t]{3}{*}{ q.-binom. (logit } & 1.88 & 1061.9 & 766 & 666.0 & 765 & 210.4 & $<0.001$ & 0.37 \\
\hline & Covaria & & st. & se & $t$ & $p$ & & \\
\hline & $l$ & 0.0 & 477 & 0.00054 & 8.9 & $<0.001$ & & \\
\hline
\end{tabular}

Focusing now on the curved growth units only, Figure 3.34 summarizes the predictive power for all fitted models that performed significantly better than the null model. The left panel includes the absolute frequency of the squared correlation of the fitted and observed values $\operatorname{cor}^{2}(\hat{y}, y)$.

The distribution is left skewed with the majority of the values above 0.5 . The median value is 0.89 . The right panel shows the absolute frequency of the residual standard error of each model fit. This mirrors the prior findings with a rightskewed distribution. The majority of $\hat{\sigma}$ values is below $10 \mathrm{~mm}$. The median value here is 4.16. Based on the distribution of the values of these parameters the curve approximations can overall be assessed as good. 

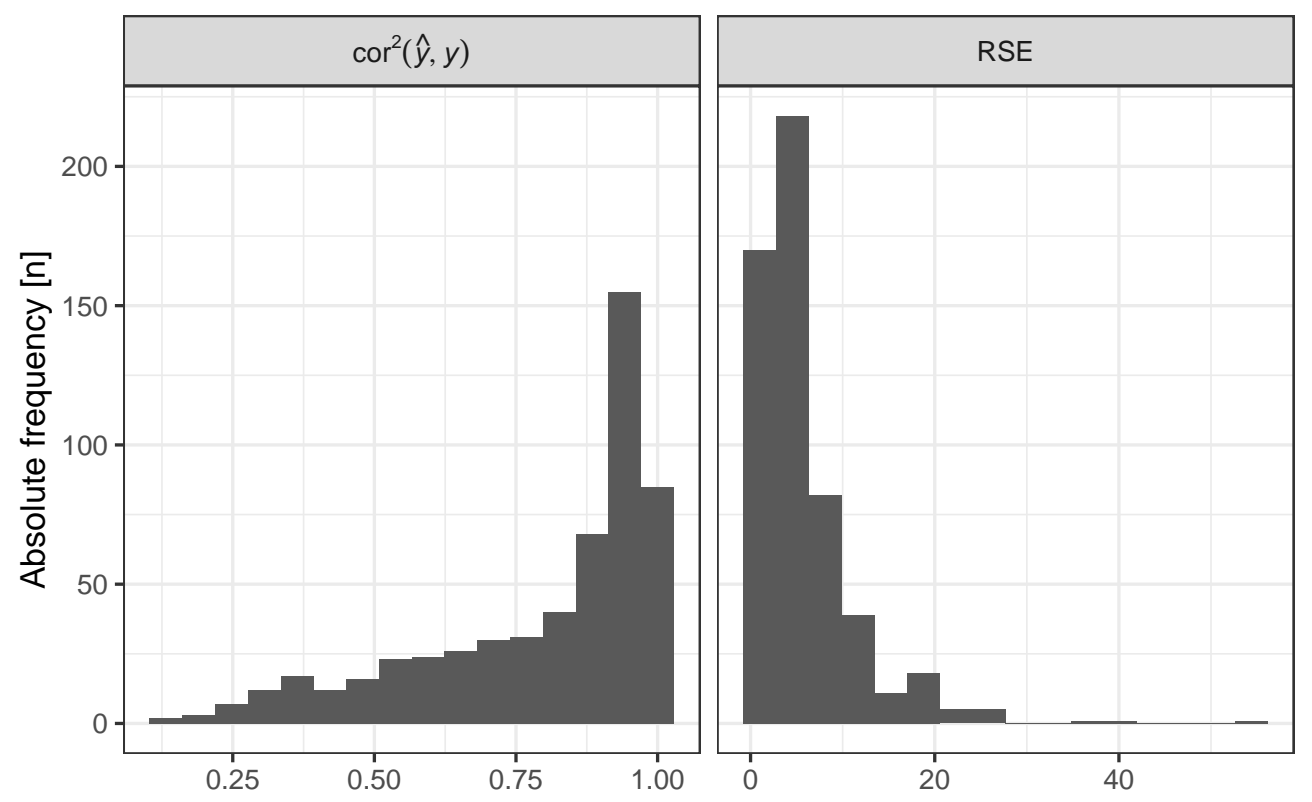

Figure 3.34: Absolute frequency of the squared correlation of observed and fitted values of the adjusted coefficient of determination $\operatorname{cor}^{2}(\hat{y}, y)$ and the residual standard error $\hat{\sigma}$ (RSE) for each linear model fit to single growth unit coordinates in 2D using 2nd-degree polynomials. The differences in scale on the $x$-axis have to be minded here. Included are only the results from fitted models that proved to be significantly better than the null model (see section 3.1.4).

Based on the fitted linear models the differences in curvature angle between internodes were calculated. Since the coordinates were always positioned so that the growth unit base and the tip are at 0 and the highest point of expansion $\left(l_{m}, b\right)$ was positive, the resulting functions always have a negative coefficient for the second term $x^{2}$, which implies a downward opening parabola. The second derivative of a 2 nd-degree polynomial with negative 2 nd coefficient is a negative constant that resembles the rate of change in curvature. Here the differences have been directly calculated in degrees. As the linear modeling necessitated a transformation, the absolute values of the curvature angles were used as a response. The sign is changed back to negative within the structural model.

Figure 3.35 contains the curvature angle differences between succeeding internodes grouped by clone. The values are quite small and the distributions are clearly right skewed for all clones. Differences exist in the form of 'AF2' having the largest angles on average, followed by 'Max 1' and 'Hybride 275' with the 
smallest angles. This points towards more curved GUs for 'AF2' in opposition to 'Hybride 275'.

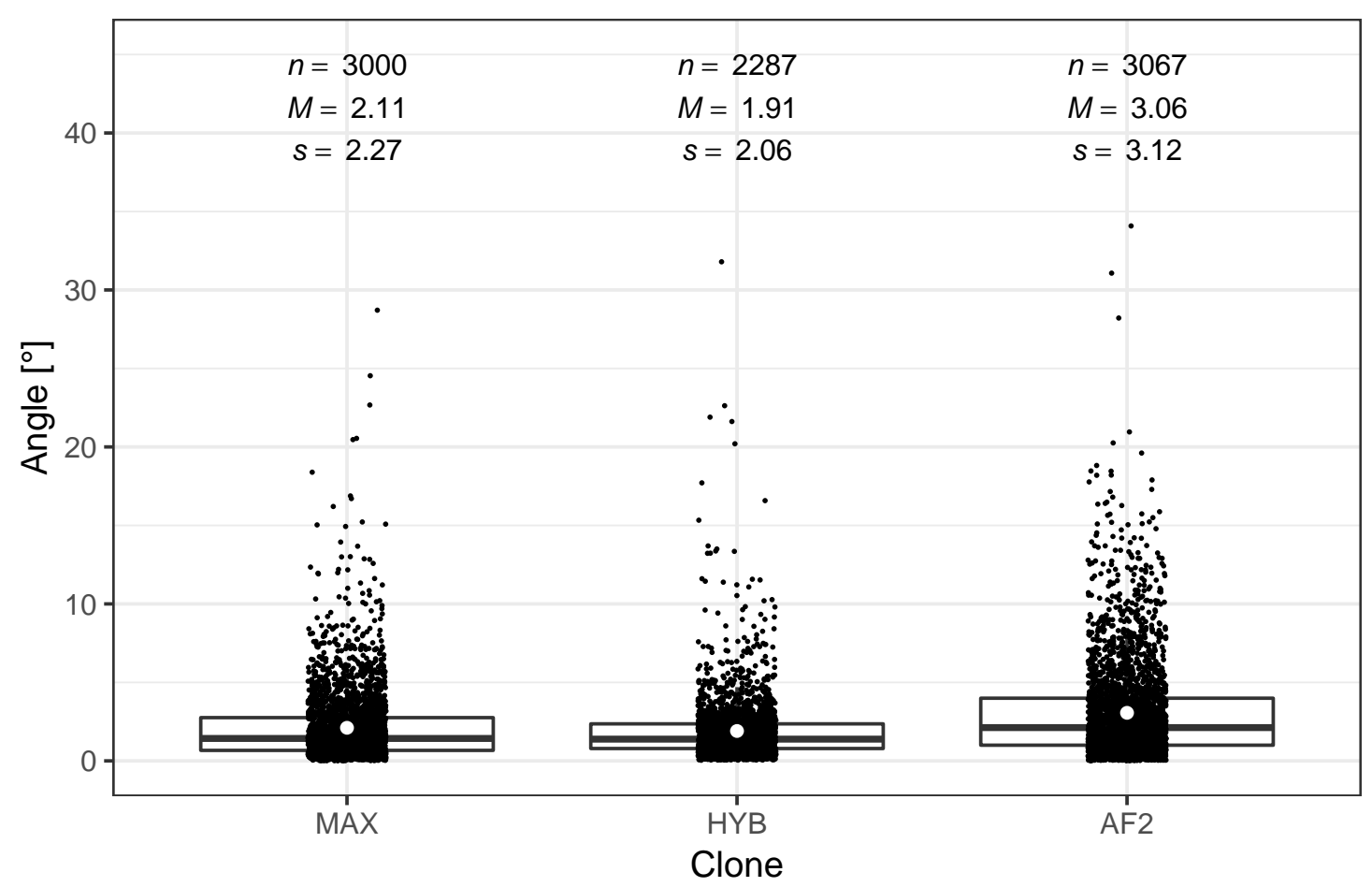

Figure 3.35: Clone-wise comparison of the distribution of curvature angle differences between succeeding internodes. Letters represent groups from pairwise comparisons.

The result of plotting the curvature angles in relation to the GU length can be seen in Figure 3.36. There is a clear decrease in curvature angle with increasing GU length which is trivial in a sense that too large curvature angles for longer growth units would lead to curled shoots. The variance also decreases although some of the increased variance for shorter GUs might be caused due to measurement bias and the bias produced by the data processing (projection, polynomial fitting). 

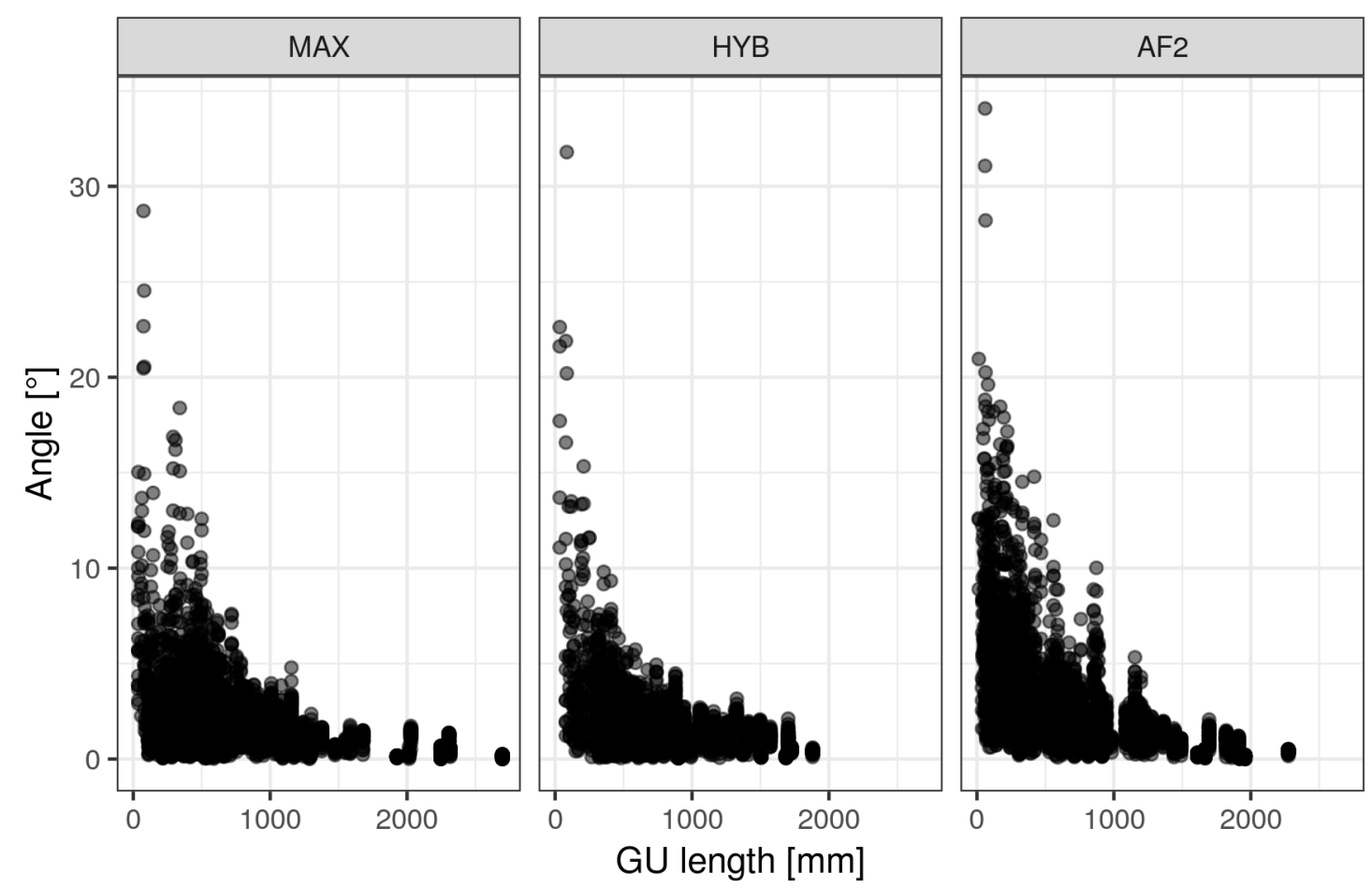

Figure 3.36: Clone-wise comparison of the distribution of curvature angle differences between succeeding internodes. Letters represent groups from pairwise comparisons.

The relationship is obviously nonlinear and could be described by a reciprocal predictor term like $\frac{1}{l}$. The alternative option would be transforming the response which was realized by using the Box-Cox procedure again.

The summaries for the then fitted model with all variables evaluated as sufficient for estimating curvature are shown in Table 3.27. Regarding the predictive power of the model, a value of 0.6 for $R_{a d j}^{2}$ is seen as satisfactory. The main effects for clone and length capture the observations from the two prior graphs well. Furthermore the internode length $i N o d e L$ has a positive influence on curvature. 
Table 3.27: Summary of linear model fit for estimating the curvature angle curvAng. The response has been transformed by raising it to a power of 0.14 . Independent variables are the length of the $\mathrm{GU} l$, the length of the internode $i N o d e L$, the order of the branch order, whether the branch was part of a main or minor stem main, if the GU was a prolongation or lateral shoot proLon and clonal affiliation clone. The colon sign stands for an interaction of two variables. The parameterized model function is given in Equation 3.14.

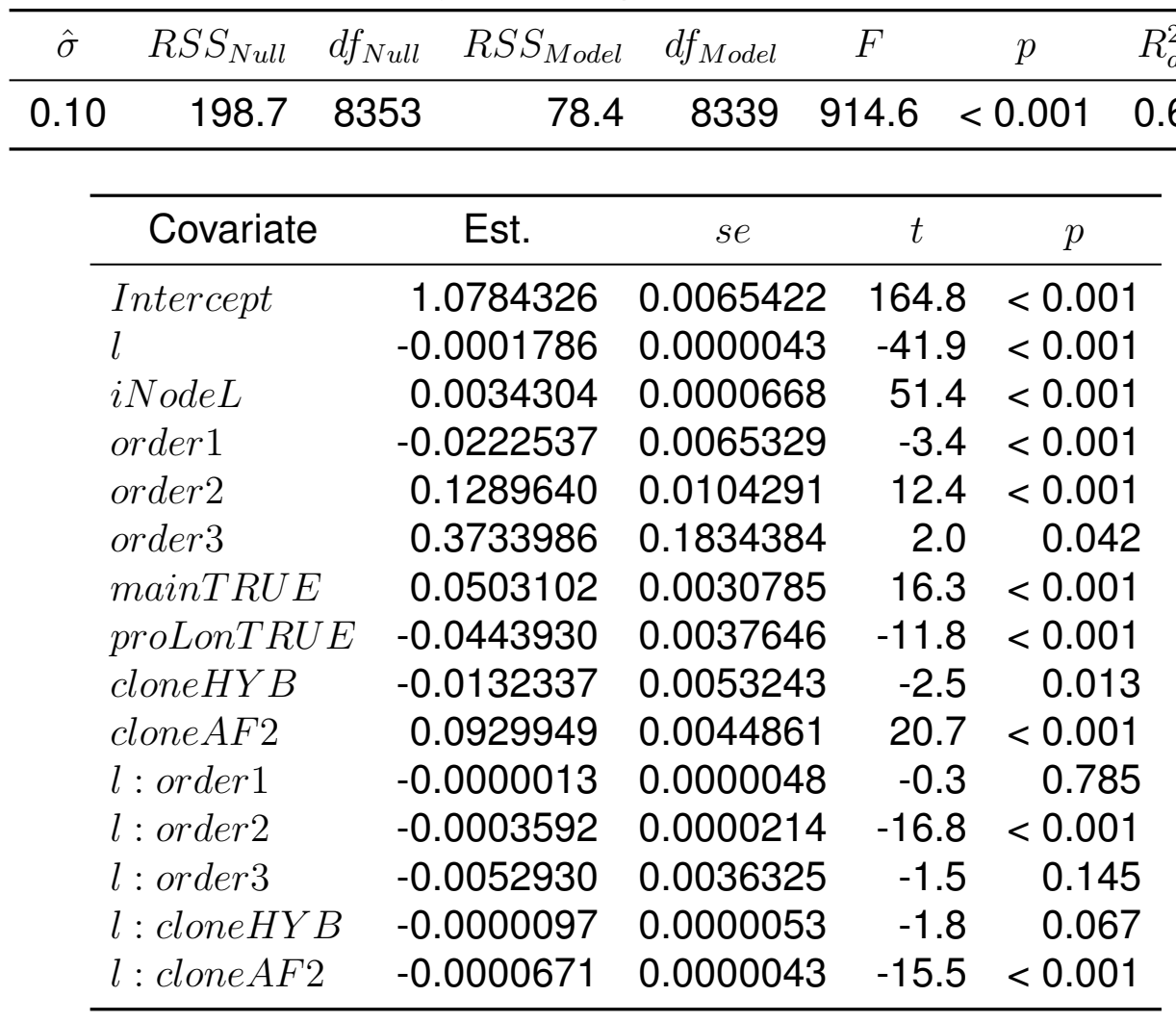


$\widehat{c u r v A n g}^{0.14}=1.0784326$

$$
\begin{aligned}
& +\left(-0.0001786+\left(\begin{array}{c}
0 \\
-0.0000013 \\
-0.0003592 \\
-0.0052930
\end{array}\right) *\right. \text { order } \\
& \left.+\left(\begin{array}{c}
0 \\
-0.0000097 \\
-0.0000671
\end{array}\right) * \text { clone }\right) * l+0.0034304 * \text { iNodeL } \\
& +\left(\begin{array}{c}
0 \\
-0.0222537 \\
0.1289640 \\
0.3733986
\end{array}\right) * \text { order }+0.0503102 * \text { main }-0.0443930 * \text { proLon } \\
& +\left(\begin{array}{c}
0 \\
-0.0132337 \\
0.0929949
\end{array}\right) * \text { clone }
\end{aligned}
$$

The main effects for branch order indicate less curvature for the first and then an increase for the second and third order. It needs to be taken into consideration here that the GUs with order 0 on the main stem are not included here since they were assumed to be straight. The general effect for the main/minor stem distinction is positive for the main stem. The influence of the proLon variable is on the other hand negative indicating that prolongation shoots are bent less. Both interactions of GU length $l$ with order and clone are increasingly reducing the curvature angle in the sequence given in Table 3.27. The coefficients for interaction of $l$ with factor levels first and third branch order as well as clone 'Hybride 275' are not significant.

\subsubsection{Leaf Position and Structure}

Following the steps of the modeling procedure, first, the overall occurrence of leaves within the tree structure was evaluated. The general observation is ob- 
viously that the amount of foliation increases from the ground level to the upper part of the stand and the top of the crown layer. Along the main stem and its side branches towards the top, the probability for nodes bearing leaves increases visibly. This increase correlates with the distribution of 1 year old growth units after the third vegetation period. To model the number of leaves per growth unit a generalized linear model was parameterized. The dependent variable was each GU's relative frequency of nodes bearing leaves to the total number of nodes. Figure 3.37 visualizes the total leaf count per growth unit in relation to its relative height, branch order and clonal affiliation. The relative height is defined here as the relation of the height of the growth unit's mid (relH Mid) to the maximum height of the tree which is usually the tip of the third GU of the main stem (1 year old, order 0 ). The leaf count of 0 does not appear in the displayed data because GUs without any leaves were not measured.

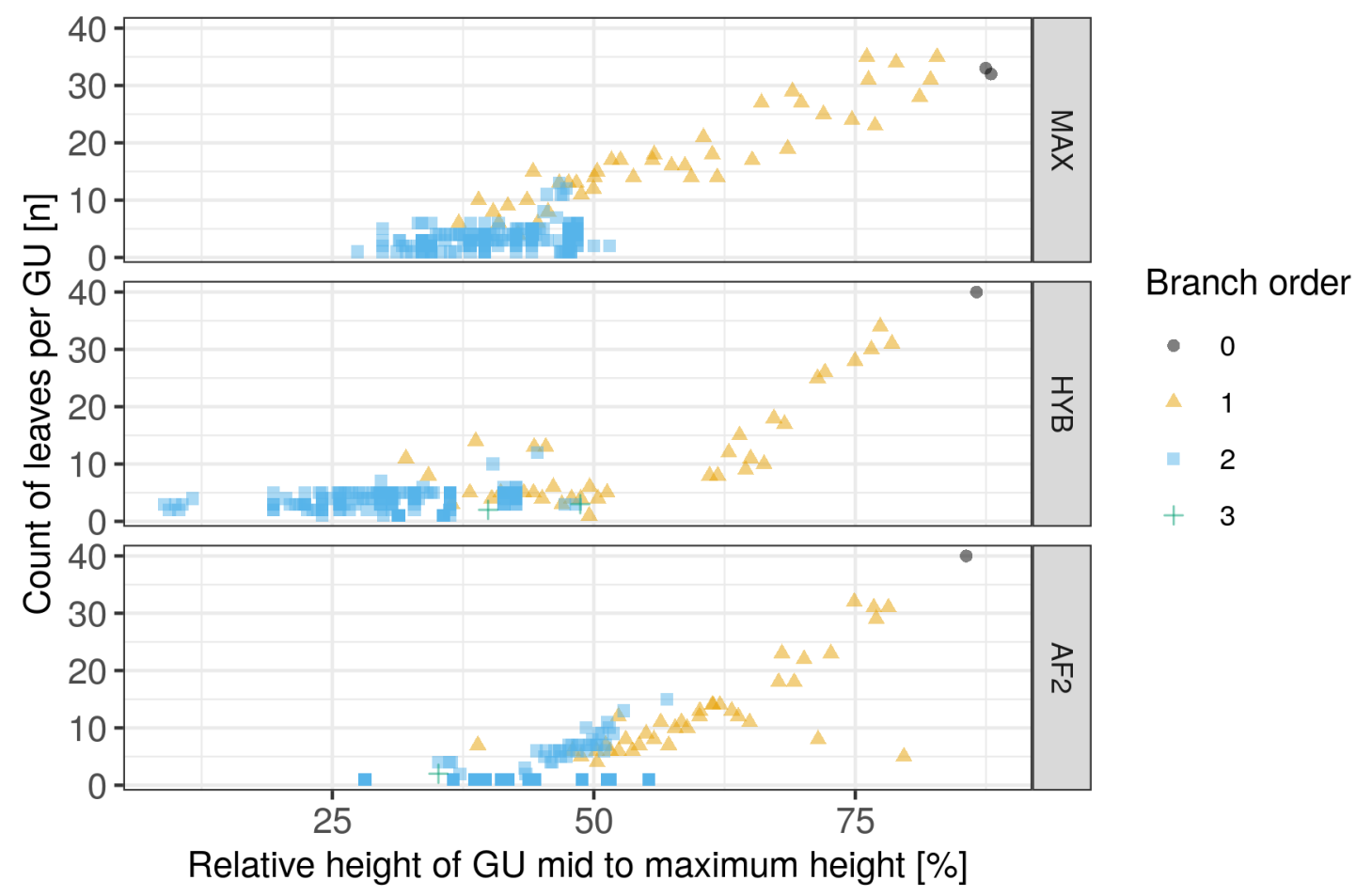

Figure 3.37: Number of leaves per growth unit (GU) by the relative height of the growth unit's mid to the respective tree's maximum height, separated by clone. The different colors and shapes resemble the branch order of each respective growth unit.

The branch order, which can be distinguished by point shape in Figure 3.37 . 
reflects parts of the crown architecture. The shown leaf count is closely correlated with the GU's length. For all clones, a major part of the leaves appear in the tree's lower half on growth units that are comparably short and of the second branch order (blue squares). Towards the tip of the main stem mainly first order shoots carry leaves (yellow triangles). These are longer in comparison to the second order shoots and their length increases with leaf count towards the maximum height. The highest leaves are positioned on the third growth unit of the main stem which is marked by the black dots of branch order 0 . Few third branch order growth units with a small number of leaves were observed in the tree's lower part.

Overall, the relative mid-height of a growth unit seems to be a good predictor of its leaf count. The correlation is linear to slightly nonlinear with minor differences between clones. For modeling the given data, the leaf count could be used directly as a response variable. In this case however, it seemed more sensitive to turn this count into a binary variable nodeLeaf with nodes bearing leaves as a success (1) versus nodes without leaves as a failure (0). This can also be expressed as a proportion per growth unit which can then be used as the response for a generalized linear model in $\mathrm{R}$ with the binomial model family. Figure 3.38 displays this ratio and its correlation with the length of the growth unit. Within the acquired data for 1 year old growth units carrying leaves the growth unit's length and its relative height are closely correlated. 


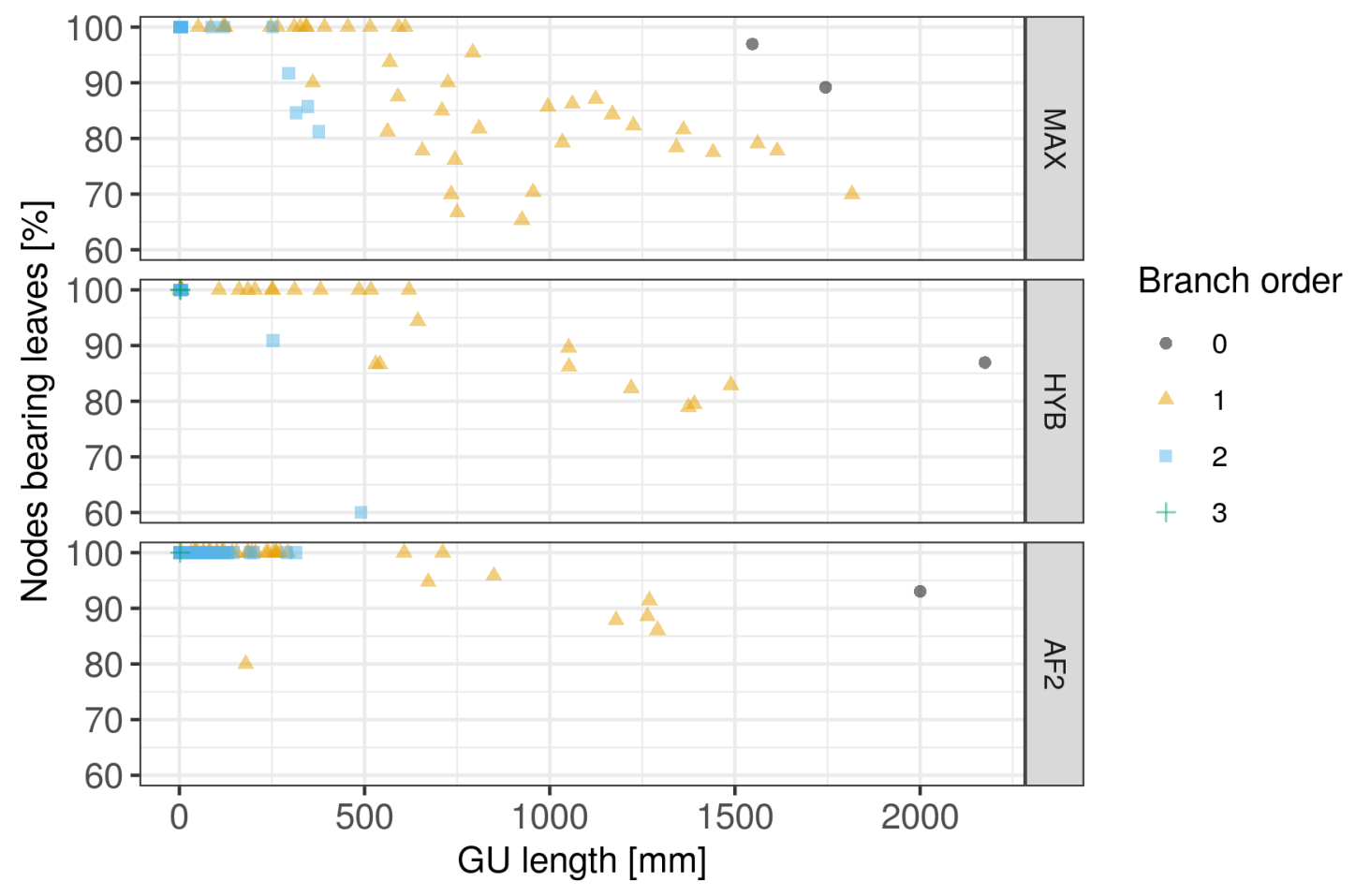

Figure 3.38: Relative frequency of nodes bearing leaves per growth unit (GU) in relation to the growth unit's length, separated by clone. The different colors and shapes resemble the branch order of each respective growth unit.

The diagnostics for the generalized linear model that was parameterized are included in Table 3.28 , 
Table 3.28: Summary of generalized linear model fit for estimating $\eta_{\text {nodeLeaf }}$ of the probability $p_{\text {nodeLeaf }}$ of nodes bearing leaves versus nodes without leaves. Variable $l$ resembles the GU length and clone the clonal affiliation. The colon sign stands for the interaction of two variables. The parameterized model function is given in Equation 3.15 .

\begin{tabular}{|c|c|c|c|c|c|c|c|}
\hline Model (link) & $\Phi$ & $D_{\text {Null }}$ & $d f_{\text {Null }}$ & $D_{\text {Model }}$ & $d f_{\text {Model }}$ & $F$ & $p$ \\
\hline q.-binom. (logit) & 0.27 & 618.7 & 1064 & 148.0 & 1058 & $\begin{array}{l}3 \quad 294.9 \\
\end{array}$ & $9<0.001$ \\
\hline \multicolumn{2}{|c|}{ Covariate } & \multicolumn{2}{|c|}{ Est. } & \multicolumn{2}{|c|}{ se } & $t$ & $p$ \\
\hline \multicolumn{2}{|c|}{ Intercept } & \multicolumn{2}{|c|}{5.0688725} & \multicolumn{2}{|c|}{0.1582719} & 32.0 & $<0.001$ \\
\hline \multirow{2}{*}{\multicolumn{2}{|c|}{$\begin{array}{l}l \\
l^{2}\end{array}$}} & \multicolumn{2}{|c|}{-0.0059519} & \multicolumn{2}{|c|}{0.0002765} & -21.5 & $<0.001$ \\
\hline & & \multicolumn{2}{|c|}{0.0000023} & \multicolumn{2}{|c|}{0.0000001} & 17.7 & $<0.001$ \\
\hline \multicolumn{2}{|c|}{ clone $H Y B$} & \multicolumn{2}{|c|}{0.7597414} & \multicolumn{2}{|c|}{0.2339515} & 3.2 & 0.001 \\
\hline \multicolumn{2}{|c|}{ cloneAF2 } & \multicolumn{2}{|c|}{2.3541775} & \multicolumn{2}{|c|}{0.3665794} & 6.4 & $<0.001$ \\
\hline \multicolumn{2}{|c|}{$l:$ clone $H Y B$} & \multirow{2}{*}{\multicolumn{2}{|c|}{-0.0011157}} & \multirow{2}{*}{\multicolumn{2}{|c|}{$\begin{array}{l}0.0001872 \\
0.0002803\end{array}$}} & -2.7 & 0.008 \\
\hline \multicolumn{2}{|c|}{$l:$ clone $A F 2$} & & & & & -4.0 & $<0.001$ \\
\hline
\end{tabular}

$$
\begin{aligned}
& \widehat{\eta_{\text {nodeLeaf }}}=\log \left(\frac{\widehat{p_{\text {nodeLeaf }}}}{1-p_{\text {nodeLeaf }}}\right)=5.0688725 \\
& +\left(-0.0059519+\left(\begin{array}{c}
0 \\
-0.0004999 \\
-0.0011157
\end{array}\right) * \text { clone }\right) * l \\
& +0.0000023 * l^{2}+\left(\begin{array}{c}
0 \\
0.7597414 \\
2.3541775
\end{array}\right) * \text { clone }
\end{aligned}
$$

The model that was chosen after variable selection is rather simple and relies on the descriptor variables growth unit length $l$ evaluated as first- and seconddegree polynomials, clone and the interaction of clone with $l$. Because of an underdispersion the quasi-binomial approach was chosen hence the dispersion parameter $\Phi<1$. The $D_{a d j}^{2}$ of 0.76 can be seen as good. The estimated coefficients resemble the prior findings quite well. The probability for a node bearing a leaf decreases through the link function with increasing length which is refined 
by the positive second order polynomial term. The coefficients for 'AF2' and 'Hybride 275 ' are significantly higher than for 'Max 1' on the base level although their decrease by length is also higher as indicated by the interaction coefficients.

After now being able to estimate on how many nodes per growth unit leaves are present, the question arises how they are distributed along the growth unit itself. In Figure 3.39 all relative positions of leaf-bearing nodes along the mother growth unit per clone are shown.

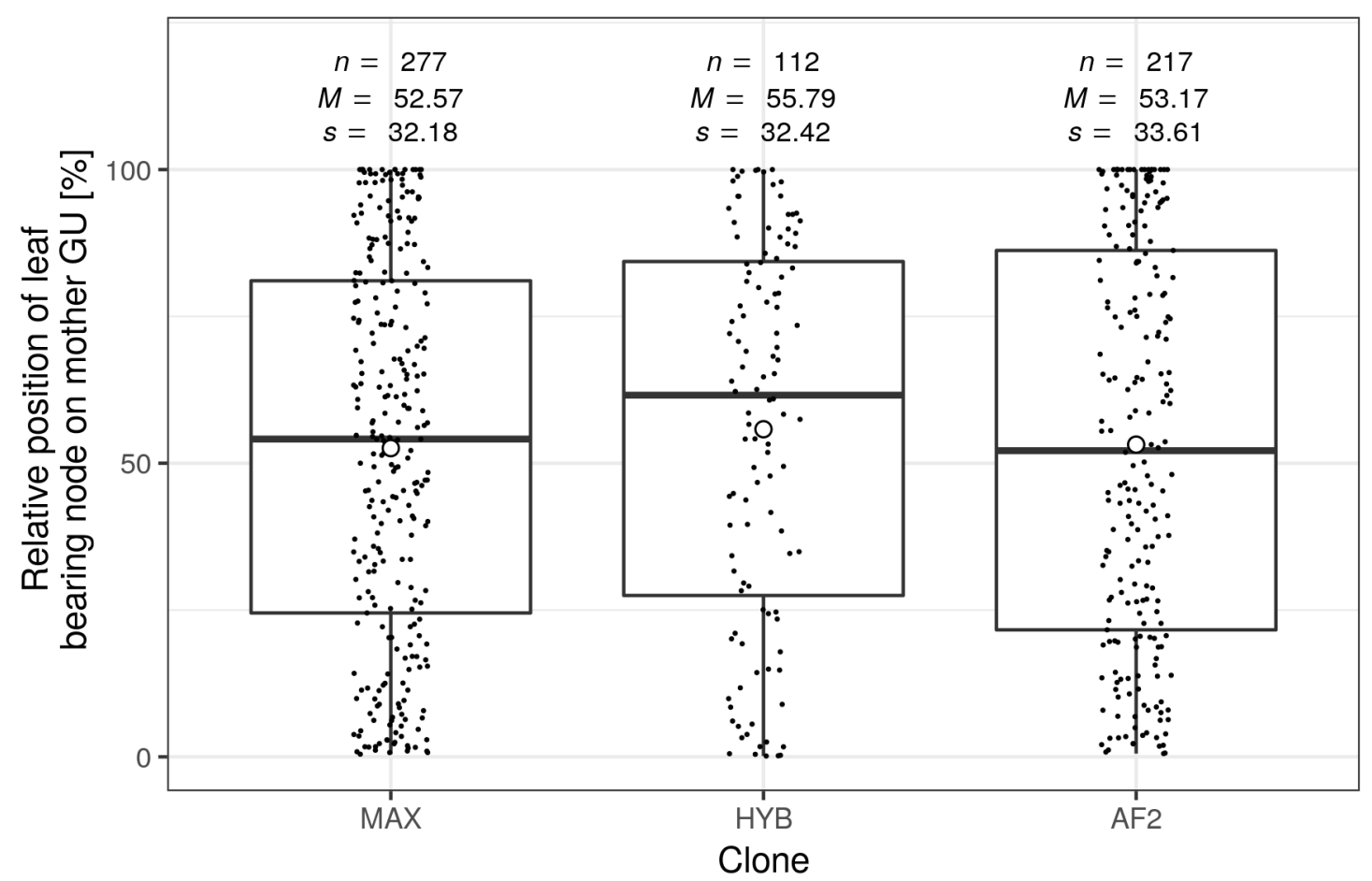

Figure 3.39: Distribution of leaf-bearing nodes by relative position in regard to growth unit length by clone. Leaves on short shoots with a length below $1 \mathrm{~cm}$ are not included.

There is no clear difference in the distribution of values visible. The occurrence of leaves is evenly distributed across the respective mother GU. Variables like clone, age and order were tested for significant influence on the response. None of the tested coefficients differed significantly from 0 , so the values of relative position are assumed to be uniformly random. The accumulation of values at precisely $100 \%$ occurred because the leaves at the growth unit's tip were always measured (see section 3.1.5). 
In Figure 3.40 the increase of leaf size in the form of blade length in relation to relative height becomes visible.

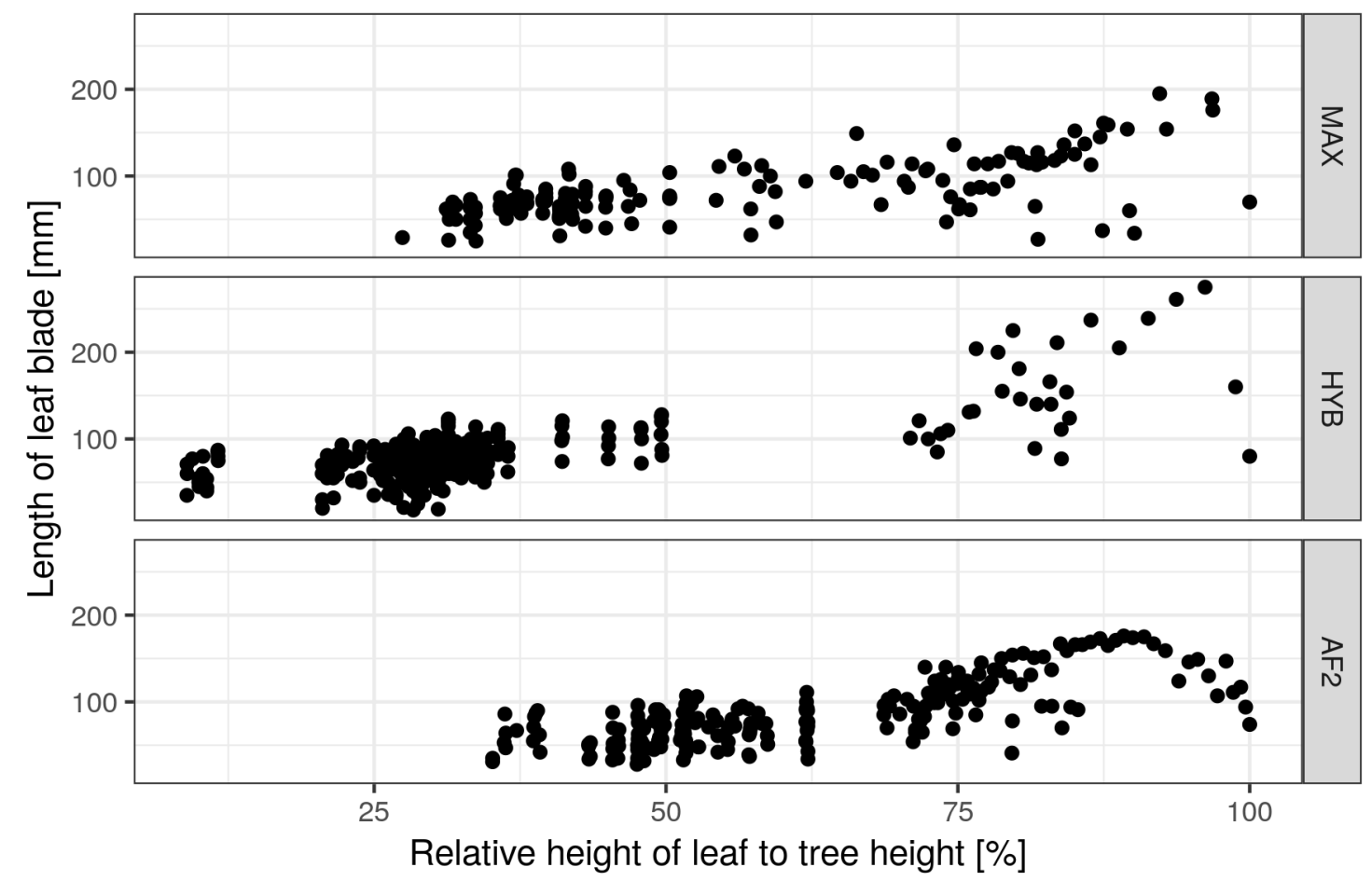

Figure 3.40: Length of leaf blade bladL by the height of the respective leaf in relation to the maximum tree height heightRel, separated by clone.

This tendency exists in all 3 clones, though 'Max 1' and 'AF2' seem to be more similar in size and trend. Leaves from clone 'Hybride 275 ' had higher length values (including the maximum) in the upper crown and some of the leaves that were measured are positioned lower than for the two other clones. While leaf blade length and relative position of the leaf seem to be positively correlated a considerable amount of variation in leaf blade length remains. Especially for 'AF2', there seems to be some curved pattern left within the distribution of value pairs which becomes visible in the right part of the point cloud. This can in part be elucidated with Figure 3.41. Here, the leaf blade length is plotted over the relative position of the leaf in relation to the growth unit's length. Though there is still some noise left, a faint parabolic pattern becomes visible that implies smaller leaves at the growth unit's base with increasing size towards the middle and then decreasing leaf blade length towards the growth unit's tip. The latter might originate from the 
fact that some leaves had not grown to their final size yet since the measurement took place in August.

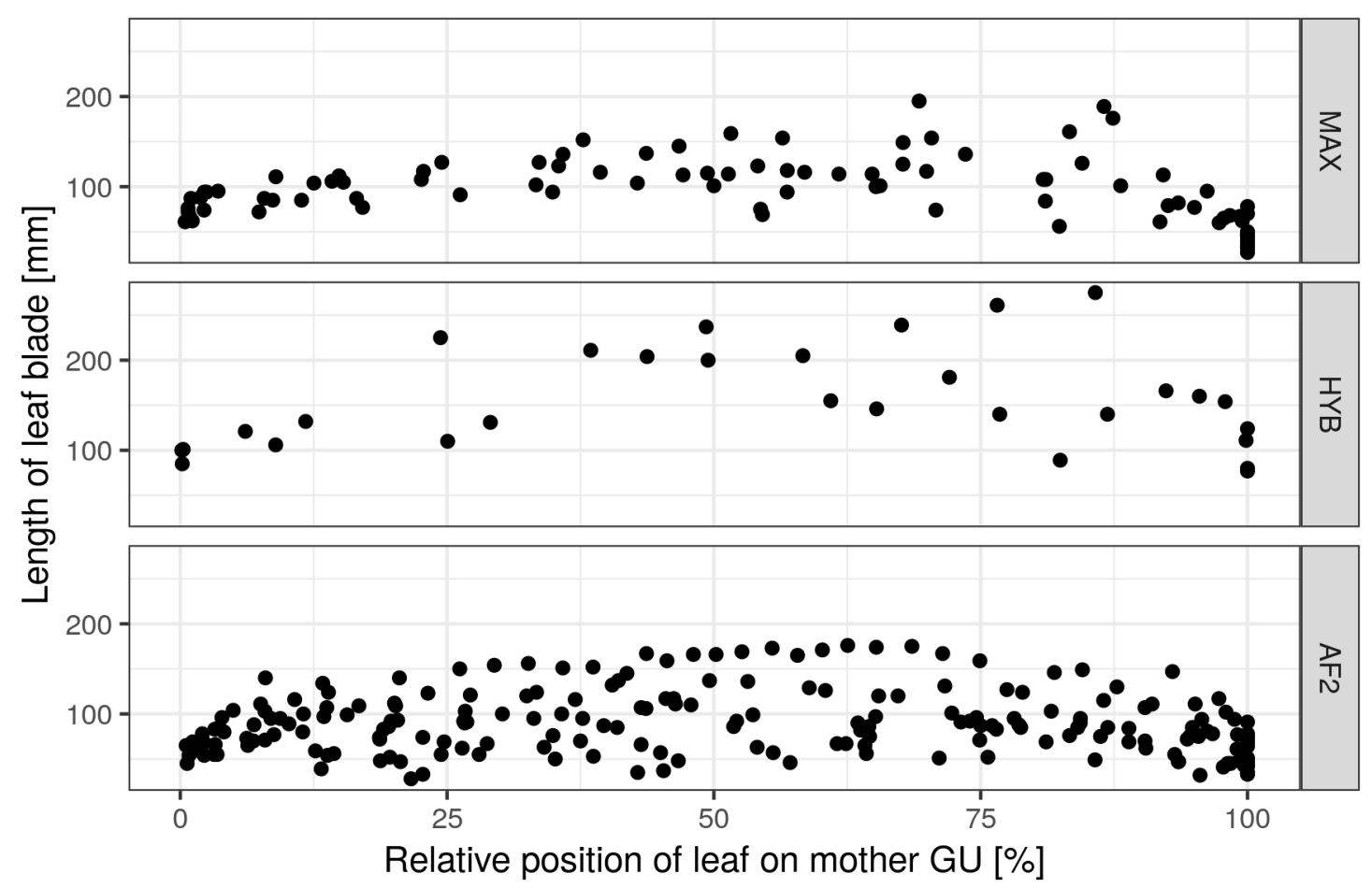

Figure 3.41: Length of leaf blade bladL by the relative position relPos of the respective leaf in relation to the length of its mother growth unit, separated by clone.

For estimating the leaf blade length bladL the two variables of relative height in regard to tree height and relative position on the mother growth unit proved to be sufficient after variable selection. The former covariate was introduced as a linear and square term while the latter was added as a square term as well as their interaction with each other. The factorial covariate clone and its interaction with relative height heightRel also exhibited a significant influence on the response bladL. The model summary data is given in Table 3.29. 
Table 3.29: Summary of linear model fit for estimating the leaf blade length bladL. Independent variables are the height of the respective leaf in relation to the maximum tree height heightRel, the relative position relPos of the respective leaf in relation to the length of its mother GU and clonal affiliation clone. The colon sign stands for an interaction of two variables. The parameterized model function is given in Equation 3.16

\begin{tabular}{ccrrrccc}
\hline$\hat{\sigma}$ & $R S S_{\text {Null }}$ & $d f_{\text {Null }}$ & $R S S_{\text {Model }}$ & $d f_{\text {Model }}$ & $F$ & $p$ & $R_{\text {adj }}^{2}$ \\
\hline 22.96 & 828903.5 & 645 & 335400.4 & 636 & 104.0 & $<0.001$ & 0.59 \\
\hline
\end{tabular}

\begin{tabular}{lrrrr}
\hline \multicolumn{1}{c}{ Covariate } & \multicolumn{1}{c}{ Est. } & \multicolumn{1}{c}{$s e$} & \multicolumn{1}{c}{$t$} & \multicolumn{1}{c}{$p$} \\
\hline Intercept & 99.63 & 13.55 & 7.4 & $<0.001$ \\
heightRel & -228.77 & 50.64 & -4.5 & $<0.001$ \\
heightRel $^{2}$ & 335.42 & 44.75 & 7.5 & $<0.001$ \\
relPos $^{2}$ & -36.74 & 15.07 & -2.4 & 0.015 \\
cloneHYB $_{\text {cloneAF2 }}$ & -13.50 & 6.97 & -1.9 & 0.053 \\
heightRel $:$ cloneHYB & -28.98 & 8.56 & -3.4 & $<0.001$ \\
heightRel : cloneAF2 $^{2}$ & 43.35 & 12.93 & 6.2 & $<0.001$ \\
heightRel $:$ relPos $^{2}$ & 231.90 & 13.62 & 3.2 & 0.001 \\
heightRel $^{2}:$ relPos $^{2}$ & -306.98 & 57.26 & -5.4 & $<0.001$ \\
\hline
\end{tabular}

$$
\begin{aligned}
\widehat{\text { bladL }}= & 99.63+\left(-228.77+\left(\begin{array}{c}
0 \\
80.35 \\
43.89
\end{array}\right) * \text { clone }\right) * \text { heightRel }+335.42 * \text { heightRel }^{2} \\
& -36.74 * \text { relPos }^{2}+\left(\begin{array}{c}
0 \\
-13.50 \\
-28.98
\end{array}\right) * \text { clone }+231.90 * \text { heightRel } * \text { relPos }^{2} \\
& -306.89 * \text { height Rel }^{2} * \text { relPos }^{2}
\end{aligned}
$$

The predictive power of the model is satisfactory with an $R_{a d j}^{2}=0.59$. The estimated effects for variables heightRel, heightRel ${ }^{2}$ and relPos $^{2}$ capture the impressions from the last two graphs. The intercept is reduced for clones 'Hybride 275' and 'AF2' although only for the latter the effect is significant. On the contrary, the 
influence of heightRel is significantly increased for these two clones in comparison to 'Max 1'.

A graph of the relationship between leaf blade length and width is given in Figure 3.42. There is a definite positive correlation between both variables, but the correlation is not strictly linear. In comparison, clones 'AF2' and 'Max 1' have shorter and broader leaves than 'Hybride 275'. For clone 'Max 1' the broadest leaves were observed. 'Hybride 275 ' has contrasting properties with longer, slender leaves.

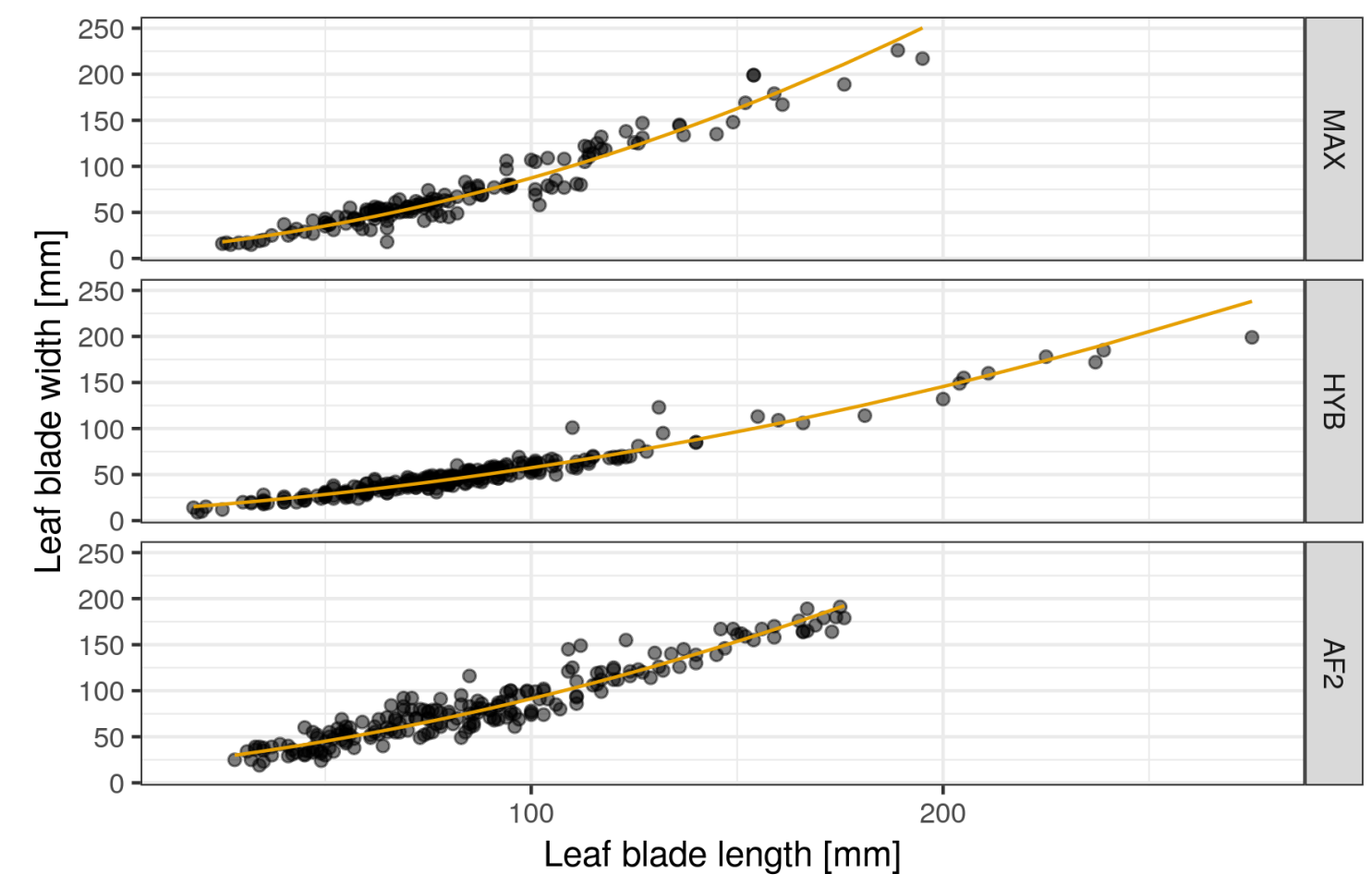

Figure 3.42: Relationship between leaf lamina width and leaf lamina length per clone. Solid yellow lines are curves for back-transformed estimations from the linear model (see Table 3.30).

For modeling the relationship, the response was square root transformed. The independent variables that showed a significant influence on leaf width bladW (see Table 3.30) and hence were chosen for the model, were clone, length of leaf blade bladL and their interaction clone : bladL. 
Table 3.30: Summary of linear model fit for estimating the leaf blade width bladW. The dependent variable has been square root transformed. Independent variables are the leaf blade length $b l a d L$ and clonal affiliation clone. The colon sign stands for an interaction of two variables. The parameterized model function is given in Equation 3.17 .

\begin{tabular}{|c|c|c|c|c|c|c|c|c|}
\hline$\hat{\sigma}$ & $R S S_{\text {Null }}$ & $d f_{\text {Null }}$ & $R S S_{\text {Model }}$ & $d f_{\text {Model }}$ & $F$ & & $p$ & $R_{a d j}^{2}$ \\
\hline 0.57 & 3014.9 & 624 & 201.0 & 619 & 1733. & C & .001 & 0.93 \\
\hline \multicolumn{3}{|c|}{ Covariate } & Est. & se & & $t$ & \multicolumn{2}{|l|}{$p$} \\
\hline \multicolumn{3}{|c|}{ Intercept } & 2.53453 & 0.1233 & & 20.5 & \multicolumn{2}{|c|}{$<0.001$} \\
\hline \multicolumn{3}{|c|}{ bladL } & 0.06814 & 0.0014 & & 48.7 & \multicolumn{2}{|c|}{0.001} \\
\hline \multirow{2}{*}{\multicolumn{3}{|c|}{$\begin{array}{l}\text { clone H Y B } \\
\text { clone AF2 }\end{array}$}} & 0.55650 & 0.1523 & & 3.7 & \multicolumn{2}{|c|}{$<0.001$} \\
\hline & & & 1.35222 & 0.1607 & & 8.4 & \multicolumn{2}{|c|}{$<0.001$} \\
\hline \multicolumn{3}{|c|}{ bladL : clone $H Y B$} & -0.02326 & 0.0017 & & 13.6 & $<0.0$ & \\
\hline \multicolumn{3}{|c|}{ bladL: clone $A F 2$} & -0.01144 & 0.0017 & & -6.5 & \multicolumn{2}{|c|}{$<0.001$} \\
\hline
\end{tabular}

$$
\begin{aligned}
\widehat{\sqrt{\text { bladW }}=} & 2.53453+\left(0.06814+\left(\begin{array}{c}
0 \\
-0.02326 \\
-0.01144
\end{array}\right) * \text { clone }\right) * \text { bladL } \\
& +\left(\begin{array}{c}
0 \\
0.55650 \\
1.35222
\end{array}\right) * \text { clone }
\end{aligned}
$$

By $R_{a d j}^{2}=0.93$, the predictive power of the model is as expected very high. This model has rather informal character since the leaf shape is further evaluated in section 3.2 .10 which includes the leaf blade length and width correlation.

Figure 3.43 displays the value pairs of leaf-wise petiole (stalk) length and leaf blade length. A positive correlation can be seen for all 3 clones. The correlation of both variables in the sample could be modeled via a linear model with a seconddegree polynomial function for leaf blade length bladL and clone as a factorial independent variable. The effect for interaction of the leaf blade length, its square and clone could not be assumed to be different from 0 . Hence the common slope and only differing intercept in all 3 panels in Figure 3.43. 


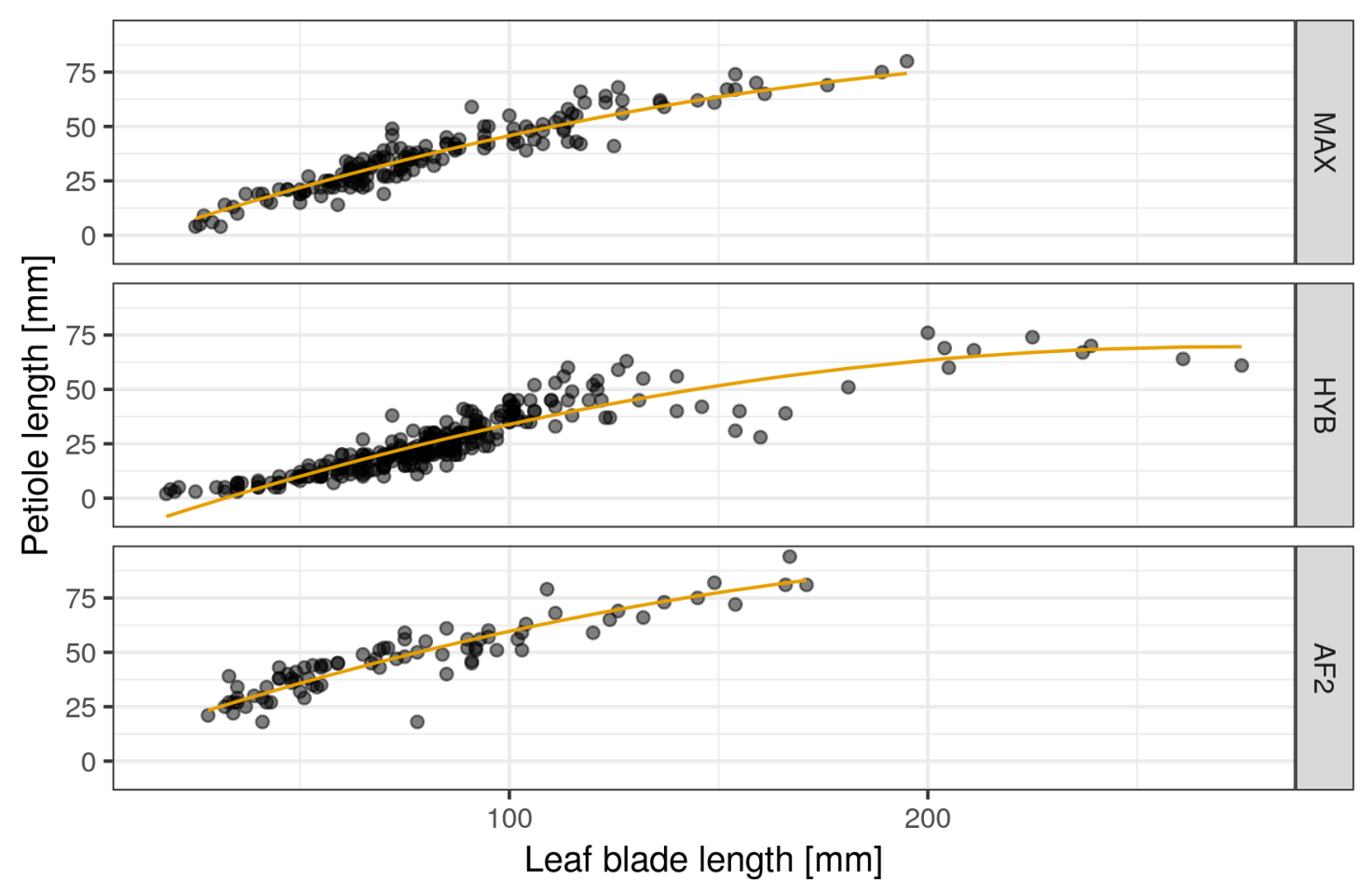

Figure 3.43: Relationship between leaf petiole length and leaf blade length per clone. Solid yellow lines represent estimations from the linear model (see Table 3.31.

The summary data for the linear model is included in Table 3.31 . 
Table 3.31: Summary of linear model fit for estimating the leaf petiole length petiL. Independent variables are the leaf blade length bladL and clonal affiliation clone.

\begin{tabular}{|c|c|c|c|c|c|c|}
\hline$\hat{\sigma}$ & $R S S_{\text {Null }}$ & $d f_{\text {Null }}$ & $R S S_{\text {Model }}$ & $l \quad d f_{\text {Model }}$ & $F$ & $p$ \\
\hline 6.02 & 153440.7 & 506 & 18217.7 & 502 & 931.5 & $<0.001$ \\
\hline & Covariate & & Est. & se & $t$ & $p$ \\
\hline & Intercept & & .08295 & 1.34441 & -6.0 & $<0.001$ \\
\hline & bladL & & .66021 & 0.02435 & 27.1 & $<0.001$ \\
\hline & blad $L^{2}$ & & .00122 & 0.00010 & -11.7 & $<0.001$ \\
\hline & clone $H Y B$ & & .84817 & 0.60712 & -19.5 & $<0.001$ \\
\hline & clone $A F 2$ & & .88658 & 0.83053 & 16.7 & $<0.001$ \\
\hline
\end{tabular}

With an $R_{a d j}^{2}$ of approximately 0.88 the predictive power of the model is comparatively high. The longest petioles were observed for clone 'AF2'. On average, the ranking for petiole length is 'AF2' followed by 'Max 1' and 'Hybride 275' with the shortest mean petiole length. A caveat should be added that for 'Hybride 275' the model predicts negative petiole length values for very small leaf blade lengths. Below a leaf blade length of $50 \mathrm{~mm}$ the mean value for petiole length is $5.7 \mathrm{~mm}$ with a standard deviation of $2.16 \mathrm{~mm}$.

Assessing the correlation of diameter and length of leaf petiole in Figure 3.44 . as expected, longer leaf petioles imply thicker petioles overall. There are differences between the clones though. While 'AF2' develops thinner petioles with increased length, 'Hybride 275' forms thicker leaf petioles with comparable length. Clone 'Max 1' takes up an intermediate role. 


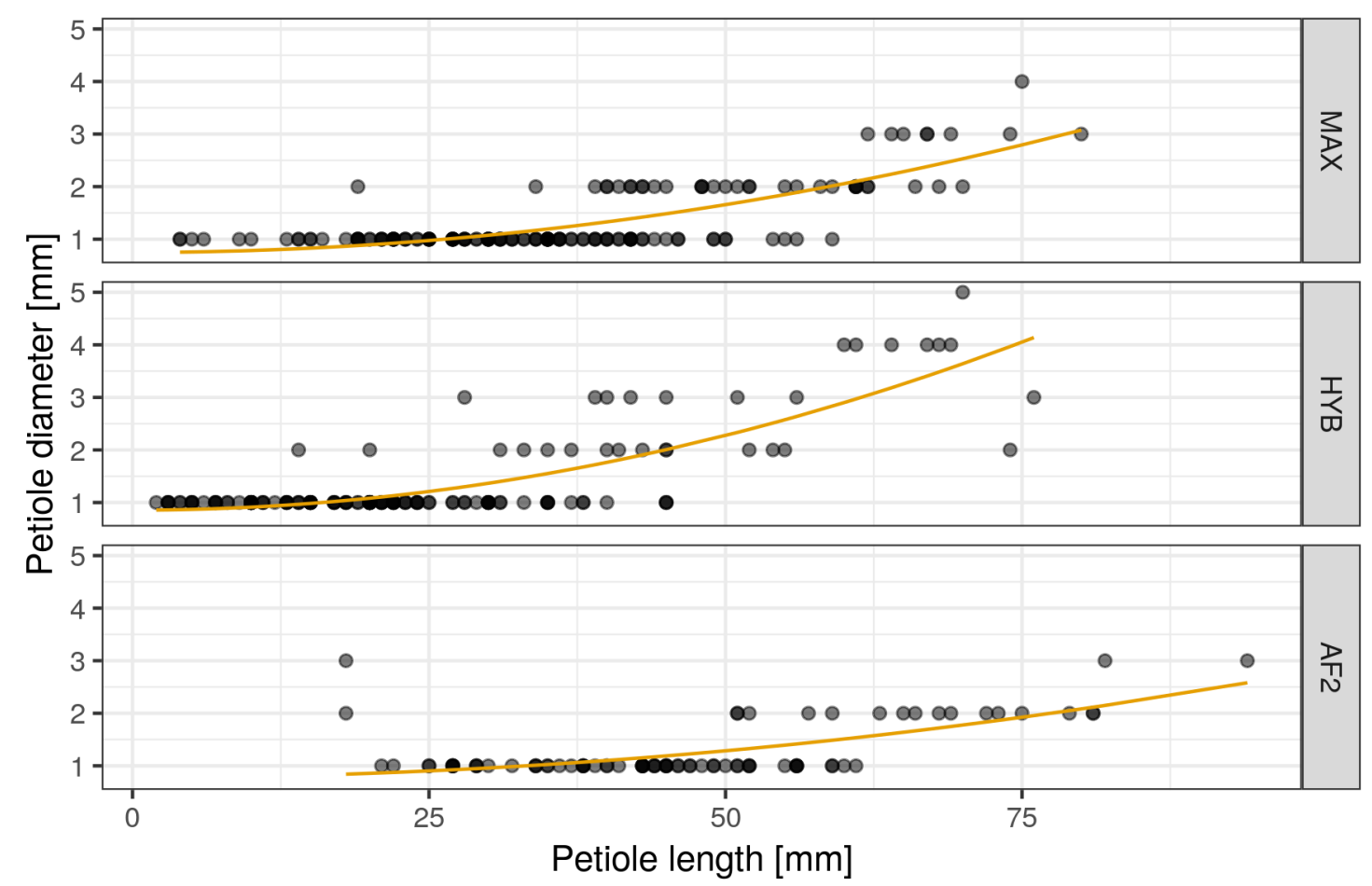

Figure 3.44: Relationship between leaf petiole diameter and length per clone. Solid yellow lines are curves for back-transformed estimations from the linear model (see Table 3.32).

The functions that are displayed in Figure 3.44 are based on the squared petiole length peti $L$, the factor clone and their interaction as independent variables (see Table 3.32). The predictive power of the model is satisfactory with a value of 0.62 for $R_{a d j}^{2}$. 
Table 3.32: Summary of linear model fit for estimating the leaf petiole diameter petiD. Independent variables are the leaf petiole length peti $L$ and clonal affiliation clone. The parameterized model function is given in Equation 3.18 .

\begin{tabular}{|c|c|c|c|c|c|c|c|c|}
\hline$\hat{\sigma}$ & $R S S_{\text {Null }}$ & $d f_{\text {Null }}$ & $R S S_{\text {Model }}$ & $d f_{\text {Model }}$ & $F$ & \multicolumn{2}{|r|}{$p$} & $R_{a d j}^{2}$ \\
\hline 0.41 & 178.3 & 392 & 66.6 & 387 & 129. & \multicolumn{2}{|c|}{0.001} & 0.62 \\
\hline \multicolumn{3}{|c|}{ Covariate } & Est. & \multicolumn{2}{|l|}{ se } & $t$ & \multicolumn{2}{|c|}{$p$} \\
\hline \multirow{2}{*}{\multicolumn{3}{|c|}{$\begin{array}{l}\text { Intercept } \\
\text { pet } i L^{2}\end{array}$}} & 0.74806 & 0.052 & & 14.3 & \multicolumn{2}{|c|}{$<0.001$} \\
\hline & & & 0.00036 & 0.000 & & 14.4 & $<0$. & 001 \\
\hline \multicolumn{3}{|c|}{ clone $H Y B$} & 0.10662 & 0.067 & & 1.6 & \multicolumn{2}{|c|}{$\begin{array}{r}<0.001 \\
0.113\end{array}$} \\
\hline \multicolumn{3}{|c|}{ clone $A F 2$} & 0.02722 & 0.096 & & 0.3 & \multicolumn{2}{|c|}{0.778} \\
\hline \multirow{2}{*}{\multicolumn{3}{|c|}{ petiL $L^{2}$ : clone $H Y B$}} & 0.00020 & 0.000 & & 5.3 & \multicolumn{2}{|c|}{$<0.001$} \\
\hline & & & -0.00016 & 0.000 & & -4.3 & \multicolumn{2}{|c|}{$<0.001$} \\
\hline
\end{tabular}

$\widehat{\text { petiD }}=0.74806+\left(0.00036+\left(\begin{array}{c}0 \\ 0.00020 \\ -0.00016\end{array}\right) *\right.$ clone $) *$ peti $L^{2}+\left(\begin{array}{c}0 \\ 0.10662 \\ 0.02722\end{array}\right) *$ clone

For the orientation angle petiW between internode and petiole and for the angle between leaf blade and petiole petiBladW, no significant correlation with other variables was found that could sufficiently explain the angles on a morphological basis. Variables of leaf structure, orientation and morphology were tested as explanatory variables but did not yield satisfactory results. The decision was made to take the mean model with factor clone for data generation in GrolMP (see Figures 3.45 and 3.46 . 


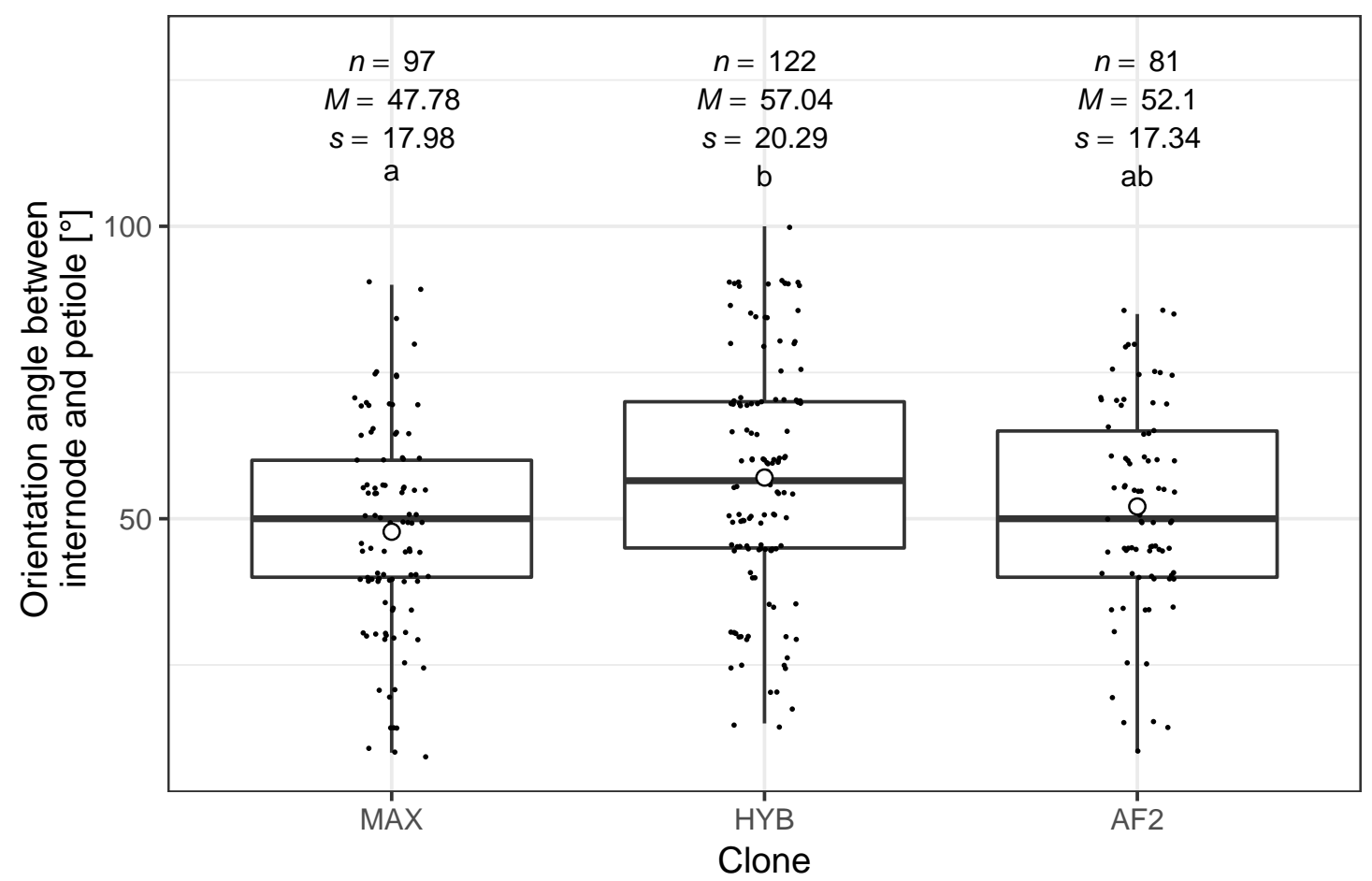

Figure 3.45: Comparison of distribution of orientation angle between internode and petiole petiW by clone. Letters represent groups from pairwise comparisons.

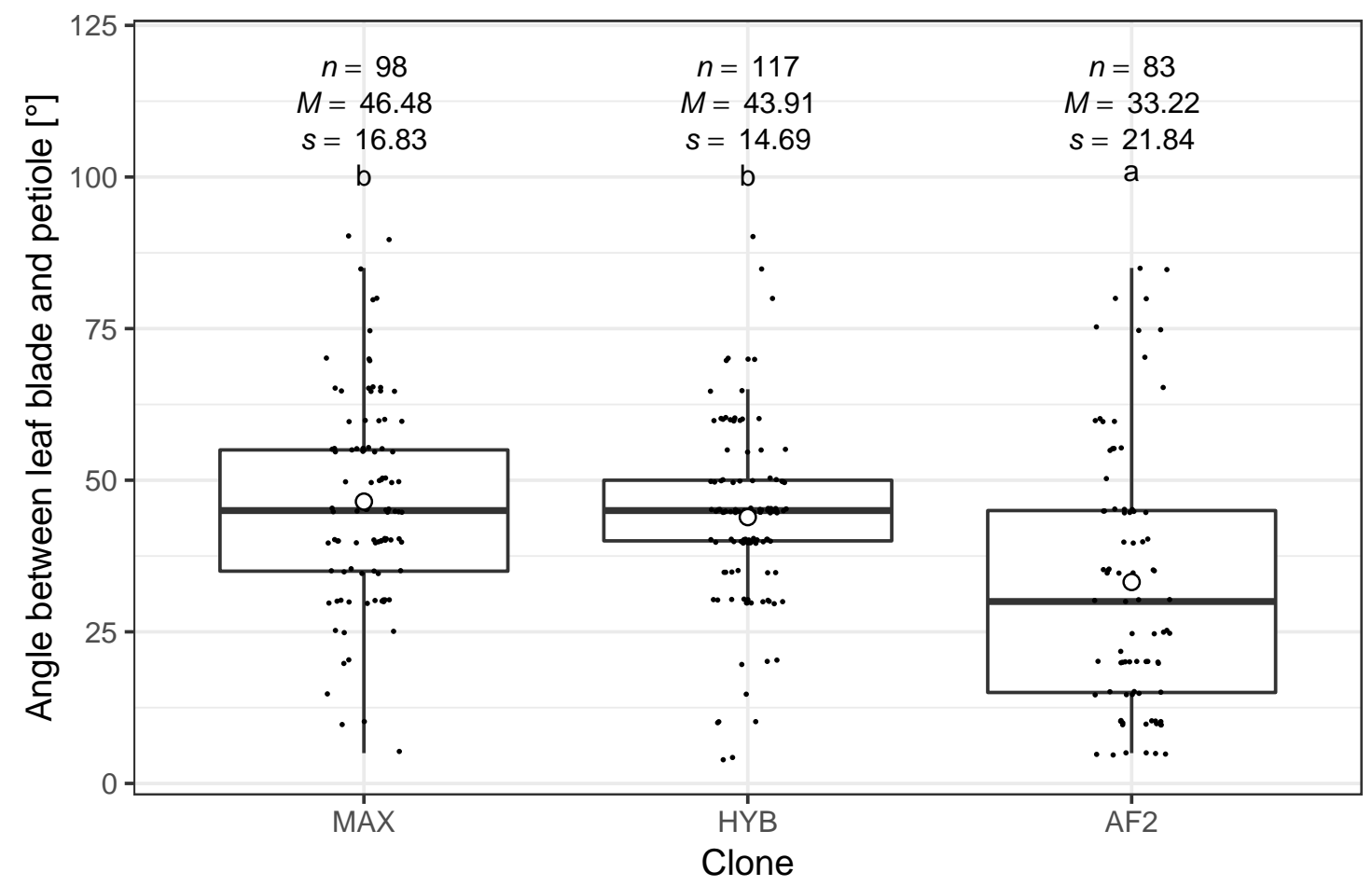

Figure 3.46: Comparison of distribution of angle between leaf blade and petiole petiBladW by clone. Letters represent groups from pairwise comparisons. 
Clone 'Hybride 275' has the most obtuse mean angle for peti $W$ with $57.04^{\circ}$. With average $52.1^{\circ}$, 'AF2' angles are intermediate and 'Max 1' has more acute angles with a mean of $47.78^{\circ}$. After pairwise comparisons, significant mean differences could only be found between 'Hybride 275' and 'Max 1'. Comparable values were measured for the angle petiBladW for 'Max 1' and 'Hybride 275' with an average of $46.48^{\circ}$ and $43.91^{\circ}$ respectively. The measured angles for 'AF2' differed significantly from the two other clones with $33.22^{\circ}$. The differences in mean angles for peti $W$ and petiBladW seem comparably low between clones while the clone wise and overall variances are rather high. The clonal mean models only explain 4 respectively $8 \%$ of the variance (see Tables 3.33 and 3.34).

Table 3.33: Summary of linear model fit for estimating the angle between internode and petiole peti $W$. Independent variable is the clonal affiliation clone.

\begin{tabular}{cccccccc}
\hline$\hat{\sigma}$ & $R S S_{\text {Null }}$ & $d f_{\text {Null }}$ & $R S S_{\text {Model }}$ & $d f_{\text {Model }}$ & $F$ & $p$ \\
\hline 18.79 & 109567.3 & 299 & 104894.5 & 297 & 6.6 & 0.002 \\
\hline \multicolumn{1}{c}{ Covariate } & Est. & se & \multicolumn{1}{c}{$t$} & $p$ \\
\cline { 2 - 7 } & Intercept & 47.78 & 1.91 & 25.0 & $<0.001$ \\
& cloneHYB & 9.26 & 2.56 & 3.6 & $<0.001$ \\
& cloneAF2 & 4.32 & 2.83 & 1.5 & 0.128 \\
\hline
\end{tabular}

Table 3.34: Summary of linear model fit for estimating the angle between leaf blade and and petiole petiBladW. Independent variable is the clonal affiliation clone.

\begin{tabular}{cccccccc}
\hline$\hat{\sigma}$ & $R S S_{\text {Null }}$ & $d f_{\text {Null }}$ & $R S S_{\text {Model }}$ & $d f_{\text {Model }}$ & $F$ & $p$ \\
\hline 17.63 & 100423.4 & 297 & 91639.7 & 295 & 14.1 & $<0.00$ \\
\hline & & & & & & \\
\cline { 2 - 7 } & Covariate & \multicolumn{1}{c}{ Est. } & se & $t$ & $p$ \\
\cline { 2 - 7 } & Intercept & 46.48 & 1.78 & 26.1 & $<0.001$ \\
cloneHYB & -2.57 & 2.41 & -1.1 & 0.289 \\
& cloneAF2 & -13.26 & 2.63 & -5.0 & $<0.001$ \\
\hline
\end{tabular}

The last leaf orientation parameter that was measured is the rotational angle of the leaf blade petiBladR. Figure 3.47 shows the distribution of this variable's 
values by clone and relative leaf height. Along the tree's height, the values are somewhat evenly distributed for clones with balsam poplar parents. The values range between horizontally oriented leaves $\left(90^{\circ}\right)$ to slightly vertically tilted blades $\left(45^{\circ}-225^{\circ}\right.$ and $\left.135^{\circ}-315^{\circ}\right)$. The distribution stays balanced from the lower to the upper part of the tree. Clone 'AF2' however exhibits a different pattern where the frequency shifts in a way that mainly vertically aligned leaves are present in the upper part of the crown. The petiBladR angles are categorized as interval data. It was opted for a linear model here using the transformed interval midpoints as values of the response variable. The curve resulting from the back-transformed estimated values from the parameterized model are displayed in Figure 3.47 .

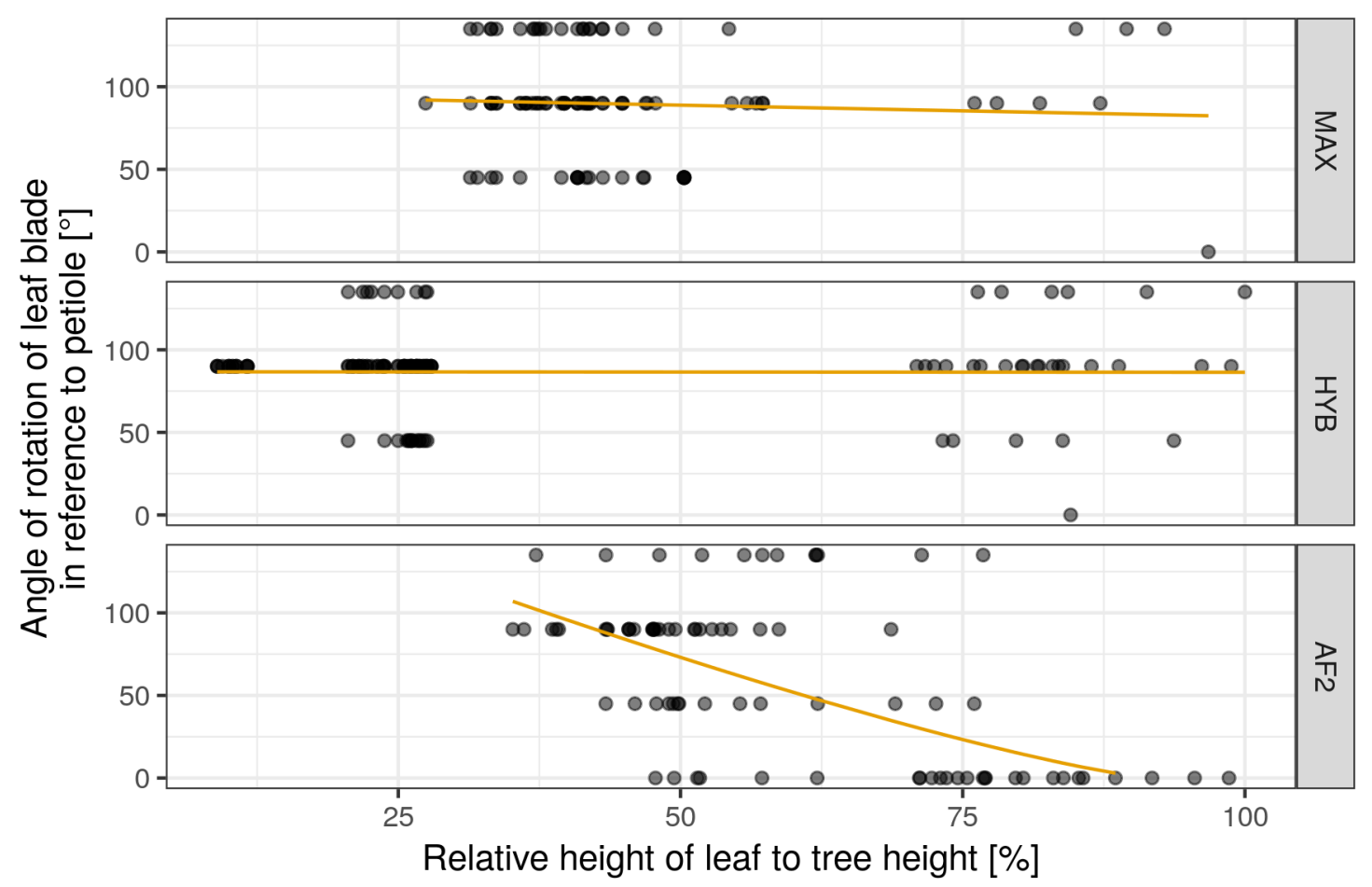

Figure 3.47: Estimation of leaf petiole diameter by petiole length per clone. Solid yellow lines represent back-transformed estimations from linear models (see Table 3.35).

While the variable selection indicated to keep variables clone, heightRel and their interaction, which makes sense based on the already made observations, the $t$-test in Table 3.35 points towards the fact that the coeffcient of heightRel is not significantly different from 0 . The large standard errors for all coefficients should be minded here which in part are based on the fact that rotation angles 
and their classification are used here. It was decided to keep the model as it is displayed here as it has some practicable value for estimating the rotation. Still, the overall goodness of fit is comparably low with a value of 0.32 for $R_{a d j}^{2}$ (see Table 3.35).

Table 3.35: Summary of linear model fit for estimating the angle of rotation of the leaf blade in reference to petiole petiBladR. The response was transformed as petiBlad $R^{0.8}$. Independent variables are the height of the respective leaf in relation to the maximum tree height heightRel and the clonal affiliation clone. The parameterized model function is given in Equation 3.19.

\begin{tabular}{|c|c|c|c|c|c|c|}
\hline$\hat{\sigma}$ & $R S S_{\text {Null }}$ & $d f_{\text {Null }} \quad R S_{i}$ & Model & $d f_{\text {Model }}$ & $F$ & $p$ \\
\hline 11.37 & 56633.3 & 299 & 979.1 & 294 & 28.9 & $<0.001$ \\
\hline \multicolumn{3}{|c|}{ Covariate } & Est. & se & $t$ & $p$ \\
\hline \multicolumn{3}{|c|}{ Intercept } & 38.46 & 3.87 & 9.9 & $<0.001$ \\
\hline \multicolumn{3}{|c|}{ heightRel } & -4.50 & 8.27 & -0.5 & 0.587 \\
\hline \multicolumn{3}{|c|}{ clone $H Y B$} & -2.96 & 4.26 & -0.7 & 0.488 \\
\hline \multicolumn{3}{|c|}{ clone $A F 2$} & 29.65 & 6.35 & 4.7 & $<0.001$ \\
\hline \multicolumn{3}{|c|}{ heightRel : clone $H Y B$} & 4.40 & 9.14 & 0.5 & 0.63 \\
\hline \multicolumn{3}{|c|}{ heightRel : cloneAF2 } & -69.79 & 911.68 & -6.0 & $<0.001$ \\
\hline
\end{tabular}

$$
\begin{aligned}
\text { peti } \widehat{\text { Blad }} R^{0.8}= & 38.46+\left(-4.50+\left(\begin{array}{c}
0 \\
4.40 \\
-69.79
\end{array}\right) * \text { clone }\right) * \text { heightRel } \\
& +\left(\begin{array}{c}
0 \\
-2.96 \\
29.65
\end{array}\right) * \text { clone }
\end{aligned}
$$

\section{Leaves in short shoots}

In section 3.1.5 it was established that multiple short shoots with a growth unit length below $10 \mathrm{~mm}$ were observed on all 3 clones. The quantitative differences between clones are presented in Table 3.36 . 
Table 3.36: Contingency table for frequencies of growth units with length $l<$ $10 \mathrm{~mm}$ separated by clone and branch order. Further counts are given grouped by whether a single (leaf $N=1$ ) or multiple (leaf $N>1$ ) leaves per growth unit had developed. The count of sylleptic shoots within the latter is given in the last column.

\begin{tabular}{lr|r|rrr}
\hline Clone & Branch order & Total [n] & leaf $N=1[\mathrm{n}]$ & leaf $N>1[\mathrm{n}]$ & Sylleptic [n] \\
\hline MAX & 2 & 230 & 33 & 197 & 5 \\
HYB & 1 & 15 & 1 & 14 & 0 \\
HYB & 2 & 198 & 21 & 177 & 2 \\
HYB & 3 & 2 & 0 & 2 & 2 \\
AF2 & 1 & 1 & 0 & 1 & 1 \\
AF2 & 2 & 470 & 468 & 2 & 0 \\
AF2 & 3 & 1 & 0 & 1 & 1 \\
\hline
\end{tabular}

The majority of these short shoots occur as second branch order shoots. While the clones 'Max 1' and 'Hybride 275' show a high percentage of whorl-like short shoots with a count of leaves higher than 1 (ca. $85.7 \%$ and $89.4 \%$ respectively), 'AF2' has a reversed partitioning with $99.6 \%$ single leaved short shoots. Therefore whorl-like short shoots occur much more often for 'Hybride 275' and 'Max 1'. As can be seen in Figure 3.37, shoots shorter than $10 \mathrm{~mm}$, which were observed to have a leaf count of up to 6 leaves, are mainly positioned below $50 \%$ of the tree's relative height. For 'Max 1' and 'AF2' these short shoots can be found between 25 and $50 \%$, for 'Hybride 275' they were also observed further below. If any of these whorl-like short shoots were observed with 1 year old growth units as their mother, these must be sylleptic shoots. The overall quantity for all clones is comparably low (see Table 3.36: 5 growth units for 'Max 1', 4 for 'Hybride 275' and 2 for 'AF2'). Possible differences in leaf morphology by association to short shoots have already been scrutinized by incorporating the length or the relative height of the mother growth unit as a possible independent variable into variable selection for the models described in this section. Most prominently, this has played a direct role in modeling leaf blade length (see Table 3.29). Since leaf blade length is a good proxy for leaf size and morphology, an influence of shoot length on blade length is also extended to further variables in the modeling procedure described in this section. Another variable that is influenced by leaf position and length of mother growth unit is the directional angle of petiole to shoot, petiR. 
It was already stated that the leaf arrangement is alternate based on the structural data. With the leaf morphology data, it became evident that there was a change in the angle distribution of petiR in shorter shoots below $10 \mathrm{~mm}$ length. Figure 3.48 shows the GU-wise mean difference of directional angles petiR between consecutive leaves along a growth unit. For this analysis, the peti $R$ values were grouped by whorl-like short shoot and sorted in ascending order and the difference in angle between consecutive leaves was calculated.

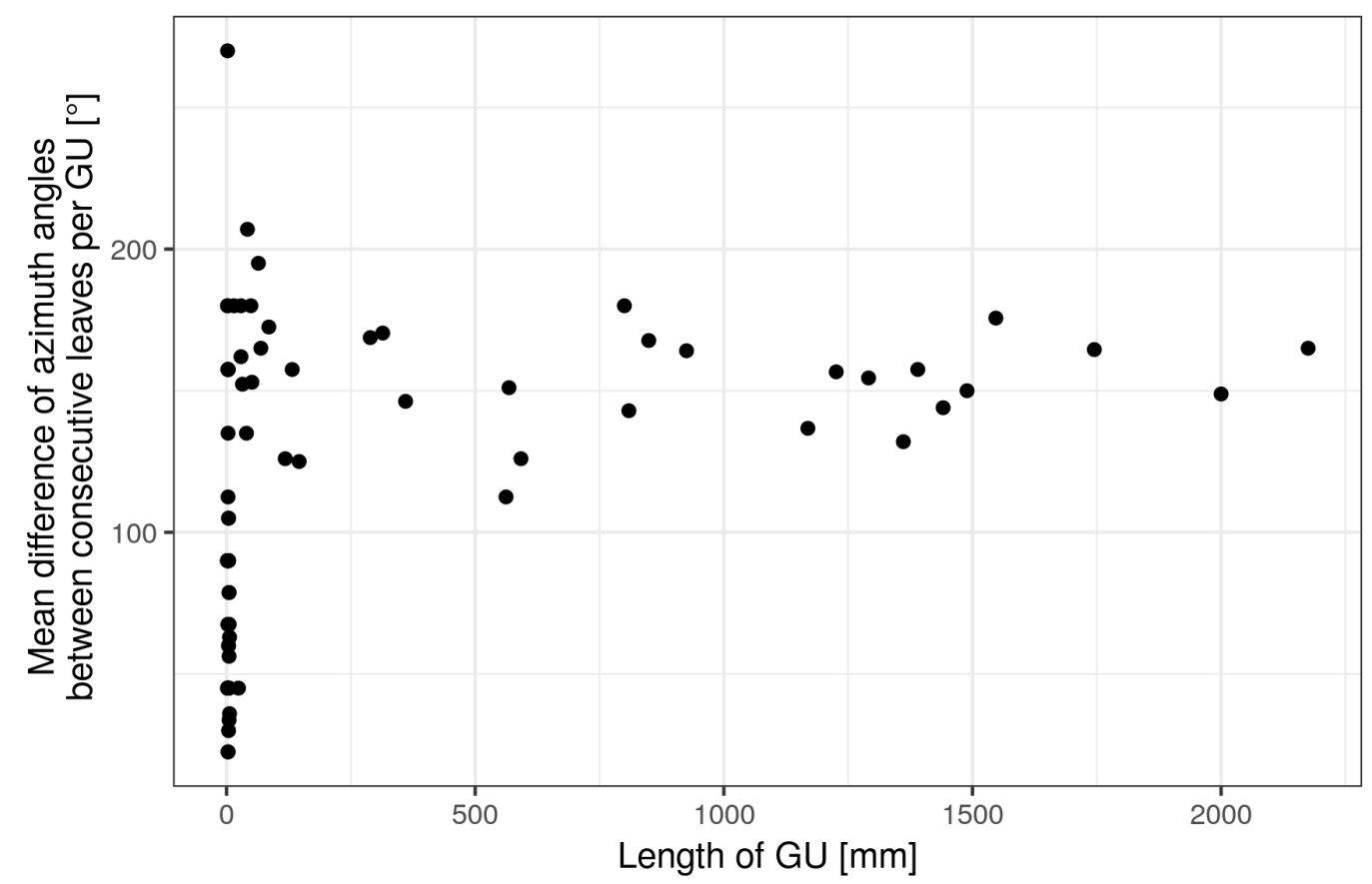

Figure 3.48: Mean differences of leaves' directional angles petiR between consecutive leaves by length of the respective growth unit.

For a wide range of growth unit's lengths (> $10 \mathrm{~mm}$ to $2175 \mathrm{~mm}$ ) the mean value is $154^{\circ}$ with a standard deviation of $27.6^{\circ}$. This points towards said alternate leaf arrangement and confirms the findings from the structural data. Despite that, for very short growth units a much higher variance can be observed. When focusing on growth units smaller than $10 \mathrm{~mm}$ a different trend is revealed for single value differences in directional angle between consecutive leaves (see Figure 3.49). 


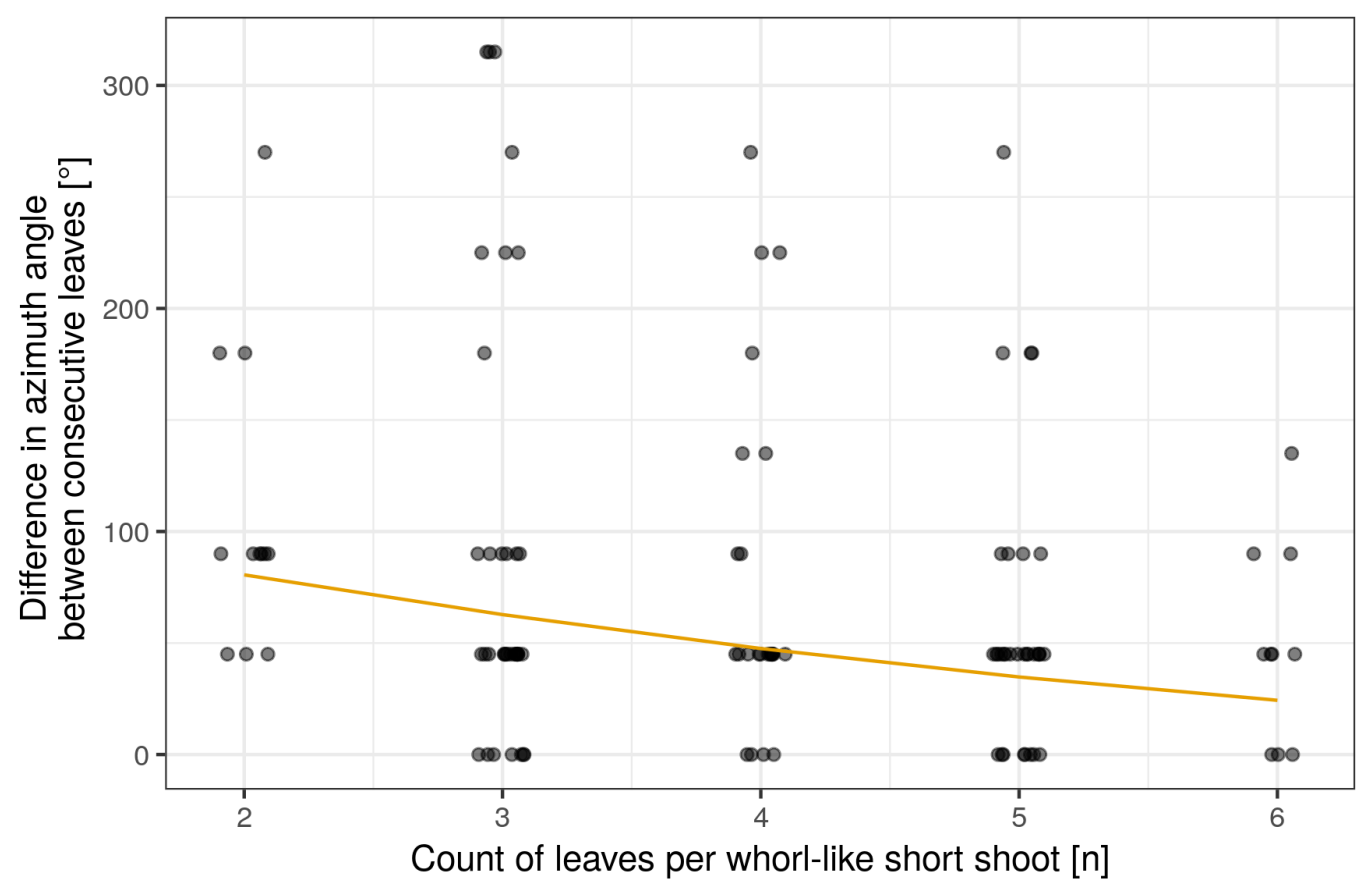

Figure 3.49: Differences in directional angle petiR between consecutive leaves by count of leaves of the respective growth unit. Shown here is only data from whorl-like short shoots (GU length $<1 \mathrm{~cm}$ and count of leaves $>1$ ). A slight jitter along the $x$-axis was added to the data points for better distinction of quantity.

The difference in angle reduces with increasing number of leaves for whorllike short shoots with 2 to 6 leaves. This points towards a more evenly circular distribution of leaves when the leaf count is higher per whorl-like short shoot. The overall model fit is comparably poor (see Table 3.37) with an $R_{a d j}^{2}$ of 0.05 .

Table 3.37: Summary of linear model fit for estimating the differences in directional angle petiR between consecutive leaves in whorl like short shoots (GU length $<1 \mathrm{~cm}$ and count of leaves $>1$ ). Independent variable is the number of leaves per whorl-like short shoot.

\begin{tabular}{crrrrrrr}
\hline$\hat{\sigma}$ & $R S S_{\text {Null }}$ & $d f_{\text {Null }}$ & $R S S_{\text {Model }}$ & $d f_{\text {Model }}$ & $F$ & $p$ \\
\hline 2.71 & 868.6 & 113 & 821.5 & 112 & 6.4 & 0.0 \\
\hline & & \multicolumn{7}{c}{} & & \\
\cline { 2 - 7 } & Covariate & Est. & se & $t$ & $p$ \\
\cline { 2 - 7 } & Intercept & 6.89 & 0.89 & 7.7 & $<0.001$ \\
& leaf $N$ & -0.55 & 0.22 & -2.5 & 0.013 \\
\hline
\end{tabular}




\subsubsection{Leaf Shape}

Figure 3.50 contains the shapes of all leaves after digitizing with the image processing tool by Henke et al. (2014). The pairs of successive $x$ - and $y$-values have been connected for each leaf. Based on this graph differences in shape and size become apparent between the clones and the sections that the leaves were sampled within. Also the change of shape with size can be observed here.

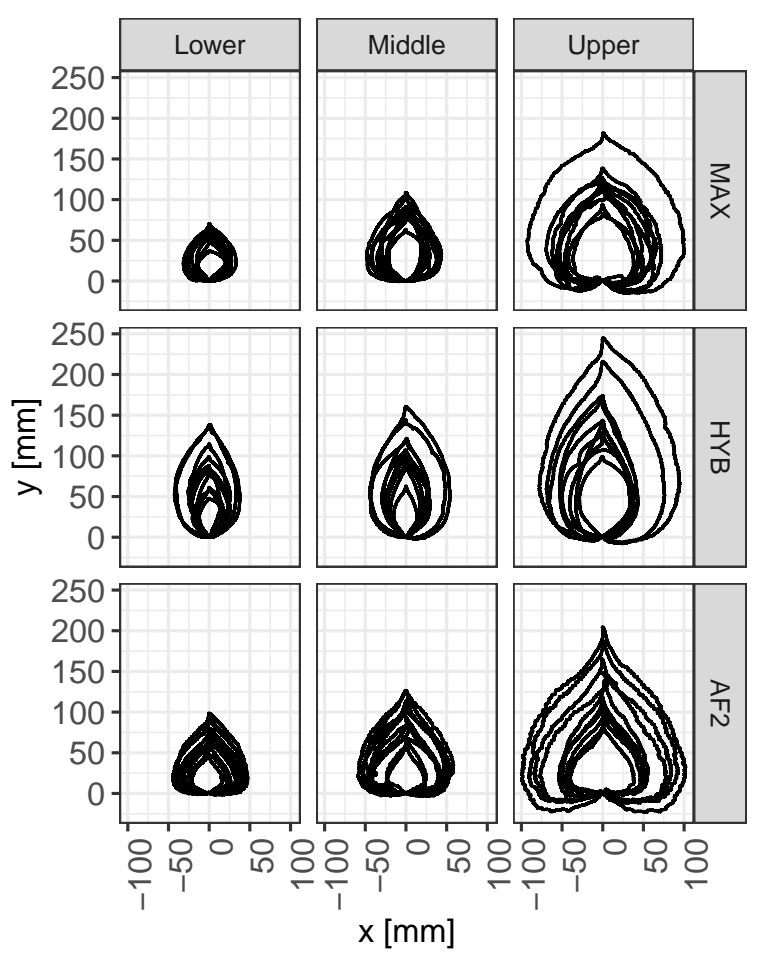

Figure 3.50: Leaf shapes after digitizing with the image processing tool by Henke et al. (2014).

Using the classification by Hickey (1973) (Figure 3.51) the leaf shapes can be described as follows: The shape of the lamina (leaf blade) for all clones is predominantly symmetric. For clone 'Max 1' the leaf form shifts from elliptic to ovate for smaller to bigger leaves since the height of the greatest width moves closer to the leaf base with increasing length. The leaf apex is mucronate to acuminate with the apex culminating in a sharp point at the end of the midvein. The leaf base for smaller leaves is obtuse to rounded, becomes truncate and then cordate with increasing size. Clone 'Hybride 275' exhibits a more elliptic leaf form for smaller and medium sized leaves since the vertical position of the greatest 


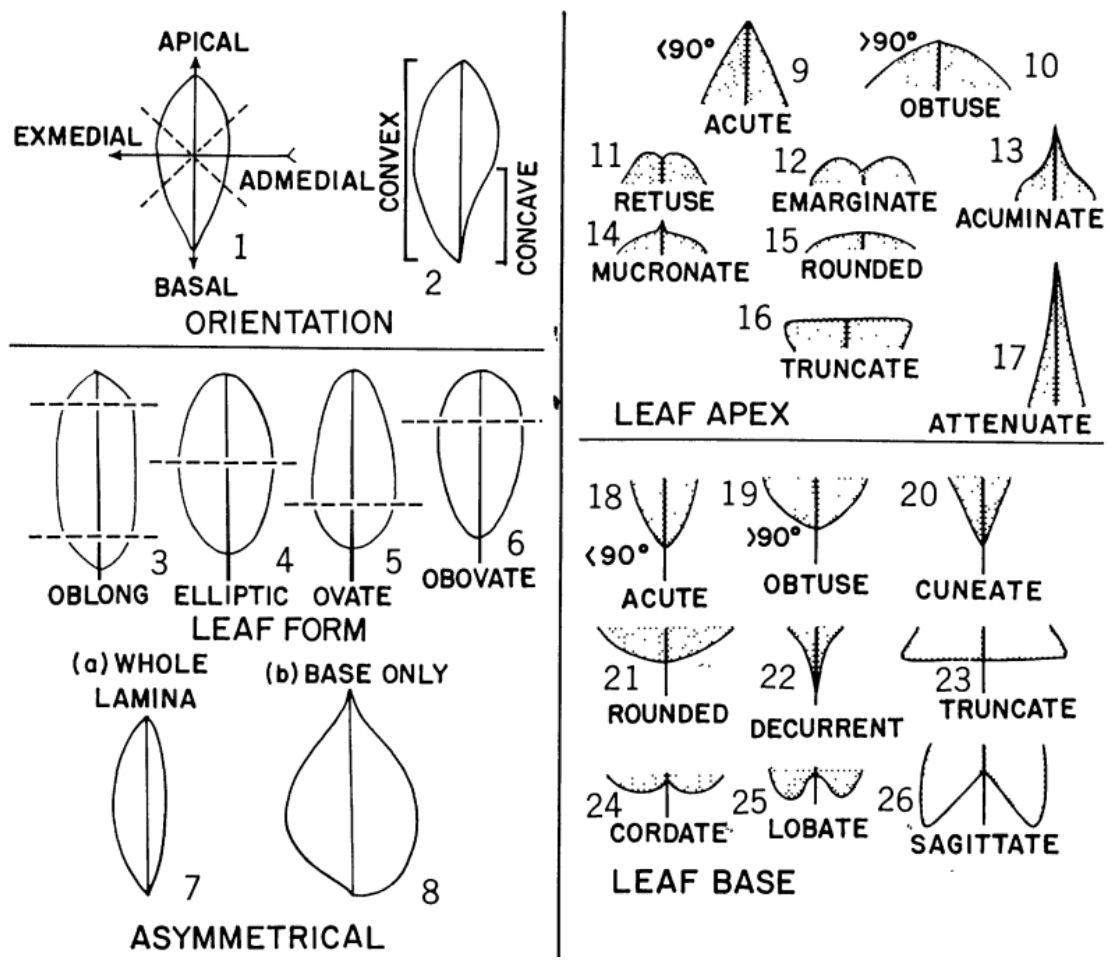

Figure 3.51: Classification of leaf architecture from Hickey (1973, p. 20).

width is closer to the vertical leaf middle. The form changes to ovate for larger leaves. The form of the leaf apex tends to be more acuminate for larger leaves than it is the case for 'Max 1'. The leaf base form ranges from acute to obtuse to rounded to slightly cordate, changing in this order with increasing leaf size. The cordate feature though is not as distinctive as for 'Max 1'. Leaves collected from trees of clone 'AF2' display an ovate leaf form and an acuminate leaf apex for all sizes. The leaf base is mostly truncate with some rounded exceptions for smaller leaves. Bigger leaves have a truncate to cordate leaf base. The cordate property becomes more definitive with increasing leaf size.

\section{Shape Parameters}

Figure 3.52 contains the ratios/shape parameters for the support points defined along the leaf contour. 


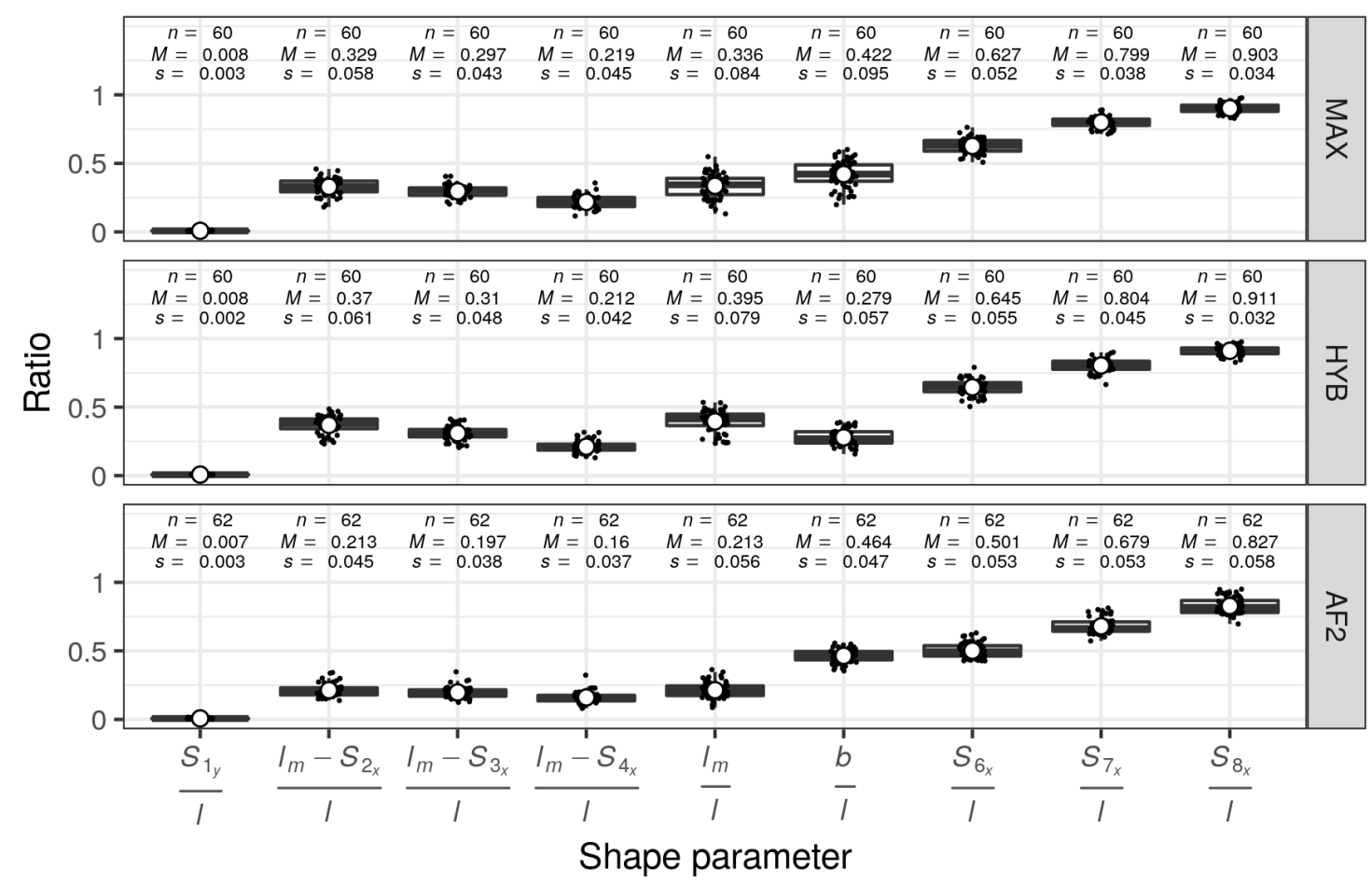

Figure 3.52: Distribution of values of leaf shape parameters by height, clone and section.

The proportion of the radius of the petiole at the leaf base to the overall length $\left(\frac{S_{y}}{l}\right)$ shows a small standard deviation per clone and between clones, exhibiting a mean ratio of 0.007 to 0.008 and standard deviations of 0.002 to 0.003 . The ratios incorporating the $x$-values of contour points $S 2, S 3$ and $S 4$ decrease in their mean value in the given order. This decrease is most distinctive for clone 'Hybride 275' followed by 'Max 1' and least distinctive for 'AF2' which also has the lowest average ratio for the given points. This might in part be explained by the lowest mean $\frac{l m}{l}$ ratio for clone 'AF2'. This points towards lower vertical positions of the greatest width which implies a more ovate leaf form. Leaves from 'Hybride 275' exhibit a higher mean value for this ratio which points towards a more elliptic leaf shape. 'Max 1' is intermediate in these properties. This confirms the observations made in figure 3.50. For the ratio of width to length of leaf blade $\left(\frac{b}{l}\right)$ the order is reversed. Clone 'Hybride 275' has the lowest average ratio, 'Max 1' displays higher values while the highest values are obtained by 'AF2'. This again can be reproduced from visual assessment of the leaf shapes. The 
ratios including the $x$-values from the 3 upper contour points $S_{6}$ to $S_{8}$ show a similar sequence for all clones, but again the mean values establish a ranking. 'Hybride 275' has the highest average ratios for all 3 contour points, followed by 'Max 1' and 'AF2' with the smallest ratios. This is based on the pronounced acuminate leaf apex shape of 'AF2', which implies greater distances between $S_{8_{x}}$ and $l$ (being $S_{9_{x}}$ ).

Following the procedure described in section 3.1.6, the model in 3.2 was parameterized separately for each ratio as the response. Table 3.38 contains statistics for goodness of fit and predictive power of the 9 different linear model fits.

Table 3.38: Goodness of fit and predictive power for different linear models for estimating ratios containing coordinates of support points.

\begin{tabular}{lcrrrrrrr}
\hline Response & $\hat{\sigma}$ & $R S S_{\text {Null }}$ & $d f_{\text {Null }}$ & $R S S_{\text {Model }}$ & $d f_{\text {Model }}$ & \multicolumn{1}{c}{$F$} & \multicolumn{1}{c}{$p$} & $R_{\text {adj }}^{2}$ \\
\hline$\left(\frac{S_{1_{y}}}{l}\right)^{-0.2}$ & 0.172 & 6.169 & 181 & 5.189 & 176 & 6.6 & $<0.001$ & 0.13 \\
$\frac{l_{m}-S_{2_{x}}}{l}$ & 0.048 & 1.357 & 181 & 0.407 & 178 & 138.4 & $<0.001$ & 0.69 \\
$\frac{l_{m}-S_{3_{x}}}{l_{m}-S_{4_{x}}}$ & 0.043 & 0.804 & 181 & 0.333 & 179 & 126.8 & $<0.001$ & 0.58 \\
$\frac{l}{l}$ & 0.041 & 0.433 & 181 & 0.307 & 179 & 36.5 & $<0.001$ & 0.28 \\
$\sqrt{\frac{l_{m}}{l}}$ & 0.050 & 1.726 & 181 & 0.444 & 178 & 171.0 & $<0.001$ & 0.74 \\
$\frac{b}{l}$ & 0.067 & 1.990 & 181 & 0.796 & 178 & 89.0 & $<0.001$ & 0.59 \\
$\frac{S_{6_{x}}}{l}$ & 0.045 & 1.261 & 181 & 0.366 & 178 & 145.4 & $<0.001$ & 0.71 \\
$\frac{S_{7 x}}{l}$ & 0.043 & 0.990 & 181 & 0.330 & 178 & 118.5 & $<0.001$ & 0.66 \\
$\frac{S_{8 x}}{l}$ & 0.042 & 0.594 & 181 & 0.310 & 178 & 54.5 & $<0.001$ & 0.47 \\
\hline
\end{tabular}

For estimating the ratio $\frac{S_{y_{y}}}{l}$ the full model was parameterized. The estimation of the parameter for the Box-Cox transformation suggested a power of -0.2, which visibly eliminated problems with the model error. The $p$-value for predictor $l$ was above the chosen significance level of 0.05 , but omitting this covariate led to a significantly worse model fit in comparison to the full model. Hence the variable was kept here. The remaining models could rely on fewer covariates since the coefficient for the interaction term was never significantly different from 0 . This also was the case for length within the 2 models with response $\frac{l_{m}-S_{3_{x}}}{l}$ and $\frac{l_{m}-S_{4_{x}}}{l}$. This implies that the use of the clone means for these ratios is sufficient for estimation. Modeling the ratio of the position of maximum width $l_{m}$ to length $l$ necessitated a square root transformation of the response to correct for violations of model assumptions. For estimating the ratio of maximum width to length $\left(\frac{b}{l}\right)$ the length may appear to be of negligible influence on the ratio. As it was the case for the first 
ratio, omitting the covariate led to significantly poorer model performance. Again this parameter was kept. Goodness of fit, as assessable by the adjusted coefficient of determination $R_{a d j}^{2}$, ranged from 13 to $74 \%$ of the response variance explained by the predictors. Lower $R_{a d j}^{2}$-values like 0.13 and 0.28 appear to be rather unfavorable. It has to be taken into consideration that the overall variance of the response is rather small for some ratios (compare 3.52). Therefore the predictive ability of these models should be satisfactory.

Figure 3.53 visualizes the correlation of the different ratios and leaf blade length. Resulting lines and curves from the model fit, if length was kept as a predictor after variable selection, are displayed as well.

The location of the regression lines and curves underlines the observations from figure 3.52. The clone wise intercept points towards larger differences between clone 'Hybride 275' and 'AF2', while 'Max 1' inherits intermediate values and hence positioning. In most cases the intercept for 'Max 1' tends towards the one of 'Hybride 275'. This is consistent with some of the findings from the leaf structure measurement in section 3.2.9. The full model functions for all ratios can be derived from the coefficients in the model summaries in Table 3.39 and then used for implementation in the XL model within GrolMP. 

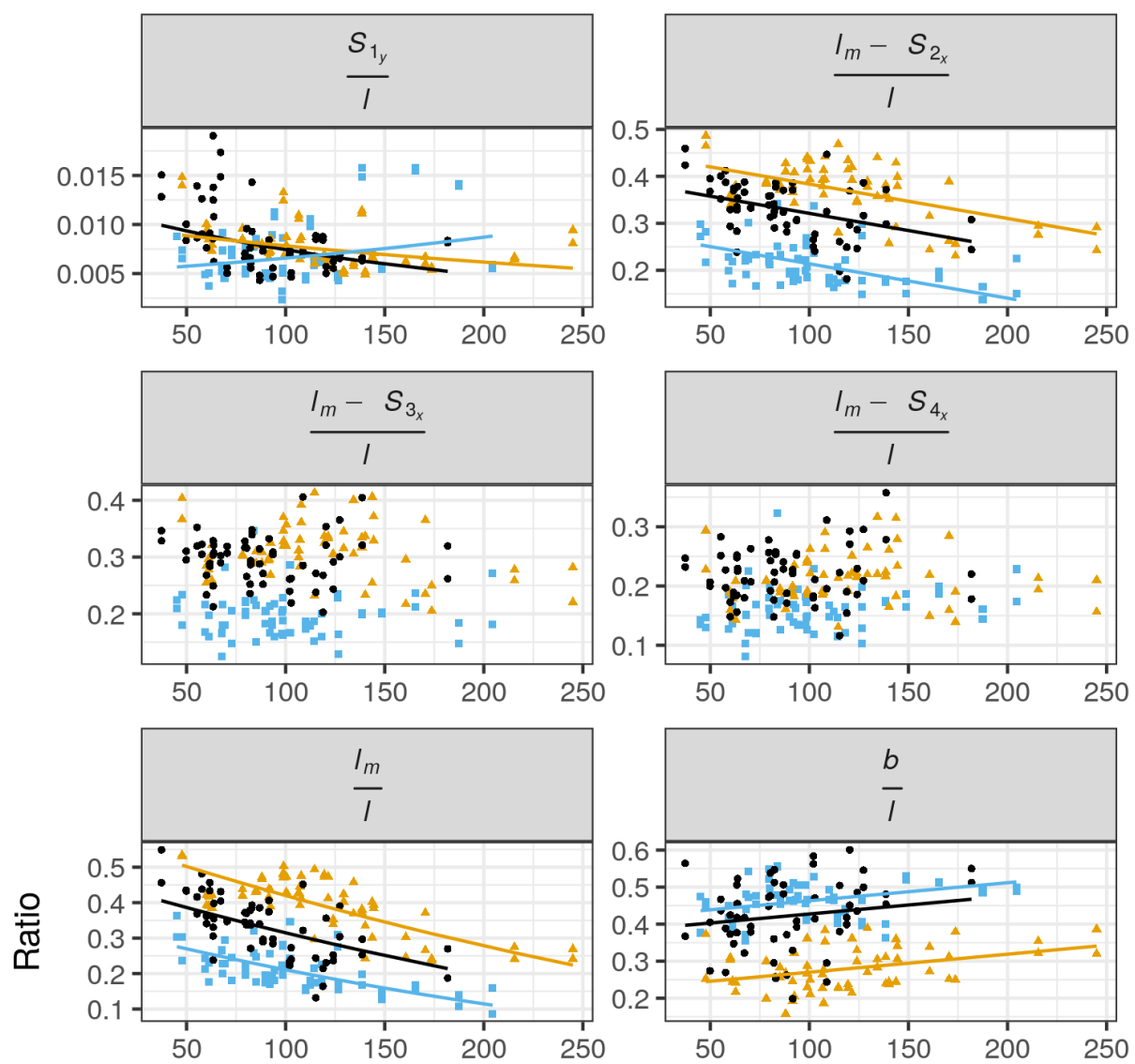

Clone
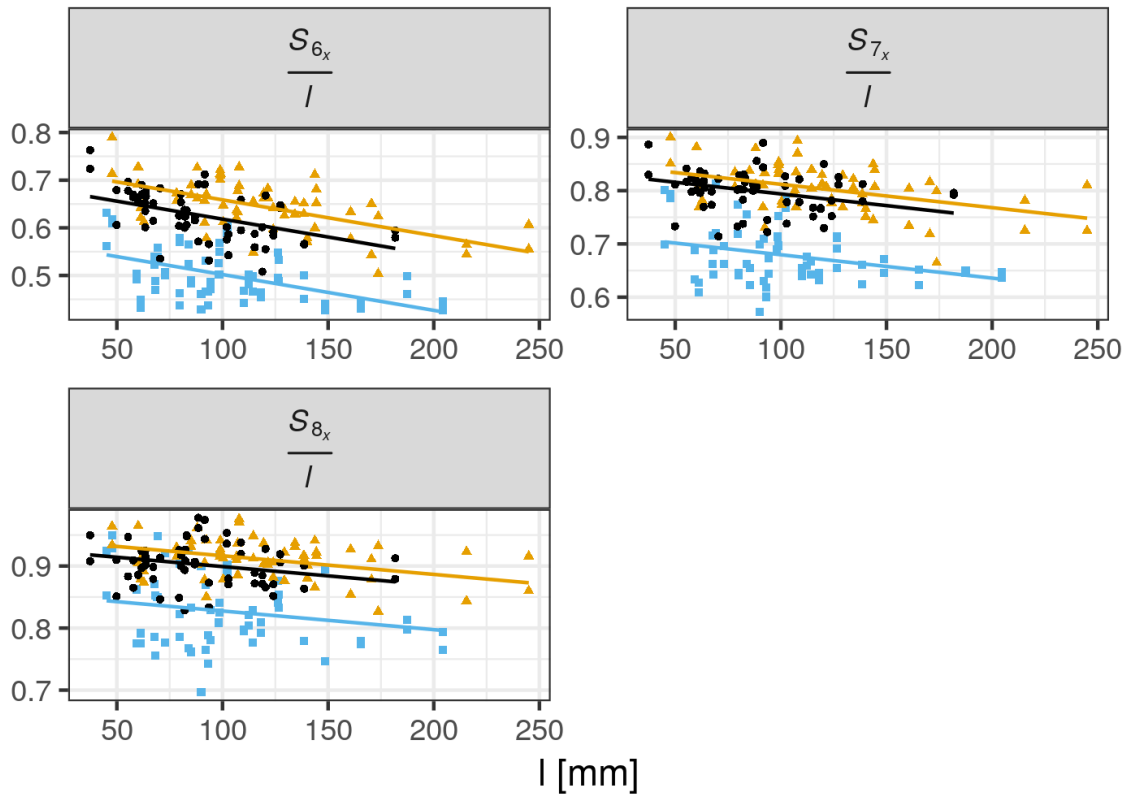

Figure 3.53: Different ratios by leaf blade length. Colors and shapes indicate different clones. If the corresponding linear regression model indicated a significant influence of length, the lines for these models were added to the graph. If the response was transformed, curves resemble the back transformed functions. 
Table 3.39: Summary data for different linear models for estimating ratios containing coordinates of support points. The parameterized model function for the first model is given in Equation 3.20.

\begin{tabular}{|c|c|c|c|c|c|}
\hline Response & Covariate & Est. & se & $t$ & $p$ \\
\hline$\left(\frac{S_{1_{y}}}{l}\right)^{-0.2}$ & $\begin{array}{l}\text { Intercept } \\
l \\
\text { clone } H Y B \\
\text { clone } A F 2 \\
l: \text { clone } H Y B \\
l: \text { clone } A F 2\end{array}$ & $\begin{array}{r}2.42672 \\
0.00238 \\
0.08156 \\
0.45438 \\
-0.00109 \\
-0.00388\end{array}$ & $\begin{array}{l}0.06793 \\
0.00072 \\
0.09360 \\
0.09275 \\
0.00089 \\
0.00093\end{array}$ & $\begin{array}{r}35.7 \\
3.3 \\
0.9 \\
4.9 \\
-1.2 \\
-4.2\end{array}$ & $\begin{array}{r}<0.001 \\
0.001 \\
0.385 \\
<0.001 \\
0.221 \\
<0.001\end{array}$ \\
\hline$\frac{l_{m}-S_{2_{x}}}{l}$ & $\begin{array}{l}\text { Intercept } \\
l \\
\text { clone } H Y B \\
\text { clone } A F 2\end{array}$ & $\begin{array}{r}0.39431 \\
-0.00073 \\
0.06231 \\
-0.10734\end{array}$ & $\begin{array}{l}0.01041 \\
0.00009 \\
0.00918 \\
0.00874\end{array}$ & $\begin{array}{r}37.9 \\
-7.7 \\
6.8 \\
-12.3\end{array}$ & $\begin{array}{l}<0.001 \\
<0.001 \\
<0.001 \\
<0.001\end{array}$ \\
\hline$\frac{l_{m}-S_{3_{x}}}{l}$ & $\begin{array}{l}\text { Intercept } \\
\text { clone } H Y B \\
\text { clone } A F 2\end{array}$ & $\begin{array}{r}0.29689 \\
0.01340 \\
-0.10004\end{array}$ & $\begin{array}{l}0.00556 \\
0.00787 \\
0.00781\end{array}$ & $\begin{array}{r}53.4 \\
1.7 \\
-12.8\end{array}$ & $\begin{array}{r}<0.001 \\
0.09 \\
<0.001\end{array}$ \\
\hline$\frac{l_{m}-S_{4_{x}}}{l}$ & $\begin{array}{l}\text { Intercept } \\
\text { clone } H Y B \\
\text { clone } A F 2\end{array}$ & $\begin{array}{r}0.21921 \\
-0.00758 \\
-0.05877 \\
\end{array}$ & $\begin{array}{l}0.00535 \\
0.00756 \\
0.00750 \\
\end{array}$ & $\begin{array}{l}41.0 \\
-1.0 \\
-7.8 \\
\end{array}$ & $\begin{array}{r}<0.001 \\
0.317 \\
<0.001 \\
\end{array}$ \\
\hline$\sqrt{\frac{l_{m}}{l}}$ & $\begin{array}{l}\text { Intercept } \\
l \\
\text { clone } H Y B \\
\text { clone } A F 2\end{array}$ & $\begin{array}{r}0.68191 \\
-0.00121 \\
0.08687 \\
-0.10215\end{array}$ & $\begin{array}{l}0.01087 \\
0.00010 \\
0.00960 \\
0.00913\end{array}$ & $\begin{array}{r}62.7 \\
-12.2 \\
9.1 \\
-11.2 \\
\end{array}$ & $\begin{array}{l}<0.001 \\
<0.001 \\
<0.001 \\
<0.001\end{array}$ \\
\hline$\frac{b}{l}$ & $\begin{array}{l}\text { Intercept } \\
l \\
\text { clone } H Y B \\
\text { clone } A F 2\end{array}$ & $\begin{array}{r}0.37869 \\
0.00049 \\
-0.15726 \\
0.03576\end{array}$ & $\begin{array}{l}0.01455 \\
0.00013 \\
0.01284 \\
0.01222\end{array}$ & $\begin{array}{r}26.0 \\
3.7 \\
-12.2 \\
2.9\end{array}$ & $\begin{array}{r}<0.001 \\
<0.001 \\
<0.001 \\
0.004\end{array}$ \\
\hline$\frac{S_{6_{x}}}{l}$ & $\begin{array}{l}\text { Intercept } \\
l \\
\text { clone } H Y B \\
\text { clone } A F 2\end{array}$ & $\begin{array}{r}0.69351 \\
-0.00075 \\
0.04043 \\
-0.11629\end{array}$ & $\begin{array}{l}0.00986 \\
0.00009 \\
0.00870 \\
0.00828\end{array}$ & $\begin{array}{r}70.3 \\
-8.4 \\
4.6 \\
-14.0\end{array}$ & $\begin{array}{l}<0.001 \\
<0.001 \\
<0.001 \\
<0.001\end{array}$ \\
\hline$S_{7 x}$ & $\begin{array}{l}\text { Intercept } \\
l \\
\text { clone } H Y B \\
\text { clone } A F 2\end{array}$ & $\begin{array}{r}0.83772 \\
-0.00044 \\
0.01810 \\
-0.11415\end{array}$ & $\begin{array}{l}0.00937 \\
0.00009 \\
0.00827 \\
0.00788\end{array}$ & $\begin{array}{r}89.4 \\
-5.2 \\
2.2 \\
-14.5\end{array}$ & $\begin{array}{r}<0.001 \\
<0.001 \\
0.03 \\
<0.001\end{array}$ \\
\hline$\frac{S_{8_{x}}}{l}$ & $\begin{array}{l}\text { Intercept } \\
l \\
\text { clone } H Y B \\
\text { clone } A F 2\end{array}$ & $\begin{array}{r}0.92921 \\
-0.00030 \\
0.01742 \\
-0.07161\end{array}$ & $\begin{array}{l}0.00907 \\
0.00008 \\
0.00801 \\
0.00762\end{array}$ & $\begin{array}{r}102.4 \\
-3.6 \\
2.2 \\
-9.4\end{array}$ & $\begin{array}{r}<0.001 \\
<0.001 \\
0.031 \\
<0.001\end{array}$ \\
\hline
\end{tabular}




$$
\left(\frac{S_{1_{y}}}{l}\right)^{-0.2}=2.42672+\left(0.00238+\left(\begin{array}{c}
0 \\
-0.00109 \\
-0.00388
\end{array}\right) * \text { clone }\right) * l+\left(\begin{array}{c}
0 \\
0.08156 \\
0.45438
\end{array}\right) * \text { clone }
$$

Using these models the coordinates of the contour points were estimated for each half leaf contour. The euclidean distances between the observed contour point and the estimated counterpart were calculated and their distribution is displayed point wise in figure 3.54 separated by clone.

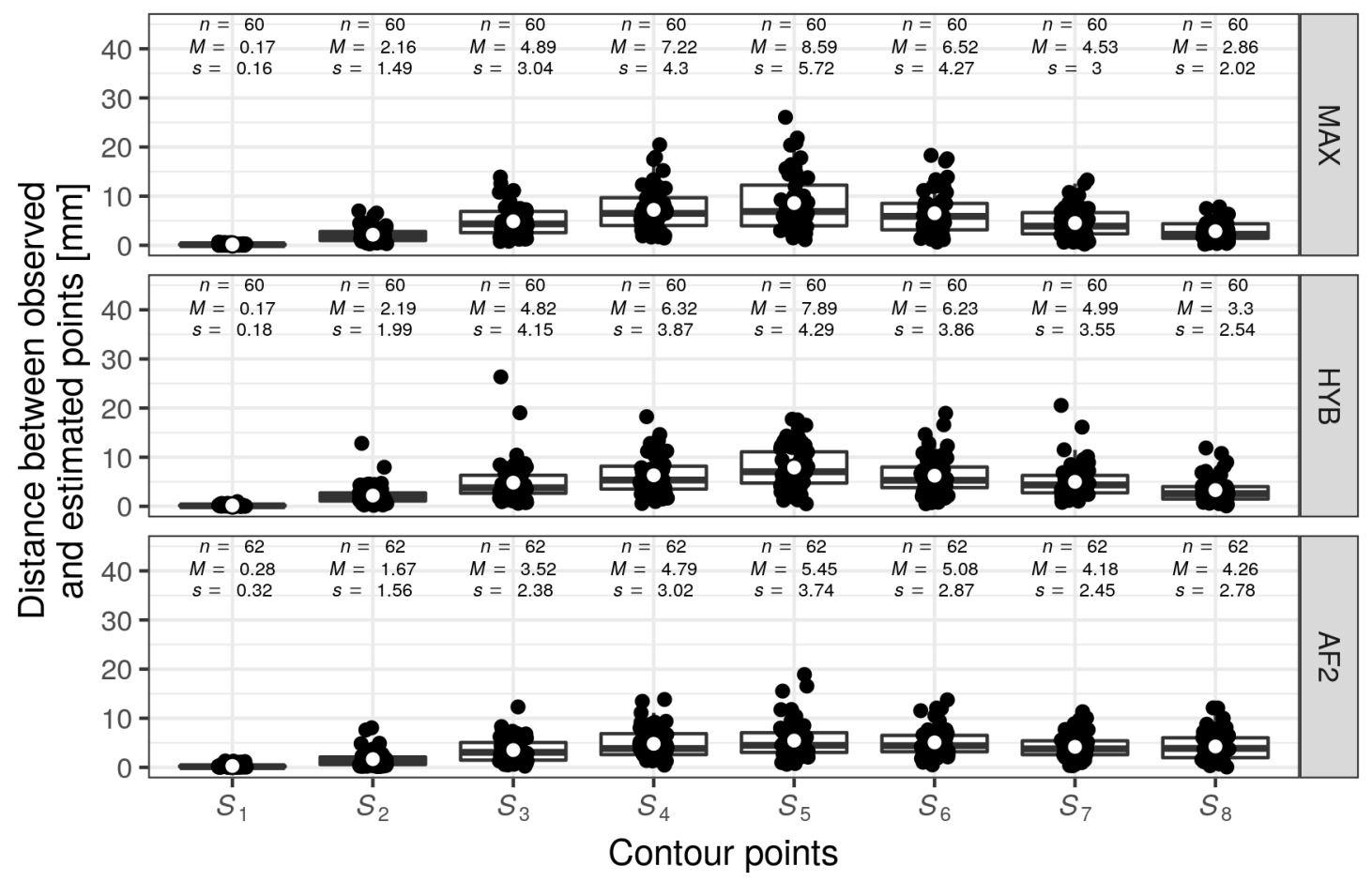

Figure 3.54: Distribution of euclidean distance between observed and estimated contour points separated by clone.

Starting at the base, the mean deviations for all 3 clones rise with the points along the contour and culminate for the coordinates of $S_{5}$. They then decrease again towards the leaf blade tip. The maximum mean values for the deviation range from 5.45 to $8.59 \mathrm{~mm}$, the latter being the deviation for $S_{5}$ of all 'Max 1' contours. The single value maximum deviation reaches up to $25 \mathrm{~mm}$. All in all 
these deviations seem bearable. When further utilizing these estimated contour point coordinates with the procedure described in 3.1.6 the parametric curve can be calculated. The area enclosed by the digitized and estimated leaf half contour can then be compared (see Figure 3.55). The bisector is drawn here as a line for visual orientation. All points being placed perfectly on the line would imply a Pearson's correlation $r$ equaling 1 . The actual values are given in each panel.

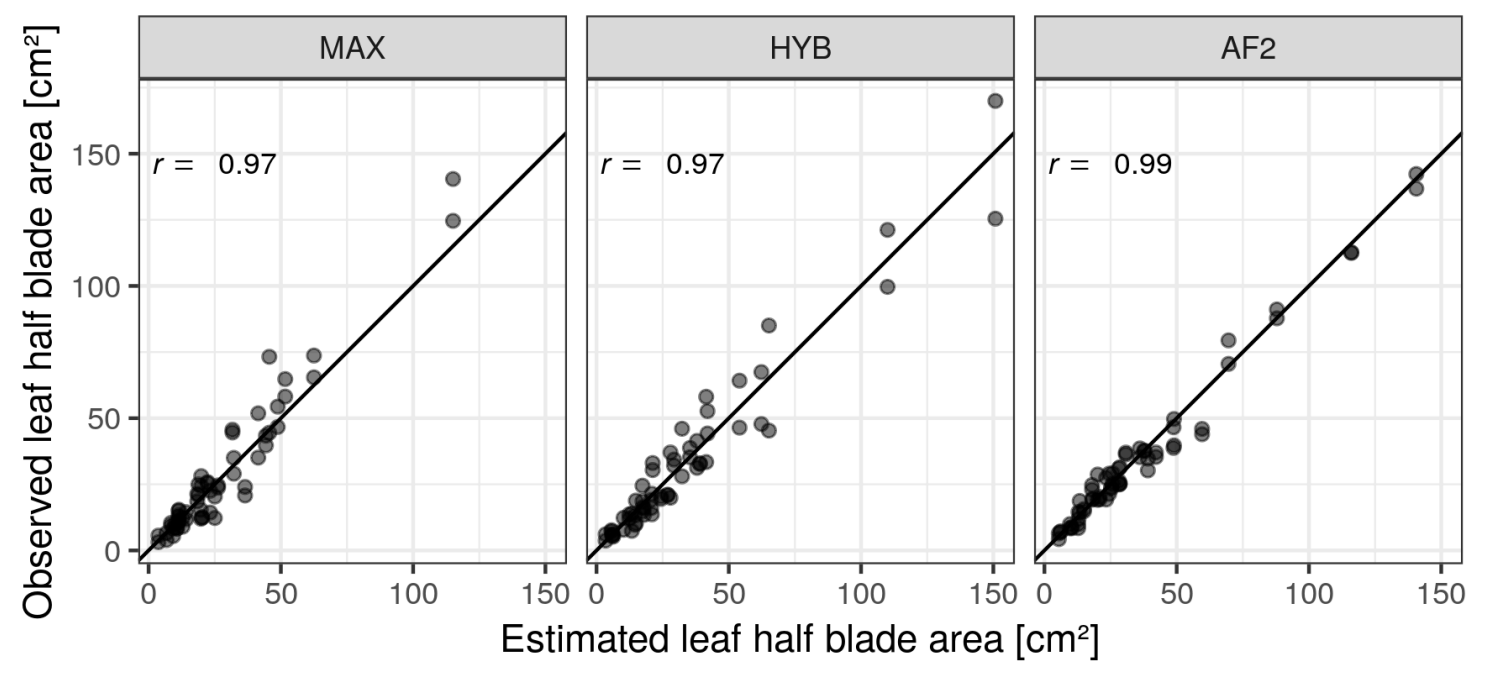

Figure 3.55: Comparison of observed and estimated values for leaf half area separated by clone. The black solid line is the bisector. In each panel the corresponding Pearson correlation coefficient $r$ is given.

Using this display for assessing the goodness of fit the overall impression is very good. All correlations between observed and estimated area of leaf half are well above 0.95. No bias is observable.

Figure 3.56 contains a similar comparison but for contour length. The impression here is quite different: While the correlation coefficients are again quite high (ranging from 0.97 to 0.99 ), there is clearly a bias present. The observed contour lengths are consistently higher than the estimated ones. The differences increase with increasing observed contour length, with the maximum deviation reaching $1.5 \mathrm{~cm}$. 


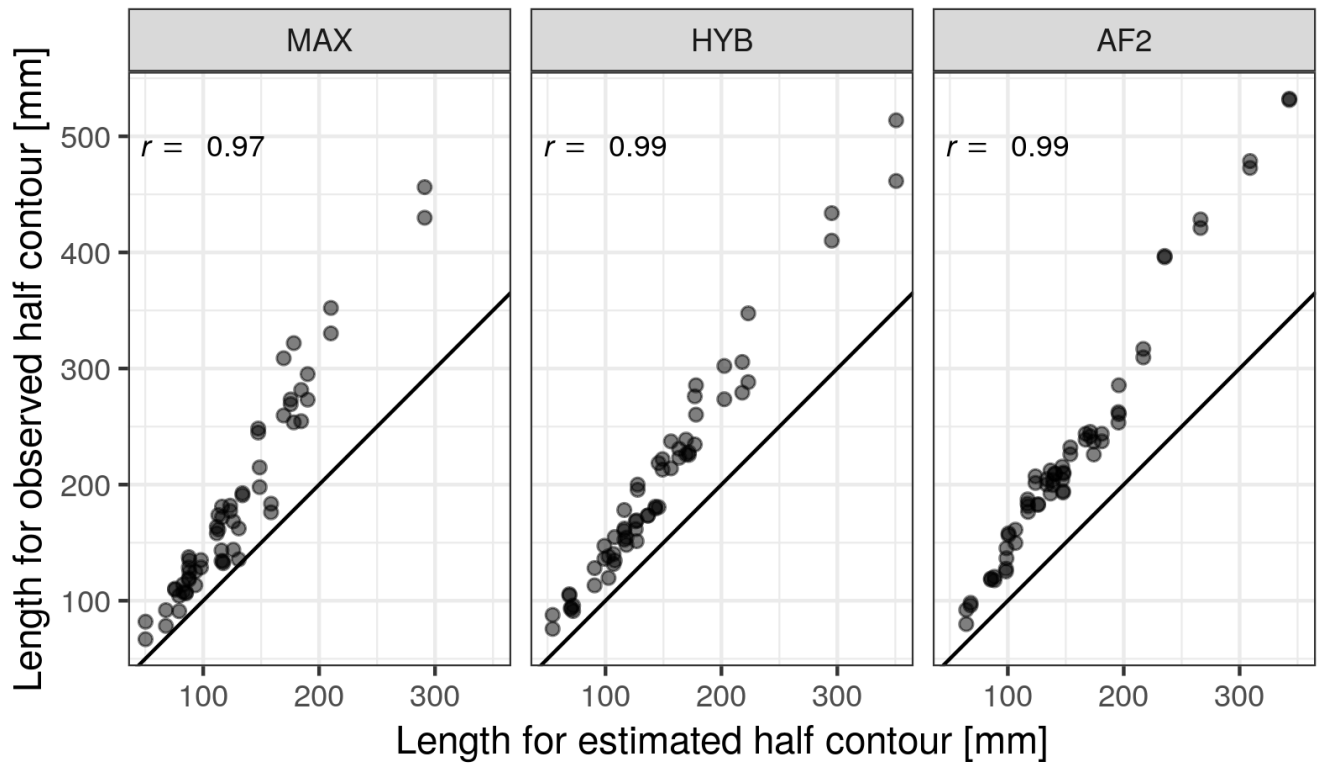

Figure 3.56: Comparison of observed and estimated values for leaf half contour length separated by clone. The black solid line is the bisector. In each panel the corresponding Pearson correlation coefficient $r$ is given.

Implicitly the chosen procedure for leaf shape estimation underestimates the contour length, which is trivial since the serration of the leaf margin was not considered after calculating the parametric curve. While this bias is of course unfavorable, its impact remains rather marginal since the serration does not affect the leaf blade area or shape to a greater extent. Henke et al. (2014) added the parameterization of a Fourier-series approximation of the saw-tooth function to the shape coordinates. This was tested for the digitized leaves here but did not yield satisfying results. Since Henke et al. (2014) describe this as a rather visual enhancement, which might be of more use for more serrated leaf margins (which in comparison is not the case here), this step was dropped from modeling.

\subsubsection{GrolMP Implementation}

With all models parameterized as described, the next step can be made to implement the functions in GrolMP. In Figure 3.57 an overview in the form of an informally designed flowchart is given on the modeling procedure containing the used modules, constants, variables and methods as well as how these work to- 
gether and in what sequence the model operates with these. The instruction on how to obtain the full source code of the model with comments is given in the Appendix.

The modules represent the tree organs that are needed to model the tree structure. The graphical representation of the module Bud is a sphere with a radius of $1 \mathrm{~mm}$. The attributes of each bud contain the identifier of the tree that the bud belongs to (treeID), the rotation year it developed in ( $t$ ), the branch order (order also ord), if it belongs to a main or minor stem (main), if it is an apical or lateral bud (proLon), the length of the GU that it belongs to (guL), its relative position on that GU (relPos, as a ratio) and its height in relation to tree height (relH, as a ratio). The module Internode is visualized by a cylinder ( $F$ ). Additionally to the already established attributes (same as for bud, if present) the length and diameter of the cylinder are specified as well as the color. The color is set accordingly to the year that the internode developed in for easier visual distinguishing. The module Sylleptic has no graphical representation and rather is a helper module since it gets replaced by a shoot within the growth method which will be explained later. The Tip module has no graphical representation as well and is, again, a helper module for the relative height calculation. The module Leaf is represented by the GrolMP function leaf $3 \mathrm{~d}$ which accepts a pointlist array as input and turns it via triangulation into a mesh node. The module Petiole is represented by a cylinder like the internodes but only requires the specification of length and diameter. After defining the modules, a manual input has to be made for specifying the clone as an constant integer value CLONE with $0=$ 'Max 1', 1 = 'Hybride 275' and 2 = 'AF2'. The constants ROW and TREESPROW specify the number of rows and trees per row respectively. In the following paragraphs the procedure of the model by sequence of and applying rules and calling functions is explained. This is done sequentially in the order of the model code. It should be kept in mind though that the rules are being applied parallel. A further remark has to be made that all functions with any metrically scaled variable were parameterized with data on the [mm] scale. The visual output of GrolMP works better for variables in the [m] range therefore all input and output to and from functions was properly scaled within.

The init method defines the initial state of the model with the Axiom node being replaced by the buds on the stool. The global integer variable year is set to 0 . The number of axes axN and their directional angle gets estimated with the 


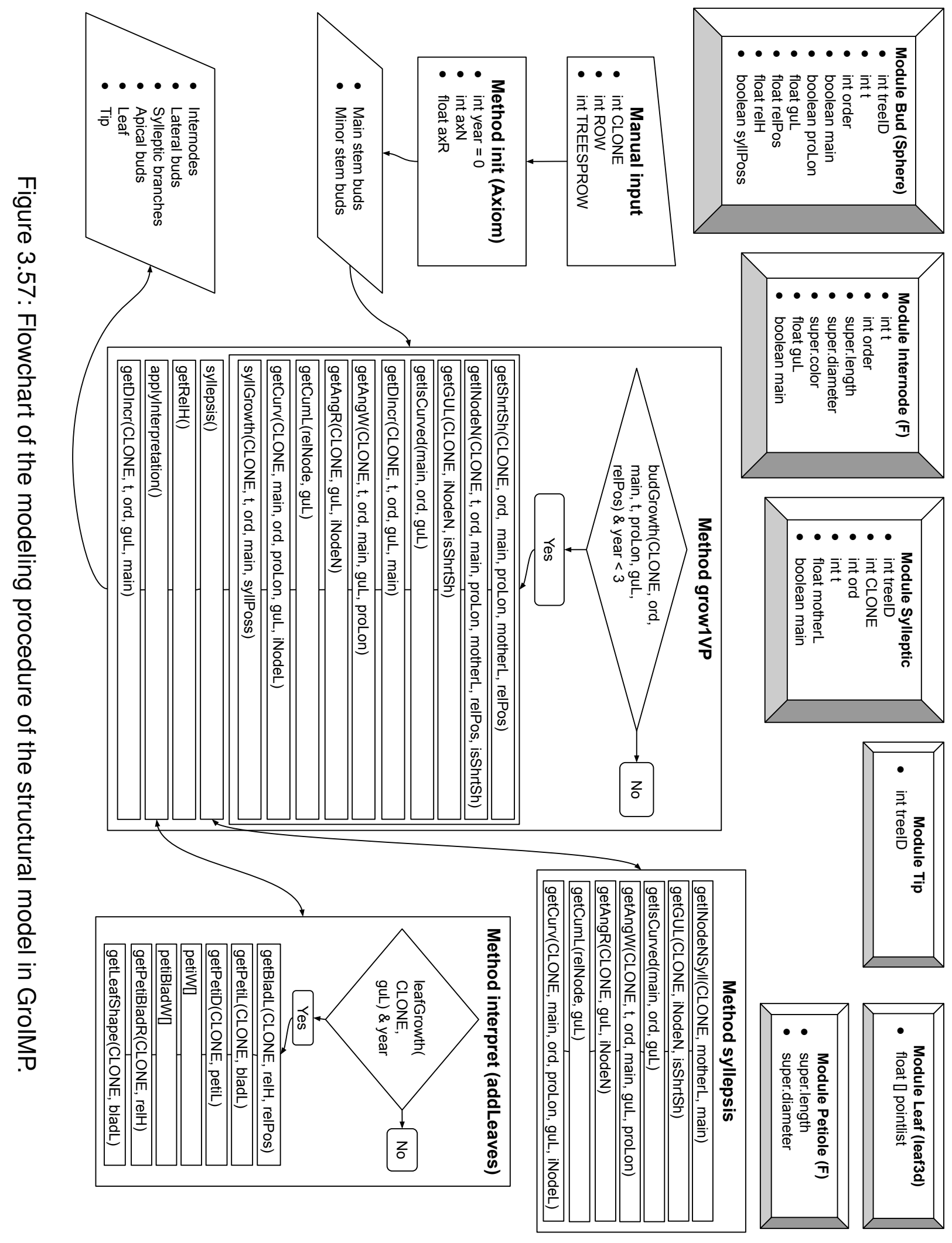


corresponding functions which is a Poisson random number generator based on mean value 8.82 for axN and the function taken from Table 3.6. The bud of the main stem facing upward from the $x y$-plane and the initial buds of the side stems with their directional angle axR are generated. The whole procedure is repeated based on the number of trees and each set of buds is placed separately via the Translate function which establishes the between and within row distances of $1.8 \mathrm{~m}$ and $0.5 \mathrm{~m}$.

With the buds of main and minor stems generated for each tree as the initial state, the grow1VP method can be executed now which applies all rules needed to simulate the growth cycle of one vegetation period. Before applying the replacement rule for all buds it needs to be considered if the bud will grow at all. This gets estimated via the budGrowth function which distinguishes between the cases initial buds, buds on the main axis (order 0 ) of each stem as well as apical and lateral buds. For the former two cases buds always grow as already established. For the latter two, the probability of the bud breaking dormancy gets estimated based on functions with the coefficients from Table 3.8 and 3.9. Utilizing this probability, a binomial random number generator determines if the bud will be replaced by a new shoot or not. Furthermore, after the rotation year 3 the replacement rule will not be applied and a short message is printed to the console that the end of the rotation has been reached. If growth will commence a sequence of several functions is called. The first getShrtSh determines whether a short shoot will develop or not (function based on Table 3.13). This has influence on the function getINodeN since it distinguishes between the following cases (Tables with respective coefficients given in brackets): GUs from the first rotation year (Table 3.10), prolongation GUs (Table 3.11) as well as lateral long or short shoots (Table 3.15 and 3.14). The latter two relate only to regular shoots. The distinction of regular and sylleptic shoots is also important for the estimation of GU length based on the number of internodes by getGUL. For long shoots a function is formulated using the coefficients from Table 3.16, for short shoots the length equals the number of internodes in $\mathrm{mm}$. As established by the generalized linear model described in Table 3.26, the two cases if a GU is curved or not are being distinguished. The getAngw function then generates the branching angle of the to be developed GU. The directional angle is already given and the new shoot inherits the angle from the bud that is being replaced. The lateral buds of the new shoot however 
need directional angles to be assigned and the phyllotaxis is given by getAngR conditional on the GU being a short shoot (see Table 3.37) or a long shoot (see evaluation of phyllotaxis in section 3.2.5. So far all functions were concerned with estimating variables on the GU level that are invariant within the GU. The directional angle is written to an array whose length equals that of the number of nodes and the angle is multiplied with the indices of the array. Within a for loop with a number of iterations equal to the number of internodes the new shoot consisting of internodes, lateral buds or sylleptic shoots is generated. With each iteration and hence for each internode and its relative rank the internode length is estimated by subtracting the cumulative length of the prior internode from the current cumulative length (see model in Table 3.17). For the first iteration and if the GU is not a prolongation shoot, the branch angle is added. For the following internodes, the curvature angle is estimated based on the internode length and other variables as described in the model summary in Table 3.27. The curvature angle is added after the internode has been generated but only if the GU has been determined as curved and not after the last internode. After each internode a branch in the graph is defined with the angR phyllotaxis rotation and the placement of either a bud or a sylleptic shoot. The latter is determined by the syllGrowth function based on the model presented in Table 3.24. After the for loop is finished with generating all internodes and lateral buds or sylleptic shoots the apical bud is generated. This concludes the replacement rule of each bud. Next, invoking the syllepsis method via the apply iterator once, replaces the sylleptic modules with sylleptic shoots. The procedure and the utilized function for generating a sylleptic shoot are the same as for regular shoots with the only exception of internode count (function from model summarized in Table 3.25). Furthermore, the Boolean attribute syllPoss gets switched to false for all buds generated within the syllepsis method. As described in section 3.2.7, this is a necessary constraint to prevent sylleptic shoots from having any sylleptic children. In the same manner as the syllepsis method, the getRelH method is invoked. This updates the relative height of all buds by calculating the ratio of the bud's current height ( $z$-coordinate) to the maximum height per tree which is also the maximum $z$ coordinate for the Tip modules per tree. The latter is obtained via a query which searches for the maximum height value of all Tip modules with the same treeID as the considered bud. Theoretically, it is possible to have the query search 
through all buds with the same treeID for the maximum height. Even if this is restricted to apical buds on the main stem with branch order 0 this takes some time to compute. Using the Tip module as a helper reduced the computation time for the grow1VP method noticeably. Adding the leaves is implemented through an interpretive rule. Within the same generative step, this adds an additional derivation whose result is then interpreted geometrically (Kurth, 1994a; Kniemeyer, 2008). The application of the interpretive rule entails, first, the estimation if a leaf is going to develop or not which can be the case if the bud has developed during the current year and the binomial random number generator (based on the model described in Table 3.28 has a positive outcome (success). If the latter indicated leaf growth the leaf morphology parameters are estimated. This begins with the central size and shape variable leaf blade length via getBladL which is the implementation of the model in Table 3.29. The lamina length is in turn used for estimating the petiole length (model from Table 3.31) which then itself serves, with clone, as the predictor for the petiole diameter (model from Table 3.32). The orientation angle of the petiole relative to the shoot and the angle between leaf blade and petiole are input as clonal mean values (models from Table 3.33 and 3.34). The last parameter for determining the leaf orientation is the rotation angle between petiole and leaf blade which is estimated by getPetiBladR (see Table 3.35). The final step is simulating the leaf shape which is implemented through the getLeafShape function. Based on the leaf blade length, the coordinates for the 8 support points are estimated with the function for each clone summarized in Table 3.39. The coordinates for the half contour support points are then mirrored along the $x$-axis which is why the generated leaves are all symmetrical. The full contour support points coordinates are then written to a pointlist which is returned by getLeafShape to the leaf $3 \mathrm{~d}$ function. It was decided to not fully implement the approach by Henke et al. (2014) due to performance reasons. The interpolation procedure with the parametric curve was therefore dropped. This concludes the generation of petioles and leaves. The last method is the diameter increment which updates the diameter of all internodes by the estimated increment but only for internodes that have not lengthened during the current year. The last step of the growVP method is then incrementing the global year variable by 1 . This concludes the simulation of the growth cycle of 1 vegetation period. The whole procedure can then be repeated until the end of the third growth season. 
Figure 3.58 outlines the development of a single tree through a rotation cycle by the graphical output of the structural model at the end of each vegetation period separated by clones. By visual assessment a satisfactory representation of the general tree structure can be concluded. The branch architecture seems to be approximated quite well which especially includes the curvature. The differences between the main and minor stems are comparably obvious and seem a bit overemphasized. Differences in habitus have also been captured well with a more slender appearance for 'AF2' (third row). Additionally, the general change in foliation distribution over the years (all rows from left to right) is clearly visible although it should be minded here that the functions concerning leaf structure, position and shape were parameterized with data from the third respectively second rotation year and are being applied in all years.

The next Figure (3.59) contains 3 neighboring trees within a row for all clones at the end of the third vegetation period while the leaves have been omitted. The different colors are only kept for easier differentiation of the years that the GUs have developed in. In the first (a) and third panel (c) for 'Max 1' respectively 'AF2' sylleptic shoots can be seen (colored blue). For all clones, the minor stems and branches of each tree clearly extend out of the individual tree's growing space within rows which is $0.5 \mathrm{~m}$. Again, this is less pronounced for 'AF2' due to the increased curvature. The general tendency for all clones which can also be observed in the field seems realistic. A caveat has to be issued here on the fact that the growth in this model (be it bud, shoot, petiole or leaf) does not avoid collisions, so all organs might grow/cross through one another. Furthermore, the differences between the neighboring trees in individual tree height are solely based on stochastic processes although they might produce the illusion of competition.

In Figure 3.60 the prior perspective is rotated in the horizontal $(x y)$ plain so that the view is aligned with the row direction. Between the rows the growing space is $1.8 \mathrm{~m}$ which is not exceeded for the great majority of shoots. The gaps between trees in the upper part of the crown are more conspicuous than for the lower part. This tendency is increased for 'Max 1' and 'Hybride 275' while 'AF2' is visibly more slender, again. While gaps are visible between rows especially during the winter in leafless condition they seem overemphasized here. 


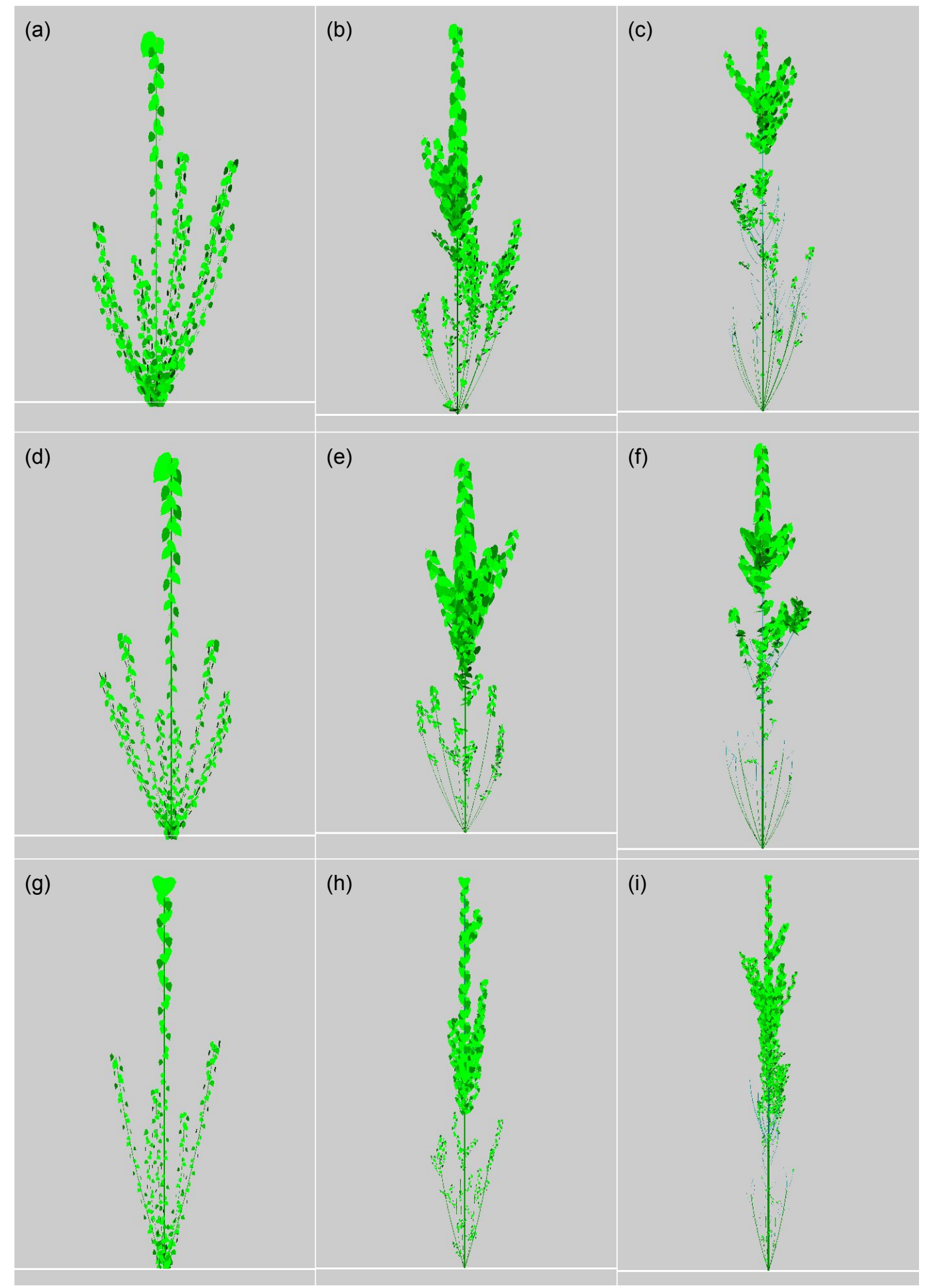

Figure 3.58: Graphical output from the structural model for all 3 clones for a single tree. Each clone is displayed per row ('Max 1' at the top, 'Hybride 275' in the middle and 'AF2' at the bottom) and the columns mark the time steps to the end of each vegetation period ( 3 iteration steps with 1 to 3 from left to right). For example panel (a) shows a 'Max 1' tree at the end of the first vegetation period and panel (c) shows the same tree at the end of the third vegetation period. The images in the panels have been scaled to a comparable size. 


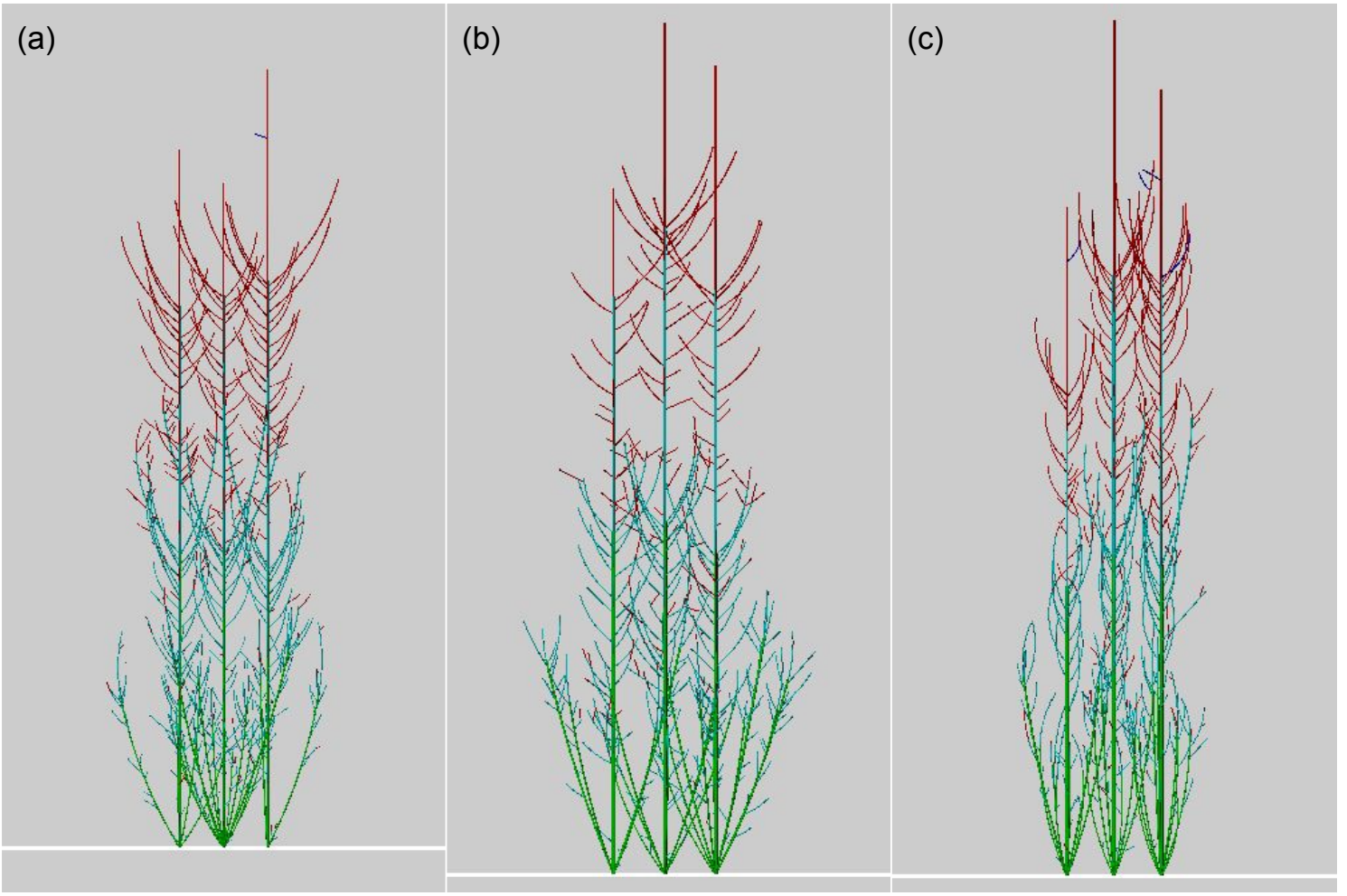

Figure 3.59: Graphical output from the structural model for all 3 clones for 3 neighboring trees in a row at the end of the third vegetation period. The perspective is facing the row orientation orthogonally. The leaves have been omitted from the model and a constant of $10 \mathrm{~mm}$ has been added to the diameter of each growth unit for better visibility. Panel (a) shows 3 trees of 'Max 1', panel (b) shows 'Hybride 275' and panel (c) displays 'AF2'. The images in the panels have been scaled to a comparable size. 


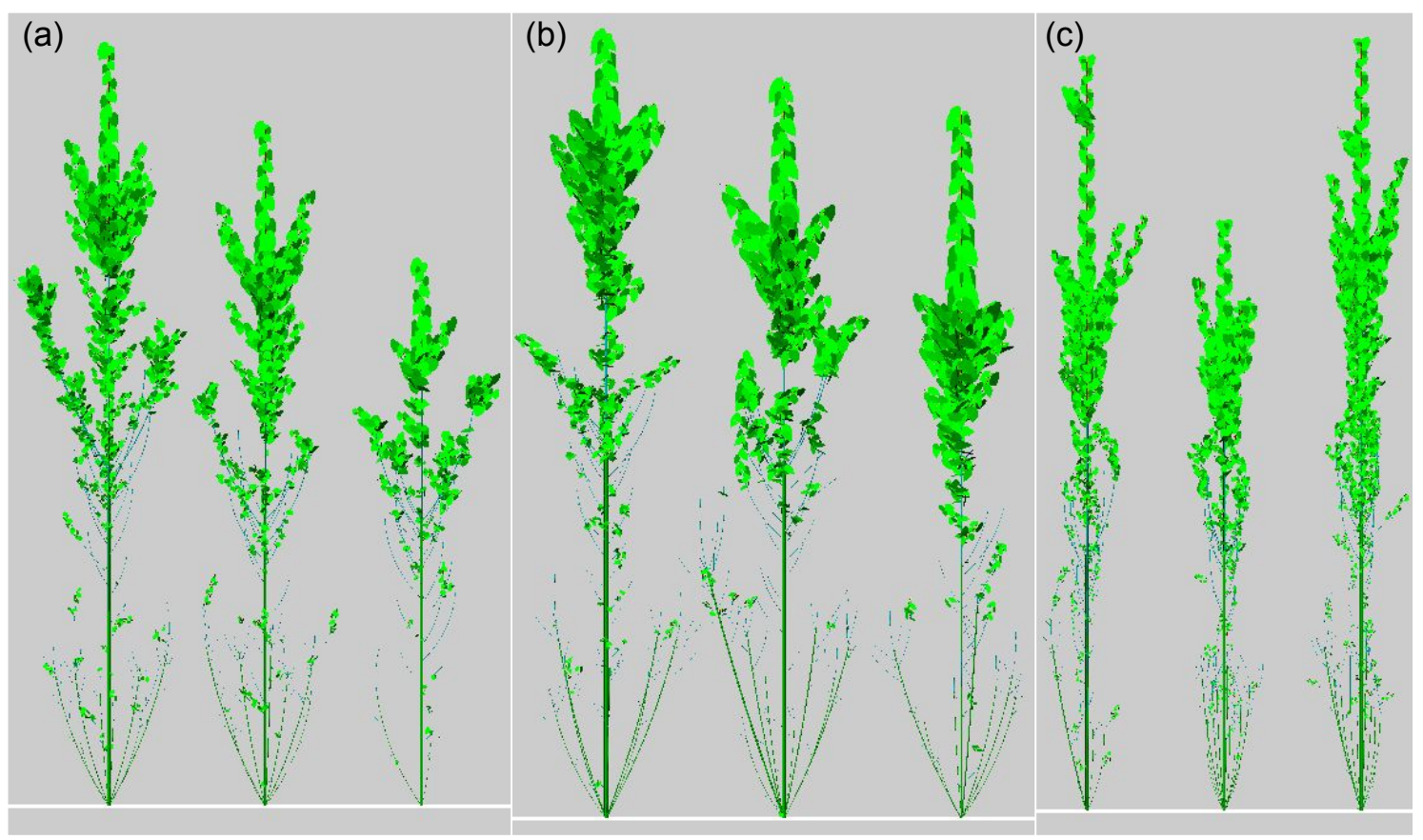

Figure 3.60: Graphical output from the structural model for all 3 clones for 3 neighboring trees between rows at the end of the third vegetation period. The perspective is facing in the row direction. Panel (a) shows 3 trees of 'Max 1', panel (b) shows 'Hybride 275' and panel (c) displays 'AF2'. 
Figure 3.61 exemplifies the development of a whole plantation stand for 15 trees in 3 rows of clone 'Max 1'. The images from the graphical output combine and validate some of the observations made above. Trivially, all trees that were measured were situated within the trial plots and not at the trial border so all trees exhibit an architecture typical for individuals with competition to all sides. The overall impression of the resemblance of this habitus is satisfactory.

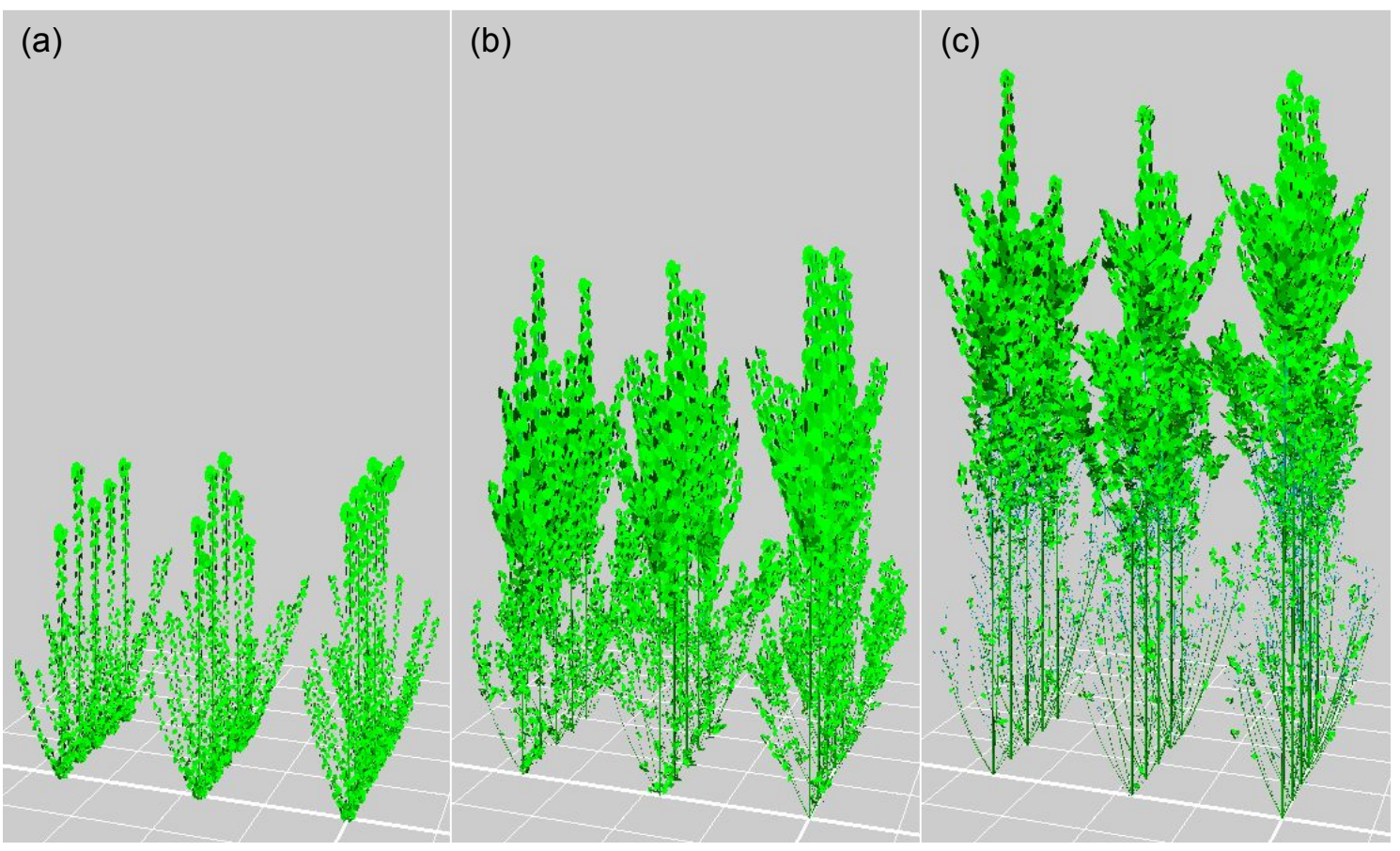

Figure 3.61: Graphical output from the structural model for a small plantation stands of 15 trees in 3 rows for clone 'Max 1'. The panels show the stand at the end of each vegetation period from 1 (a) to 3 (c). 


\section{Interconnection}

\subsection{Statistical models}

Before focusing on the software based implementation of the interconnection between yield and structural model, two additional statistical models had to be parameterized. The analysis of the relationship between internode count and length that was scrutinized by the model described in Table 3.16 is reversed here. The subset from the structural data that was used for the reversed analysis are the GUs from the first year of the second rotation (referred to as $t=1$ in the structural model). This implies that only GUs of the first branch order were used. Figure 4.1 contains the scatter plot that visualizes the correlation of both variables.

The relationship is clearly nonlinear and a positive correlation is visible as it would be expected based on the findings for the structural model. A glm was fitted using the poisson model family (see Table 4.1). The independent variable GU length $l$ was log transformed and entered as a second-degree polynomial. The intercept was not significant which is logical. It was therefore dropped from the model. Incorporating the factor clone into the model did not significantly improve the fit which already has a very high predictive power indicated by $D_{a d j}^{2}=0.99$.

Based on the GU length from the growth simulator, the mean internode count can now be estimated and passed on to a Poisson random number generator. In contrast to the structural model where the length of the GU is estimated based on the internode count, here the internode count is estimated based on the length imported from the XML file, hence the length estimation is obsolete. 


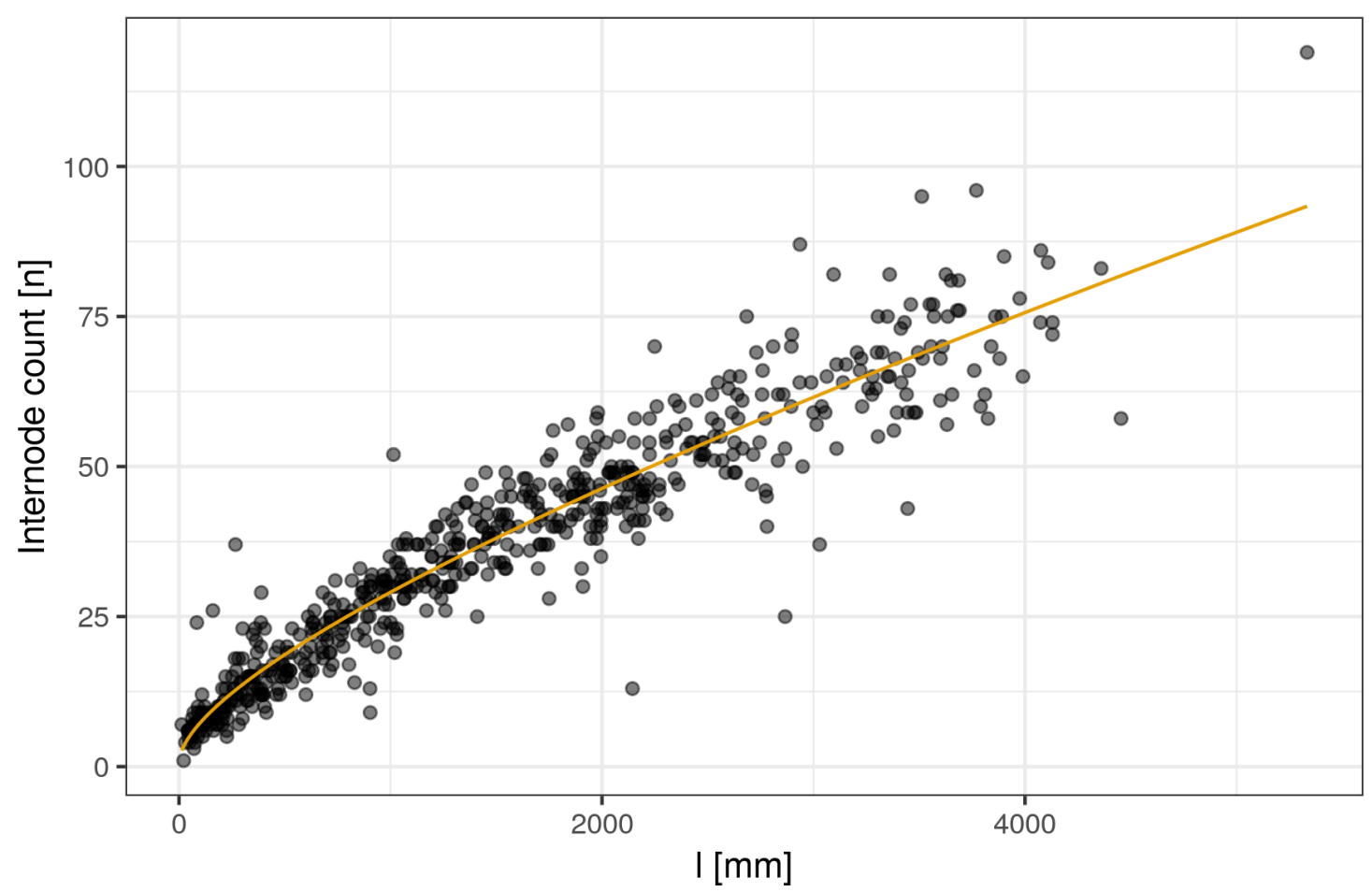

Figure 4.1: Internode count by GU length $l$ for GUs from the first year of the second rotation. This inherently only includes GUs of the first branch order. The yellow line is based on the back transformed estimations of the model described in Table 4.1

Table 4.1: Summary of generalized linear model fit for estimating $\eta$ of the response $i N o d e N$ of count of internodes for GUs from the first year of the second rotation. The independent variable $l$ stands for the GU length.

\begin{tabular}{|c|c|c|c|c|c|c|c|}
\hline Model (link) & $D_{\text {Null }}$ & $d f_{\text {Null }} \quad L$ & $D_{\text {Model }}$ & $d f_{\text {Model }}$ & & $x^{2}$ & $p$ \\
\hline \multirow[t]{4}{*}{ poiss. (log) } & 124586.0 & 610 & 685.8 & 608 & 123 & 900.2 & $<0.0$ \\
\hline & Covariate & Est. & & se & $z$ & $p$ & \\
\hline & $\log (l)$ & 0.31897 & 0.0 & 1093 & 29.2 & $<0.0$ & 01 \\
\hline & $\log (l)^{2}$ & 0.02443 & 30.0 & 0143 & 17.0 & $<0.0$ & 01 \\
\hline
\end{tabular}


Figure 4.2 shows the correlation of main stem length and minor stem length based on the ProLoc data from the fourth trial year. As expected, there is a positive, linear correlation and the variance of minor stem length increases with main stem length.

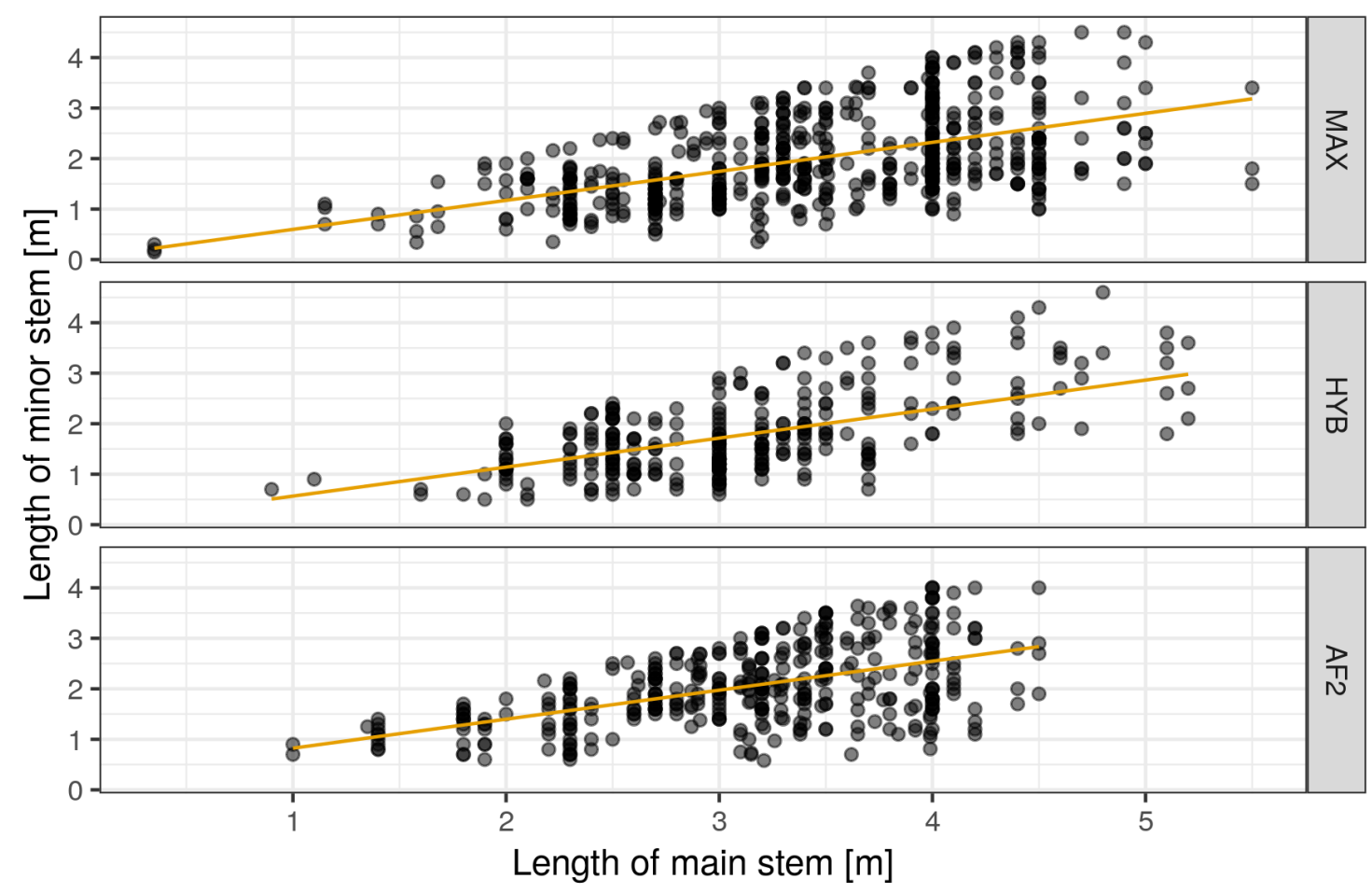

Figure 4.2: Length of minor(s) stem by length of the main stem of the same tree in year 4 separated by clone. The values pairs are from the ProLoc dataset. The yellow lines resemble the predicted values from the linear model fit using gls.

A linear model was fitted using gls with a variance power function to accommodate for the increasing variance. Again, the coefficient from intercept tested as being not significantly different from 0 therefore it was dropped from the model. Besides the length of the main stem lMax the genotype influence was identified as having a significant influence on the response. The differences of 'AF2' with the other two clones indicated by a positive shift of the function line should be pointed out. The predictive power of the model is intermediate as pointed out by $\operatorname{cor}^{2}(\hat{y}, y)=0.32$. The model summary is given in Table 4.2 . 
Table 4.2: Summary of linear model fit using gls for estimating the length of minor stems in year 4. Independent variables are the length of the main stem lMax and the clonal affiliation clone.

\begin{tabular}{cccrcrccr}
\hline$\hat{\sigma}$ & $\hat{\delta}$ & $l l_{\text {Null }}$ & $d f_{\text {Null }}$ & $l l_{\text {Model }}$ & $d f_{\text {Model }}$ & $L R T$ & $p$ & $\operatorname{cor}^{2}(\hat{y}, y)$ \\
\hline 0.33 & 1.04 & -1545.6 & 3 & -1226.5 & 6 & 638.2 & $<0.001$ & 0.32 \\
\hline
\end{tabular}

\begin{tabular}{lrcrr}
\hline Covariate & \multicolumn{1}{c}{ Est. } & se & \multicolumn{1}{c}{$t$} & \multicolumn{1}{c}{$p$} \\
\hline lMax & 0.57445 & 0.01360 & 42.2 & $<0.001$ \\
cloneMAX & 0.02372 & 0.03709 & 0.6 & 0.523 \\
cloneHYB & -0.00821 & 0.04808 & -0.2 & 0.864 \\
cloneAF2 & 0.25009 & 0.04956 & 5.0 & $<0.001$ \\
\hline
\end{tabular}

Since this only describes the growth relation of main to minor stems in year 4, the question remains how to estimate height increment in the fifth and sixth year. Problematic in that regard was that the stems and their height were not matched between years in ProLoc. The main stem was simply identified by having the maximum height value. A tryout was made to sort the minor stems and then calculate the height increment based on rank. This did not yield satisfying results. The decision was made to relate height of minor stems to main stem height in the fourth year and then to use the growth function of the structural model which mainly relies on $\mathrm{GU}$ length of the prior year.

\subsection{Implementation}

With the statistical models parameterized that are needed to bridge the gap between yield and structural model the interconnection itself can be implemented. An overview on how this was realized is given in Figure 4.3 . 


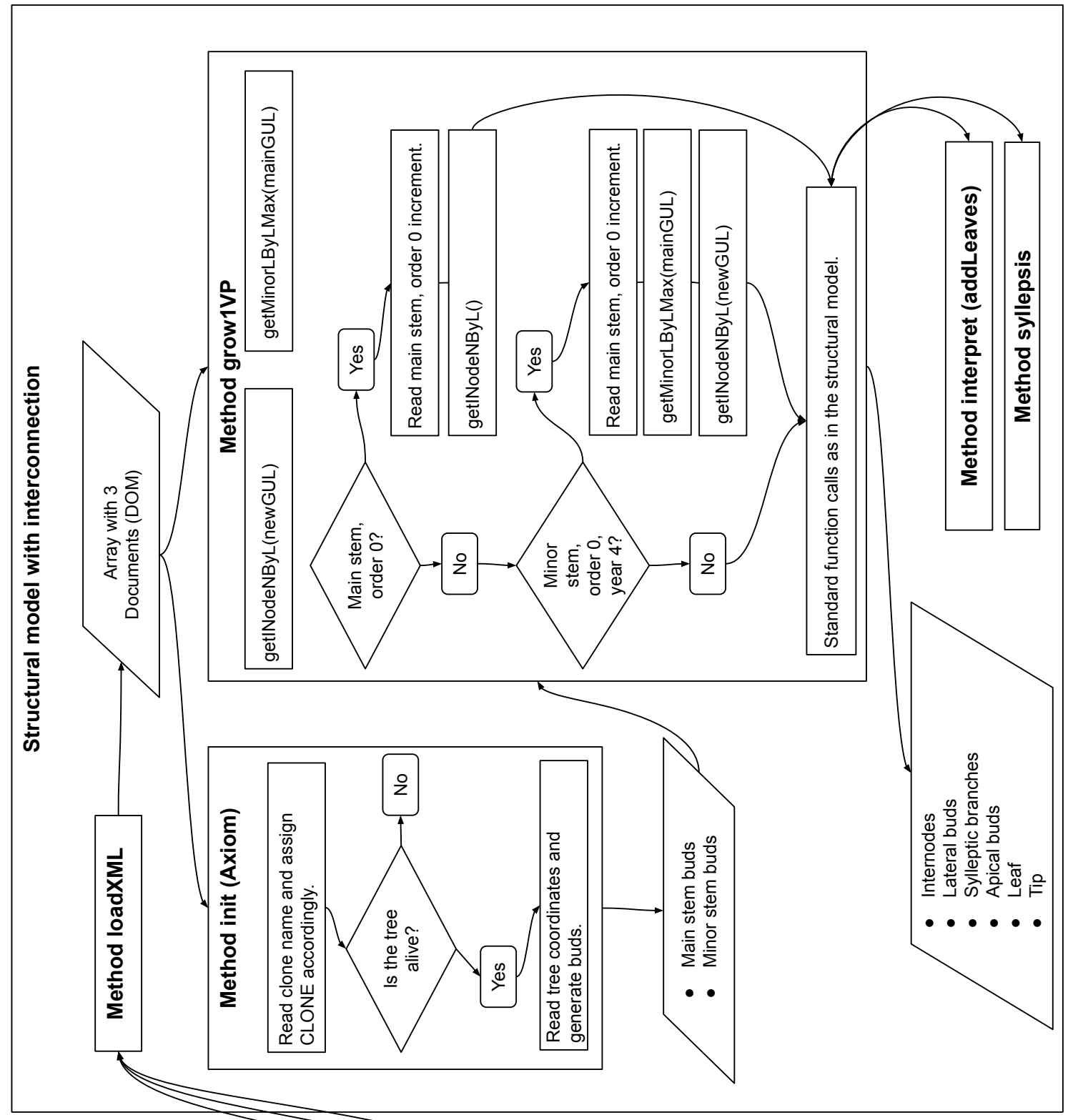

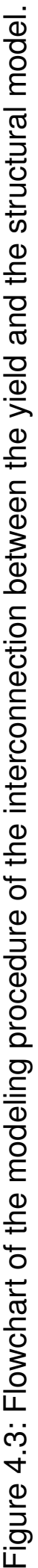


In the left column the yield simulator, the manual input that has to be made for its execution and the output in form of the XML files can be seen. Only the $\mathrm{XML}$ files from the second rotation are shown here, since the first rotation data is not used here. It was decided to implement the interconnection itself within the structural model because this necessitates only minor changes in comparison to other solutions. To make the increment data available that is stored within the XML files, these are loaded through the loadXML method and parsed into a DOM object with a Document interface each. These are then organized by year in an array. There are two cases where these Document interfaces are queried: within the init and the grow1VP method. In both cases a NodeList interface is created from a Document and the Element interfaces in the respective NodeList are selected by their XML tag name. The content of these Element interfaces can then be written to variables for further processing. This is first done in the init method by selecting all Elements with the tag name "Baum" (tree) and all elements with the tag name "Baumartencode" (tree code). The former relates to single tree information and the latter is general stand information. The clone name, which was written by the yield simulator to the XML file, is assigned to a String variable. Based on the content the public integer variable CLONE within the structural model is set. A for loop then iterates through all "Baum" Elements in the corresponding NodeList. The content of the Elements containing the year of death and $x$ and $y$ coordinates are written to separate variables. If a tree is still alive, the GrolMP Trans late function is called using the $x$ and $y$ coordinates. The main and minor stem buds, their number and angles are estimated with the methods as described for the structural model. The treeID of each bud is set according to the index of the "Baum" Element within the NodeList. If a tree is dead, the procedure is skipped to the next iteration step of the for loop. Through this procedure all trees marked as alive in the XML file are generated. Since the CLONE variable and the spacing are now determined by the XML files' content, no manual input is necessary for the structural model.

Within the grow1VP method, the height increment of the main stem is read from the NodeList with the "Baum" Elements. Within each year this height increment is set as the length of GUs from the main stem with branch order 0 . The internode count is then estimated with the model described in Table 4.1. For minor stems, the main stem increment is used as an input for estimating the mean mi- 
nor stem GU length but only in year 4 (see Table 4.2). The mean internode count is then estimated and via a Poisson random number generator the length count of internodes is determined. The other procedures of the structural model remain unchanged and are applied as described in Section 3.2.11.

In Figure 4.4 the graphical output of the structural model with input data from the yield model is shown. The point in time is at the end of the second rotation in year 6 . In the left panel a lower yielding stand was simulated using the environmental variables from location pl12 Kummerow. The right panel shows a stand that was simulated based on the variables from location pl17 Unterrieden. In both cases the growth and survival of 12 trees were simulated. The differences in survival are clearly visible, on the left 4 trees survived whereas on the right 11 trees are still alive. The differences in height are also distinctive, the length of the minor stems is scaled accordingly.

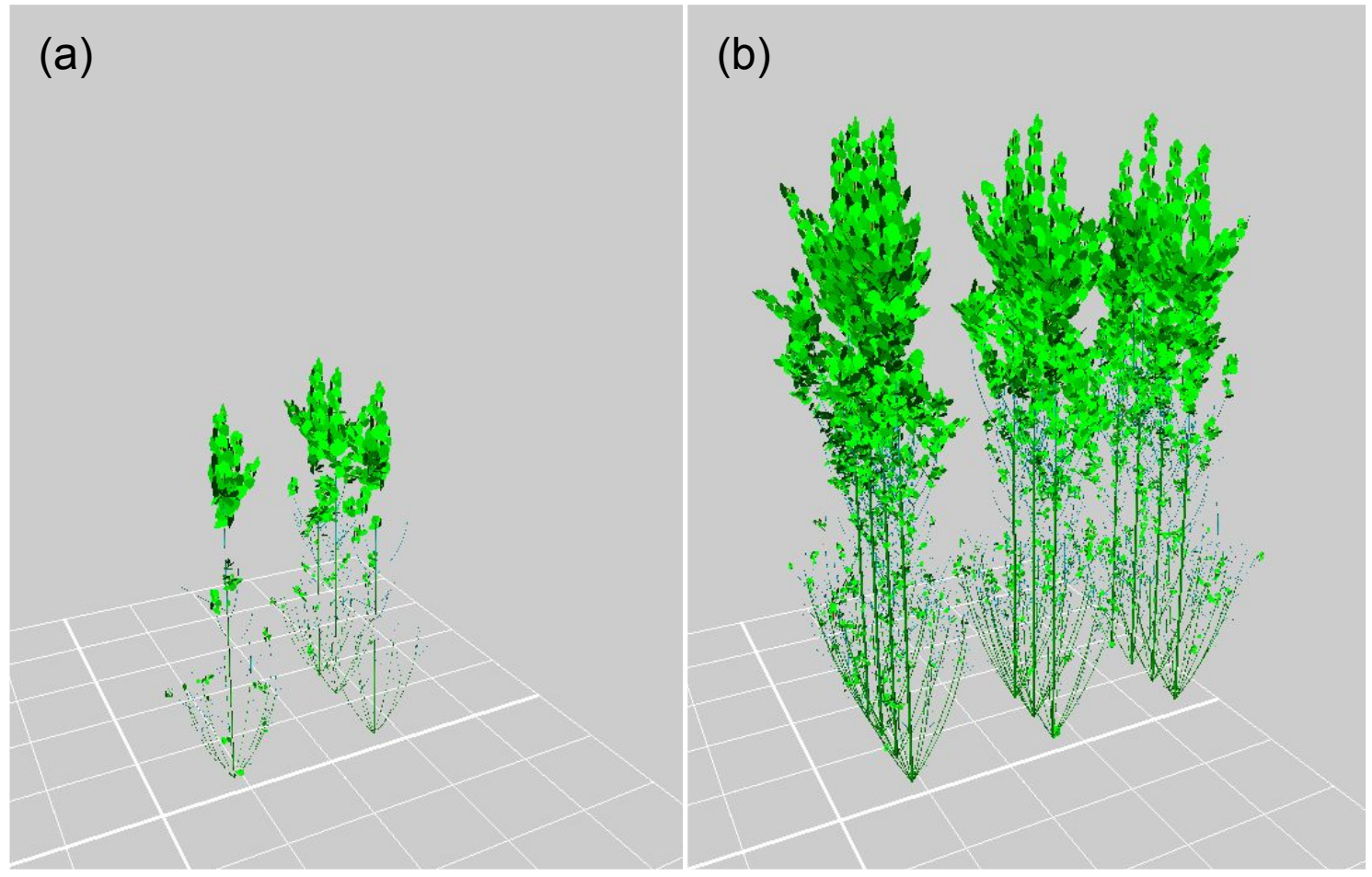

Figure 4.4: Comparison of the 3D visualization of 2 imported stand increment datasets. 


\section{Discussion}

In the following section the results of the yield model, the structural model and their interconnection will be discussed and critically evaluated in the context of other research. While the materials, methods and results were presented separately, the discussion is presented as one section although the order is the same as before.

The overall quality of the data for the yield modeling is satisfactory. The 6 missing year-location combinations (see Table 2.7) are unfortunate though. Especially the missing data in year 4 for four sites can be seen as a bottleneck. It is further disadvantageous that the two locations pl15 and pl25 are missing here but this could not be prevented due to the uncertainties between project phases. An imputation of the values via interpolation procedures (for an overview on $\mathrm{R}$ implementations see Robinson and Hamann, 2011; Moritz and Bartz-Beielstein, 2017) was not considered here but could still be realized for further analysis. Apart from location-year combinations some trial sites and trial-clone combinations had to be completely dropped from the analysis. This is unfortunate in cases of stands with weed pressure or poor planting material quality, but the produced results can not be seen as representative for the environmental conditions that were tested. Janßen et al. (2017) have already proven that the affected sites and plots could recover to some degree from these problems but were still comparably less vigorous.

Moving from the stand and plot level to the single tree scale, matching observations between years turned out to be difficult due to the switching to neighboring trees if the tree within the grid had died. Clearly implausible observations, like large negative increment or trees being alive after marked as dead, could be eliminated from the dataset but there still remains some doubt about other remaining variables. It should be mentioned though, from own experience, that the identification of trees by their ID and plot position can be difficult with a dense 
spacing and $0.5 \mathrm{~m}$ distance within the rows. Another aspect that still has room for improvement, although this was no omission but rather not a project aim from the start, is that the observations of stems per tree could be matched between years (matchable repeated measurements for single stems). Due to not factoring this into the measuring methodology, the within tree competition between stems, especially in the second rotation, cannot be examined too closely. Modeling the growth of single stems is hence left to the structural model and the interconnection. The abstraction of total tree height (as length of the tallest stem) and tree-wise basal area (as sum of all single stem basal areas) could be modeled satisfactorily and might serve the purpose of yield estimation well enough. This eventually facilitates the modeling procedure because another scale (within tree) is left out of the model and with it another source of possible variation.

Assessing the statistical methodology showed that the chosen model types seem well suited for the tasks at hand and their demands. Regarding the data completion, the Näslund/Petterson function performed well in approximating the diameter height relationship. This confirms the finding by Hartmann (2010), at least for poplar. Skibbe (2016) suggests using the Prodan function for the same purpose with willow. For the ProLoc dataset the Näslund function performed equally well for poplar and willow therefore the decision was made to stick to this one function.

Regarding the estimation of the Weibull shape and scale parameters, the differences in predictive power are similar to those already described by Nagel and Biging (1995) although the fitting was done for diameter distributions in their case. The model fitted for estimating the shape parameter possesses a lower predictive power than the one for estimating the scale parameter, but a $R_{a d j}^{2}=0.75$ for the former is still seen as good. The coefficients of determination described by Nagel and Biging (1995) for the shape parameter range from 0.58 to 0.9. Nagel and Biging (1995) additionally list lower values from previous studies from 0.16 to 0.54 .

Using the GLM approach for the survival probabilities is more sensitive than modeling transformed survival rates with linear models (Warton and Hui, 2011). For both models, survival after planting and survival after resprout, the predictive power is however unsatisfactorily poor. For the survival after planting the estimation for the dispersion parameter is also comparably high. Obviously, there are 
other parameters and sources of variations that could improve the model performance. The different site preparation measures and the weeds height were not included into the modeling process as independent variables. Including these could improve the fit since they have a proven influence on survival rates and/or growth after planting (Buhler et al., 1998; Bärwolff et al., 2012; Albertsson et al., 2014). Problematic for the quasibinomial model family on a statistical level is also that the design is quite unbalanced here (Faraway, 2016). No further measures were taken to evade these problems.

Using mixed-effects methodology could compensate for the problems of an unbalanced design (Pinheiro and Bates, 2000). This points towards the general debate if incorporating the location factor as a random effect could be a valid statistical approach. In forest growth modeling, mixed-effects models have been increasingly applied to represent different spatial and longitudinal scales (see for example Nothdurft, 2007; Schmidt et al., 2011; Schoneberg, 2017; Schmidt et al., 2018). Robinson and Hamann (2011) assess that the requirement for an effect to be considered random its allocation/sampling must also be fully random is too strict. On the other hand, their pragmatic approach is to see an effect as fixed if within the analysis the effect is conditioned on. The latter is the case for the ProLoc approach where the environmental conditions, which are subsumed under the location factor, are of central interest for the modeling procedure. Together with the selection process of the sites before the trial establishment the purely fixed effects models seem feasible here.

The variable selection process for these fixed effects is prone to the usual problems when using automated variable selection procedures (Faraway, 2015). The presented models were obtained by carefully checking the plausibility of the results of the variable selection and putting an emphasis on model simplicity. The details of the selection procedure are not presented here since this would extend the scope of this work. The results of the selection procedure should be discussed however.

Within the environmental data and especially the soil data the focus was placed on variables that have already proven useful in prior research on yield modeling in SRC. Both the AWC and the BZ have shown that they both exert considerable influence on growth. The choice of the calculation methodology of the AWC was based on the recommendation by Renger et al. (2009) which implied choos- 
ing the procedure that estimates the AWC most precisely and not choosing the procedure that produces the best correlation with growth parameters. The latter was however the case which points towards the reliability of the method by Renger et al. (2009). The AWC and the BZ were used exclusively within the variable selection because they are quite closely correlated (Pearson correlation $r$ $=0.58$ ). Switching between the AWC and BZ between rotations might appear a bit like overfitting the models to the data by simply choosing the variables that produced the best results without questioning the causation. The fact, that the AWC performed less well as a predictor in the second rotation, points towards the problem that the depth of soil probing to $60 \mathrm{~cm}$ might cut too short for the growth of 4 years and older poplar and willow trees. Both tree species and their hybrids tend to develop the major quantity of roots in the upper soil layer while single roots can reach to greater depths depending on the soil and water availability. For non coppiced trees of species Populus $\times$ canadensis (also referred to as Populus $\times$ euramericana, naturally occurring hybrids between Populus deltoides and Populus nigra), Populus nigra, Salix caprea and Salix cinerea, Kutschera and Lichtenegger (2013) describe that the large quantity of roots develop in the upper soil layer while single roots can reach greater depths of up to $3 \mathrm{~m}$. Hoffmann (1966) used the poplar clone 'I 214' (Populus deltoides $\times$ Populus nigra) in a study comparing rooting depths. Planted as $16.5 \mathrm{~cm}$ cuttings the roots reached depths of over $1 \mathrm{~m}$ already during the first vegetation period in an experiment in a controlled environment. Within the experimental setup a soil mixture, comprised of $83 \%$ sand, $9 \%$ silt and $8 \%$ loam, was used. A decrease of root diameter with increasing depth was observed. This was also confirmed by Crow and Houston (2004). A quantity of 75 to $95 \%$ of roots was found within the Ap horizon for all soil types. The maximum depths that were reached were around $1.3 \mathrm{~m}$ with coppiced stand including trees with stool ages up to 10 years and stem ages up to 7 years. Wittich (1951) also points out that poplars can develop roots to a depth of $2 \mathrm{~m}$ if groundwater is available there. Heyn and Wachendorf (2012) note that not considering the effective rooting depth could misjudge the water supply through capillary rise. They included these factors in their analysis of some ProLoc sites which relied on probing to greater depths adding the layer of 60 to $90 \mathrm{~cm}$. The water balance also has been surveyed to a greater detail than it was the case for all ProLoc sites. This also included the information on hydromorphic soils which 
can be used to further refine the AWC calculation by Renger et al. (2009). As said, this information was available for a subset of 20 sites of which 9 were not part of the here selected subset for the yield model. Furthermore, some testing attempts which included a stand yield model with the total biomass yield as the response and the AWC values by Heyn and Wachendorf (2012) as an independent variable did not improve the predictive power sufficiently in comparison to the variables that were used here. However, this could still be tested within the growth functions here. Further potential exists by incorporating the data from the field trials of the FastWOOD breeding program. Major advantages are that the spacing is the same or deviates only to a minor degree, the same rotation length was used and the here utilized clones were used as a control group for assessing the performance of new clones (Fey et al., 2018). So while comparability is ensured the FastWOOD soil surveying was also carried out to greater depths than $60 \mathrm{~cm}$ (Fey et al., 2018). The further steps here could be first to validate the yield model that is presented here with the data and/or to expand the existing model by even further broadening the data basis. While the environmental range of the FastWOOD sites is not as large as the one of the ProLoc locations, still interesting cases exist where for example for sites with a low BZ of 34 a groundwater influence is obvious due to the high mean yields over 10 odt $h a^{-1} a^{-1}$ that were reached during the first rotation (Fey et al., 2018).

The post mining sites were left out of the modeling process here, as done by Amthauer Gallardo (2014), due to their special characteristics that makes them difficult to compare to the other sites. Two of the mining sites that were left out here have the lowest total biomass yield after two rotations, two others produced mediocre results. These comparably low yields can be confirmed by other publications (Bungart and Hüttl, 2001, 2004). However, SRC plantations, with an expanded tree species spectrum including e.g. black locust, can be an interesting option for recultivation (Böhm et al., 2011).

The influence of soil chemistry on yield was, after correlation analysis, early left out of the modeling procedure. The topic of nutrient supply within SRC plantations of poplar and willow has been subject of numerous research projects, although the results point into different directions.

Ferm et al. (1989) identified a positive effect of nitrogen fertilization on poplar growth which was confirmed by Alriksson (1997); Labrecque and Teodorescu 
(2001) for SRC willow plantations. Hofmann-Schielle et al. (1999) found a positive influence of nitrogen fertilization for willow while the poplar did not react. A fertilization with nutrients phosphorus, potassium, and magnesium showed no effect. Bilodeau-Gauthier et al. (2011) point out that fertilization with nitrogen and phosphorus fertilizer only had a significant effect on growth of hybrid poplars if the site preparations, in this case on acidic, former forestry sites, were sufficient. Sevel et al. (2014) compared the effect of different types of fertilizer: mineral (NPK) and organic (manure, sewage sludge). The clone 'Tordis' was used within the trial plantation. While the growth increase was significant, it was modest in terms of quantity. Furthermore the effect stagnated with increasing doses of fertilizer. Sevel et al. (2014) suppose that the already high nitrogen storage of the soil was sufficient for growth and that the current status of the soil should be evaluated before considering fertilization in SRC plantations. Quaye and Volk (2013) also tested the application of organic and mineral nitrogen fertilizer on willow plantations after coppicing. The fertilization had no significant effect on growth. Comparable to Sevel et al. (2014) the initial nutrient supply of the soil was assessed as sufficient. Balasus (2014) could also not indicate a significant influence of fertilization on tree growth. Poplar clone 'Max 4' and willow clone 'Inger' did not react to nitrogen application. It should be noted though that trial results might be biased due to the described, heavy weeds problem (Balasus, 2014).

Dimitriou and Mola-Yudego (2017) point out that the effect of fertilization depends on factors like the stand age, the utilized clones and the nutrient balance of the soil. As already indicated by Quaye and Volk (2013) as well as Sevel et al. (2014) the nutrient status of agricultural soils can be seen as sufficient for SRC cultivation. This is also supported by the findings of Boelcke and Kahle (2008).

Against this background, the result, that nutrient storage variables did not improve the predictive power and hence were not a limiting factor for growth, seems feasible.

In opposition to this, climate variables had a significant influence on growth and survival. The data basis in form of the interpolated data from meteorological stations can be evaluated as good, as it could only be improved with direct measurements at the trial sites. This would have been too cost intensive within the project. Using the mean values of monthly aggregations over the annual monthly aggregated values might appear like an unnecessary, deliberate loss of informa- 
tion. Using the annual values of the different time periods within the modeling procedure was tested by, for example, using the sum of precipitation during May and June in 2008 for the estimation of mean stand height after the first vegetation period. Proceeding like this for all models only improved the predictive power of the whole simulator slightly. It was therefore decided to use the mean values for the whole trial duration. Using the annual values appeared prone to overfitting the models to the dataset. The latter would most probably be furthered by allowing for different aggregations for each model. While this might increase the predictive power, the respective model will surely lose general applicability. Furthermore, a main goal was to be parsimonious within variable selection. Considering the time periods that were used for aggregating the climate variables, further evidence is given towards the fact that the early months during the vegetation period are most important for growth of poplar and willow in SRC plantations. Especially for precipitation, this has already been indicated by the yield modeling efforts of Ali (2009) and Amthauer Gallardo (2014). Based on the examinations by Hammes (1983) as well as Lindroth et al. (1994), this has a physiological basis as both indicate, for poplar and willow respectively, that the water use efficiency in regard to biomass production has a peak at the beginning of the vegetation period in May. It should be noted however that both publications indicate a second climax in August which could not be reflected within the yield model here. This does not mean that the results of the variable selection imply that the available water plays a role for plant growth only during May but rather that this time period is central for the growth of the whole vegetation period. Further research into the seasonal variation of water use efficiency of different poplar and willow clones, especially of higher yielding cultivars, would be useful in further addressing the remaining questions. Aggregated climate variables like the aridity index (see Amthauer Gallardo, 2014) were not tested on one hand because the correlation of temperature in June and July and precipitation in May and June was comparably low $(r=0.31)$ and on the other hand because it was desired to directly measure their influence on growth.

Another aspect that could be a promising aim of further research would be to investigate differences in phenology between clones. Phenology has been described to be a major factor in the adaptability of a genotype to the climatic conditions (Keller et al., 2011; Fitchett et al., 2015) which will become increasingly 
important in the course of climate change and global warming (Chuine et al., 2000; Chuine and Beaubien, 2001). Research on phenology of poplar (e.g. Pellis et al., 2004) and salix (e.g. Ghelardini et al., 2014) points towards the good possibilities in linking these traits with genetic data. For the clones that were used here, data on bud burst and leaf senescence is available from the FastWOOD project. The data could be used to analyze the differences between clones and for example calculate dynamic vegetation period lengths according to the methodology of Menzel (1997).

Apart from that, by summarizing the results from the variable selection on soil parameters and climate, the water supply in form of precipitation and water storage capacities of the soil are of key importance for poplar and willow growth. These results underline previous findings by Murach et al. (2008), Ali (2009), Bergante et al. (2010) and Amthauer Gallardo (2014).

Regarding the influence of the clonal factor there are, as shown, some differences within the single models that were parameterized. In respect to one of the core aims of the ProLoc project to identify if a growth-determining interaction exists between the clonal affiliation and environmental variables no significant effects were observed. This was already in part established by Amthauer Gallardo (2014) for the first rotation. The model parameterized by Janßen et al. (2017) separated between the genera in the modeling procedure, the methodology of the latter has to be assessed as unsuitable in retrospect though. The biplot analysis by Stiehm and Hofmann (2018) to examine the genotype-environment interaction already pointed out that there are tendencies towards differences between performance of the single clones on some sites. These were however rather vague and could not be linked to environmental conditions (Stiehm and Hofmann, 2018). It is therefore concluded that for the clones that were used here, under the conditions that they were tested in, no genotype- or genera-wise recommendation on environmental conditions regarding optimized growth can be made.

The two remaining factors that were identified as important are the planting date and the single tree competition.

That the planting date influences the survival of the trees within the first vegetation period has been established by Hansen (1986) with the same effect that later planting is antagonistic to survival rate. Hansen (1986) has however also used a wider range of planting dates reaching into late summer and autumn. Regarding 
tree growth it was also indicated that a too early planting can be disadvantageous for height growth (Hansen, 1986). These findings cannot be further validated here as the range of the planting dates is not too wide for the ProLoc data which is actually desirable in terms of a uniform trial design. For evaluating the reasons on why there are significant differences in growth and survival based on planting date the approach by Zalesny et al. (2004) points out promising aspects by defining threshold values for belowground growing degree days (annual sum of days above a certain temperature) for planting poplar cuttings.

Modeling the within-stand competition between trees, the BAL performed adequately. The use of a distance-independent competition index was necessary due to the experimental design and the fact that no neighboring trees were measured. Within the FastWOOD dataset annual growth parameters are available for neighboring trees. It could be assessed if the growth influencing competition in regard to the directly adjacent trees can be modeled with a higher predictive power. The competition index used by Corona et al. (2002) could be applied here as well. More sophisticated approaches could benefit from the interconnection of the yield and the structural model which will be elaborated later.

Using the height as the length of the tallest stem before cutting instead of the BAL before cutting to estimate the height and survival after cutting seems arbitrary. Choosing the height as an independent variable performed better and produced more plausible results. The underlying principle that size before harvest influences the growth after cutting was already proven for the ProLoc data by Janßen et al. (2017) on the stand level.

The predictive power of the survival models after planting and after resprouting is rather poor. It remains unclear why the mortality could not be modeled more satisfactory with the variables at hand. The tendencies that were detected seem plausible but do not explain much variance. Adding survival models for the mortality within the rotations was tested first but lead to a too drastic decrease in survival rates when applied within the simulation procedure. From a stand dynamics viewpoint it is also ambiguous that trees that would have been determined as dead would be completely kept out the further simulation process until the end of the rotation. While they surely play no role in competition anymore their biomass, even if dead, still remains in the stand and is harvested due to the clear cut regime. That the tree is in fact completely missing, as assumed in the simulator, becomes 
apparent when the stool does not resprout after cutting. Again, the modeling on single tree level could benefit from the interconnection with the structural model by more faithfully approximating the underlying processes of mortality in terms of stand structure and physiology (shading, competition for nutrients).

Summarizing the results from the variables selection and evaluating the overall simulator performance, the results are satisfactory. Compared to the yield models by Ali (2009) and Amthauer Gallardo (2014), the predictive power of the combined models in forms of the growth simulator is lower, especially within the second rotation. It needs to be minded though that the models here were fit to a dataset that contains between environment and within environment variance. The latter includes the stand dynamics, competition and microsite effects as well as the temporal variance. The models by Ali (2009) and Amthauer Gallardo (2014) were fitted on the stand level. Against this background, the lower predictive power seems adequate. Another aspect was that multiple models were parameterized and combined later on which posed the challenge that this can add up the models' prediction error. Setting up the simulation procedure early on in the analysis and, whilst checking the single model fit, also assessing the overall simulator performance, minimized the effect of accumulating bias. The advantage of the single tree based approach towards a stand based model are the insight into the within-stand dynamics, the more precise estimation of single tree size, which can be useful for the decision process related to harvest, and the increased potential for the interconnection with more finely scaled models like the structural model.

The fact that the predictive power is lower for the estimation of MAI in comparison to the mean stand height estimation is based on the error that is produced by the MAl estimation. Based on the experiences made with the biomass estimation approach by Hartmann (2010), the best results can be obtained by estimating single stem biomass based on the $\mathrm{DBH}$. A parameterization was not possible here as the dry mass was measured on the single tree level. The biomass functions that were parameterized for yield trial analysis in FastWOOD (Grotehusmann et al., 2015, 2017) could be of use here although the relationship of the function parameters with the factor location would need to be considered. An also promising approach would be to use the interconnection to calculate the wood volume of stems and branches per tree. Based on the dry wood density values published by Amthauer Gallardo and Seymour (2011), the dry mass could 
be estimated per GU. This would also allow for an assessment of the quantity of fine branch biomass and hence combustion properties (Amthauer Gallardo and Seymour, 2011) for the use of the biomass in producing energy.

The practicability for the landowner to employ the yield model approach presented here can be assessed as good. The simulator is easy to use and the variables that are needed for growth estimation are also easy to acquire. In planning, the date of planting can be fixed beforehand, climate variables can be obtained from public sources or through the DWD. The BZ is a common measure for agricultural land. The AWC can be calculated with the publicly available methods by Renger et al. (2009). The input for the calculations can be manually measured or should be provided based on laboratory analyzed soil probes.

A final caveat should be given regarding the overall model and simulator validity. Although the range of the environmental conditions that the model is based on is quite wide, especially in comparison to prior research, there are still limits to the model and hence restrictions in its application. The model is trivially only valid for the selected clones within the range of the climate and soil conditions and only for a duration of two rotation cycles. An extrapolation of the estimation beyond the range of the observed conditions might produce unreliable predictions which often becomes more problematic for linear models, even with transformations (see Nothdurft, 2007). Furthermore the results only apply for the chosen cultivation technique implying spacing and rotation length. Apart from the possible limits imposed on growth by the environment, the simulation assumes otherwise ideal conditions (Hansen and Nagel, 2014). External biotic stresses like pests, insects, browsing and weed competition are not considered within the procedure. Therefore the model complex cannot be assumed to be generally applicable to any conditions when predicting the yield potential of SRC plantations. On a side note, the simulated stand does also not incorporate any border or margin effects. The trees that are simulated, are, as it was the case for the experimental data, assumed to all be growing within the plantation. This could be of importance when the tree growth in strips of agroforestry systems (see Böhm and Veste, 2018) is simulated.

As stated by Hansen and Nagel (2014) the user should keep these limitations and the scope of applicability in mind while critically scrutinizing the produced results. 
Some of the limitations already point towards further research needs. These include the incorporation of growth dynamics in later rotation cycles, rotation cycles of varying lengths and hence different spacings. Later rotation cycles could be included through the analysis of data from some ProLoc and FastWOOD stands that were harvested after the third rotation. Attempts to examine the factors of longer rotation cycles and varying spacing by initializing further trials sites were made in ProLoc but were limited by the short trial and hence supervision length and by poor site conditions that superimposed the effects of spacing (see Janßen et al., 2017).

Moving to the discussion of the results of the structural model, first the measuring methodology will be examined.

Evaluating the different methods for measuring woody biomass structure, a clear recommendation cannot be made. Choosing the right approach depends on the aim of the study. The manual dtd approach requires only few measurement instruments, it is fast to employ and its requirements to the measurement environment depend on the surveyors mainly (relatively dry conditions for working). The experience has shown that it would be better to digitize the measured values with a laptop in the field and to directly validate the dtd data in GroIMP to spare later plausibility checks.

The semi-automatic approach yields, when compared to the manual approach, more detailed data in the same period of time. Measuring the 3D node coordinates offers more possibilities in further analysis of which the internode length and branch curvature were used here. While working with the FASTRAK device demands only two workers the requirements for the environmental conditions are higher and therefore the planning needs to be more thorough. Regarding the usability, the Blender addon by Wasilczuk (2012) works well. Minor improvements to the sorting of identifiers of measured points within the program and the export format of the 3D coordinates could further ease the handling. The post processing of the FASTRAK data for further analysis demands also more effort than the dtd data but worked quite well once the routines were implemented. On a side note for possible further measurements, the calibration points for joining separately measured parts should be placed with enough distance $(50 \mathrm{~cm})$ along the stem or more points should be added to have a more precise alignment via the Procrustes analysis later on. Otherwise it should be considered to obtain the 
long-range extension for the FASTRAK device like Danjon and Reubens (2008) and Surový et al. (2011) to supersede measuring separated parts. This of course is inherently unnecessary for the manual measuring technique. As the approach here was static by measuring the trees once and reconstructing their past growth, cutting the trees and assuring a proper alignment of separated parts is laborintensive. To measure all parameters on a still standing tree however requires even more resources due to the height after 3 years and more effort would have been needed to plan and build a scaffold for example to ensure an efficient and safe work environment for the surveyors. If measurements are to be conducted with older trees or even with adult trees of other species in forest stands these aspects have to be reconsidered as felling without damaging the structure becomes more difficult.

The semi-automatic approach could also be applied to measuring the leaf position and structure as well as shape. This would also imply that the further analyses can be made due to the availability of 3D point data which would however also increase the demand in post-processing the data. The largest drawback would be the higher demand to wind conditions when measuring. Windy weather can already be problematic for the manual measurement but it will surely lead to increased bias especially when digitizing points on the leaf blades. This could be mitigated by further efforts in planning or by setting up wind shelters. Nevertheless, this was assessed as not feasible with the resources at hand.

Under greenhouse conditions, Kahlen and Stützel (2007) have successfully used the semi-automatic approach to digitize shapes of cucumber leaves. For this, 17 points on the leaf blade were defined that were consecutively measured. By triangulation of the 3D coordinates of these points, the shape of the leaf can be approximated. This has the advantage over the approach chosen here that factors like leaf bending can be examined while here the leaves are assumed to be perfectly flat. Henke et al. (2014) already point towards the possibility to include bending along the midrib in GrolMP using NURBS shapes. For developing their method, Henke et al. (2014) have chosen black poplar leaves based on their flat morphology and to assume the leaves to be flat does not seem like a dramatic simplification in case of the clones used here. Another advantage of the leaf shape digitization by Henke et al. (2014) is that the full contour is available for further processing and even the shape of the leaf edge itself can be modeled (al- 
though this was not done here). The full contour could also be measured with the FASTRAK device by running the stylus along the leaf blade edge in a continuous measuring mode. Although this has not been tried, it is seen as immensely difficult as constantly touching the leaves will surely introduce a considerable bias, even under greenhouse conditions. Elewa (2010) gives an overview on further methods for analyzing and determining leaf shape. As the results, that were obtained here by comparably simple means, are satisfactory, these methods will not be further discussed.

As a summary, the decision to opt for the semi-automatic or manual approach in measuring the structure of woody biomass depends on the scope of the study and the resources at hand but the semi-automatic method seems favorable due to the increased possibilities that the data offers in further analysis. For measuring leaf position and structure these advantages still exist but the planning and implementation of the measurement becomes more labor-intensive. This would be reduced for measurements in a greenhouse environment. The procedure devised by Henke et al. (2014) can be seen as the first choice under both field and greenhouse conditions.

The procedures that were chosen for post-processing of the digitized data performed well. The 3D to 2D projection via the principal component analysis did not result in much loss of information as the first two principal components could explain a large majority of the variance. The post processing of the digitized leaf shapes could easily be implemented due to the already good output format from the plugin by Henke et al. (2014). The further post-processing mainly focused on rotating and re-aligning the $2 \mathrm{D}$ coordinates which did not cause any noteworthy problems.

Focusing on the applied statistical methodology in the structural model, using the Poisson random number generator also for the models parameterized with the negative binomial model family appears unfitting. As described, using the negative binomial random number generator led to predictions outside the range of the observed data. While this must theoretically not be false it still led to problems with some of the models that use the generated values as input. Using the Poisson random number generator was used as a workaround as, within the model parameterization, the log-link function is used for both model families. The negative binomial model family was used here in the first place to better accom- 
modate for the overdispersion that occurred in the count data (Faraway, 2016). An alternative would be to parameterize generalized linear mixed-effects models (see Venables and Ripley, 2010) which could in a first step account for between tree variability by adding the factor tree as a random effect. In contrast to the discussion on the factor location within the yield model, this is feasible here as the sampling of the trees was random. A more sophisticated approach would be however to identify the sources of the overdispersion and include them as independent variables into the model. The between tree variation in internode counts could be accounted for by incorporating the competition within the stand into the structural model. To some degree, this is already realized by the interconnection since the yield model contains the BAL as an independent variable. An integration into the structural model itself would be more sensitive and will be elaborated later. Besides competition, other factors like varying microsite soil conditions contribute to a between tree variation. These are however even harder to measure and accordingly more difficult to tackle.

The stochastic components within the model in form of the random number generators already create some variation in size and therefore the other parameters that are estimated by GU length for example. This variation mimics growth dynamics which already creates the impression of competition between trees although it is completely random. Adding further variance by incorporating the residual standard error with a normal distribution random number generator as done by Casella and Sinoquet (2003) seems unnecessary.

Another aspect of the statistical methodology is that the assumption of independent errors might be violated. Analyzing the topology already implies that there are certain relationships and dependencies of measured values between the GUs as observation units (see Kurth and Anzola Jürgenson, 1997). Additionally, although the number of measured GUs is quite high the sample of trees measured is comparably small (see Kurth and Anzola Jürgenson, 1997). In regard to the latter it is advantageous that clonal material was used but if the between tree competition should be examined more closely a larger sample is needed. Accommodating for correlation between GUs and increasing the sample size would entail however a significant additional expense which was spared here. These aspects should however be minded when scrutinizing the presented results.

As it was also the case for the yield model, the problems that are connected 
to unbalanced data arise here due to different group sizes. This was mitigated to some degree by using the type III sums of squares in testing hypotheses about regression coefficients and comparing models with the analysis of variance function (Fox and Weisberg, 2019).

The 25 models that were fitted for the structural data (excluding the two models that were not used in GrolMP and the 9 models for the contour points) performed overall satisfactorily. The indicated predictive power ranged from $R_{a d j}^{2}=0.04$ to $R_{a d j}^{2}=0.95$ with a mean of 0.52 . The latter value needs to be interpreted with caution though as this mixes the coefficients $R_{a d j}^{2}$ and $D_{a d j}^{2}$. The models that focus on probability produced mainly low to intermediate results, while models focusing on growth parameter relationships like length to diameter produced the best fits, as expected.

The estimation of the contour point coordinates for estimating the leaf shape mostly yielded satisfactory to good results in regard to predictive power of the single models. The $R_{a d j}^{2}$ values ranged from 0.13 to 0.74 with a mean value of 0.54 . Their combined performance can be evaluated as good especially concerning the leaf blade area. The half contour length is exposed to some bias as the leaf margin has not been modeled here which is tolerable.

Comparable to the fitting process for the yield model, it proved to be practical to successively fit the models and directly integrate the parameterized functions in GrolMP. The basis for this was the structural model by Plazas Cebrian (2014) which was changed and extended gradually. The combined assessment of the fitted statistical models and their direct implementation in GrolMP ensured a fluent joining of the separate modules.

As mentioned briefly, the procedure for differentiating main and minor stems based on height and diameter seems arbitrary. While there clearly is usually a single dominant stem in poplar trees after coppicing, the decision procedure here seems rigid. It was a necessary simplification however to make the measuring methodology more practicable and it allowed for an easier interconnection with the yield model in the end as the height growth was here also related to the main stem. The approach could be improved by leaving out the factorial differentiation between main and minor stems by further examining and incorporating the processes that lead to the emergence of the main stem.

Using the grouping for measuring directional angles can be discussed as an 
unfavorable aggregation of a variable that otherwise could be measured continuously in degrees. Data with the latter resolution could be analyzed by means of circular statistics (see Pewsey et al., 2013). Some approaches were pointed out by Danjon and Reubens (2008) but these mainly focus on influence of wind direction on growth parameters. Regarding the grouping of an otherwise continuous variable Stewart (1983) has pointed out some of the statistical problems in analyzing interval data while Faraway (2016) argues that continuous data modeling methods are feasible if the intervals are narrow. Continuous data could still be derived and analyzed from the 3D structural data from the FASTRAK measurements. However, especially for the phyllotaxis the aggregation seems acceptable as the results could be backed by the references of Coxeter (1969) and Kadereit et al. (2014).

Deviations from the divergence angle of $135^{\circ}$ were observed for the arrangement of minor stems on the stool and for leaves within short shoots. These deviations point towards an optimized orientation and distribution of organs in space and are most likely influenced by additional factors like light radiation and hormonal growth regulation (for resprouting see Sennerby-Forsse et al., 1992).

Regarding the internode length the approximation within the model seems satisfactory. Casella and Sinoquet (2003) also describe that internode lengths are shorter at distal and proximal ends while being longer in the middle of a shoot.

Modeling the diameter increment relies on the mean annual increment here which of course is a simplification. Improving this approach would necessitate to have either further feedback from the change in diameter to length from the yield model (which could only be realized for the GUs that developed in the first year with branch order 0 ), to undertake measurements of tree discs by counting tree rings (see Henke and Sloboda, 2014) or to approximate growth dynamics by repeated measurements. In the given order, the different approaches are increasingly more sophisticated and allow for deeper insights but are also increasingly more labor intensive.

Next to the mean increment simplification the geometrical representation of GUs within the model can still be improved. Currently, cylinders are used which also only resemble the mid diameter and omit any taper that is present within stems and branches. This could either be improved by calculating the mean decrease in diameter from the GU base to its distal end or by additionally measur- 
ing the diameter for each internode when conducting the semi-automatic measurements. Instead of cylinders, frustums could then be used, comparable to Casella and Sinoquet (2003), to represent the internodes within the structural model. This would still neglect however that the woody biomass of the internodes is not shaped perfectly straight. This curvature within internodes can either be approximated by composing a single internode of several frustums for example or by representing it with NURBS objects. Due to the observed length of the internodes the curvature within the elementary unit of modeling seems negligible.

On a general note, the chosen procedure to model the branch curvature is purely empirical and does not take the underlying processes for branch bending into account. More sensitive would be to aim at the biomechanical foundations for branch curvature and bending. Prior research efforts have focused on mechanically modeling the shoot as an elastic rod (Jirasek et al., 2000; O'Reilly and Tresierras, 2011; Guillon et al., 2012). Bastien et al. (2013) has also elaborated on the hormonal influence on bending which regulates cell elongation. To implement a bending based on gravity, tropisms and hormonal balance would however implicate a much greater effort in modeling and sensitivity analysis which is beyond the scope of this thesis.

The option to use the tropism functionality in GrolMP, as done by Plazas Cebrian (2014), and to check which parameter approximates the curvature best, was considered first but then discarded as this would have necessitated some optimization efforts between the GrolMP output and the statistical model. Directly modeling the curvature angles seemed more sensitive.

Despite the potential for further enhancements of simulating the branch curvature the current results are satisfactory as they reproduce the differences in tree habitus between the clones quite well, especially, as mentioned, the more slender habitus of 'AF2'. 
The general branchiness is highest on 'Max 1'. For the two other clones the differences by main and minor stems and by shoot age need to be considered though. The higher probability of lateral shoots to occur and their increased length towards the mother GU's distal end resemble the branching type described as acrotony (Hallé et al., 1978; Roloff, 2001) which is common for Populus (Bartels, 1987). The probability for short shoots to occur is generally higher for 'Hybride 275' although 'AF2' has the highest probability for short shoots on its main stem. The observation that short shoots mainly occur in the middle part of the crown, while long shoots develop in the upper part, can be confirmed by Dickmann et al. (2002).

With increasing branch order and decreasing age, a decrease in internode count and hence GU length can be observed. Although the factor age is not included within the model, a decrease in internode count with progressing years is inherent to the estimation and can be confirmed from the modeled trees (see Figure 3.59). This effect has already been observed in the yield model with a negative coefficient for the covariate year within the model for estimating height increment in the second rotation. These dynamics are in part probably based on the resprouting physiology. After the above-ground biomass has been harvested and as the stool remains in the ground, the roots can abundantly supply reserves to just developing shoots and the transport distances to the developing leaves are comparably short (Sennerby-Forsse et al., 1992).

The transition from preformed to neoformed growth was not considered in the model although it is a central factor in the shoot development of poplar (Bartels, 1987). The transition could not be determined within the structural measurement as there were no observable differences on the shoots in winter condition. The attempts that were made to estimate the point of transition based on the leaf morphology measurements (see Critchfield, 1960; Bartels, 1987) did not yield a clear differentiation. Dynamic measurements, that are repeated over the course of the vegetation period and monitor the growth more closely, could however allow for examining this phenomenon.

The importance of sylleptic shoots for growth vigor has been described by $\mathrm{Wu}$ and Hinckley (2001); Broeckx et al. (2012) although there seems to be an interaction with environmental conditions (see Marron et al., 2006). The probability for sylleptic branches to occur was not too high for the observed clones. This aspect 
seems hard to examine in retrospect though, as smaller sylleptic branches have a higher probability to abscise (Wu and Hinckley, 2001).

The fact, that the possibility of sylleptic GUs having any sylleptic daughter GUs of higher branch order had to be ruled out, was mainly attributed to modeling problems that would have occurred otherwise. The reason for this was using the covariate order by which the model is limited to the observed branch orders. It would be more sensitive to model the occurrence of sylleptic branches based on genetic properties and more importantly on underlying physiological processes. Regarding the latter, Cline and Dong-IL (2002) have pointed out the possible hormonal influences on this phenomenon.

Marron et al. (2005), Verlinden et al. (2013) and Gebauer et al. (2016) have outlined the importance of leaf traits and their variance with genotype as a determinant of yield in poplar while Marron et al. (2007) have pointed towards the possible interaction of leaf related traits and environment.

Regarding the influence of resprouting, Ceulemans et al. (1996) have elaborated on the aspects of leaf morphology and physiology. Sennerby-Forsse et al. (1992) have also pointed out the differences in leaf morphology between leaves growing on shoots that have developed from cuttings or, respectively, after coppicing. There is hence a further need for validation, as by now, the leaf growth in the first and second year of the rotation cycle is simulated based on models parameterized with data from the third year. This could not be mitigated due to the lack of data from prior years.

Apart from that, Dickmann et al. (2002) confirm the observation that leaves on long shoots in the upper part of the crown tend to be larger while the leaves in short shoots in the middle and lower section of the tree are smaller. Al Afas et al. (2005) also support the observed gradient in leaf size. Their observations also back the size transition in the canopy regarding petiole size and hence its correlation with leaf size. Regarding this aspect, the parentage of the clones is also of interest as 'Hybride 275' has comparably large and longer leaves with shorter and thicker petioles while 'AF2' has shorter broader leaves which are positioned by long, slim petioles. 'Max 1' inherits an intermediate role in these leaf characteristics. As already mentioned, this resembles the different sections that were used in cross-breeding these hybrids. Al Afas et al. (2007) observed the same relations in leaf size between intra- and inter-specific clones of the sections 
Aigeiros and Tacamahaca. The leaf shapes also display these genotypic relations which can be confirmed from the taxonomic section descriptions by Dickmann and Kuzovkina (2014).

As already pointed out for the yield model, the model validity and its limits need to be stressed. The results from the structural simulations are limited to the 3 utilized clones, grown in the described spacing within the 3 years of the second rotation under the environmental conditions of the supervised trial sites. Using this model or its output must always be reflected upon critically.

The overall model quality has, apart from the fitting of the single models, been mainly assessed visually which led to satisfying results. Of course, more validation effort is needed here. A first step could be to compare summary parameters like branchiness or branch dimensions in groups calculated from the model with the same parameters calculated for the fully measured trees. An alternative would be to make leaf area index ( $\mathrm{LAl}$ ) measurements or take fish-eye photographs within the existing plantations and compare these to calculated LAI values or re-creations of the photographs in simulated stands as done by Casella and Sinoquet (2003).

Another aspect that is currently not integrated into the model is collision avoiding. This means that the tree organs may grow through other organs like GUs through other GUs or leaves through GUs and so forth. Jirasek et al. (2000) have devised a mechanism for elements of structural models to accommodate for the physical forces that colliding objects impose on each other.

The reconstruction of growth dynamics in prior years based on data obtained in the third year implies a simulation of dynamic processes based on static observations. The shortcomings of the leaf morphology modeling in that regard have already been mentioned. Furthermore, the combination of structural observations, made after the vegetation period with leaf morphology data acquisition conducted within the vegetation period, results in a somewhat artificial constellation. Other aspects like the diameter increment can only be reconstructed in its average form. The same applies for wilting and senescence of GUs whose progression can hardly be reconstructed based on static measurements. Other parameters like branching angle are assumed to not underlie any changes with growth. Especially for the latter, approaches that focus more on the underlying mechanistic principles could more faithfully simulate the tree architecture. 
A further extension of the model could be focused on integrating the first rotation cycle to fully grasp the morphological changes that are induced by coppicing. A model that is able to represent the tree-like morphology in the first rotation could also be extended for longer rotation cycles and wider spacings. In that regard it would be feasible to integrate a sensitivity in shoot growth to flexibly simulate the influence of growing space on tree architecture. This could be useful for optimizing spacing in regard to yield potential as an empirical approach using spacing trials is exceedingly labor and cost intensive. The also existing need to apprehend the environmental influence and its interaction with spacing regarding tree architecture makes the modeling based on yield trial data increasingly difficult (see Janßen et al., 2017).

An examination of the structural development of willow would be of interest as well, especially concerning the already mentioned differing multi stem morphology after coppicing. Regarding the genotypic influence on structure it would be however more feasible to include further poplar clones into the modeling procedure. Especially for the newly crossed, higher yielding clones from the FastWOOD breeding program (see Fey et al., 2018) a higher variety in structural properties is to be expected. Furthermore, the physiological component should be examined. The implementation of a fast phenotyping procedure under greenhouse conditions could aid the breeding process in fast selecting promising genetic material. The benefits of greenhouse trials with a focus on poplar have been pointed out by Krabel et al. (2018).

As such experiments should focus on physiology in addition to structure, the extension of the current structural model to a full FSPM is indicated. Plazas Cebrian (2014) has already pointed out the possibility of such an extension. The modular composition of the presented structural model using separate functions for the separate growth processes should provide proper possibilities for adding more advanced, sophisticated components. In the case of the mentioned greenhouse experiments the existing approaches by Rauscher et al. (1990) and Buck-Sorlin et al. (2005) could be modified for younger poplar trees. Both the SECRETS, as adapted by Deckmyn et al. (2004), and the 3-PG process model, with expansion by Headlee et al. (2013), contain promising approaches to simulate physiological processes in older plantations. For the 3-PG model, Sands (2004b) has already outlined the parameters that need to be provided to adapt the model to new 
species. Common to the mentioned process models is that the central aspects for the physiological processes are mostly light radiation, temperature, water and nutrient availability, respiration, photosynthesis and the allocation of assimilates. The models by Deckmyn et al. (2004) and Headlee et al. (2013) would require some adaptations to the within tree level and the scale of tree organs. Furthermore, a change in temporal scale (away from 1 year growth steps) of the current structural model is necessary and the interdependence of structural and physiological properties would entail some validation effort to obtain simulation results and visualizations that approximate the actual tree growth well enough. Via the interconnection or an aggregation of the results, the model could be used to simulate the influence of different environmental conditions. The precision of the FSPM could then be tested against the large basis of yield data from the ProLoc trials. This aim is comparable to the motivation Deckmyn et al. (2004) and Headlee et al. (2013) who have employed the process-based approach to mitigate the shortcomings of yield modeling.

The implementation of the interconnection of the yield model and the structural model was successful. The focus was here on finding a technical solution to pass data from the yield model to the structural model and utilize the information in simulating the tree architecture. As a short note on this solution, using the XML files with the BWinPro format and parsing the contained information into DOM objects has proven to be sufficient and easy to adapt for the requirements here.

Other known interconnections for GrolMP with forest growth models have been implemented by Schön (2014) for SIBYLA and by (Oberländer, 2019) for BWinPro. Schön (2014) is using the dbh, tree height, both their increments as well as crown diameter and crown base as output variables from SIBYLA which are then passed to a structural model for Norway Spruce. The latter is an adaption of the model by Kurth (1999) with newly measured data. The interconnection itself is implemented through a relational database which is also used by SIBYLA. The needed parameters for the structural model are estimated based on additional model functions (e.g. branch length is estimated based on tree height increment). (Oberländer, 2019) uses the XML files that are output by BWinPro to generate XL code whose interpretation then visualizes the tree structure in GrolMP. Both approaches were considered for the implementation of the interconnection here but were not further utilized as a solution was needed that would fit best to the already 
existing yield and structural model.

With fitting only two models, the adaptations that needed to be made to utilize the yield model output within the structural model are quite minor. Hence, the implementation can be seen as parsimonious. The predictive power of the first model to estimate the internode count based on the height increment (as GU length) from the yield model is very high, as expected. The second model for estimating the minor stem length based on the main stem length has an intermediate predictive power. There is still considerable variance left which most probably could be explained by further scrutinizing the within tree competition between stems after coppicing. As described, the minor stem growth could not be adequately modeled based on the yield data therefore the growth function already implemented in the structural model had to be applied.

The main restriction for using the interconnection is computational power as it transpired that importing large stands into the structural model noticeably slowed down the XML parsing as well as the simulation of the stand itself. By trial and error, it was determined that a stand including 12 trees can still be imported without reaching the limits of the utilized desktop PC. Solutions to this problem could be either to consider an optimization of the parsing procedure as well as the structural model code concerning performance or to employ more powerful hardware. There were no further efforts made regarding these aspects as the simulation of 12 trees seemed sufficient for now.

The visual assessment of the results of the interconnection is satisfactory. The structural proportions of smaller trees on poorer sites, as shown in Figure 4.4 . appear fitting.

As the current implementation of the interconnection is quite basic concerning the exchange of information, some possible improvements were already highlighted in this Section. An extension, that would be comparably easy to implement, is the calculation of dry mass through the dry wood density (taken from Amthauer Gallardo and Seymour, 2011) based on the GU volume. For the latter, the volume of the cylinder objects could be used to calculate the dry mass of each GU and, through a query, the whole dry mass per tree could be calculated. The dry mass could further be grouped by length and diameter of the GUs to allow for an assessment of the quantity of coarse and fine woody biomass.

Another extension should be made by implementing the exchange of data from 
the structural model back to the yield model. Based on the structural model, other competition indices could be calculated. The distance independent version of the C66 competition index (as used by Hansen and Nagel (2014) in BWinPro) could be calculated based on crown parameters which could be aggregated for each tree within the structural model. The procedure would be to estimate the growth after planting or after resprouting within the yield model, followed by passing the individual tree heights to the structural model where the competition index is then calculated. The index value is then passed back to the yield model for the growth estimation in the following year. This procedure is then repeated until the end of the rotation cycle.

As it was outlined for other aspects, it would be more sophisticated to aim at the underlying principles of competition between trees for growing space. A full FSPM could prove versatile in estimating single tree growth potential by competition for light radiation for example. If nutrient and water availability as well as temperature were to be integrated into the FSPM, the environmental factor could also be incorporated. The modeling of tree growth as influenced by competition and environment then rather becomes a question on how to fully implement this on part of an FSPM and then aggregating its results on the stand level to provide parameters which would be of interest in research and management.

Concluding the discussion of the acquired results, the aims that were defined at the beginning of this thesis in Section 1.4 have been fulfilled. As pointed out, promising research questions remain open which should be tackled in the future. 


\section{Appendix}

The source code of the implemented yield simulator, the structural model and the interconnection is made available through the author's GitHub account. This offers the possibility for version control hence tracking future changes will be inherently documented. Furthermore, choosing this platform enables other users to clone the source files and make their own changes if needed and also contribute them to the repositories. Two repositories were created to separate the pure Java implementation of the yield simulator from the $\mathrm{XL}$ based structural model and the interconnection.

The source code for the yield simulator with all needed settings files can be obtained through the following address.

https://github.com/CBSti/Yield-Model-SRC

The source code for both the structural model and the interconnection with some sample XML files exported from the yield simulator can be found in the following repository:

https://github.com/CBSti/Structural-Model-Poplar

Additionally, the reader or further users are encouraged to contact the author of this thesis as the maintainer of the repositories via GitHub if questions arise or suggestions for improvements want to be made. 


\section{Bibliography}

Ad-Hoc-Arbeitsgruppe Boden der Staatlichen Geologischen Dienste und der Bundesanstalt für Geowissenschaften und Rohstoffe and Sponagel, H., editors. Bodenkundliche Kartieranleitung: mit 103 Tabellen und 31 Listen. Schweizerbart, Stuttgart, 5., verb. und erw. aufl edition, 2005. ISBN 3-510-95920-5 3510-95920-5 978-3-510-95920-4.

Al Afas, N., Pellis, A., Niinemets, U., and Ceulemans, R. Growth and production of a short rotation coppice culture of poplar. II. Clonal and year-to-year differences in leaf and petiole characteristics and stand leaf area index. Biomass and Bioenergy, 28(6):536-547, 2005. ISSN 09619534. doi: 10.1016/j.biombioe. 2004.11.010.

Al Afas, N., Marron, N., and Ceulemans, R. Variability in Populus leaf anatomy and morphology in relation to canopy position, biomass production, and varietal taxon. Annals of Forest Science, 64(5):521-532, July 2007. ISSN 1286-4560, 1297-966X. doi: 10.1051/forest:2007029. URL http://www.afs-journal.org/ 10.1051 /f orest : 2007029 .

Albertsson, J., Hansson, D., Bertholdsson, N.-O., and Åhman, I. Site-related set-back by weeds on the establishment of 12 biomass willow clones. Weed Research, 54(4):398-407, August 2014. ISSN 00431737. doi: 10.1111/wre. 12086. URL http://doi.wiley.com/10.1111/wre.12086.

Ali, W. Modelling of Biomass Production Potential of Poplar in Short Rotation Plantations on Agricultural Lands of Saxony, Germany. Dissertation, Technische Universität Dresden, Dresden, 2009.

Alriksson, B. Influence of site factors on Salix growth with emphasis on nitrogen response under different soil conditions. Dissertation, Swedish University of Agricultural Scciences, Uppsala, 1997. 
Amichev, B. Y., Johnston, M., and Van Rees, K. C. Hybrid poplar growth in bioenergy production systems: Biomass prediction with a simple process-based model (3pg). Biomass and Bioenergy, 34(5):687-702, May 2010. ISSN 09619534. doi: 10.1016/j.biombioe.2010.01.012. URL http://www.sciencedirect. com/science/article/pii/S0961953410000139.

Amichev, B. Y., Hangs, R. D., and Van Rees, K. C. A novel approach to simulate growth of multi-stem willow in bioenergy production systems with a simple process-based model (3pg). Biomass and Bioenergy, 35(1):473-488, January 2011. ISSN 0961-9534. doi: 10.1016/j.biombioe.2010.09.007. URL http://www.sciencedirect.com/science/article/pii/S0961953410003302.

Amthauer Gallardo, D. Standortbasierte Ertragsmodellierung von Pappel- und Weidenklonen in Kurzumtriebsplantagen. Dissertation, Technische Universität Dresden, Dresden, May 2014. URL http://www.qucosa.de/recherche/ frontdoor/?tx_slubopus4frontend [id] $=14467$.

Amthauer Gallardo, D. and Seymour, D. Rindenanteile und Aschegehalte von Pappel- und Weidenhybriden, September 2011. URL https: //www.nw-fva.de/fileadmin/user_upload/Verwaltung/Veranstaltungen/ 2011/10_AmthauerD_Rindenanteile_und_Aschegehalte_von_Pappel-_und_ Weidenhybriden.pdf.

Anonymous. Die Pappel - Klone, Klonmischungen und Familieneltern, May 2012. URL http://www.ble.de/SharedDocs/Downloads/02_Kontrolle/07_ SaatUndPflanzgut/Pappelklone_mischungen.pdf?__blob=publicationFile.

Anonymous. Erhebung zur Versorgungssituation von forstlichem Vermehrungsgut im Bundesgebiet (Erfassungszeitraum 01.07.2015 30.06.2016). Technical Report 324 - 2.2, Bundesanstalt für Landwirtschaft und Ernährung, Bonn, September 2016. URL http: //www.ble.de/SharedDocs/Downloads/02_Kontrolle/07_SaatUndPflanzgut/ Ernte2015_2016.pdf?_-_blob=publicationFile.

Anonymous. Erhebung zur Versorgungssituation von forstlichem Vermehrungsgut im Bundesgebiet (Erfassungszeitraum 01.07.2016 - 30.06.2017). Technical Report 324 - 2.2, Bundesanstalt für Landwirtschaft und Ernährung, 
Bonn, January 2018. URL https://www.ble.de/SharedDocs/Downloads/ DE/Landwirtschaft/Saat-und-Planzgut/Erhebung2016_2017.pdf?__blob= publicationFile\&v $=4$.

Arbeitskreis Standortskartierung in der Arbeitsgemeinschaft Forsteinrichtung. Forstliche Standortsaufnahme: Begriffe, Definitionen, Einteilungen, Kennzeichnungen, Erläuterungen. IHW-Verlag, Eching bei München, 7. auflage edition, 2016. ISBN 978-3-930167-80-7.

Aylott, M. J., Casella, E., Tubby, I., Street, N. R., Smith, P., and Taylor, G. Yield and spatial supply of bioenergy poplar and willow short-rotation coppice in the UK. New Phytologist, 178(2):358-370, April 2008. ISSN 1469-8137. doi: 10. 1111/j.1469-8137.2008.02396.x. URL http://onlinelibrary.wiley.com/doi/ $10.1111 / j .1469-8137.2008 .02396 . x /$ abstract.

Balasus, A. Umwelt- und Ertragswirkungen der Stickstoffdüngung beim Anbau von Weiden und Pappeln auf Ackerflächen unter Berücksichtigung phytopathologischer Aspekte. Dissertation, Technische Universität Dresden, Dresden, 2014. URL https://nbn-resolving.org/urn:nbn:de:bsz: 14-qucosa-158254.

Barbosa, A., Brown, J., Jimenez-Valverde, A., and Real, R. modEvA: Model Evaluation and Analysis, 2016. URL https://CRAN.R-project.org/package= modEvA. Version 1.3.2.

Bartels, H. Die Gattung Populus in dendrologischer Sicht. Die Holzzucht, 41(1/2): $1-7,1987$.

Bastien, R., Bohr, T., Moulia, B., and Douady, S. Unifying model of shoot gravitropism reveals proprioception as a central feature of posture control in plants. Proceedings of the National Academy of Sciences, 110(2):755-760, January 2013. ISSN 0027-8424, 1091-6490. doi: 10.1073/pnas.1214301109. URL http://www.pnas.org/cgi/doi/10.1073/pnas.1214301109.

Bayer, D., Seifert, S., and Pretzsch, H. Structural crown properties of Norway spruce (Picea abies [L.] Karst.) and European beech (Fagus sylvatica [L.]) in mixed versus pure stands revealed by terrestrial laser scanning. Trees, 27(4):1035-1047, August 2013. ISSN 0931-1890, 1432-2285. 
doi: 10.1007/s00468-013-0854-4. URL http://link.springer.com/10.1007/ s00468-013-0854-4.

Bergante, S., Facciotto, G., and Minotta, G. Identification of the main site factors and management intensity affecting the establishment of Short-RotationCoppices (SRC) in Northern Italy through stepwise regression analysis. Open Life Sciences, 5(4), January 2010. ISSN 2391-5412. doi: 10.2478/ s11535-010-0028-y. URL http://www.degruyter.com/view/j/biol.2010.5. issue-4/s11535-010-0028-y/s11535-010-0028-y.xml.

Biertümpfel, A., Rudel, H., Werner, A., and Vetter, A. 15 Jahre Energieholzversuche in Thüringen, 2009. URL http://www.tll.de/www/daten/ pflanzenproduktion/nawaro/feste_bio/ehol1009.pdf. Downloaded: 201906-02.

Biertümpfel, A., Graf, T., and Vetter, A. Feldversuchsbericht 2010 und 2011 - Ölfrüchte und Nachwachsende Rohstoffe. Trial Report, Thüringer Landesanstalt für Landwirtschaft, Dornburg, 2012.

Bilodeau-Gauthier, S., Paré, D., Messier, C., and Bélanger, N. Juvenile growth of hybrid poplars on acidic boreal soil determined by environmental effects of soil preparation, vegetation control, and fertilization. Forest Ecology and Management, 261(3):620-629, February 2011. ISSN 03781127. doi: 10.1016/ j.foreco.2010.11.016. URL http://linkinghub.elsevier.com/retrieve/pii/ S0378112710006869.

Bivand, R. S., Pebesma, E., and Gómez-Rubio, V. Applied Spatial Data Analysis with R. Springer New York, New York, NY, 2013. ISBN 978-1-4614-7617-7 9781-4614-7618-4. doi: 10.1007/978-1-4614-7618-4. URL http://link.springer. com/10.1007/978-1-4614-7618-4.

Blender Foundation. Blender, February 2012. URL https://download.blender. org/release/Blender2.62/. Version 2.62.

Blume, H.-P., Brümmer, G. W., Horn, R., Kandeler, E., Kögel-Knabner, I., Kretzschmar, R., Stahr, K., and Wilke, B.-M. Scheffer/Schachtschabel: Lehrbuch der Bodenkunde. Springer Berlin Heidelberg, Berlin, Heidelberg, 2010. ISBN 
978-3-662-49959-7 978-3-662-49960-3. doi: 10.1007/978-3-662-49960-3. URL http://link.springer.com/10.1007/978-3-662-49960-3.

Boelcke, B. and Kahle, P. Energieholzproduktion mit Weiden und Pappeln Ertragsbildung und Grundnährstoffbedarf. Pflanzenbauwissenschaften, 12(2): 78-85, 2008.

Boudon, F., Preuksakarn, C., Ferraro, P., Diener, J., Nacry, P., Nikinmaa, E., and Godin, C. Quantitative assessment of automatic reconstructions of branching systems obtained from laser scanning. Annals of Botany, 114(4): 853-862, September 2014. doi: 10.1093/aob/mcu062. URL http://aob. oxf ordjournals. org/content/114/4/853. abstract.

Bradshaw, H., Ceulemans, R., Davis, J., and Stettler, R. Emerging Model Systems in Plant Biology: Poplar (Populus) as A Model Forest Tree. Journal of Plant Growth Regulation, 19(3):306-313, September 2000. ISSN 0721-7595, 1435-8107. doi: 10.1007/s003440000030. URL http://link. springer.com/10. $1007 / \mathrm{s} 003440000030$.

Broeckx, L. S., Verlinden, M. S., Vangronsveld, J., and Ceulemans, R. Importance of crown architecture for leaf area index of different Populus genotypes in a high-density plantation. Tree Physiology, 32(10):1214-1226, October 2012. ISSN 0829-318X, 1758-4469. doi: 10.1093/treephys/tps083. URL http://treephys.oxfordjournals.org/cgi/doi/10.1093/treephys/tps083.

Buck-Sorlin, G. Functional-Structural Plant Modeling. In Dubitzky, W., Wolkenhauer, O., Cho, K.-H., and Yokota, H., editors, Encyclopedia of Systems Biology, pages 778-781. Springer New York, New York, NY, 2013a. ISBN 978-14419-9862-0 978-1-4419-9863-7. doi: 10.1007/978-1-4419-9863-7_1479. URL http://link.springer.com/10.1007/978-1-4419-9863-7_1479.

Buck-Sorlin, G. Lindenmayer System. In Dubitzky, W., Wolkenhauer, O., Cho, K.-H., and Yokota, H., editors, Encyclopedia of Systems Biology, pages 11271128. Springer New York, New York, NY, 2013b. ISBN 978-1-4419-9862-0 978-1-4419-9863-7. doi: 10.1007/978-1-4419-9863-7_1542. URL http://link. springer.com/10.1007/978-1-4419-9863-7_1542. 
Buck-Sorlin, G., Kniemeyer, O., and Kurth, W. Physiologie und Morphologie der Pappel (Populus sp.) modelliert mit Relationalen Wachstumsgrammatiken. In Wunn, U., editor, 17. Tagung der DVFFA Sektion Forstliche Biometrie und Informatik, Die Grüne Reihe, pages 1-11, Freiburg, 2005. Deutscher Verband Forstlicher Forschungsanstalten / Sektion Forstliche Biometrie und Informatik.

Buhler, D. D., Netzer, D. A., Riemenschneider, D. E., and Hartzler, R. G. Weed management in short rotation poplar and herbaceous perennial crops grown for biofuel production. Biomass and Bioenergy, 14(4):385-394, April 1998. ISSN 09619534. doi: 10.1016/S0961-9534(97)10075-7. URL https://linkinghub. elsevier.com/retrieve/pii/S0961953497100757.

Bungart, R. and Hüttl, R. Production of biomass for energy in post-mining landscapes and nutrient dynamics. Biomass and Bioenergy, 20(3):181-187, March 2001. ISSN 0961-9534. doi: 10.1016/S0961-9534(00)00078-7. URL http://www.sciencedirect.com/science/article/pii/S0961953400000787.

Bungart, R. and Hüttl, R. Growth dynamics and biomass accumulation of 8year-old hybrid poplar clones in a short-rotation plantation on a clayey-sandy mining substrate with respect to plant nutrition and water budget. European Journal of Forest Research, July 2004. ISSN 1612-4669, 1612-4677. doi: 10.1007/s10342-004-0024-8. URL http://link.springer.com/10.1007/ s10342-004-0024-8.

Bärwolff, M., Hansen, H., Hofmann, M., and Setzer, F. Energieholz aus der Landwirtschaft. Fachagentur Nachwachsende Rohstoffe e. V. (FNR), GülzowPrüzen, 5., vollst. überarb. aufl. edition, 2012. URL http://mediathek.fnr.de/ media/downloadable/files/samples/e/n/energieholz_dina5_web_4.pdf.

Böhm, C. and Veste, M. Agrarholzanbau im Kontext einer modernen Landwirtschaft. In Veste, M. and Böhm, C., editors, Agrarholz - Schnellwachsende Bäume in der Landwirtschaft, pages 57-77. Springer Berlin Heidelberg, Berlin, Heidelberg, 2018. ISBN 978-3-662-49930-6 978-3-662-49931-3. doi: 10.1007/978-3-662-49931-3_5. URL http://link.springer.com/10.1007/ 978-3-662-49931-3_5.

Böhm, C., Quinkenstein, A., Freese, D., and Hüttl, R. F. Assessing the short 
rotation woody biomass production on marginal post-mining areas. Journal of Forest Science, 57(7):303-311, 2011.

Caraglio, Y. and Barthélémy, D. Revue critique des termes relatifs à la croissance et à la ramification des tiges des végétaux vasculaires. In Bouchon, J., De Reffye, P., and Barthélémy, D., editors, Modélisation et simulation de l'architecture des végétaux, Science update, pages 11-87. INRA, Montpellier, France, 1997. ISBN 978-2-7380-0706-3.

Casella, E. and Sinoquet, H. A method for describing the canopy architecture of coppice poplar with allometric relationships. Tree Physiology, 23:1153-1170, 2003. URL http://treephys.oxfordjournals .org/.

Caslin, B., Finnan, J., and McCracken, A. Willow varietal identification guide. Teagasc; AFBI, Carlow; Belfast, September 2012. ISBN 10 1-84170590-X. URL https://www.teagasc.ie/media/website/publications/2012/ Willow_Identification_Guide_2012.pdf.

Ceulemans, R., McDonald, A., and Pereira, J. A comparison among eucalypt, poplar and willow characteristics with particular reference to a coppice, growthmodelling approach. Modelling Short Rotation Forestry Growth, 11(2-3):215231, 1996. ISSN 0961-9534. doi: 10.1016/0961-9534(96)00035-9. URL http: //www.sciencedirect.com/science/article/pii/0961953496000359.

Ceulemans, R., Stettler, R. F., Hinckley, T. M., Isebrands, J. G., and Heilman, P. E. Crown architecture of Populus clones as determined by branch orientation and branch characteristics. Tree Physiology, 7(1-2-3-4):157-167, December 1990. ISSN 0829-318X, 1758-4469. doi: 10.1093/treephys/7.1-2-3-4. 157. URL https://academic.oup.com/treephys/article-lookup/doi/10. 1093/treephys/7.1-2-3-4.157.

Ceulemans, R. J. Genetic variation in functional and structural productivity determinants in poplar. Thesis Publ. [u.a.], Amsterdam, 1990. ISBN 978-90-5170044-2.

Chambelland, J.-C., Dassot, M., Adam, B., Donès, N., Balandier, P., Marquier, A., Saudreau, M., Sonohat, G., and Sinoquet, H. A double-digitising method 
for building 3d virtual trees with non-planar leaves: application to the morphology and light-capture properties of young beech trees ( Fagus sylvatica ). Functional Plant Biology, 35(10):1059, 2008. ISSN 1445-4408. doi: 10.1071/FP08051. URL http://www.publish.csiro.au/?paper=FP08051.

Chuine, I., Cambon, G., and Comtois, P. Scaling phenology from the local to the regional level: advances from species-specific phenological models. Global Change Biology, 6(8):943-952, December 2000. ISSN 1354-1013, 1365-2486. doi: 10.1046/j.1365-2486.2000.00368.x. URL http://doi.wiley.com/10.1046/ j.1365-2486.2000.00368.x.

Chuine, I. and Beaubien, E. G. Phenology is a major determinant of tree species range. Ecology Letters, 4(5):500-510, September 2001. ISSN 1461-023X, 1461-0248. doi: 10.1046/j.1461-0248.2001.00261.x. URL http://doi.wiley. com/10.1046/j.1461-0248.2001.00261.x.

Cline, M. G. and Dong-IL, K. A preliminary investigation of the role of auxin and cytokinin in sylleptic branching of three hybrid poplar clones exhibiting contrasting degrees of sylleptic branching. Annals of Botany, 90(3):417-421, September 2002. ISSN 0305-7364.

Corona, P., Antonio Marziliano, P., and Scotti, R. Top-down growth modelling: a prototype for poplar plantations in Italy. Forest Ecology and Management, 161 (1-3):65-73, May 2002. ISSN 03781127. doi: 10.1016/S0378-1127(01)00481-9. URL http://linkinghub.elsevier.com/retrieve/pii/S0378112701004819.

Coxeter, H. S. M. Introduction to geometry. Wiley, New York, 2d ed edition, 1969. ISBN 978-0-471-18283-2.

Critchfield, W. B. LEAF DIMORPHISM IN POPULUS TRICHOCARPA. American Journal of Botany, 47(8):699-711, October 1960. ISSN 00029122. doi: 10.1002/j.1537-2197.1960.tb07154.x. URL http://doi.wiley.com/10.1002/j. 1537-2197.1960.tb07154.x.

Cronk, Q. C. B. Plant eco-devo: the potential of poplar as a model organism: Research review. New Phytologist, 166(1):39-48, February 2005. ISSN 0028646X, 14698137. doi: 10.1111/j.1469-8137.2005.01369.x. URL http: //doi.wiley.com/10.1111/j.1469-8137.2005.01369.x. 
Crow, P. and Houston, T. The influence of soil and coppice cycle on the rooting habit of short rotation poplar and willow coppice. Biomass and Bioenergy, 26 (6):497-505, June 2004. ISSN 09619534. doi: 10.1016/j.biombioe.2003.09.002. URL http://linkinghub.elsevier.com/retrieve/pii/S096195340300165X.

Dahl, D. B., Scott, D., Roosen, C., Magnusson, A., and Swinton, J. xtable: Export Tables to LaTeX or HTML, 2019. URL https://CRAN. R-project . org/package= xtable. Version 1.8-4.

Danjon, F. and Reubens, B. Assessing and analyzing 3d architecture of woody root systems, a review of methods and applications in tree and soil stability, resource acquisition and allocation. Plant and Soil, 303(1-2):1-34, February 2008. ISSN 0032-079X, 1573-5036. doi: 10.1007/s11104-007-9470-7. URL http://link.springer.com/10.1007/s11104-007-9470-7.

Deckmyn, G., Laureysens, I., Garcia, J., Muys, B., and Ceulemans, R. Poplar growth and yield in short rotation coppice: model simulations using the process model SECRETS. Biomass and Bioenergy, 26(3):221-227, March 2004. ISSN 09619534. doi: 10.1016/S0961-9534(03)00121-1. URL http://linkinghub. elsevier.com/retrieve/pii/S0961953403001211.

Dickmann, D. I. and Kuzovkina, J. Poplars and Willows of the World, With Emphasis on Silviculturally Important Species. In Isebrands, J. and Richardson, J., editors, Poplars and willows: trees for society and the environment, pages 8-91. CABI ; FAO, Boston, MA : Rome, 2014. ISBN 978-1-78064-108-9 97892-5-107185-4. URL http://www .fao.org/3/a-i2670e.pdf.

Dickmann, D. I., Isebrands, J., Blake, T. J., Kosola, K., and Kort, J. Physiological ecology of poplars. In Poplar culture in North America, pages 77-115. NRC Research Press, Ottawa, Ont., 2002. ISBN 978-0-660-18145-5 978-0-660-189888. URL http://nrcresearchpress . com/doi/book/10.1139/9780660181455.

Dimitriou, I. and Mola-Yudego, B. Nitrogen fertilization of poplar plantations on agricultural land: effects on diameter increments and leaching. Scandinavian Journal of Forest Research, 32(8):700-707, November 2017. ISSN 0282-7581, 1651-1891. doi: 10.1080/02827581.2016.1264622. URL https: //www.tandfonline.com/doi/full/10.1080/02827581.2016.1264622. 
DIN Deutsches Institut für Normung e.V. Feste Biobrennstoffe - Bestimmung des Wassergehaltes - Ofentrocknung - Teil 1: Gesamtgehalt an Wasser - Referenzverfahren; Deutsche Fassung EN 14774-1:2009, February 2010. URL https://www.beuth.de/de/norm/din-en-14774-1/122640083.

Dinis, C. CORK OAK (Quercus suber L.) ROOT SYSTEM: A STRUCTURALFUNCTIONAL 3D APPROACH. Dissertation, Universidade de Évora, Évora, November 2014. URL http://hdl.handle.net/10174/17314.

Donès, N., Adam, B., and Sinoquet, H. PiafDigit-software to drive a Polhemus Fastrak 3 SPACE 3d digitiser and for the acquisition of plant architecture, 2006. Version 1.0.

Dryden, I. L. and Mardia, K. V. Statistical shape analysis with applications in $R$. John Wiley \& Sons Ltd, Chichester [West Sussex], 2016. ISBN 978-1-11907249-2 978-1-119-07251-5 978-1-119-07250-8. URL http://public.eblib. com/choice/publicfullrecord . aspx?p=4573538

Dryden, I. L. shapes: Statistical Shape Analysis, 2018. URL https://CRAN. R-project.org/package=shapes, Version 1.2.4.

Duhme, H. Die Standortansprüche der Pappeln: ein Leitfaden für Anbauer und Berater, 1989.

Eisenhauer, J. G. Regression through the Origin. Teaching Statistics, 25(3):7680, September 2003. ISSN 0141-982X, 1467-9639. doi: 10.1111/1467-9639. 00136. URL http://doi.wiley.com/10.1111/1467-9639.00136.

Elewa, A. M., editor. Morphometrics for Nonmorphometricians, volume 124 of Lecture Notes in Earth Sciences. Springer Berlin Heidelberg, Berlin, Heidelberg, 2010. ISBN 978-3-540-95852-9 978-3-540-95853-6. doi: 10.1007/978-3-540-95853-6. URL http://link.springer.com/10.1007/ 978-3-540-95853-6.

Eysn, L., Pfeifer, N., Ressl, C., Hollaus, M., Grafl, A., and Morsdorf, F. A Practical Approach for Extracting Tree Models in Forest Environments Based on Equirectangular Projections of Terrestrial Laser Scans. Remote Sensing, 5 
(11):5424-5448, October 2013. ISSN 2072-4292. doi: 10.3390/rs5115424. URL http://www.mdpi.com/2072-4292/5/11/5424.

Fabrika, M. Simulátor biodynamiky lesa SIBYLA. Koncepcia, konštrukcia a programové riešenie. Habilitation thesis, Technická univerzita vo Zvolene, Zvolen, 2005.

Fabrika, M. and Pretzsch, H. FOREST ECOSYSTEM ANALYSIS AND MODELLING. Technical University Zvolen, Zvolen, 2013. ISBN 978-80-228-2506-1.

Fahrmeir, L., Kneib, T., Lang, S., and Marx, B. Regression. Springer Berlin Heidelberg, Berlin, Heidelberg, 2013. ISBN 978-3-642-34332-2 978-3-64234333-9. URL http://link. springer.com/10.1007/978-3-642-34333-9.

Faraway, J. J. Linear models with $R$. Texts in statistical science. Chapman and Hall/CRC, Boca Raton, FL, 2015. ISBN 978-1-4398-8734-9. URL http://www. crcnetbase.com/isbn/9781439887349.

Faraway, J. J. Extending the linear model with R: generalized linear, mixed effects and nonparametric regression models. Texts in statistical science. Chapman \& Hall/CRC, Boca Raton, FL, 2016. ISBN 978-1-4987-2099-1. URL https:// nls.ldls.org.uk/welcome.html?ark:/81055/vdc_100044258535.0x000001.

Fell, D. Die Propagierung des Pappelanbaues in den 50er Jahren - Versuch eine Wertung anhand der Literatur. Diploma thesis, Georg-August Universität Göttingen, Göttingen, August 1992.

Ferm, A., Hytönen, J., and Vuori, J. Effect of spacing and nitrogen fertilization on the establishment and biomass production of short rotation poplar in Finland. Biomass, 18(2):95-108, January 1989. ISSN 01444565. doi: 10. 1016/0144-4565(89)90087-5. URL http://www.sciencedirect.com/science/ article/pii/0144456589900875.

Fey, C., Stiehm, C., Hofmann, M., Moos, M., Grotehusmann, H., Steiner, W., and Janßen, A. Züchtung und Evaluierung von Schwarz- und Balsampappeln hinsichtlich Ertrag und Blattrosttoleranz in vier FastWOOD Versuchsserien. Landbauforschung - applied agricultural and forestry research, (online 
first):online first, September 2018. doi: 10.3220/LBF1534945636000. URL https://literatur.thuenen.de/digbib_extern/dn060146.pdf.

Fischer, C. Comparing the Logarithmic Transformation and the Box-Cox Transformation for Individual Tree Basal Area Increment Models. Forest Science, 62 (3):297-306, June 2016. ISSN 0015-749X. doi: 10.5849/forsci.15-135. URL http://www.ingentaconnect.com/content/10.5849/forsci.15-135.

Fitchett, J. M., Grab, S. W., and Thompson, D. I. Plant phenology and climate change: Progress in methodological approaches and application. Progress in Physical Geography, 39(4):460-482, August 2015. ISSN 0309-1333, 14770296. doi: 10.1177/0309133315578940. URL http://journals.sagepub.com/ doi/10.1177/0309133315578940.

FORMATH Research Group. FastrakDigitizer, 2013. URL http://www.formath . jp/software/FastrakDigitizerSetup.msi. Version 1.0.

Fox, J. and Weisberg, S. An $R$ companion to applied regression. Sage Publications, Inc, Thousand Oaks, California, third edition edition, 2019. ISBN 978-1-5443-3647-3.

Gadow, K. v. Waldstruktur und Wachstum: [Beilage zur Vorlesung im Wintersemester 2003/2004]. Universitätsdrucke Göttingen. Univ.-Verl. Göttingen, Göttingen, 2003. ISBN 3-930457-32-6 3-930457-32-6 978-3-930457-32-8.

Gang, Y., Weiqun, C., Dongxiang, L., Kang, M. Z., Letort, V., Meijun, X., and Xinyuan, $\mathrm{H}$. Growth and development simulation based on functional-structural model GreenLab for poplar (Salicaceae). African Journal of Agricultural Research, 6(13):3071-3077, 2011.

Gebauer, R., Vanbeveren, S. P., Volařík, D., Plichta, R., and Ceulemans, R. Petiole and leaf traits of poplar in relation to parentage and biomass yield. Forest Ecology and Management, 362:1-9, February 2016. ISSN 03781127. doi: 10.1016/j.foreco.2015.11.036. URL http://linkinghub.elsevier.com/ retrieve/pii/S0378112715006593.

Ghelardini, L., Berlin, S., Weih, M., Lagercrantz, U., Gyllenstrand, N., and Rönnberg-Wästljung, A. C. Genetic architecture of spring and autumn phenology in Salix. BMC Plant Biology, 14(1):31, 2014. ISSN 1471-2229. doi: 
10.1186/1471-2229-14-31. URL http://www.biomedcentral.com/1471-2229/ $14 / 31$.

Godin, C., Costes, E., and Sinoquet, H. A Method for Describing Plant Architecture which Integrates Topology and Geometry. Annals of Botany, 84 (3):343-357, September 1999. ISSN 03057364. doi: 10.1006/anbo.1999. 0923. URL https://academic.oup.com/aob/article-lookup/doi/10.1006/ anbo.1999.0923.

Godin, C., Guédon, Y., Costes, E., and Caraglio, Y. Measuring and analysing plants with the AMAPmod software. In Plants to Ecosystems, volume 1 of Advances in Computational Life Sciences, pages 53-84. CSIRO, Collingwood, Vic., 1997.

Google Satellite. Google Satellite image of Witzenhausen and surrounding area., 2015. URL https://www.google.com. Downloaded: 2018-12-28.

GrolMP Developer Team. GrolMP, November 2016. URL https://sourceforge. net/projects/groimp/files/groimp/1.5/. Version 1.5.

Grotehusmann, H., Janßen, A., Haikali, A., Hartmann, K.-U., Hüller, W., Karopka, M., Schildbach, M., Schirmer, R., Schuppelius, T., and Töpfner, K. Pappelsortenprüfungen im Projekt FastWOOD. Forstarchiv, (86):67-79, August 2015. ISSN 0300-4112. doi: 10.4432/0300-4112-86-67. URL http://www.forstpraxis.de/ schnellwachsende-baumarten-zuechtung-pappeln-sortenpruefung-kurzumtriebsplantas

Grotehusmann, H., Stiehm, C., Janßen, A., Hartmann, K.-U., List, J., Karopka, M., Moos, M., Schildbach, M., and Schirmer, R. Pappelsortenprüfungen im Projekt FastWOOD - Ergebnisse nach 2. Rotation. Forstarchiv, pages 47-54, May 2017. ISSN 0300-4112. doi: 10.4432/0300-4112-88-47. URL https://www.forstpraxis.de/ schnellwachsende-baumarten-zuechtung-pappeln-sortenpruefung-kurzumtriebsplantas

Guillon, T., Dumont, Y., and Fourcaud, T. A new mathematical framework for modelling the biomechanics of growing trees with rod theory. Mathematical and Computer Modelling, 55(9-10):2061-2077, May 2012. ISSN 08957177. doi: 10. 
1016/j.mcm.2011.12.024. URL http://linkinghub.elsevier.com/retrieve/ pii/S0895717711007874.

Guisan, A. and Zimmermann, N. E. Predictive habitat distribution models in ecology. Ecological Modelling, 135(2-3):147-186, December 2000. ISSN 03043800. doi: 10.1016/S0304-3800(00)00354-9. URL http://linkinghub. elsevier.com/retrieve/pii/S0304380000003549.

Hallé, F., Oldeman, R. A. A., and Tomlinson, P. B. Tropical Trees and Forests. Springer Berlin Heidelberg, Berlin, Heidelberg, 1978. ISBN 978-3-642-811920 978-3-642-81190-6. doi: 10.1007/978-3-642-81190-6. URL http://link. springer.com/10.1007/978-3-642-81190-6.

Hammes, W. Wachstum, Wasserverbrauch und Produktivität des Wasserverbrauchs von Pappeln unterschiedlichen Alters : Populus $x$ euramericana (Dode) Guinier c.v. Robusta. Dissertation, Albert-Ludwigs-Universität Freiburg, Freiburg im Breisgau, 1983.

Hansen, E. A. Planting Date Affects Survival and Height Growth of Hybrid Poplar. The Forestry Chronicle, 62(3):164-169, June 1986. ISSN 0015-7546, 14999315. doi: 10.5558/tfc62164-3. URL http://pubs.cif-ifc.org/doi/abs/10. 5558/tfc62164-3.

Hansen, J. and Nagel, J. Waldwachstumskundliche Softwaresysteme auf Basis von TreeGrOSS - Anwendung und theoretische Grundlagen. Univ.-Verl. Göttingen, Göttingen, 2014. ISBN 978-3-86395-149-8 3-86395-149-2.

Hartmann, K.-U. Entwicklung eines Ertragsschätzers für Kurzumtriebsbestände aus Pappel. Dissertation, Technische Universität Dresden, Dresden, August 2010. URL http://nbn-resolving.de/urn:nbn:de:bsz:14-qucosa-62853.

Headlee, W. L., Zalesny, R. S., Donner, D. M., and Hall, R. B. Using a ProcessBased Model (3-PG) to Predict and Map Hybrid Poplar Biomass Productivity in Minnesota and Wisconsin, USA. BioEnergy Research, 6(1):196-210, March 2013. ISSN 1939-1234, 1939-1242. doi: 10.1007/s12155-012-9251-x. URL http://link.springer.com/10.1007/s12155-012-9251-x. 
Henke, M. and Sloboda, B. Semiautomatic tree ring segmentation using Active Contours and an optimised gradient operator / Poloautomatizovaná segmentácia ročných radiálnych kruhov stromov s využitím metódy „Active Contours“ a optimalizovaného gradientového operátora. Forestry Journal, 60(3):185-190, September 2014. ISSN 0323-1046. doi: 10.2478/forj-2014-0020. URL http: //content.sciendo.com/view/journals/forj/60/3/article-p185.xml.

Henke, M., Huckemann, S., Kurth, W., and Sloboda, B. Reconstructing leaf growth based on non-destructive digitizing and low-parametric shape evolution for plant modelling over a growth cycle. Silva Fennica, 48(2), 2014. ISSN 22424075. doi: 10.14214/sf.1019. URL http://www.silvafennica.fi/ article/1019.

Heyn, N. and Wachendorf, C. Erfassung von Klon-Standort-Wechselwirkungen bei Pappel und Weide auf landwirtschaftlichen Standorten in kurzen Umtriebszeiten - ProLoc - Teilvorhaben: Kohlenstoffdynamik in Böden von Kurzumtriebsplantagen. Final Report, Fachgebiet Bodenbiologie und Pflanzenernährung am Fachbereich Ökologische Agrarwissenschaften der Universität Kassel, 2012.

Hickey, L. J. CLASSIFICATION OF THE ARCHITECTURE OF DICOTYLEDONOUS LEAVES. American Journal of Botany, 60(1):17-33, January 1973. ISSN 00029122. doi: 10.1002/j.1537-2197.1973.tb10192.x. URL http://doi. wiley.com/10.1002/j.1537-2197.1973.tb10192.x.

Hijmans, R., Kapoor, J., Wieczorek, J., Garcia, N., Maunahan, A., Rala, A., and Mandel, A. GADM database of Global Administrative Areas, November 2015. URL http://gadm.org/. Last visited: 2016-03-21.

Hoffmann, G. Verlauf der Tiefendurchwurzelung und Feinwurzelbildung bei einigen Baumarten. Archiv für Forstwesen, 15(8):825-856, 1966.

Hofmann, M. Pappeln als nachwachsender Rohstoff auf Ackerstandorten Kulturverfahren, Ökologie und Wachstum unter dem Aspekt der Sortenwahl. Number 8 in Schriften des Forschungsinstitutes für schnellwachsende Baumarten Hann. Münden. Forschungsinstitut für schnellwachsende Baumarten, Hannoversch Münden, 2005. ISBN 978-3-927194-05-2. 
Hofmann-Schielle, C., Jug, A., Makeschin, F., and Rehfuess, K. Short-rotation plantations of balsam poplars, aspen and willows on former arable land in the Federal Republic of Germany. I. Site-growth relationships. Forest Ecology and Management, 121(1-2):41-55, 1999. doi: 10.1016/S0378-1127(98)00555-6.

Horn, H., Skibbe, K., and Röhle, H. Wuchsleistung von KUP aus Pappel in Folgerotationen. AFZ-DerWald, (7):53-55, 2013. URL http://www.researchgate.net/profile/Hendrik_Horn/publication/ 275155989_Wuchsleistung_von_KUP_aus_Pappel_in_Folgerotationen/ links/55348bc90cf2f2a588b25de7.pdf.

IntelliJ Developer Team. IntelliJ IDEA (Ultimate Edition), 2019. URL https:// www. jetbrains.com/. Version 2019.1.3.

Janßen, A., Fey-Wagner, C., and Hofmann, M. Verbundvorhaben FASTWOOD: Züchtung schnellwachsender Baumarten-Teil Pappeln. Berlin, May 2010. URL http://www.nwfva.de/fileadmin/user_upload/Verwaltung/ Publikationen/2010/09_02_Beitrag_Janssen_Agrarholz_2010.pdf.

Janßen, A., Stiehm, C., Hofmann, M., and Schmidt, C. Erfassung von KlonStandort-Wechselwirkungen bei Pappel und Weide auf landwirtschaftlichen Standorten in kurzen Umtriebszeiten - ProLoc (Ertrags - Programm - Locus) - Projektphase II : Schlussbericht zum Vorhaben : Laufzeit: 04.05.2012 bis 31.12.2015. Final Report, Nordwestdeutsche Forstliche Versuchsanstalt Abteilung Waldgenressourcen, 2017.

Jirasek, C., Prusinkiewicz, P., and Moulia, B. Integrating biomechanics into developmental plant models expressed using L-systems. In Proceedings of the 3rd Plant Biomechanics Conference, pages 615-624, Freiburg-Badenweiler, 2000. Georg Thieme, Stuttgart.

Kadereit, J. W., Körner, C., Kost, B., and Sonnewald, U. Strasburger - Lehrbuch der Pflanzenwissenschaften. Springer Berlin Heidelberg, Berlin, Heidelberg, 2014. ISBN 978-3-642-54434-7 978-3-642-54435-4. doi: 10.1007/978-3-642-54435-4. URL http://link.springer.com/10.1007/ 978-3-642-54435-4 
Kahlen, K. and Stützel, H. Estimation of Geometric Attributes and Masses of Individual Cucumber Organs Using Three-dimensional Digitizing and Allometric Relationships. Journal of the American Society for Horticultural Science, 132(4):439-446, July 2007. ISSN 0003-1062, 2327-9788. doi: 10.21273/

JASHS.132.4.439. URL https://journals.ashs.org/view/journals/jashs/ 132/4/article-p439.xm1.

Keller, S. R., Soolanayakanahally, R. Y., Guy, R. D., Silim, S. N., Olson, M. S., and Tiffin, P. Climate-driven local adaptation of ecophysiology and phenology in balsam poplar, Populus balsamifera L. (Salicaceae). American Journal of Botany, 98(1):99-108, January 2011. ISSN 0002-9122, 1537-2197. doi: 10. 3732/ajb.1000317. URL http://doi.wiley.com/10.3732/ajb.1000317.

Kimball, S., Mattis, P., and GIMP Development Team. GNU Image Manipulation Program (GIMP), 2017. URL https://www.gimp.org/, Version 2.8.22.

Kniemeyer, O. Design and implementation of a graph grammar based language for functional-structural plant modelling. Dissertation, Brandenburgische Technische Universität, Cottbus, 2008. URL http://opus.kobv.de/btu/ volltexte/2009/593/.

Knust, C. Kurzumtriebsplantagen - Stand des Wissens. In Reeg, T., Bemmann, A., Konold, W., Murach, D., and Spiecker, H., editors, Anbau und Nutzung von Bäumen auf landwirtschaftlichen Flächen, pages 3-7. Wiley-VCH, Weinheim, 2009. ISBN 978-3-527-32417-0.

Koski, V. and Dickmann, D. Tree ideotype. International Energy Agency Bioenergy Agreement Progress and Achievements 1989-1991, 2(1-6):71-75, 1992. ISSN 0961-9534. doi: 10.1016/0961-9534(92)90089-9. URL http: //www.sciencedirect.com/science/article/pii/0961953492900899.

Krabel, D., Groschke, M., and Korn, S. Topfversuche mit drei Pappelstandardsorten - Möglichkeiten und Herausforderungen. Landbauforschung - applied agricultural and forestry research, 68(1/2):11-18, December 2018. doi: 10. 3220/LBF1530776106000. URL http://doi .org/10.3220/LBF1530776106000.

Kramer, H. and Akça, A. Leitfaden zur Waldmesslehre. Sauerländer, J D, Frankfurt am Main, 5 edition, 2008. ISBN 978-3-7939-0880-7. 
Kurth, W. and Anzola Jürgenson, G. Triebwachstum und Verzweigung junger Fichten in Abhängigkeit von den beiden Einflußgrößen Beschattung und Wuchsdichte: Datenaufbereitung und analyse mit GROGRA. In Deutscher Verband Forstlicher Forschungsanstalten, Sektion Forstliche Biometrie und Informatik, volume 10 of IUFRO - Die grüne Reihe, pages 89-108, Freiburg, 1997.

Kurth, W. Growth grammar interpreter grogra 2.4-a software tool for the 3dimensional interpretation of stochastic, sensitive growth grammars in the context of plant modelling. Technical Report 38, Forschungszentrum Waldökosysteme der Universität Göttingen, Göttingen, 1994a.

Kurth, W. Morphological models of plant growth: Possibilities and ecological relevance. Ecological Modelling, 75-76:299-308, September 1994b. ISSN 03043800. doi: 10.1016/0304-3800(94)90027-2. URL https://linkinghub. elsevier.com/retrieve/pii/0304380094900272.

Kurth, W. Die Simulation der Baumarchitektur mit Wachstumsgrammatiken: stochastische, sensitive L-Systeme als formale Basis für dynamische, morphologische Modelle der Verzweigungsstruktur von Gehölzen. Wissenschaftl. Verl, Berlin, 1999. ISBN 978-3-932089-37-4.

Kurth, W. Das dtd-Datenformat, April 2010. URL http://www.uni-forst.gwdg. de/ ${ }^{\sim}$ wkurth/dtd_code.pdf. Downloaded: 2019-04-30.

Kurth, W. Hinweise zu Messungen; L-Systeme, einfache Verzweigungsmodelle, May 2014. URL http://www.uni-forst.gwdg.de/〜wkurth/sm14_v03. ppt. Last visited: 2019-01-13.

Kurth, W. and Sloboda, B. Growth grammars simulating trees - an extension of L-systems incorporating local variables and sensitivity. Silva Fennica, 31(3), 1997. ISSN 22424075. doi: 10.14214/sf.a8527. URL http: //www.silvafennica.fi/article/5626.

Kurth, W., Kniemeyer, O., and Buck-Sorlin, G. Relational Growth Grammars - A Graph Rewriting Approach to Dynamical Systems with a Dynamical Structure. In Hutchison, D., Kanade, T., Kittler, J., Kleinberg, J. M., Mattern, F., Mitchell, 
J. C., Naor, M., Nierstrasz, O., Pandu Rangan, C., Steffen, B., Sudan, M., Terzopoulos, D., Tygar, D., Vardi, M. Y., Weikum, G., Banâtre, J.-P., Fradet, P., Giavitto, J.-L., and Michel, O., editors, Unconventional Programming Paradigms, volume 3566, pages 56-72. Springer Berlin Heidelberg, Berlin, Heidelberg, 2005. ISBN 978-3-540-27884-9 978-3-540-31482-0. doi: 10.1007/11527800_5. URL http://link.springer.com/10.1007/11527800_5.

Kutschera, L. and Lichtenegger, E. Wurzelatlas mitteleuropäischer Waldbäume und Sträucher. Number 6 in Wurzelatlas-Reihe. Stocker, Graz, 2. aufl edition, 2013. ISBN 978-3-7020-0928-1.

Kędra, K., Barbeito, I., Dassot, M., Vallet, P., and Gazda, A. Single-image photogrammetry for deriving tree architectural traits in mature forest stands: a comparison with terrestrial laser scanning. Annals of Forest Science, 76(1): 5, March 2019. ISSN 1286-4560, 1297-966X. doi: 10.1007/s13595-018-0783-x. URL http://link.springer.com/10.1007/s13595-018-0783-x.

Labrecque, M. and Teodorescu, T. I. Influence of plantation site and wastewater sludge fertilization on the performance and foliar nutrient status of two willow species grown under SRIC in southern Quebec (Canada). Forest Ecology and Management, 150(3):223-239, September 2001. ISSN 03781127. doi: 10.1016/S0378-1127(00)00567-3. URL http://linkinghub.elsevier.com/ retrieve/pii/S0378112700005673.

Lakämper, N. Protokoll zu den morphologischen Detailaufnahmen auf einer Versuchsfläche des ProLoc Verbundvorhaben an der Nordwestdeutschen Forstlichen Versuchsanstalt - Abteilung Waldgenressourcen. Project assignment, Georg-August Universität Göttingen, Göttingen, February 2014.

Landgraf, D., Bärwolff, M., Burger, F., Pecenka, R., Hering, T., and Schweier, J. Produktivität, Management und Nutzung von Agrarholz. In Veste, M. and Böhm, C., editors, Agrarholz - Schnellwachsende Bäume in der Landwirtschaft, pages 447-510. Springer Berlin Heidelberg, Berlin, Heidelberg, 2018. ISBN 9783-662-49930-6 978-3-662-49931-3. doi: 10.1007/978-3-662-49931-3_14. URL http://link.springer.com/10.1007/978-3-662-49931-3_14.

Landsberg, J. and Waring, R. A generalised model of forest productivity using 
simplified concepts of radiation-use efficiency, carbon balance and partitioning. Forest Ecology and Management, 95(3):209-228, August 1997. ISSN 03781127. doi: 10.1016/S0378-1127(97)00026-1. URL https://linkinghub. elsevier.com/retrieve/pii/S0378112797000261.

L'Ecuyer, P. SSJ: Stochastic Simulation in Java, October 2018. URL http:// simul.iro.umontreal.ca/ssj/indexe.html. Version 3.3.1.

Liang, X., Kankare, V., Hyyppä, J., Wang, Y., Kukko, A., Haggrén, H., Yu, X., Kaartinen, H., Jaakkola, A., Guan, F., Holopainen, M., and Vastaranta, M. Terrestrial laser scanning in forest inventories. ISPRS Journal of Photogrammetry and Remote Sensing, 115:63-77, May 2016. ISSN 09242716. doi: 10.1016/j.isprsjprs.2016.01.006. URL https://linkinghub.elsevier.com/ retrieve/pii/S0924271616000204.

Liesebach, M. and Schneck, V. Züchtung, Zulassungen, Vermehrung. In Veste, M. and Böhm, C., editors, Agrarholz - Schnellwachsende Bäume in der Landwirtschaft, pages 119-145. Springer Berlin Heidelberg, Berlin, Heidelberg, 2018. ISBN 978-3-662-49930-6 978-3-662-49931-3. doi: 10.1007/978-3-662-49931-3_5. URL http://link.springer.com/10.1007/ 978-3-662-49931-3_5.

Lindenmayer, A. Mathematical models for cellular interactions in development I. Filaments with one-sided inputs. Journal of Theoretical Biology, 18(3):280299, March 1968. ISSN 00225193. doi: 10.1016/0022-5193(68)90079-9. URL https://linkinghub.elsevier.com/retrieve/pii/0022519368900799.

Lindroth, A., Verwijst, T., and Halldin, S. Water-use efficiency of willow: Variation with season, humidity and biomass allocation. Journal of Hydrology, 156(1-4): 1-19, April 1994. ISSN 00221694. doi: 10.1016/0022-1694(94)90068-X. URL http://linkinghub.elsevier.com/retrieve/pii/002216949490068X.

Marron, N., Villar, M., Dreyer, E., Delay, D., Boudouresque, E., Petit, J.-M., Delmotte, F. M., Guehl, J.-M., and Brignolas, F. Diversity of leaf traits related to productivity in 31 Populus deltoides $\times$ Populus nigra clones. Tree Physiology, 25(4):425-435, April 2005. ISSN 0829-318X. doi: 10.1093/treephys/25.4.425. URL http://treephys . oxf ord journals .org/content/25/4/425. short. 
Marron, N., Bastien, C., Sabatti, M., Taylor, G., and Ceulemans, R. Plasticity of growth and sylleptic branchiness in two poplar families grown at three sites across Europe. Tree Physiology, 26(7):935-946, July 2006. ISSN 0829-318X.

Marron, N., Dillen, S. Y., and Ceulemans, R. Evaluation of leaf traits for indirect selection of high yielding poplar hybrids. Environmental and Experimental Botany, 61(2):103-116, November 2007. ISSN 00988472. doi: 10.1016/ j.envexpbot.2007.04.002. URL http://linkinghub.elsevier.com/retrieve/ pii/S0098847207000767.

Menzel, A. Phänologie von Waldbäumen unter sich ändernden Klimabedingungen: Auswertung der Beobachtungen in den internationalen phänologischen Gärten und Möglichkeiten der Modellierung von Phänodaten. Number 164 in Forstliche Forschungsberichte München. Heinrich Frank, München, 1997.

Moritz, S. and Bartz-Beielstein, T. imputeTS: Time Series Missing Value Imputation in R. The $R$ Journal, 9(1):207, 2017. ISSN 2073-4859. doi: 10.32614/RJ-2017-009. URL https://journal.r-project.org/archive/2017/ RJ-2017-009/index.html.

Murach, D., Murn, Y., and Hartmann, H. Ertragsermittlung und Potenziale von Agrarholz - Yield modelling and potentials of short rotation coppices (SRC). Forst und Holz, 63(6), 2008.

Murach, D., Hartmann, H., Murn, Y., Schultze, M., Wael, A., and Röhle, H. Standortsbasierte Leistungsschätzung in Agrarholzbeständen in Brandenburg und Sachsen. In Anbau und Nutzung von Bäumen auf landwirtschaftlichen Flächen, pages 29-40. Wiley-VCH, Weinheim, 2009. ISBN 978-3-527-32417-0.

Nagel, J. and Biging, G. S. Schätzung der Parameter der Weibullfunktion zur Generierung von Durchmesserverteilungen. Allgemeine Forst-und Jagdzeitung, 166(9-10):185-189, 1995.

Nagel, J., Albert, M., and Schmidt, M. Das waldbauliche Prognose- und Entscheidungsmodell BWINPro 6.1. Forst und Holz, 57(15/16):486-493, 2002.

Nagelkerke, N. J. D. A Note on a General Definition of the Coefficient of Determination. Biometrika, 78(3):691, September 1991. ISSN 00063444. doi: 10.2307/ 2337038. URL https://www.jstor.org/stable/2337038? origin=crossref. 
Niemczyk, M., Wojda, T., and Kaliszewski, A. Biomass productivity of selected poplar (Populus spp.) cultivars in short rotations in northern Poland§. New Zealand Journal of Forestry Science, 46(1), December 2016. ISSN 11795395. doi: 10.1186/s40490-016-0077-8. URL http://nzjforestryscience. springeropen.com/articles/10.1186/s40490-016-0077-8.

Nothdurft, A. Ein nichtlineares, hierarchisches und gemischtes Modell für das Baum-Höhenwachstum der Fichte (Picea abies (L.) Karst.) in BadenWürttemberg. Dissertation, Georg-August Universität Göttingen, Göttingen, February 2007. URL http://ediss.uni-goettingen.de/bitstream/handle/ 11858/00-1735-0000-0006-B0FC-1/nothdurft.pdf? sequence=1.

Oberländer, T. BwinReader Plugin (Interconnection of BWinPro and GrolMP), 2019. Unpublished.

Olsen, A. bezier: Toolkit for Bezier Curves and Splines, 2018. URL https: //CRAN.R-project.org/package=bezier. Version 1.1.2.

Oppelt, A. L., Kurth, W., and Godbold, D. L. Topology, scaling relations and Leonardo's rule in root systems from African tree species. Tree Physiology, 21(2-3):117-128, February 2001. ISSN 0829-318X, 1758-4469. doi: 10.1093/treephys/21.2-3.117. URL https://academic.oup.com/treephys/ article-lookup/doi/10.1093/treephys/21.2-3.117.

O'Reilly, O. and Tresierras, T. On the evolution of intrinsic curvature in rod-based models of growth in long slender plant stems. International Journal of Solids and Structures, 48(9):1239-1247, May 2011. ISSN 00207683. doi: 10.1016/ j.ijsolstr.2010.12.006. URL http://linkinghub.elsevier.com/retrieve/pii/ S0020768310004427.

Pellis, A., Laureysens, I., and Ceulemans, R. Genetic Variation of the Bud and Leaf Phenology of Seventeen Poplar Clones in a Short Rotation Coppice Culture. Plant Biology, 6(1):38-46, January 2004. ISSN 1435-8603, 1438-8677. doi: 10.1055/s-2003-44746. URL http://doi.wiley.com/10.1055/ s-2003-44746.

Pewsey, A., Neuhäuser, M., and Ruxton, G. D. Circular statistics in R. Oxford 
Univ. Press, Oxford, 1. ed edition, 2013. ISBN 978-0-19-165076-5 978-0-19967113-7. OCLC: 865566482.

Piepho, H. P., Buchse, A., and Emrich, K. A Hitchhiker's Guide to Mixed Models for Randomized Experiments. Journal of Agronomy and Crop Science, 189(5):310-322, October 2003. ISSN 0931-2250, 1439-037X. doi: 10.1046/j. 1439-037X.2003.00049.x. URL http://doi.wiley.com/10.1046/j.1439-037X. $2003.00049 . x$.

Pinheiro, J., Bates, D., DebRoy, S., Sarkar, D., and R Core Team. nlme: Linear and Nonlinear Mixed Effects Models, 2019. URL https://CRAN.R-project. org/package=nlme. Version 3.1-139.

Pinheiro, J. C. and Bates, D. M. Mixed-effects models in S and S-PLUS. Statistics and computing. Springer, New York, 2000. ISBN 978-0-387-98957-0.

Plazas Cebrian, A. Datenanalyse für Strukturmodelle dreier Pappelklone auf Kurzumtriebsplantagen. Bachelor thesis, Georg-August Universität Göttingen, Göttingen, October 2014.

Polhemus. 3space - FASTRAK - USER MANUAL, June 2012. URL https:// polhemus.com/_assets/img/FASTRAK_User_Manual_OPMOOPI002-G.pdf.

Pradal, C. and Cokelaer, T. MTG User Guide, December 2009. URL http:// openalea.gforge.inria.fr/doc/vplants/mtg/doc/html/user/. Last visited: 2019-03-11.

Pradal, C., Dufour-Kowalski, S., Boudon, F., Fournier, C., and Godin, C. OpenAlea: a visual programming and component-based software platform for plant modelling. Functional Plant Biology, 35(10):751, 2008. ISSN 1445-4408. doi: 10.1071/FP08084. URL http://www .publish.csiro.au/?paper=FP08084.

Pretzsch, H., Biber, P., and Ďurský, J. The single tree-based stand simulator SILVA: construction, application and evaluation. Forest Ecology and Management, 162(1):3-21, June 2002. ISSN 03781127. doi: 10.1016/ S0378-1127(02)00047-6. URL https://linkinghub.elsevier.com/retrieve/ pii/S0378112702000476. 
Pretzsch, H. Forest Dynamics, Growth and Yield. Springer Berlin Heidelberg, Berlin, Heidelberg, 2010. ISBN 978-3-540-88306-7 978-3-540-88307-4. URL http://link.springer.com/10.1007/978-3-540-88307-4.

Pretzsch, H. Grundlagen der Waldwachstumsforschung. Springer Berlin Heidelberg, Berlin, Heidelberg, 2019. ISBN 978-3-662-58154-4 978-3-662-581551. doi: 10.1007/978-3-662-58155-1. URL http://link.springer.com/10.1007/ 978-3-662-58155-1.

Prusinkiewicz, P. and Lindenmayer, A. The algorithmic beauty of plants. The virtual laboratory. Springer, New York, 2004. ISBN 0-387-94676-4.

Pyörälä, J., Liang, X., Saarinen, N., Kankare, V., Wang, Y., Holopainen, M., Hyyppä, J., and Vastaranta, M. Assessing branching structure for biomass and wood quality estimation using terrestrial laser scanning point clouds. Canadian Journal of Remote Sensing, 44(5):462-475, September 2018. ISSN 0703-8992, 1712-7971. doi: 10.1080/07038992.2018.1557040. URL https: //www.tandfonline.com/doi/full/10.1080/07038992.2018.1557040.

Quaye, A. K. and Volk, T. A. Biomass production and soil nutrients in organic and inorganic fertilized willow biomass production systems. Biomass and Bioenergy, 57:113-125, October 2013. ISSN 0961-9534. doi: 10. 1016/j.biombioe.2013.08.002. URL http://www.sciencedirect.com/science/ article/pii/S0961953413003486.

R Core Team. R: A Language and Environment for Statistical Computing, 2019. URL https://www.R-project.org/. Version 3.6.0.

Rasband, W. and Team, I. D. ImageJ, September 2017. URL https://imagej. nih.gov/ij. Version 1.51q.

Rauscher, H. M., Isebrands, J. G., Host, G. E., Dickson, R. E., Dickmann, D. I., Crow, T. R., and Michael, D. A. ECOPHYS: An ecophysiological growth process model for juvenile poplar. Tree Physiology, 7(1-2-3-4):255281, December 1990. ISSN 0829-318X, 1758-4469. doi: 10.1093/treephys/ 7.1-2-3-4.255. URL http://treephys.oxfordjournals.org/lookup/doi/10. 1093/treephys/7.1-2-3-4.255. 
Renger, M., Bohne, K., Facklam, M., Harrach, T., Riek, W., Schäfer, W., Wessolek, G., and Zacharias, G. Ergebnisse und Vorschläge der DBGArbeitsgruppe „Kennwerte des Bodengefüges“ zur Schätzung bodenphysikalischer Kennwerte. In Wessolek, G., Kaupenjohann, M., and Renger, M., editors, Bodenphysikalische Kennwerte und Berechnungsverfahren für die Praxis, volume 40 of Bodenökologie und Bodengenese, pages 4-48. Technische Universität Berlin, Berlin, 2009.

Ritz, C. and Streibig, J. C., editors. Nonlinear Regression with R. Springer New York, New York, NY, 2009. ISBN 978-0-387-09615-5 978-0-387-09616-2. URL http://link.springer.com/10.1007/978-0-387-09616-2.

Robinson, A. P. and Hamann, J. D. Forest Analytics with R. Springer New York, New York, NY, 2011. ISBN 978-1-4419-7761-8 978-1-4419-7762-5. URL http: //link.springer.com/10.1007/978-1-4419-7762-5.

Roloff, A. Baumkronen: Verständnis und praktische Bedeutung eines komplexen Naturphänomens. Ulmer, Stuttgart, 2001. ISBN 978-3-8001-3193-8.

Room, P., Maillette, L., and Hanan, J. Module and Metamer Dynamics and Virtual Plants. In Advances in Ecological Research, volume 25, pages 105-157. Elsevier, 1994. ISBN 978-0-12-013925-5. doi: 10.1016/S0065-2504(08)60214-7. URL https://linkinghub.elsevier .com/retrieve/pii/S0065250408602147.

RStudio Team. RStudio: Integrated Development Environment for R. RStudio, Inc., Boston, MA, 2016. URL http://www.rstudio.com/.

Röhle, H. Arbeitskreis Biomasse: Verfahrensempfehlungen zur Methodik der Biomasseermittlung in Kurzumtriebsbeständen. DVFFA-Sektion Ertragskunde, Jahrestagung, pages 220-226, 2009. URL http://www.nw-fva. de/ nagel/SektionErtragskunde/band2009/Tag2009_25.pdf.

Röhle, H. Standortleistungsschätzung und Biomasseermittlung in Kurzumtriebsplantagen. Allgemeine Forst- und Jagdzeitung, 184(11/12):237-246, 2013.

Sampson, D. A. and Ceulemans, R. SECRETS: simulated carbon fluxes from a mixed coniferous/deciduous Belgian forest. Forest Ecosystem Modelling. 
Upscaling and Remote Sensing. SPB Academic Publishing, The Hague, the Netherlands, pages 95-108, 1999.

Sands, P. J. 3pgpjs-a user-friendly interface to 3-PG, the Landsberg and Waring model of forest productivity. Technical Report 140, Cooperative Research Centre for Sustainable Production Forestry and CSIRO Forestry and Forest Products, Hobart, Australia, May 2004a.

Sands, P. J. Adaptation of 3-PG to novel species : guidelines for data collection and parameter assignment. Technical Report 141, Cooperative Research Centre for Sustainable Production Forestry and CSIRO Forestry and Forest Products, Hobart, Australia, May 2004b. URL http://3pg.sites.olt.ubc.ca/ files/2014/04/3-PG-guidelines.TR141.pdf.

Scheinost, W. C. 3d - Pflanzendigitalisierung: Erstellung eines dreidimensionalen Gehölzmodells unter Verwendung eines elektromagnetischen Digitalisierungsinstrumentes. Master's thesis, Universität für Bodenkultur, Wien, September 2012. URL https://www.google.com/url?sa=t\&rct= $j \& q=\& e s r c=s \&$ ource $=$ web\&cd $=4 \& c a d=r j$ a\&uact $=8 \& v e d=2$ ahUKEw $j$ Py $52 \mathrm{n}-$ TgAhUJaVAKHYexB8gQFjADegQIARAC\&url=https\%3A\%2F\%2Fzidapps. boku.ac . at\%2Fabstracts\%2Fdownload.php\%3Fdataset_id\%3D9674\%26property_id\% 3D107\&usg=AOvVaw2B-C_wL08G-San_6U3AWIK.

Schmidt, A. Der rechnerische Ausgleich von Bestandeshöhenkurven. Forstwissenschaftliches Centralblatt, 86(6):370-382, December 1967. ISSN 1612-4669, 1612-4677. doi: 10.1007/BF01821235. URL http://link. springer.com/10.1007/BF01821235.

Schmidt, M., Kiviste, A., and von Gadow, K. A spatially explicit height-diameter model for Scots pine in Estonia. European Journal of Forest Research, 130(2):303-315, March 2011. ISSN 1612-4669, 1612-4677. doi: 10.1007/s10342-010-0434-8. URL http://link.springer.com/10.1007/ s10342-010-0434-8.

Schmidt, M., Breidenbach, J., and Astrup, R. Longitudinal height-diameter curves for Norway spruce, Scots pine and silver birch in Norway based on shape 
constraint additive regression models. Forest Ecosystems, 5(1):9, December 2018. ISSN 2197-5620. doi: 10.1186/s40663-017-0125-8. URL https:// forestecosyst.springeropen.com/articles/10.1186/s40663-017-0125-8.

Schoneberg, S. Ein klimasensitives, autoregressives Modell zur Beschreibung der Einzelbaum Mortalität. Dissertation, Georg-August-Universität Göttingen, Göttingen, 2017. URL http://hdl.handle.net/11858/ 00-1735-0000-0023-3F91-0.

Schulla, J. Model Description WaSiM (Water balance Simulation Model), July 2017. URL http://www.wasim.ch/downloads/doku/wasim/wasim_2017_en. pdf.

Schön, M. Structural-functional concepts in forest modelling applicable for higher resolution of forest ecosystem simulations. Dissertation, Technická univerzita vo Zvolene, Zvolen, May 2014.

Seidel, D. and Ammer, C. Efficient measurements of basal area in short rotation forests based on terrestrial laser scanning under special consideration of shadowing. iForest - Biogeosciences and Forestry, 7(4):227-232, August 2014. ISSN 19717458. doi: 10.3832/ifor1084-007. URL http://www.sisef.it/ iforest/?doi=10.3832/if or 1084-007.

Seidel, D., Beyer, F., Hertel, D., Fleck, S., and Leuschner, C. 3d-laser scanning: A non-destructive method for studying above- ground biomass and growth of juvenile trees. Agricultural and Forest Meteorology, 151(10):1305-1311, October 2011. ISSN 01681923. doi: 10.1016/j.agrformet.2011.05.013. URL https://linkinghub.elsevier.com/retrieve/pii/S0168192311001717.

Seidel, D., Albert, K., Fehrmann, L., and Ammer, C. The potential of terrestrial laser scanning for the estimation of understory biomass in coppicewith-standard systems. Biomass and Bioenergy, 47:20-25, December 2012. ISSN 0961-9534. doi: 10.1016/j.biombioe.2012.10.009. URL http://www. sciencedirect.com/science/article/pii/S096195341200431X.

Sennerby-Forsse, L., Ferm, A., and Kauppi, A. COPPICING ABILITY AND SUSTAINABILITY. In Ecophysiology of short rotation forest crops, pages 146-172. Elsevier Applied Science, London ; New York, 1992. ISBN 1-85166-848-9. 
Sevel, L., Nord-Larsen, T., Ingerslev, M., Jørgensen, U., and RaulundRasmussen, K. Fertilization of SRC Willow, I: Biomass Production Response. BioEnergy Research, 7(1):319-328, March 2014. ISSN 1939-1234, 19391242. doi: 10.1007/s12155-013-9371-y. URL http://link.springer.com/10. 1007/s12155-013-9371-y.

Sievänen, R., Nikinmaa, E., Nygren, P., Ozier-Lafontaine, H., Perttunen, J., and Hakula, $\mathrm{H}$. Components of functional-structural tree models. Annals of Forest Science, 57(5):399-412, June 2000. ISSN 1286-4560, 1297-966X. doi: 10.1051/forest:2000131. URL http://www .edpsciences .org/10.1051/forest: 2000131.

Sievänen, R., Godin, C., DeJong, T. M., and Nikinmaa, E. Functional-structural plant models: a growing paradigm for plant studies. Annals of Botany, 114 (4):599-603, September 2014. doi: 10.1093/aob/mcu175. URL http://aob. oxfordjournals.org/content/114/4/599. abstract.

Skibbe, K. Entwicklung eines Ertragsschätzers für Kurzumtriebsbestände aus Weide. Dissertation, Technische Universität Dresden, Dresden, February 2016. URL http://nbn-resolving.de/urn:nbn:de:bsz:14-qucosa-206245.

Stewart, M. B. On Least Squares Estimation when the Dependent Variable is Grouped. The Review of Economic Studies, 50(4):737, October 1983. ISSN 00346527. doi: 10.2307/2297773. URL https://academic.oup.com/restud/ article-lookup/doi/10.2307/2297773.

Stiehm, C. and Hofmann, M. Evaluierung von Genotyp-Umwelt Interaktionen bei Pappel und Weide auf landwirtschaftlichen Standorten in kurzen Umtriebszeiten - Ergebnisse aus dem ProLoc Verbundvorhaben. Landbauforschung - applied agricultural and forestry research, 68(1/2):67-82, December 2018. doi: 10.3220/LBF1542350476000. URL http://doi.org/10.3220/ LBF1542350476000.

Stoyan, D. and Stoyan, H. Fractals, random shapes, and point fields: methods of geometrical statistics. Wiley series in probability and mathematical statistics. Wiley, Chichester ; New York, 1994. ISBN 978-0-471-93757-9. 
Surový, P. 3d in forestry. In Honorary Workshop - 110 years of prof. Václav Korf, Prag, October 2017.

Surový, P., Ribeiro, N., and Pereira, J. S. Observations on 3-dimensional crown growth of Stone pine. Agroforestry Systems, 82(2):105-110, June 2011. ISSN 0167-4366, 1572-9680. doi: 10.1007/s10457-010-9344-5. URL http://link. springer.com/10.1007/s10457-010-9344-5.

Taylor, G. Populus: Arabidopsis for Forestry. Do We Need a Model Tree? Annals of Botany, 90(6):681-689, October 2002. ISSN 0305-7364. doi: 10.1093/aob/ mcf255.

Thomas, E. Feldversuchswesen: 196 Tabellen. Number 8319 in UTB Agrarwissenschaften. Verlag Eugen Ulmer, Stuttgart, 2006. ISBN 978-3-8252-8319-3 978-3-8001-2841-9.

Van Volkenburgh, E. and Taylor, G. Leaf growth physiology. In Stettler, R. F., editor, Biology of Populus and its implications for management and conservation, number 40337 in NRC, pages 283-296. National Research Council Canada, Ottawa, 1996. ISBN 978-0-660-16506-6.

Venables, W. N. and Ripley, B. D. Modern Applied Statistics with S. Statistics and computing. Springer, New York, 4. ed., [nachdr.] edition, 2010. ISBN 9780-387-21706-2 978-1-4419-3008-8.

Verlinden, M., Broeckx, L., Van den Bulcke, J., Van Acker, J., and Ceulemans, R. Comparative study of biomass determinants of 12 poplar (Populus) genotypes in a high-density short-rotation culture. Forest Ecology and Management, 307: 101-111, November 2013. ISSN 03781127. doi: 10.1016/j.foreco.2013.06.062. URL http://linkinghub.elsevier.com/retrieve/pii/S0378112713004313.

Vos, J., Evers, J. B., Buck-Sorlin, G. H., Andrieu, B., Chelle, M., and Visser, P. H. B. Functional-structural plant modelling: a new versatile tool in crop science. Journal of Experimental Botany, 61(8):2101-2115, May 2010. ISSN 0022-0957. doi: $10.1093 / j x b / \operatorname{erp} 345$. URL http://jxb.oxfordjournals.org/ lookup/doi/10.1093/jxb/erp345. 
Warton, D. I. and Hui, F. K. C. The arcsine is asinine: the analysis of proportions in ecology. Ecology, 92(1):3-10, January 2011. ISSN 0012-9658. doi: 10.1890/ 10-0340.1. URL http://www.esajournals.org/doi/abs/10.1890/10-0340.1.

Wasilczuk, K. Implementation, Test und Dokumentation einer nutzerfreundlichen Schnittstellensoftware für den 3D-Scanner FASTRAK. Bachelor thesis, GeorgAugust Universität Göttingen, Göttingen, November 2012. URL http://www. uni-forst.gwdg.de/ ${ }^{\sim}$ wkurth/wasilczuk_ba.pdf.

Wickham, H. Tidy Data. Journal of Statistical Software, 59(10):23, August 2014. URL http://www.jstats oft.org/v59/i10.

Wickham, H. ggplot2: Elegant Graphics for Data Analysis. Use R! Springer, Cham, second edition edition, 2016. ISBN 978-3-319-24277-4. URL https: //ggplot2.tidyverse.org.

Wickham, H. tidyverse: Easily Install and Load the 'Tidyverse', 2017. URL https: //CRAN.R-project.org/package=tidyverse. Version 1.2.1.

Williams, E., Matheson, C., and Harwood, C. Experimental design and analysis for tree improvement. CSIRO, Collingwood, Vic., 2002. ISBN 0-643-06259-9.

Wissenschaftlicher Beirat Agrarpolitik beim Bundesministerium für Ernährung, Landwirtschaft und Verbraucherschutz (WBA). Nutzung von Biomasse zur Energiegewinnung - Empfehlungen an die Politik, November 2007. URL http://www.bmel.de/SharedDocs/Downloads/Ministerium/Beiraete/ Agrarpolitik/GutachtenWBA.pdf?_-blob=publicationFile.

Wittich, W. Die Standorte der Pappel. In Hesmer, H., editor, Das Pappelbuch, pages 98-110. Verlag des Deutschen Pappelvereins, Bonn, 1951.

Wittmann, O., Blume, H.-P., and Filipinski, M. SOIL CLASSIFICATION OF THE FEDERAL REPUBLIC OF GERMANY. Mitteilungen der Deutschen Bodenkundlichen Gesellschaft, 84:253-275, 1997. URL https://www.dbges.de/ de/system/files/AG_Bodensystematik/Klassifikation-engl.pdf.

Wu, R. L. Genetic mapping of QTLs affecting tree growth and architecture in Populus: implication for ideotype breeding. Theoretical and Applied Genetics, 
96(3-4):447-457, March 1998. ISSN 0040-5752, 1432-2242. doi: 10.1007/ s001220050761. URL http://link.springer.com/10.1007/s001220050761.

Wu, R. and Hinckley, T. M. Phenotypic Plasticity of Sylleptic Branching: Genetic Design of Tree Architecture. Critical Reviews in Plant Sciences, 20(5):467-485, September 2001. ISSN 0735-2689, 1549-7836. doi: 10.1080/07352689.2001. 10131827. URL https://ww.tandfonline.com/doi/full/10.1080/07352689. 2001.10131827.

Yan, H.-P. A Dynamic, Architectural Plant Model Simulating Resource-dependent Growth. Annals of Botany, 93(5):591-602, March 2004. ISSN 0305-7364, 1095-8290. doi: 10.1093/aob/mch078. URL https://academic.oup.com/aob/ article-lookup/doi/10.1093/aob/mch078.

Yoshimoto, A., Surový, P., Konoshima, M., and Kurth, W. Constructing tree stem form from digitized surface measurements by a programming approach within discrete mathematics. Trees, 28(6):1577-1588, December 2014. ISSN 0931-1890, 1432-2285. doi: 10.1007/s00468-014-1065-3. URL http://link. springer.com/10.1007/s00468-014-1065-3.

Zalesny, R. S., Donner, D. M., Coyle, D. R., and Headlee, W. L. An approach for siting poplar energy production systems to increase productivity and associated ecosystem services. Forest Ecology and Management, 284:45-58, November 2012. ISSN 03781127. doi: 10.1016/j.foreco.2012.07.022. URL http://linkinghub.elsevier.com/retrieve/pii/S0378112712004276.

Zalesny, R. S. J., Bauer, E. O., and Riemenschneider, R. Use of Belowground Growing Degree Days to Predict Rooting of Dormant Hardwood Cuttings of Populus. Silvae genetica, 53(4-5):154-160, 2004. 